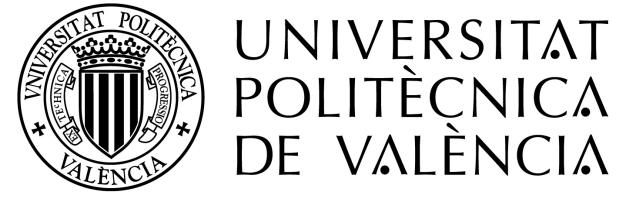

DEPARTAMENT DE MATEMÀTICA APLICADA

\title{
COMPUTATIONAL METHODS FOR RANDOM DIFFERENTIAL EQUATIONS: PROBABILITY DENSITY FUNCTION AND ESTIMATION OF THE PARAMETERS
}

\author{
AUTHOR \\ JULIA CALATAYUD GREGORI \\ ADVISER \\ PhD JUAN CARLOS CORTÉS LÓPEZ
}

València, December 2019 

To my mum and husband 

This thesis has been developed and written at the Institute of Multidisciplinary Mathematics (IMM), Universitat Politècnica de València, with the research group on Dynamical systems with uncertainty and applications.

I was awarded from Borses Ferran Sunyer i Balaguer 2019, by Fundació Ferran Sunyer i Balaguer (Institut d'Estudis Catalans), for a research stay of three months (May-July 2019) at Centre de Mathématiques Appliquées (CMAP UMR7641), École Polytechnique, Palaiseau, France.

The work has been supported by the Spanish Ministerio de Economía y Competitividad grant MTM2017-89664-P, with project Problemas Dinámicos con Incertidumbre Simulable: Modelización Matemática, Análisis, Computación y Aplicaciones. 



\section{Abstract}

Mathematical models based on deterministic differential equations do not take into account the inherent uncertainty of the physical phenomenon (in a wide sense) under study. In addition, inaccuracies in the collected data often arise due to errors in the measurements. It thus becomes necessary to treat the input parameters of the model as random quantities, in the form of random variables or stochastic processes. This gives rise to the study of random ordinary and partial differential equations.

The computation of the probability density function of the stochastic solution is important for uncertainty quantification of the model output. Although such computation is a difficult objective in general, certain stochastic expansions for the model coefficients allow faithful representations for the stochastic solution, which permits approximating its density function. In this regard, KarhunenLoève and generalized polynomial chaos expansions become powerful tools for the density approximation. Also, methods based on discretizations from finite difference numerical schemes permit approximating the stochastic solution, therefore its probability density function.

The main part of this dissertation aims at approximating the probability density function of important mathematical models with uncertainties in their formulation. Specifically, in this thesis we study, in the stochastic sense, the following models that arise in different scientific areas: in Physics, the model for the damped pendulum; in Biology and Epidemiology, the models for logistic growth and Bertalanffy, as well as epidemiological models; and in Thermodynamics, the heat partial differential equation. We rely on Karhunen-Loève and 
generalized polynomial chaos expansions and on finite difference schemes for the density approximation of the solution.

These techniques are only applicable when we have a forward model in which the input parameters have certain probability distributions already set. When the model coefficients are estimated from collected data, we have an inverse problem. The Bayesian inference approach allows estimating the probability distribution of the model parameters from their prior probability distribution and the likelihood of the data. Uncertainty quantification for the model output is then carried out using the posterior predictive distribution.

In this regard, the last part of the thesis shows the estimation of the distributions of the model parameters from experimental data on bacteria growth. To do so, a hybrid method that combines Bayesian parameter estimation and generalized polynomial chaos expansions is used.

Keywords: random ordinary and partial differential equation, uncertainty quantification, probability density function, spectral expansions, Bayesian inverse problem.

Mathematics Subject Classification 2010: 34F05, 35R60, 60H10, 60H15, $60 \mathrm{H} 35,62 \mathrm{~F} 15$. 


\section{Resum}

Els models matemàtics basats en equacions diferencials deterministes no tenen en compte la incertesa inherent al fenomen físic (en un sentit ampli) sota estudi. A més a més, sovint es produeixen inexactituds en les dades recollides a causa d'errors de mesurament. Es fa així necessari tractar els paràmetres d'entrada del model com a quantitats aleatòries, en forma de variables aleatòries o processos estocàstics. Açò dóna lloc a l'estudi de les equacions diferencials aleatòries.

El càlcul de la funció de densitat de probabilitat de la solució estocàstica és important per a quantificar la incertesa de la sortida del model. Tot i que, en general, aquest càlcul és un objectiu difícil d'assolir, certes expansions estocàstiques dels coeficients del model donen lloc a representacions fidels de la solució estocàstica, el que permet aproximar la seua funció de densitat. En aquest sentit, les expansions de Karhunen-Loève i de caos polinomial generalitzat esdevenen eines per a l'esmentada aproximació de la densitat. A més a més, els mètodes basats en discretitzacions mitjançant esquemes numèrics de diferències finites permeten aproximar la solució estocàstica, per tant la seua funció de densitat de probabilitat.

La part principal d'aquesta dissertació té com a objectiu aproximar la funció de densitat de probabilitat d'importants models matemàtics amb incerteses en la seua formulació. Concretament, en aquesta memòria s'estudien, en un sentit estocàstic, els següents models que apareixen en diferents àrees científiques: en Física, el model del pèndol amortit; en Biologia i Epidemiologia, els models de creixement logístic i de Bertalanffy, així com models de 
tipus epidemiològic; i en Termodinàmica, l'equació en derivades parcials de la calor. Per a l'aproximació de la densitat de la solució, ens basem en expansions de Karhunen-Loève i de caos polinomial generalitzat i en esquemes de diferències finites.

Aquestes tècniques només són aplicables quan tenim un model cap avant en què els paràmetres d'entrada tenen ja determinades distribucions de probabilitat. Quan els coeficients del model s'estimen a partir de les dades recollides, tenim un problema invers. L'enfocament de la inferència Bayesiana permet estimar la distribució de probabilitat dels paràmetres del model a partir de la seua distribució de probabilitat prèvia i la versemblança de les dades. La quantificació de la incertesa per a la resposta del model es fa mitjançant la distribució predictiva a posteriori.

En aquest sentit, l'última part de la tesi mostra l'estimació de les distribucions dels paràmetres del model a partir de dades experimentals sobre el creixement de bacteris. Per a fer-ho, s'utilitza un mètode híbrid que combina l'estimació de paràmetres Bayesiana i els desenvolupaments de caos polinomial generalitzat.

Paraules clau: equació diferencial aleatòria, quantificació de la incertesa, funció de densitat de probabilitat, expansions espectrals, problema invers Bayesià.

Classificació temàtica de matemàtiques 2010: 34F05, 35R60, 60H10, $60 \mathrm{H} 15,60 \mathrm{H} 35,62 \mathrm{~F} 15$. 


\section{Resumen}

Los modelos matemáticos basados en ecuaciones diferenciales deterministas no tienen en cuenta la incertidumbre inherente del fenómeno físico (en un sentido amplio) bajo estudio. Además, a menudo se producen inexactitudes en los datos recopilados debido a errores en las mediciones. Por lo tanto, es necesario tratar los parámetros de entrada del modelo como cantidades aleatorias, en forma de variables aleatorias o procesos estocásticos. Esto da lugar al estudio de las ecuaciones diferenciales aleatorias.

El cálculo de la función de densidad de probabilidad de la solución estocástica es importante en la cuantificación de la incertidumbre de la respuesta del modelo. Aunque dicho cálculo es un objetivo difícil en general, ciertas expansiones estocásticas para los coeficientes del modelo dan lugar a representaciones fieles de la solución estocástica, lo que permite aproximar su función de densidad. En este sentido, las expansiones de Karhunen-Loève y de caos polinomial generalizado constituyen herramientas para dicha aproximación de la densidad. Además, los métodos basados en discretizaciones de esquemas numéricos de diferencias finitas permiten aproximar la solución estocástica, por lo tanto, su función de densidad de probabilidad.

La parte principal de esta disertación tiene como objetivo aproximar la función de densidad de probabilidad de modelos matemáticos importantes con incertidumbre en su formulación. Concretamente, en esta memoria se estudian, en un sentido estocástico, los siguientes modelos que aparecen en diferentes áreas científicas: en Física, el modelo del péndulo amortiguado; en Biología y Epidemiología, los modelos de crecimiento logístico y de Bertalanffy, así como 
modelos de tipo epidemiológico; y en Termodinámica, la ecuación en derivadas parciales del calor. Utilizamos expansiones de Karhunen-Loève y de caos polinomial generalizado y esquemas de diferencias finitas para la aproximación de la densidad de la solución.

Estas técnicas solo son aplicables cuando tenemos un modelo directo en el que los parámetros de entrada ya tienen determinadas distribuciones de probabilidad establecidas. Cuando los coeficientes del modelo se estiman a partir de los datos recopilados, tenemos un problema inverso. El enfoque de inferencia Bayesiana permite estimar la distribución de probabilidad de los parámetros del modelo a partir de su distribución de probabilidad previa y la verosimilitud de los datos. La cuantificación de la incertidumbre para la respuesta del modelo se lleva a cabo utilizando la distribución predictiva a posteriori.

En este sentido, la última parte de la tesis muestra la estimación de las distribuciones de los parámetros del modelo a partir de datos experimentales sobre el crecimiento de bacterias. Para hacerlo, se utiliza un método híbrido que combina la estimación de parámetros Bayesianos y los desarrollos de caos polinomial generalizado.

Palabras clave: ecuación diferencial aleatoria, cuantificación de la incertidumbre, función de densidad de probabilidad, expansiones espectrales, problema inverso Bayesiano.

Clasificación temática de matemáticas 2010: 34F05, 35R60, 60H10, $60 \mathrm{H} 15,60 \mathrm{H} 35,62 \mathrm{~F} 15$. 


\section{Contents}

1 Introduction 1

2 The damped pendulum random differential equation: stochastic \begin{tabular}{|l|l|l|}
\hline analysis via the computation of the probability density function & 5
\end{tabular}

2.1 Introduction and motivation $\ldots \ldots \ldots \ldots \ldots \ldots \ldots \ldots \ldots$

2.2 Probability density function of the solution stochastic process $\ldots \ldots \ldots \ldots$

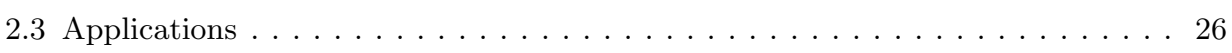

2.4 Conclusions . . . . . . . . . . . . . . . . . . . . . . . . . . . 37

3 Approximation of the density function of random non-autonomous \begin{tabular}{ll}
\hline logistic-type differential equations & 39
\end{tabular}

3.1 Introduction and motivation $\ldots \ldots \ldots \ldots \ldots \ldots \ldots \ldots \ldots \ldots \ldots$

3.2 Main results . . . . . . . . . . . . . . . . . . . . . . . 42

3.3 Numerical examples . . . . . . . . . . . . . . . . . . . . . . 47

3.4 Conclusions. . . . . . . . . . . . . . . . . . . . . . . . . . . 53 


\section{Mathematical methods for the randomized non-autonomous Berta-}

lanffy model 55

4.1 Introduction and motivation . . . . . . . . . . . . . . . . . . 56

4.2 Solving the random non-autonomous Bertalanffy model . . . . . . . . . . . . 57

4.3 Obtaining the probability density function of the solution stochastic process . . 60

4.4 Numerical examples . . . . . . . . . . . . . . . . . . . . . . . . . 70

4.5 Conclusions . . . . . . . . . . . . . . . . . . . . . . 78

5 Combining polynomial chaos expansions and the random variable transformation technique to approximate the density function of \begin{tabular}{ll}
\hline stochastic problems. & 79
\end{tabular}

5.1 Introduction . . . . . . . . . . . . . . . . . . . . . . 80

5.2 Method . . . . . . . . . . . . . . . . . . . . . . 81

5.3 Numerical Experiments. . . . . . . . . . . . . . . . . . . . . . . . 86

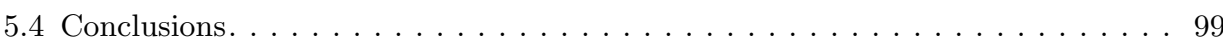

6 Uncertainty quantification for random parabolic equations via the approximation of the probability density function 101

6.1 Introduction and motivation . . . . . . . . . . . . . . . . . . 102

6.2 Preliminaries . . . . . . . . . . . . . . . . . . . . . . . . 103

6.3 Solution to the randomized heat equation with non-homogeneous Dirichlet

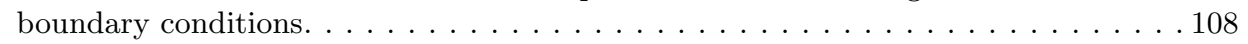

6.4 Approximation of the probability density function of the solution stochastic

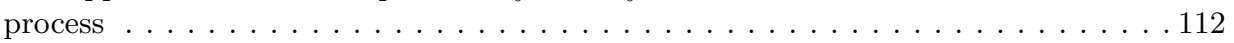

6.5 Approximation of the expectation and variance of the solution stochastic pro-

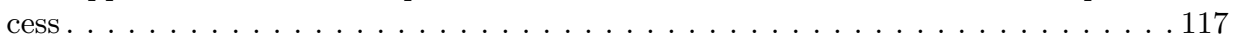

6.6 Applications . . . . . . . . . . . . . . . . . . . . . . . . . . 119

6.7 Conclusions . . . . . . . . . . . . . . . . . . . . . 131

7 Probability density function approximation to the random heat

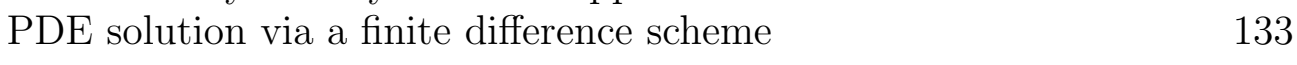

7.1 Introduction and motivation . . . . . . . . . . . . . . . . . . . . . 134

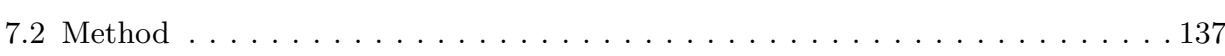


7.3 Computational aspects and algorithm . . . . . . . . . . . . . . . . 141

7.4 Numerical examples . . . . . . . . . . . . . . . . . . . . . . . . . . . . 142

7.5 Conclusions . . . . . . . . . . . . . . . . . . . . . . . . . . 148

8 Improvement of random differential models of growth of anaerobic \begin{tabular}{|lll}
\hline photosynthetic bacteria by combining Bayesian inference and gPC & 149 \\
\hline
\end{tabular}

8.1 Introduction . . . . . . . . . . . . . . . . . . . . . . . . 150

8.2 Data on anaerobic photosynthetic bacterial growth . . . . . . . . . . . . . . 152

8.3 Logistic model. . . . . . . . . . . . . . . . . . . . . . . . . . . . . . . 154

8.4 Combining Bayesian inference and gPC . . . . . . . . . . . . . . . . 158

8.5 Improvement of the logistic model . . . . . . . . . . . . . . . . . . . . . 162

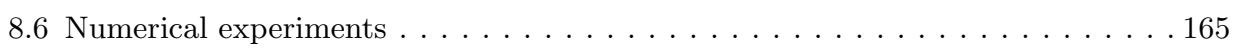

8.7 Conclusions . . . . . . . . . . . . . . . . . . . . . . . . . . . 179

9 Conclusions 181

Bibliography 183 



\section{List of Figures}

2.1 Density function $f_{X(t)}(x)$ for $t=0.1$ in Example 2.19 . . . . . 29

2.2 Density function $f_{X(t)}(x)$ for $t=0.8$ in Example 2.20 . . . . 29

2.3 Density function $f_{X(0.2)}(x)$ in Example 2.21 . . . . . . . . . 30

2.4 First: Density function $f_{X(0.1)}(x)$. Second: Density function $f_{X(0.8)}(x)$. Both in the context of Example 2.22 and interpreting the Brownian motion $Y(t)=B(t)$ as a Gaussian stochastic

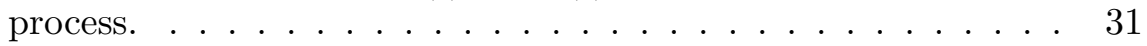

2.5 First: Density function $f_{X_{15}(0.1)}(x)$. Second: Density function $f_{X_{15}(0.8)}(x)$. Both in the context of Example 2.22 and interpreting the Brownian motion $Y(t)=B(t)$ via its Karhunen-Loève

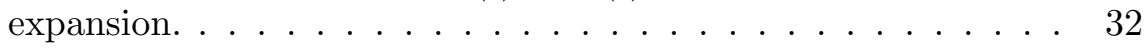

2.6 Density function $f_{X_{N}(0.5)}(x)$ for $N=1$ (up left), $N=2$ (up right), $N=3$ (center left), $N=4$ (center right), $N=5$ (down left) and $N=6$ (down right) in Example 2.23:| $\ldots \ldots \ldots .34$

2.7 Density function $f_{X_{N}(0.5)}(x)$ for $N=1$ (up left), $N=2$ (up right), $N=3$ (center left), $N=4$ (center right), $N=5$ (down left) and $N=6$ (down right) in Example $2.24 t \ldots \ldots .35$ 
2.8 Density function $f_{X_{N}(0.3)}(x)$ for $N=1$ (up left), $N=2$ (up right), $N=3$ (center left), $N=4$ (center right), $N=5$ (down left) and $N=6$ (down right) in Example 2.25. $\ldots \ldots . .36$

3.1 Plot of $f_{1}^{N}(p, t)$ for orders of truncation $N=1,2,3$ and for $t=0.2$ and $t=0.6$, in Example $3.6 .6 \ldots \ldots . . \ldots 48$

$3.2 \quad$ Plot of $f_{1}^{N}(p, t)$ for orders of truncation $N=1,2,3$ and for $t=0.2$ and $t=0.6$, in Example $3.7 .6 \ldots \ldots \ldots$

$3.3 \quad$ Plot of $f_{1}^{N}(p, t)$ for orders of truncation $N=1,2,3$ and for

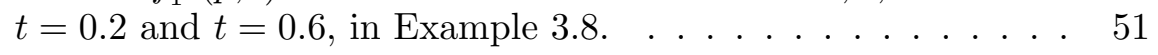

3.4 Plot of $f_{1}^{N}(p, t)$ for orders of truncation $N=1,2,3$ and for

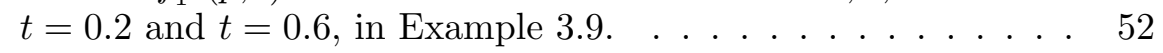

$4.1 \quad$ Density $f_{x_{N}(0.5)}(x)$ for $N=1,2,3,4,5,6$. Example 4.13 . . . . 73

4.2 Density $f_{x_{N}(0.3)}(x)$ for $N=1,2,3,4,5,6$. Example 4.14 . . . 75

\begin{tabular}{|lll|l|l|l}
\hline 4.3 & Density $f_{x_{N}(0.3)}(x)$ for $N=1,2,3,4,5,6$. Example & 4.15 \\
\hline
\end{tabular}

\begin{tabular}{ll}
5.1 & Exact graph of $f_{y(t)}(y)$ for $t=0.5$ (first) and $t=1$ (second) in \\
\hline & Example 5.2 . $\ldots \ldots \ldots \ldots \ldots$
\end{tabular}

5.2 Approximation of $f_{y(t)}(y)$ via generalized polynomial chaos (gPC) and random variable transformation (RVT) for $t=0.5$ (first plot) and $t=1$ (second plot) in Example 5.2 , Observe the rapid convergence to the exact density function $f_{y(t)}(y)$ as $p$

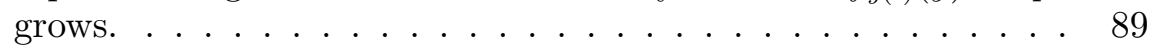

5.3 Population size of Rhodobacter capsulatus in Example 5.3 .3$. . . 91

5.4 Model for the $R$. capsulatus population with $p=4$ in Example 5.3. The circles represent the actual population size, the solid line shows the estimates via $\mathbb{E}[y(t)]$, and the dashed lines reflect the confidence interval. . . . . . . . . . . . . . 92

5.5 Approximation of $f_{y(t)}(y)$ via gPC and RVT for $t=4$ in Example 5.3 . Comparison with a Kernel density estimation. . . . . . 93 
5.6 Approximation of $f_{y(m)}(y)$ via gPC and RVT for $m=30$ in Example 5.4. Comparison with a Kernel density estimation. From $p \geq 5$, catastrophic numerical errors invalidate the results, so we are restricted to $p \leq 4.6$. . . . . . . . . . . . . . . 95

5.7 Model for the percentage of nonsmokers Spanish men aged over 16 years old during the period 1995-2014 in Example 5.5. The circles represent the actual data, the solid line shows the estimates via $\mathbb{E}[y(m)]$, and the dashed lines reflect the confidence interval. . . . . . . . . . . . . . . . 9 96

5.8 Approximation of $f_{y(m)}(y)$ via gPC and RVT for $m=15$ (first plot) and $m=18$ (second plot) in Example 5.5 . Observe the rapid convergence to the exact density function $f_{y(m)}(y)$. The results agree with a Kernel density estimation. . . . . . . . . . 97

5.9 Model for the percentage of nonsmokers Spanish men aged over 16 years old during the period 1995-2014 in Remark 5.6 of Example 5.5 , with two random input parameters. The circles represent the actual data, the solid line shows the estimates via $\mathbb{E}[y(m)]$, and the dashed lines reflect the confidence interval. . . 98

5.10 Influence of the parameters $a$ and $b$ in the nonsmokers model prediction, Remark 5.6 from Example 5.5$]$. . . . . . . . . . . . 98

6.1 Paths of the initial condition $\phi(x)$ for three different outcomes $\omega$. Example 6.16.|. . . . . . . . . . . . . . . . . 123

6.2 Approximation (6.17) for $N=1,2,3,4$ at $(x, t)=(5,0.2)$. Example 6.16.|....................... 124

6.3 Paths of the initial condition $\phi(x)$ for three different outcomes w. Example 6.17|]. . . . . . . . . . . . . . . . . . 125

6.4 Approximation (6.17) for $N=1,2,3,4$ at $(x, t)=(1,0.1)$. Example 6.17]..................... 126

6.5 Paths of the initial condition $\phi(x)$ for three different outcomes $\omega$. Example 6.18, . . . . . . . . . . . . . . . . . . . . . . 127

6.6 Approximation $(6.15)$ for $N=1,2,3,4$ at $(x, t)=(5,0.2)$. Example 6.18. . . . . . . . . . . . . . . . . . . . . . 128 
6.7 Paths of the initial condition $\phi(x)$ for three different outcomes $\omega$. Example 6.19]. . . . . . . . . . . . . . 129

6.8 Approximation $(6.15)$ for $N=1,2,3,4$ at $(x, t)=(1,0.1)$. Ex-

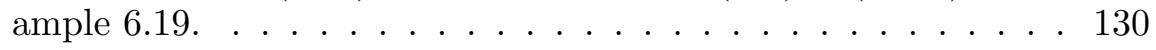

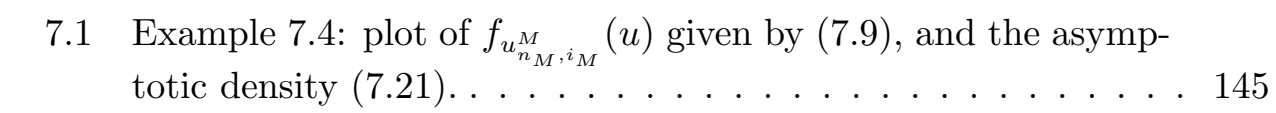

\begin{tabular}{|ll|l|l|l|l}
\hline 7.2 & Example & 7.5 & plot of $f_{u_{n_{M}, i_{M}}^{M}}(u)$ given by & 7.9 \\
\hline
\end{tabular}

8.1 Population size of $R$. capsulatus (first plot) and C. vibrioforme (second plot). . . . . . . . . . . . . . . . 153

8.2 Least squares fitting of $R$. capsulatus (first plot) and C. vibrioforme (second plot) under the logistic model. The real data is denoted by $\circ$ and the fitting is given by the black continuous line. 155

8.3 Posterior distributions for the model parameters of $R$. capsulatus under the random logistic model (8.1). . . . . . . . . . . . 167

8.4 Posterior distributions for the model parameters of C. vibrioforme under the random logistic model (8.1). . . . . . . . . . . 168

8.5 Model fitting for $R$. capsulatus (first plot) and $C$. vibrioforme (second plot) under the random logistic model (8.1). The real data is denoted by $\circ$, the fitting is given by the black continuous line and the 0.95 credible interval is drawn with dashed lines. . 168

8.6 Posterior distributions for the model parameters of $R$. capsula\begin{tabular}{ll|l} 
tus under the random logistic model (8.1) with gPC expansions. & 170
\end{tabular}

8.7 Posterior distributions for the model parameters of C. vibrioforme under the random logistic model (8.1) with gPC expansions. 170

8.8 Model fitting for $R$. capsulatus (first plot) and C. vibrioforme (second plot) under the random logistic model (8.1) with gPC expansions. The real data is denoted by $\circ$, the fitting is given by the black continuous line and the 0.95 credible interval is drawn with dashed lines. . . . . . . . . . . . . . . . . . 171 
8.9 Least squares fitting of $R$. capsulatus (first plot) and C. vibrioforme (second plot) under the new model (8.11). The real data is denoted by $\circ$ and the fitting is given by the black continuous

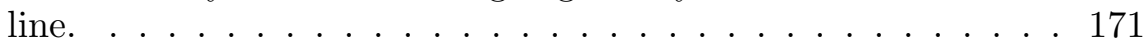

8.10 Least squares fitting of $R$. capsulatus (first plot) and C. vibrioforme (second plot) under the new model (8.12). The real data is denoted by $\circ$ and the fitting is given by the black continuous

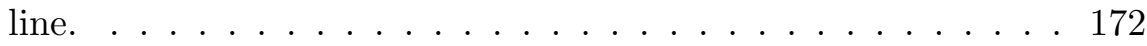

8.11 Posterior distributions for the model parameters of $R$. capsulatus under the new model (8.11) with gPC expansions. . . . . . 174

8.12 Posterior distributions for the model parameters of $C$. vibrioforme under the new model (8.11) with gPC expansions. . . . . 175

8.13 Model fitting for $R$. capsulatus (first plot) and C. vibrioforme (second plot) under the new model (8.11) with gPC expansions. The real data is denoted by $\circ$, the fitting is given by the black continuous line and the 0.95 credible interval is drawn with dashed lines. . . . . . . . . . . . . . . . . 175

8.14 Posterior distributions for the model parameters of $R$. capsulatus under the new model (8.12) with gPC expansions. . . . . . 176

8.15 Posterior distributions for the model parameters of C. vibrioforme under the new model (8.12) with gPC expansions. . . . . 177

8.16 Model fitting for $R$. capsulatus (first plot) and $C$. vibrioforme (second plot) under the new model (8.12) with gPC expansions. The real data is denoted by $\circ$, the fitting is given by the black continuous line and the 0.95 credible interval is drawn with dashed lines . . . . . . . . . . . . . . . . . 178 



\section{List of Tables}

3.1 Approximations $\mathbb{E}\left[P_{N}(t)\right]$ and $\mathbb{V}\left[P_{N}(t)\right]$ for $N=1,2,3$ and $t=$ $0.2, t=0.6$, in Example $3.6 .6 \ldots \ldots \ldots$

$3.2 \quad$ Approximations $\mathbb{E}\left[P_{N}(t)\right]$ and $\mathbb{V}\left[P_{N}(t)\right]$ for $N=1,2,3$ and $t=$ $0.2, t=0.6$, in Example|3.7. $\ldots \ldots \ldots \ldots \ldots$

3.3 Approximations $\mathbb{E}\left[P_{N}(t)\right]$ and $\mathbb{V}\left[P_{N}(t)\right]$ for $N=1,2,3$ and $t=$ $0.2, t=0.6$, in Example $3.8 . \ldots \ldots \ldots \ldots \ldots \ldots$

3.4 Approximations $\mathbb{E}\left[P_{N}(t)\right]$ and $\mathbb{V}\left[P_{N}(t)\right]$ for $N=1,2,3$ and $t=$ $0.2, t=0.6$, in Example $3.9 . \mid \ldots \ldots \ldots \ldots \ldots$

$4.1 \quad \mathbb{E}\left[x_{N}(0.5, \omega)\right]$ and $\mathbb{V}\left[x_{N}(0.5, \omega)\right]$ for $N=1,2,3,4,5,6$. Example

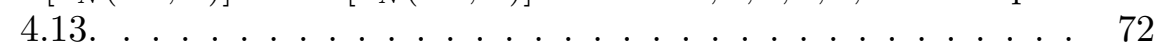

$4.2 \mathbb{E}\left[x_{N}(0.3, \omega)\right]$ and $\mathbb{V}\left[x_{N}(0.3, \omega)\right]$ for $N=1,2,3,4,5,6$. Example

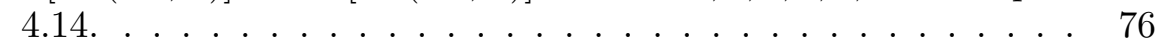

$4.3 \mathbb{E}\left[x_{N}(0.3, \omega)\right]$ and $\mathbb{V}\left[x_{N}(0.3, \omega)\right]$ for $N=1,2,3,4,5,6$. Example

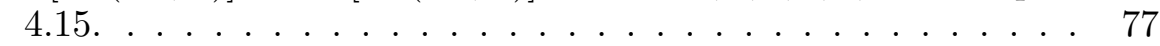

5.1 Error $\left\|f_{y(t)}-f_{\tilde{y}^{p}(t)}\right\|_{\mathrm{L}^{1}(\mathbb{R})}$ for $t=0.5$ and $t=1$, and $p=$ $1,2,3,4,5,6$, in Example 5.2 . Note the rapid convergence (exponential) to the exact density function $f_{y(t)}$ as $p$ increases.. .90 
$5.2 \quad$ Bacteria population sizes of $R$. capsulatus [127], Example [5.3] . 90

5.3 Consecutive difference $\left\|f_{\tilde{y}^{p+1}(t)}-f_{\tilde{y}^{p}(t)}\right\|_{\mathrm{L}^{1}(\mathbb{R})}$ for $t=4$, and $p=$ $1,2,3$, in Example 5.3 . Notice the rapid convergence of the consecutive differences to 0 as $p$ grows. . . . . . . . . . 93

5.4 Percentage of nonsmokers Spanish men aged over 16 years old during the period 1995-2014. Source [103]. $\ldots \ldots \ldots \ldots 9$

6.1 Difference of two consecutive orders of approximation $N$ and $N+1$ given by (6.17), for $N=1,2,3$. Example 6.16. $\ldots \ldots .123$

$6.2 \quad$ Approximations of $\mathbb{E}[u(5,0.2)]$ and $\mathbb{V}[u(5,0.2)]$ for $N=1,2,3,4$ constructed by (6.18) and (6.19), respectively, being $f_{u_{N}(x, t)}$ given by (6.17). Example $6.16 \mid \ldots \ldots \ldots \ldots \ldots \ldots$

6.3 Difference of two consecutive orders of approximation $N$ and $N+1$ given by (6.17), for $N=1,2,3$. Example $6.17 . \mid \ldots \ldots 125$

$6.4 \quad$ Approximations of $\mathbb{E}[u(1,0.1)]$ and $\mathbb{V}[u(1,0.1)]$ for $N=1,2,3,4$ constructed by (6.18) and $(6.19)$, respectively, being $f_{u_{N}(x, t)}$ given by (6.17). Example 6.17$] \ldots \ldots \ldots \ldots \ldots$

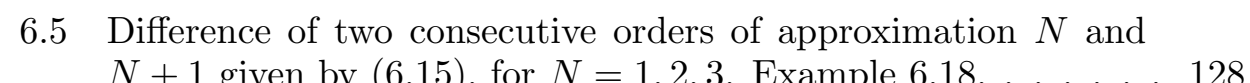

6.6 Approximations of $\mathbb{E}[u(5,0.2)]$ and $\mathbb{V}[u(5,0.2)]$ for $N=1,2,3,4$ constructed by $(6.18)$ and $(6.19)$, respectively, being $f_{u_{N}(x, t)}$ given by $(6.15)$. Example 6.18$] \ldots \ldots \ldots \ldots \ldots \ldots$

6.7 Difference of two consecutive orders of approximation $N$ and $N+1$ given by $(6.15)$, for $N=1,2,3$. Example 6.19$] \ldots \ldots 130$

6.8 Approximations of $\mathbb{E}[u(1,0.1)]$ and $\mathbb{V}[u(1,0.1)]$ for $N=1,2,3,4$ constructed by (6.18) and (6.19), respectively, being $f_{u_{N}(x, t)}$ given by $(6.15)$. Example 6.19$] \ldots \ldots \ldots \ldots \ldots$

7.1 Example 7.4 approximations for $\mathbb{E}\left[u\left(x_{0}, t_{0}\right)\right]$ and $\mathbb{V}\left[u\left(x_{0}, t_{0}\right)\right]$ obtained with (7.13). $\ldots \ldots \ldots \ldots \ldots \ldots$

7.2 Example 7.4 : approximations for $\mathbb{E}\left[u\left(x_{0}, t_{0}\right)\right]$ and $\mathbb{V}\left[u\left(x_{0}, t_{0}\right)\right]$ as in ref. [47] $($ see $(7.14)-(7.20)) . \ldots \ldots \ldots 146$ 
\begin{tabular}{|ll|l|l|l|}
\hline 7.3 & Example & 7.5 & approximations for $\mathbb{E}\left[u\left(x_{0}, t_{0}\right)\right]$ and $\mathbb{V}\left[u\left(x_{0}, t_{0}\right)\right]$ \\
\hline
\end{tabular} obtained with $(7.13) . \ldots \ldots \ldots \ldots \ldots \ldots \ldots$

7.4 Example 7.5 , approximations for $\mathbb{E}\left[u\left(x_{0}, t_{0}\right)\right]$ and $\mathbb{V}\left[u\left(x_{0}, t_{0}\right)\right]$ as in ref. [47] $($ see $(7.14)-(7.20)) . \quad \ldots \ldots \ldots \ldots 148$

$8.1 \quad$ Bacteria population sizes [127]. . . . . . . . . . . . . . 152

8.2 Parameters for $R$. capsulatus and C. vibrioforme under the logistic model. . . . . . . . . . . . 155

8.3 Descriptive table for the posterior distributions of the parameters for $R$. capsulatus and $C$. vibrioforme under the random logistic model (8.1). . . . . . . . . . . . 167

8.4 Descriptive table for the posterior distributions of the parameters for $R$. capsulatus and $C$. vibrioforme under the random logistic model (8.1) with gPC expansions. . . . . . . . . . 169

8.5 Parameters for $R$. capsulatus and $C$. vibrioforme under the new model (8.11). ............................

$8.6 \quad$ Parameters for $R$. capsulatus and $C$. vibrioforme under the new model (8.12). . . . . . . . . . . . . . 172

8.7 Descriptive table for the posterior distributions of the parameters for $R$. capsulatus and $C$. vibrioforme under the new model (8.11) with gPC expansions. . . . . . . . . . . . . . 174

8.8 Descriptive table for the posterior distributions of the parameters for R. capsulatus and C. vibrioforme under the new model $(8.12)$ with gPC expansions. . . . . . . . . . 178 



\section{Chapter 1}

\section{Introduction}

Mathematical models based on random differential and difference equations consider input parameters as random variables or stochastic processes [104, 125, 129]. The aim of this kind of models is at capturing the uncertainty often met in the analysis of complex phenomena. This randomness comes from errors in measurements or estimations, a lack of information, or ignorance of the phenomenon under analysis.

The primary goal of uncertainty quantification consists in understanding the main probabilistic features of the model output, by computing its statistical moments (mainly the mean and the variance), confidence intervals, etc. [124]. The most well-known method to approach this problem is Monte Carlo simulation [60]. It is easy to implement and effective, but may be computationally expensive. A quite latest alternative is based on generalized polynomial chaos (gPC) expansions and the stochastic Galerkin projection technique [24, 33, 35, 46, 90, 117, 126, 138, 142, 143, 144]. The stochastic solution is approximated as a linear combination of orthogonal polynomials with respect to the distributions of the input coefficients. This expansion usually converges at algebraic/exponential rate (spectral convergence) in the mean square sense [21, 121, 142].

Apart from computing the mean and the variance, a major goal in dealing with stochastic models is to calculate the set of (first) probability density functions 
of the solution process at each time instant [125, p. 35]. By means of the probability density function, all the statistical moments and confidence intervals corresponding to the solution process can be determined, provided they exist [125, Ch. 2-3]. When dealing with stochastic problems with a closedform solution, the Random Variable Transformation (RVT) technique has been widely used to calculate the density function of the solution, see [23, 29, 52] in the setting of random differential equations, [23, 53, 72, 73, 74, 146] in the context of random partial differential equations, and [44] for random difference equations. When no explicit form of the solution is available or it is given via infinite analytic expressions (such as random power series, random eigenfunctions expansions, etc.), one usually constructs a sequence of approximating processes, whose exact density functions (computed via the RVT technique) form a sequence of approximating density functions that converge rapidly. This methodology has been carried out via numerical methods [57], Karhunen-Loève expansions [17, 19] (see [89] for the theory on these expansions), polynomial expansions [14], or random power series [17, 45], and it has supposed an improvement compared with Monte Carlo simulation and Kernel density estimations in terms of rate of convergence and accuracy.

In this dissertation, we aim at approximating the probability density function of the stochastic solution to certain mathematical models with uncertainties (damped pendulum, logistic growth, Bertalanffy, heat, etc.). We will rely on spectral expansions based on Karhunen-Loève and gPC developments, and on numerical schemes based on finite differences.

These techniques are applicable when we have a forward model in which the input parameters have certain probability distributions already set. But in general, to determine the distribution of the model parameters, experimental data needs to be used. The process of adjusting the coefficients in virtue of collected data is called an inverse problem. The Bayesian approach for parameter estimation allows making statistical inference from prior probabilistic information of the parameters and the likelihood associated to the data. The output of the Bayesian inference is a posterior probability distribution for each of the parameters, which permits quantifying uncertainty via the posterior predictive distribution.

The last part of this dissertation addresses the problem of estimating the equation parameters in mathematical models on bacteria growth, using a hybrid method that combines Bayesian inference and gPC expansions.

This thesis is organized in different chapters as follows: 
In Chapters 27 the main goal is to approximate the probability density function for different random models based on ordinary and partial differential equations. We start in Chapter 2 with a linear differential equation model for the damped pendulum, where the damping ratio, the natural frequency, the initial position and velocity are random variables, and the harmonic excitation is a stochastic process. In Chapter 3 we address the nonlinear differential equation for logistic growth, where the diffusion coefficient is a stochastic process and the initial condition is a random variable. Chapter 4 studies Bertalanffy model for the growth of organisms, where the coefficients of anabolism and catabolism are considered as stochastic processes. These three chapters use Karhunen-Loève expansions for the stochastic data. In Chapter 5 we combine gPC expansions and the RVT method for the density approximation in stochastic problems, with applications to epidemic models having one random input parameter. In Chapters 6 and 7 we move to random partial differential equations: for the heat partial differential equation with uncertainties on a bounded domain, we perform density approximations using KarhunenLoève expansions for the stochastic coefficients and finite difference numerical schemes, respectively. Chapter 8 aims at adjusting model coefficients from experimental data on bacteria growth, by combining Bayesian parameter estimation and gPC expansions. The last chapter, Chapter 9, draws the main conclusions of the thesis. 

Chapter 2

\section{The damped pendulum random differential equation: stochastic analysis via the computation of the probability density function}

This chapter deals with the damped pendulum random differential equation: $\ddot{X}(t)+2 \omega_{0} \xi \dot{X}(t)+\omega_{0}^{2} X(t)=Y(t), t \in[0, T]$, with initial conditions $X(0)=X_{0}$ and $\dot{X}(0)=X_{1}$. The forcing term $Y(t)$ is a stochastic process and $X_{0}$ and $X_{1}$ are random variables in a common underlying complete probability space $(\Omega, \mathcal{F}, \mathbb{P})$. The term $X(t)$ is a stochastic process that solves the random differential equation in both the sample path and in the $\mathrm{L}^{p}$ senses. To understand the probabilistic behavior of $X(t)$, we need its joint finite-dimensional distributions. We establish mild conditions under which $X(t)$ is an absolutely continuous random variable, for each $t$, and we find its probability density function $f_{X(t)}(x)$. Thus, we obtain the first finite-dimensional distributions. In practice, we deal with two types of forcing term: $Y(t)$ is a Gaussian process, which occurs with the damped pendulum stochastic differential equation of Itô type; and $Y(t)$ can be approximated by a sequence $\left\{Y_{N}(t)\right\}_{N=1}^{\infty}$ in $\mathrm{L}^{2}([0, T] \times \Omega)$, which occurs with Karhunen-Loève expansions and some random power series. Finally, we provide numerical examples in which we choose specific random variables $X_{0}$ and $X_{1}$ and a specific stochastic process $Y(t)$, and then, we find the probability density function of $X(t)$. 


\subsection{Introduction and motivation}

The study of the damped pendulum differential equation with uncertainties has been tackled using different approaches, namely, the random and the Itô approaches, [124, pp. 96-97], 66. In the former case, uncertainty is manifested in coefficients, initial/boundary conditions and/or forcing term via random variables and/or functions whose sample behavior is regular (e.g., continuous). This approach leads to the so-called Random Differential Equations (RDEs). While in the latter case, uncertainty is forced through an irregular stochastic process such as a Wiener process or Brownian motion. In this case, Stochastic Differential Equations (SDEs) are formulated. SDEs are typically written in terms of stochastic differentials, but they are interpreted as Itô stochastic integrals. Nevertheless, if the integrand of the involved Itô integral is deterministic, SDEs can be treated as RDEs too. This is the case when the forcing term is just the White noise stochastic process. This particular situation will be studied later. Throughout our contribution RDEs will be considered only.

On the one hand, in [125, p. 161] the author analyzes the damped pendulum RDE when the forcing term is nonwhite and assuming that it is mean square continuous and wide-sense stationary with zero mean and having a given correlation function. On the other hand, recently in [71] the authors have provided an efficient computational method, based upon generalized hat basis functions, for solving stochastic Itô differential equations written in their integral equivalent form. In [71, a nice analysis of the stochastic pendulum problem is included. In both papers, a key objective is the computation of reliable approximations for the mean and the variance of the solution stochastic process. In [94, the author deals with the study of an oscillator subject to a random multiplicative noise with a spectral density (or power-spectrum) that decays as a power law at high frequencies. Although the computation of the first two statistical moments (mean and variance) of the solution is an important goal, in general, a more ambitious target is the computation of the exact or approximate probability distribution of the solution stochastic process to RDEs. In particular, a major challenge is to determine the probability density function of the solution, since from it one can obtain a full characterization of all one-dimensional statistical moments of the solution (hence including, just as particular cases, the mean and the variance). We point out that the computation of the probability density function of the solution stochastic process of some random ordinary and partial differential equations describing relevant problems in Physics and Engineering has been achieved, [53, 118, 55, 54, 72, 73, 74, 59, 146]. 
In this chapter, we deal with the computation of the probability density function of the random initial value problem

$$
\left\{\begin{array}{l}
\ddot{X}(t)+2 \omega_{0} \xi \dot{X}(t)+\omega_{0}^{2} X(t)=Y(t), t \in[0, T], \\
X(0)=X_{0}, \\
\dot{X}(0)=X_{1},
\end{array}\right.
$$

under mild conditions on the random input data $X_{0}, X_{1}$ and $Y(t)$. Here, $T>0, w_{0} \neq 0, \xi \neq 0$ and $\xi^{2}<1$ (underdamped case) are constant [125, Example 7.2]. We are assuming an underlying complete probability space $(\Omega, \mathcal{F}, \mathbb{P})$, where $\Omega$ is the sample space, $\mathcal{F}$ is a $\sigma$-algebra of events and $\mathbb{P}$ is a probability measure. The outcomes (i.e., the elements of $\Omega$ ) will be generically denoted by $\omega$. The initial position $X_{0}$ and the initial velocity $X_{1}$ are random variables and the source/forcing term $Y(t)$ is a stochastic process. The term $X(t)$ is a stochastic process that solves (2.1) in some probabilistic sense, see Theorem 2.2. When we want to make the dependence on $\omega \in \Omega$ explicit, we will write $X_{0}(\omega), X_{1}(\omega), Y(t, \omega)$ and $X(t, \omega)$.

Notation 2.1 In this chapter, we will work with Lebesgue spaces. Given a measure space $(S, \mathcal{A}, \mu)$, where $S$ is an abstract set, $\mathcal{A}$ is the $\sigma$-algebra and $\mu$ is the measure, we will use the notation $\mathrm{L}^{p}(S), 1 \leq p<\infty$, for the real-valued $p$-integrable measurable mappings in the Lebesgue sense: $f: S \rightarrow \mathbb{R}$ such that $\|f\|_{\mathrm{L}^{p}(S)}:=\left(\int_{S}|f|^{p} \mathrm{~d} \mu\right)^{1 / p}<\infty$. When $p=\infty$, the norm in $\mathrm{L}^{\infty}(S)$ is given by $\|f\|_{\mathrm{L}^{\infty}(S)}=\inf \{\sup \{|f(x)|: x \in S \backslash N\}: \mu(N)=0\}$ (this norm is the socalled "essential supremum"). In this chapter, we will work with the particular cases of $S$ being a real interval $I$ with the real Lebesgue measure $\mathrm{d} \mu=\mathrm{d} x$, $S$ being a sample space $\Omega_{0}$ with a probability measure $\mu=\mathbb{Q}$ (we write $\mathrm{d} \mu=\mathrm{d} \mathbb{Q}=\mathbb{Q}(\mathrm{d} x)$, where $x \in \Omega_{0}$ is the variable of integration), and $S$ being a product space $I \times \Omega_{0}$ with the product measure $\mathrm{d} \mu=\mathrm{d} x \times \mathrm{d} \mathbb{Q}$. Notice that, in the latter case, the $p$-norm of a measurable map $f: I \times \Omega_{0} \rightarrow \mathbb{R}$ is given by $\|f\|_{\mathrm{L}^{p}\left(I \times \Omega_{0}\right)}=\left(\mathbb{E}\left[\int_{I}|f(x)|^{p} \mathrm{~d} x\right]\right)^{1 / p}$, where $\mathbb{E}$ stands for the expectation operator with respect to the probability $\mathbb{Q}$. The important case $p=2$ corresponds to the so-called mean square stochastic convergence that will be explicitly used in this chapter. The shorten notation a.e. and a.s. will stand for "almost every" or "almost everywhere", and "almost surely", respectively.

One way to find a formal solution to 2.1 is by acting as in the deterministic case. The second-order linear differential equation is equivalent to the following first-order system of linear differential equations:

$$
\left(\begin{array}{c}
\dot{X}(t) \\
\ddot{X}(t)
\end{array}\right)=\left(\begin{array}{cc}
0 & 1 \\
-\omega_{0}^{2} & -2 \omega_{0} \xi
\end{array}\right)\left(\begin{array}{l}
X(t) \\
\dot{X}(t)
\end{array}\right)+\left(\begin{array}{c}
0 \\
Y(t)
\end{array}\right) .
$$


Using the theory on deterministic first-order systems of linear differential equations, one obtains that a formal solution to 2.1 is given by

$$
\begin{aligned}
X(t) & =\left(\frac{\xi \mathrm{e}^{-\omega_{0} \xi t} \sin \left(\omega_{1} t\right)}{\sqrt{1-\xi^{2}}}+\mathrm{e}^{-\omega_{0} \xi t} \cos \left(\omega_{1} t\right)\right) X_{0} \\
& +\frac{\mathrm{e}^{-\omega_{0} \xi t} \sin \left(\omega_{1} t\right)}{\omega_{1}} X_{1}+\int_{0}^{t} h(t-s) Y(s) \mathrm{d} s
\end{aligned}
$$

where

$$
\omega_{1}=\omega_{0} \sqrt{1-\xi^{2}} \neq 0, \quad h(t)=\frac{1}{\omega_{1}} \mathrm{e}^{-\xi \omega_{0} t} \sin \left(\omega_{1} t\right) .
$$

The goal of this contribution is to provide a comprehensive stochastic analysis of the random initial value problem (2.1) via the exact or approximate computation of the probability density function of its solution stochastic process (2.3)-2.4. Furthermore, for the sake of completeness, we first establish the following theorem where we show that the stochastic process $(2.3)-(2.4)$ is a rigorous solution to 2.1 in the two main probabilistic senses usually considered in dealing with RDEs, namely the sample path sense and the $\mathrm{L}^{p}$ sense (see for instance [125, Appendix A] and [125, Ch. 5-8], respectively).

Theorem 2.2 The following hold:

- Sample path solution: Suppose that the integral $\int_{0}^{t} h(t-s) Y(s) \mathrm{d} s$ in (2.3) is interpreted as a Lebesgue integral for each $\omega \in \Omega$ fixed (this is sometimes referred to as SP integral, see [129, Def. $A-1])$. If $Y$ has sample paths in $\mathrm{L}^{1}([0, T])$, then $X$ is the unique process with $C^{1}([0, T])$ sample paths, having absolutely continuous derivatives, that solve (2.1) for a.e. $t \in[0, T]$. If $Y$ has continuous sample paths, then $X$ is the unique process with $C^{2}([0, T])$ sample paths that solve (2.1) for every $t \in[0, T]$.

- L ${ }^{p}$-solution: Suppose that the integral $\int_{0}^{t} h(t-s) Y(s) \mathrm{d} s$ in $\sqrt{2.3}$ is interpreted as an $\mathrm{L}^{p}$-Riemann integral (see [125, p. 100]). If $Y$ is $\mathrm{L}^{p}$ continuous and $X_{0}, X_{1} \in \mathrm{L}^{p}(\Omega)$, then $X$ is the unique $\mathrm{L}^{p}$-solution [125, p. 118$]$.

Proof. The first part is a consequence of the theory on deterministic differential equations (see Carathéodory theory in [68, pp. 28-30]). The second part follows from [125, Th. 7.1.1]. 
We will address the following important cases regarding the random nature of the forcing term $Y(t)$ : (1) it is Gaussian, and in particular, a White noise stochastic process; (2) it can be represented via a Karhunen-Loève expansion, and (3) it can be represented via a mean square random power series.

This chapter is organized as follows. In Section 2.2, we first establish a key lemma that will be extensively used throughout the chapter. This result allows us to give useful expressions for the probability density function, $f_{X(t)}(x)$, of the solution stochastic process $X(t)$ given by $2.3-\sqrt{2.4}$ in terms of the expectation operator or explicitly. In Subsection 2.2.1, these expressions are also obtained in the significant case that the forcing term $Y(t)$ is Gaussian. This particular analysis includes the case in which $Y(t)$ is the White noise stochastic process that involves an Itô type SDE. Subsection 2.2.2 addresses the study of the RDE (2.1) in the case that the forcing term can be approximated by a stochastic process in $\mathrm{L}^{2}([0, T] \times \Omega)$. After providing general results in this setting, the analysis is divided into two important cases: (i) $Y(t)$ is expressed via a Karhunen-Loève expansion (see Subsubsection 2.2.2) and (ii) $Y(t)$ is expressed via a mean square random power series (see Subsubsection 2.2.2). Section 2.3 is devoted to illustrating our findings by means of a wide range of numerical examples. Finally, conclusions are drawn in Section 2.4.

\subsection{Probability density function of the solution stochastic process}

Our main goal in this chapter is to establish conditions under which the solution stochastic process $X(t)$ given by $2.3-(2.4)$ is an absolutely continuous random variable for each $t \in[0, T]$, and then to compute its probability density function, $f_{X(t)}(x)$. Physically, the existence and computation of the probability density function of $X(t)$ means that the probability for the response to lie in a certain set $A$ at time $t$ can be calculated as $\mathbb{P}(X(t) \in A)=\int_{A} f_{X(t)}(x) \mathrm{d} x$. This allows computing the main statistical properties of the response process $X(t)$, say the mean, variance, or any specific moment.

For that purpose, we need the following key lemma.

Lemma 2.3 [8, pp. 266-267] Let $Z_{1}$ and $Z_{2}$ be two independent real random variables defined in a common complete probability space $(\Omega, \mathcal{F}, \mathbb{P})$. Suppose that $Z_{1}$ is absolutely continuous. Then $Z_{1}+Z_{2}$ is absolutely continuous and has density function $f_{Z_{1}+Z_{2}}(u)=\mathbb{E}\left[f_{Z_{1}}\left(u-Z_{2}\right)\right]$. 
In what follows, we will impose conditions on the initial position $X_{0}$ and the initial velocity $X_{1}$, see $\boldsymbol{H}_{\mathbf{0}}$ and $\boldsymbol{H}_{\mathbf{1}}$, so that $X(t)$ is an absolutely continuous random variable, for each $t$. As we shall see in the subsequent development, Lemma 2.3 will play a key role to obtain the probability density function of $X(t), f_{X(t)}(x)$.

Fix $t \in[0, T]$. We will assume one of the following two hypotheses:

$\boldsymbol{H}_{\mathbf{0}}: X_{0}$ is absolutely continuous, $\frac{\xi \sin \left(\omega_{1} t\right)}{\sqrt{1-\xi^{2}}}+\cos \left(\omega_{1} t\right) \neq 0, X_{0}$ and $\left(X_{1}, Y\right)$ are independent 1 .

$\boldsymbol{H}_{\mathbf{1}}: X_{1}$ is absolutely continuous, $\sin \left(\omega_{1} t\right) \neq 0, X_{1}$ and $\left(X_{0}, Y\right)$ are independent.

Assume $\boldsymbol{H}_{\mathbf{0}}$. Then, using the Random Variable Transformation technique 89 , Lemma 4.12],

$$
Z_{1}=\left(\frac{\xi \mathrm{e}^{-\omega_{0} \xi t} \sin \left(\omega_{1} t\right)}{\sqrt{1-\xi^{2}}}+\mathrm{e}^{-\omega_{0} \xi t} \cos \left(\omega_{1} t\right)\right) X_{0}
$$

is absolutely continuous, with density function given by

$$
f_{Z_{1}}(z)=f_{X_{0}}\left(\frac{z}{\frac{\xi \mathrm{e}^{-\omega_{0} \xi t} \sin \left(\omega_{1} t\right)}{\sqrt{1-\xi^{2}}}+\mathrm{e}^{-\omega_{0} \xi t} \cos \left(\omega_{1} t\right)}\right) \frac{1}{\left|\frac{\xi \mathrm{e}^{-\omega_{0} \xi t} \sin \left(\omega_{1} t\right)}{\sqrt{1-\xi^{2}}}+\mathrm{e}^{-\omega_{0} \xi t} \cos \left(\omega_{1} t\right)\right|} .
$$

Taking into account expression 2.3 and applying Lemma 2.3 with $Z_{2}=$ $\frac{\mathrm{e}^{-\omega_{0} \xi t} \sin \left(\omega_{1} t\right)}{\omega_{1}} X_{1}+\int_{0}^{t} h(t-s) Y(s) \mathrm{d} s, X(t)$ is absolutely continuous and

$$
\begin{aligned}
& f_{X(t)}(x) \\
= & \mathbb{E}\left[f_{X_{0}}\left(\frac{x-\frac{\mathrm{e}^{-\omega_{0} \xi t} \sin \left(\omega_{1} t\right)}{\omega_{1}} X_{1}-\int_{0}^{t} h(t-s) Y(s) \mathrm{d} s}{\frac{\mathrm{e}^{-\omega_{0} \xi t} \sin \left(\omega_{1} t\right)}{\sqrt{1-\xi^{2}}}+\mathrm{e}^{-\omega_{0} \xi t} \cos \left(\omega_{1} t\right)}\right)\right] \frac{1}{\left|\frac{\xi \mathrm{e}^{-\omega_{0} \xi t} \sin \left(\omega_{1} t\right)}{\sqrt{1-\xi^{2}}}+\mathrm{e}^{-\omega_{0} \xi t} \cos \left(\omega_{1} t\right)\right|} .
\end{aligned}
$$

Assume $\boldsymbol{H}_{\mathbf{1}}$. Proceeding as before, but now applying Lemma 2.3 with $Z_{1}=$ $\frac{\mathrm{e}^{-\omega_{0} \xi t} \sin \left(\omega_{1} t\right)}{\omega_{1}} X_{1}$ and $Z_{2}=\left(\frac{\xi \mathrm{e}^{-\omega_{0} \xi t} \sin \left(\omega_{1} t\right)}{\sqrt{1-\xi^{2}}}+\mathrm{e}^{-\omega_{0} \xi t} \cos \left(\omega_{1} t\right)\right) X_{0}+\int_{0}^{t} h(t-s) Y(s) \mathrm{d} s$,

\footnotetext{
${ }^{1} X_{0}$ and $\left(X_{1}, Y\right)$ independent means that, for any points $t_{1}, \ldots, t_{m} \in[0, T], m \geq 1$, the random variable $X_{0}$ and the random vector $\left(X_{1}, Y\left(t_{1}\right), \ldots, Y\left(t_{m}\right)\right)$ are independent.
} 
$X(t)$ is absolutely continuous with density function

$$
\begin{aligned}
& f_{X(t)}(x) \\
= & \mathbb{E}\left[f_{X_{1}}\left(\frac{\left.x-\left(\frac{\xi \mathrm{e}^{-\omega_{0} \xi t} \sin \left(\omega_{1} t\right)}{\sqrt{1-\xi^{2}}}+\mathrm{e}^{-\omega_{0} \xi t} \cos \left(\omega_{1} t\right)\right) X_{0}-\int_{0}^{t} h(t-s) Y(s) \mathrm{d} s\right)}{\frac{\mathrm{e}^{-\omega_{0} \xi t} \sin \left(\omega_{1} t\right)}{\omega_{1}}}\right)\right] \\
& \cdot \frac{1}{\frac{\mathrm{e}^{-\omega_{0} \xi t}\left|\sin \left(\omega_{1} t\right)\right|}{\left|\omega_{1}\right|}} .
\end{aligned}
$$

Summarizing, the following result has been established:

Proposition 2.4 The following statements hold:

- Assume $\boldsymbol{H}_{\mathbf{0}}$, then the probability density function of the solution stochastic process (2.3)-2.4 to the random initial value problem (2.1) is given by 2.5.

- Assume $\boldsymbol{H}_{\mathbf{1}}$, then the probability density function of the solution stochastic process 2.3 -2.4 to the random initial value problem 2.1 is given by 2.6.

In practice, the problem is to know the probability law of $I(t)=\int_{0}^{t} h(t-$ $s) Y(s) \mathrm{d} s$. If we know the law $\mathbb{P}_{I(t)}=\mathbb{P} \circ I(t)^{-1}$ of $I(t)$, then 2.5 becomes

$$
\begin{aligned}
f_{X(t)}(x)= & \frac{1}{\left|\frac{\xi \mathrm{e}^{-\omega_{0} \xi t} \sin \left(\omega_{1} t\right)}{\sqrt{1-\xi^{2}}}+\mathrm{e}^{-\omega_{0} \xi t} \cos \left(\omega_{1} t\right)\right|} \\
& \cdot \int_{\mathbb{R}^{2}} f_{X_{0}}\left(\frac{x-\frac{\mathrm{e}^{-\omega_{0} \xi t} \sin \left(\omega_{1} t\right)}{\omega_{1}} x_{1}-y}{\frac{\xi \mathrm{e}^{-\omega_{0} \xi t} \sin \left(\omega_{1} t\right)}{\sqrt{1-\xi^{2}}}+\mathrm{e}^{-\omega_{0} \xi t} \cos \left(\omega_{1} t\right)}\right) \mathbb{P}_{\left(X_{1}, I(t)\right)}\left(\mathrm{d} x_{1}, \mathrm{~d} y\right),
\end{aligned}
$$

and (2.6) becomes

$$
\begin{aligned}
& f_{X(t)}(x) \\
= & \frac{1}{\frac{\mathrm{e}^{-\omega_{0} \xi t}\left|\sin \left(\omega_{1} t\right)\right|}{\left|\omega_{1}\right|}} \int_{\mathbb{R}^{2}} f_{X_{1}}\left(\frac{x-\left(\frac{\xi \mathrm{e}^{-\omega_{0} \xi t} \sin \left(\omega_{1} t\right)}{\sqrt{1-\xi^{2}}}+\mathrm{e}^{-\omega_{0} \xi t} \cos \left(\omega_{1} t\right)\right) x_{0}-y}{\frac{\mathrm{e}^{-\omega_{0} \xi t} \sin \left(\omega_{1} t\right)}{\omega_{1}}}\right) \\
& \cdot \mathbb{P}_{\left(X_{0}, I(t)\right)}\left(\mathrm{d} x_{0}, \mathrm{~d} y\right) .
\end{aligned}
$$


As a consequence, both expressions for the probability density function $f_{X(t)}(x)$ can be computed. In fact, one does not need to compute those integrals analytically, since by sampling from $X_{0}, X_{1}$ and $I(t)$, we can approximate the expectations in (2.5) and (2.6) as accurate as desired by the Law of Large Numbers (Monte Carlo simulation).

Our objective in the following subsections is to analyze important cases in which (2.5) and (2.6) can be computed, or at least approximated, in practice. More specifically, the subsequent analysis will be split into two cases, depending on the random nature of $Y(t)$. First, in Subsection 2.2.1, we will deal with $Y(t)$ being a Gaussian stochastic process. This case will include the damped pendulum stochastic differential equation of Itô type in Subsubsection 2.2.1. Second, in Subsection 2.2.2, we will deal with a forcing term $Y(t)$ that can be approximated in $\mathrm{L}^{2}([0, T] \times \Omega)$. This second case will include two significant situations: $Y(t)$ is expressed as a Karhunen-Loève expansion, in Subsubsection 2.2.2, and $Y(t)$ is expressed as a random power series, in Subsubsection 2.2.2,

\subsubsection{Gaussian forcing term}

Suppose that $Y(t)$ is a Gaussian stochastic process. Then the process $I(t)=$ $\left\{\int_{0}^{t} h(t-s) Y(s) \mathrm{d} s: t \in[0, T]\right\}$ is Gaussian. Indeed, if the integral is interpreted as a Lebesgue integral for each fixed $\omega \in \Omega$, then this fact follows from [16. Lemma 2.3]; if the integral is an $\mathrm{L}^{p}$-Riemann integral, $p \geq 2$, then the Gaussianity follows from [125, Th. 4.6.4]. Moreover, its mean and variance are given by

$$
\mathbb{E}[I(t)]=\int_{0}^{t} h(t-s) \mathbb{E}[Y(s)] \mathrm{d} s=: \mu_{I}(t)
$$

and

$$
\mathbb{V}[I(t)]=\int_{0}^{t} \int_{0}^{t} h\left(t-s_{1}\right) h\left(t-s_{2}\right) \operatorname{Cov}\left[Y\left(s_{1}\right), Y\left(s_{2}\right)\right] \mathrm{d} s_{1} \mathrm{~d} s_{2}=: \sigma_{I}(t)^{2},
$$

respectively. Thus, $I(t) \sim \operatorname{Normal}\left(\mu_{I}(t), \sigma_{I}(t)^{2}\right)$. From this, if $\boldsymbol{H}_{\mathbf{0}}$ holds and, moreover, the random variable $X_{1}$ and the stochastic process $Y$ are independent (which implies the independence of $X_{1}$ and $I(t)$, i.e., $\mathbb{P}_{\left(X_{1}, I(t)\right)}=$ 
$\left.\mathbb{P}_{X_{1}} \times \mathbb{P}_{I(t)}\right)$, we can compute 2.5 as

$$
\begin{aligned}
& f_{X(t)}(x)=\frac{1}{\left|\frac{\xi \mathrm{e}^{-\omega_{0} \xi t} \sin \left(\omega_{1} t\right)}{\sqrt{1-\xi^{2}}}+\mathrm{e}^{-\omega_{0} \xi t} \cos \left(\omega_{1} t\right)\right|} \\
& \quad \int_{\mathbb{R}} \int_{\mathbb{R}} f_{X_{0}}\left(\frac{x-\frac{\mathrm{e}^{-\omega_{0} \xi t} \sin \left(\omega_{1} t\right)}{\omega_{1}} x_{1}-y}{\frac{\xi \mathrm{e}^{-\omega_{0} \xi t} \sin \left(\omega_{1} t\right)}{\sqrt{1-\xi^{2}}}+\mathrm{e}^{-\omega_{0} \xi t} \cos \left(\omega_{1} t\right)}\right) f_{\operatorname{Normal}\left(\mu_{I}(t), \sigma_{I}(t)^{2}\right)}(y) \mathrm{d} y \mathbb{P}_{X_{1}}\left(\mathrm{~d} x_{1}\right) .
\end{aligned}
$$

If $\boldsymbol{H}_{\mathbf{1}}$ holds and, moreover, the random variable $X_{0}$ and the stochastic process $Y$ are independent, 2.6 is written as

$$
\begin{aligned}
& f_{X(t)}(x) \\
= & \frac{1}{\frac{\mathrm{e}^{-\omega_{0} \xi t}\left|\sin \left(\omega_{1} t\right)\right|}{\left|\omega_{1}\right|}} \int_{\mathbb{R}} \int_{\mathbb{R}} f_{X_{1}}\left(\frac{x-\left(\frac{\xi \mathrm{e}^{-\omega_{0} \xi t} \sin \left(\omega_{1} t\right)}{\sqrt{1-\xi^{2}}}+\mathrm{e}^{-\omega_{0} \xi t} \cos \left(\omega_{1} t\right)\right) x_{0}-y}{\frac{\mathrm{e}^{-\omega_{0} \xi t} \sin \left(\omega_{1} t\right)}{\omega_{1}}}\right) \\
& \cdot f_{\operatorname{Normal}\left(\mu_{I}(t), \sigma_{I}(t)^{2}\right)}(y) \mathrm{d} y \mathbb{P}_{X_{0}}\left(\mathrm{~d} x_{0}\right) .
\end{aligned}
$$

On the other hand, bearing in mind expressions 2.3 2.4 and Lemma 2.3, if $I(t) \sim \operatorname{Normal}\left(\mu_{I}(t), \sigma_{I}(t)^{2}\right)$ and $Y$ is independent of $\left(\bar{X}_{0}, X_{1}\right)$, but $\boldsymbol{H}_{\mathbf{0}}$ and $\boldsymbol{H}_{\mathbf{1}}$ do not hold, then $X(t)$ remains being absolutely continuous. By Lemma 2.3 with $Z_{1}=I(t)$ and $Z_{2}=\left(\frac{\xi \mathrm{e}^{-\omega_{0} \xi t} \sin \left(\omega_{1} t\right)}{\sqrt{1-\xi^{2}}}+\mathrm{e}^{-\omega_{0} \xi t} \cos \left(\omega_{1} t\right)\right) X_{0}+\frac{\mathrm{e}^{-\omega_{0} \xi t} \sin \left(\omega_{1} t\right)}{\omega_{1}} X_{1}$, the probability density function of $X(t)$ can be expressed as

$$
\begin{aligned}
& f_{X(t)}(x) \\
= & \mathbb{E}\left[f_{\operatorname{Normal}\left(\mu_{I}(t), \sigma_{I}(t)^{2}\right)}\left(x-\left(\frac{\xi \mathrm{e}^{-\omega_{0} \xi t} \sin \left(\omega_{1} t\right)}{\sqrt{1-\xi^{2}}}+\mathrm{e}^{-\omega_{0} \xi t} \cos \left(\omega_{1} t\right)\right) X_{0}-\frac{\mathrm{e}^{-\omega_{0} \xi t} \sin \left(\omega_{1} t\right)}{\omega_{1}} X_{1}\right)\right] \\
= & \int_{\mathbb{R}^{2}} f_{\operatorname{Normal}\left(\mu_{I}(t), \sigma_{I}(t)^{2}\right)}\left(x-\left(\frac{\xi \mathrm{e}^{-\omega_{0} \xi t} \sin \left(\omega_{1} t\right)}{\sqrt{1-\xi^{2}}}+\mathrm{e}^{-\omega_{0} \xi t} \cos \left(\omega_{1} t\right)\right) x_{0}-\frac{\mathrm{e}^{-\omega_{0} \xi t} \sin \left(\omega_{1} t\right)}{\omega_{1}} x_{1}\right) \\
& \cdot \mathbb{P}_{\left(X_{0}, X_{1}\right)}\left(\mathrm{d} x_{0}, \mathrm{~d} x_{1}\right) .
\end{aligned}
$$

Observe that, in order to derive expression (2.11), we have not needed that $X_{0}$ and $X_{1}$ be absolutely continuous random variables, respectively. This fact is a consequence of the key Lemma 2.3 .

Below, we summarize the results previously established in the important case that the forcing term $Y=Y(t)$ is a Gaussian stochastic process: 
Proposition 2.5 Let us consider the random initial value problem (2.1), where $Y=Y(t)$ is a Gaussian stochastic process. The following statements hold:

- Assume $\boldsymbol{H}_{\mathbf{0}}$ and that $X_{1}$ and $Y$ are independent, then the probability density function of the solution stochastic process (2.3)-(2.4) is given by (2.9).

- Assume $\boldsymbol{H}_{\mathbf{1}}$ and that $X_{0}$ and $Y$ are independent, then the probability density function of the solution stochastic process (2.3)-(2.4) is given by 2.10.

- Assume that $Y$ and $\left(X_{0}, X_{1}\right)$ are independent, then the probability density function of the solution stochastic process 2.3 -2.4 is given by 2.11.

Damped pendulum stochastic differential equation of Itô type

Let the forcing term $Y(t)$ be a White noise process: $Y$ is a Gaussian process, $\mathbb{E}[Y(t)]=0$ and $\mathbb{E}[Y(t) Y(s)]=\delta_{0}(t-s)$. In this case, using formulas 2.7 and 2.8), $\mu_{I}(t)=0$ and $\sigma_{I}(t)^{2}=\int_{0}^{t} h(s)^{2} \mathrm{~d} s$, therefore $I(t) \sim$ $\operatorname{Normal}\left(0, \int_{0}^{t} h(s)^{2} \mathrm{~d} s\right)$. Thus, if $Y$ is independent of $\left(X_{0}, X_{1}\right)$ and $\left(X_{0}, X_{1}\right)$ has any probability distribution, then the density function of the solution stochastic process $X(t)$ is given by (2.11). For the sake of completeness, we state this result in the following corollary:

Corollary 2.6 Let us consider the random initial value problem (2.1), where $Y=Y(t)$ is a White noise stochastic process. Assume that $Y$ and $\left(X_{0}, X_{1}\right)$ are independent. Then, the probability density function of the solution stochastic process (2.3) -2.4 is given by 2.11).

Notice that, if $Y(t)$ is a White noise process, then the random differential equation problem (2.1) becomes a stochastic differential equation of Itô type: if we denote

$$
Z(t)=\left(\begin{array}{c}
X(t) \\
\dot{X}(t)
\end{array}\right), \quad Z_{0}=\left(\begin{array}{l}
X_{0} \\
X_{1}
\end{array}\right), \quad A=\left(\begin{array}{cc}
0 & 1 \\
-\omega_{0}^{2} & -2 \omega_{0} \xi
\end{array}\right), \quad b=\left(\begin{array}{l}
0 \\
1
\end{array}\right),
$$

and $Y(t)=\dot{B}(t)$, where $B$ is a standard Brownian motion [89, Def. 5.11], then, from $(2.2)$, we obtain the following stochastic differential equation of Itô type:

$$
\left\{\begin{array}{l}
\mathrm{d} Z(t)=A Z(t) \mathrm{d} t+b \mathrm{~d} B(t), t \in[0, T], \\
Z(0)=Z_{0} .
\end{array}\right.
$$


The solution stochastic process $X(t)$ of 2.1 corresponds to the first component of $Z(t)$ :

$$
X(t)=\left(\frac{\xi \mathrm{e}^{-\omega_{0} \xi t} \sin \left(\omega_{1} t\right)}{\sqrt{1-\xi^{2}}}+\mathrm{e}^{-\omega_{0} \xi t} \cos \left(\omega_{1} t\right)\right) X_{0}+\frac{\mathrm{e}^{-\omega_{0} \xi t} \sin \left(\omega_{1} t\right)}{\omega_{1}} X_{1}+\int_{0}^{t} h(t-s) \mathrm{d} B(s),
$$

where the integral is understood in the Itô sense.

\subsubsection{Forcing term that can be approximated in $\mathrm{L}^{2}([0, T] \times \Omega)$}

In this subsection, we assume that $Y \in \mathrm{L}^{2}([0, T] \times \Omega)$ and that there exists a sequence $\left\{Y_{N}\right\}_{N=1}^{\infty}$ of stochastic processes in $\mathrm{L}^{2}([0, T] \times \Omega)$ that converges to $Y$ in the topology of $\mathrm{L}^{2}([0, T] \times \Omega)$. This occurs, as we will see, with KarhunenLoève expansions and some random power series. The first case will be studied in Subsubsection 2.2.2, whereas the second case will be analyzed in Subsubsection 2.2.2. Before considering these two important cases, in this subsection we will establish general results in order to determine computable expressions for the probability density function of the solution stochastic process (2.3)-(2.4) to the random initial value problem (2.1) (see Theorem 2.7 and Theorem 2.9 later).

From the truncation $Y_{N}$ of the forcing term $Y$, we consider a truncation of the solution stochastic process 2.3 :

$X_{N}(t)=\left(\frac{\xi \mathrm{e}^{-\omega_{0} \xi t} \sin \left(\omega_{1} t\right)}{\sqrt{1-\xi^{2}}}+\mathrm{e}^{-\omega_{0} \xi t} \cos \left(\omega_{1} t\right)\right) X_{0}+\frac{\mathrm{e}^{-\omega_{0} \xi t} \sin \left(\omega_{1} t\right)}{\omega_{1}} X_{1}+\int_{0}^{t} h(t-s) Y_{N}(s) \mathrm{d} s$,

where the integral is understood in the Lebesgue sense. Notice that $X_{N}(t) \rightarrow$ $X(t)$ as $N \rightarrow \infty$ in $\mathrm{L}^{2}(\Omega)$, for each $t \in[0, T]$. Indeed, since $Y_{N} \rightarrow Y$ as $N \rightarrow \infty$ in $\mathrm{L}^{2}([0, T] \times \Omega)$ and $h$ is bounded on $[0, T]$ (see definition of $h$ in $(2.4)$ ), we have that $h(t-s) Y_{N}(s) \rightarrow h(t-s) Y(s)$ as $N \rightarrow \infty$ in $\mathrm{L}^{2}([0, t] \times \Omega, \mathrm{d} s \times \mathrm{d} \mathbb{P})$, for each $t \in[0, T]$, so by Cauchy-Schwarz inequality,

$$
\begin{aligned}
\mathbb{E}\left[\left|X_{N}(t)-X(t)\right|^{2}\right] & =\mathbb{E}\left[\left|\int_{0}^{t} h(t-s)\left(Y_{N}(s)-Y(s)\right) \mathrm{d} s\right|^{2}\right] \\
& \leq T\|h\|_{\mathrm{L}^{\infty}([0, T])}^{2}\left\|Y_{N}-Y\right\|_{\mathrm{L}^{2}([0, T] \times \Omega)}^{2} \stackrel{N \rightarrow \infty}{\longrightarrow} 0 .
\end{aligned}
$$

This shows that $X_{N}(t) \rightarrow X(t)$ as $N \rightarrow \infty$ in $\mathrm{L}^{2}(\Omega)$, for each $t \in[0, T]$. This is important, as the main statistical information of $X(t)$, say the expectation and variance, can be approximated by using the following key properties of mean square convergence: $\lim _{N \rightarrow \infty} \mathbb{E}\left[X_{N}(t)\right]=\mathbb{E}[X(t)]$ and $\lim _{N \rightarrow \infty} \mathbb{V}\left[X_{N}(t)\right]=$ $\mathbb{V}[X(t)]$ (see [125, Th. 4.2.1, Th. 4.3.1]). 
We will assume that $\boldsymbol{H}_{\mathbf{0}}$ entails that $X_{0}$ is independent of $\left(X_{1}, Y_{1}, \ldots, Y_{N}\right)$ for all $N \geq 1$ (analogously, $\boldsymbol{H}_{\mathbf{1}}$ implies that $X_{1}$ is independent of $\left(X_{0}, Y_{1}, \ldots, Y_{N}\right)$ for all $N \geq 1$ ). Later on, in the particular cases of Karhunen-Loève expansions and random power series, we will prove this assumption in Remark 2.10 and Remark 2.14, respectively.

Under $\boldsymbol{H}_{\mathbf{0}}, X_{N}(t)$ is absolutely continuous with density function given by

$$
\begin{aligned}
f_{X_{N}(t)}(x)= & \mathbb{E}\left[f_{X_{0}}\left(\frac{x-\frac{\mathrm{e}^{-\omega_{0} \xi t} \sin \left(\omega_{1} t\right)}{\omega_{1}} X_{1}-\int_{0}^{t} h(t-s) Y_{N}(s) \mathrm{d} s}{\frac{\xi \mathrm{e}^{-\omega_{0} \xi t} \sin \left(\omega_{1} t\right)}{\sqrt{1-\xi^{2}}}+\mathrm{e}^{-\omega_{0} \xi t} \cos \left(\omega_{1} t\right)}\right)\right] \\
& \cdot \frac{1}{\left|\frac{\xi \mathrm{e}^{-\omega_{0} \xi t} \sin \left(\omega_{1} t\right)}{\sqrt{1-\xi^{2}}}+\mathrm{e}^{-\omega_{0} \xi t} \cos \left(\omega_{1} t\right)\right|}
\end{aligned}
$$

Under $\boldsymbol{H}_{\mathbf{1}}, X_{N}(t)$ is absolutely continuous with density function given by

$$
\begin{aligned}
& f_{X_{N}(t)}(x) \\
= & \mathbb{E}\left[f_{X_{1}}\left(\frac{x-\left(\frac{\xi \mathrm{e}^{-\omega_{0} \xi t} \sin \left(\omega_{1} t\right)}{\sqrt{1-\xi^{2}}}+\mathrm{e}^{-\omega_{0} \xi t} \cos \left(\omega_{1} t\right)\right) X_{0}-\int_{0}^{t} h(t-s) Y_{N}(s) \mathrm{d} s}{\frac{\mathrm{e}^{-\omega_{0} \xi t} \sin \left(\omega_{1} t\right)}{\omega_{1}}}\right)\right] \\
& \cdot \frac{1}{\frac{\mathrm{e}^{-\omega_{0} \xi t}\left|\sin \left(\omega_{1} t\right)\right|}{\left|\omega_{1}\right|}} .
\end{aligned}
$$

Theorem 2.7 The following statements for the probability density function, $f_{X(t)}(x)$, of the solution stochastic process (2.3)-2.4 to the random initial value problem (2.1) hold:

- Assume $\boldsymbol{H}_{\mathbf{0}}$. If $f_{X_{0}}$ is continuous on $\mathbb{R}$ and $f_{X_{0}}(x) \leq a+b x^{2}$, for certain $a, b>0$, then $\lim _{N \rightarrow \infty} f_{X_{N}(t)}(x)=f_{X(t)}(x)$ for all $x \in \mathbb{R}$, being $f_{X_{N}(t)}(x)$ and $f_{X(t)}(x)$ defined by (2.14) and (2.5), respectively.

- Assume $\boldsymbol{H}_{\mathbf{1}}$. If $f_{X_{1}}$ is continuous on $\mathbb{R}$ and $f_{X_{1}}(x) \leq a+b x^{2}$, for certain $a, b>0$, then $\lim _{N \rightarrow \infty} f_{X_{N}(t)}(x)=f_{X(t)}(x)$ for all $x \in \mathbb{R}$, being $f_{X_{N}(t)}(x)$ and $f_{X(t)}(x)$ defined by (2.15) and (2.6), respectively.

Proof. We will prove the first part, as the second one is analogous. Thus, let us assume $\boldsymbol{H}_{\mathbf{0}}, f_{X_{0}}$ continuous on $\mathbb{R}$ and $f_{X_{0}}(x) \leq a+b x^{2}$. 
Let

and

$$
U_{N}=\frac{x-\frac{\mathrm{e}^{-\omega_{0} \xi t} \sin \left(\omega_{1} t\right)}{\omega_{1}} X_{1}-\int_{0}^{t} h(t-s) Y_{N}(s) \mathrm{d} s}{\frac{\xi \mathrm{e}^{-\omega_{0} \xi t} \sin \left(\omega_{1} t\right)}{\sqrt{1-\xi^{2}}}+\mathrm{e}^{-\omega_{0} \xi t} \cos \left(\omega_{1} t\right)}
$$

$$
U=\frac{x-\frac{\mathrm{e}^{-\omega_{0} \xi t} \sin \left(\omega_{1} t\right)}{\omega_{1}} X_{1}-\int_{0}^{t} h(t-s) Y(s) \mathrm{d} s}{\frac{\xi \mathrm{e}^{-\omega_{0} \xi t} \sin \left(\omega_{1} t\right)}{\sqrt{1-\xi^{2}}}+\mathrm{e}^{-\omega_{0} \xi t} \cos \left(\omega_{1} t\right)} .
$$

Since $Y_{N} \rightarrow Y$ as $N \rightarrow \infty$ in $\mathrm{L}^{2}([0, T] \times \Omega)$, and by 2.13$)$, we derive that $U_{N} \rightarrow U$ as $N \rightarrow \infty$ in $\mathrm{L}^{2}(\Omega)$.

By (2.14), to conclude that $\lim _{N \rightarrow \infty} f_{X_{N}(t)}(x)=f_{X(t)}(x)$, it suffices to show $f_{X_{0}}\left(U_{N}\right) \rightarrow f_{X_{0}}(U)$ as $N \rightarrow \infty$ in $\mathrm{L}^{1}(\Omega)$ (because convergence in $\mathrm{L}^{1}(\Omega)$ implies convergence of expectations). This will follow if we show that, for every subsequence $\left\{U_{N_{k}}\right\}_{k=1}^{\infty}$ of $\left\{U_{N}\right\}_{N=1}^{\infty}$, there exists another subsequence $\left\{U_{N_{k_{l}}}\right\}_{l=1}^{\infty}$ such that $f_{X_{0}}\left(U_{N_{k_{l}}}\right) \rightarrow f_{X_{0}}(U)$ as $l \rightarrow \infty$ in $\mathrm{L}^{1}(\Omega)^{2}$. Thus, fix a subsequence $\left\{U_{N_{k}}\right\}_{k=1}^{\infty}$. Since $U_{N_{k}} \rightarrow U$ as $k \rightarrow \infty$ in $\mathrm{L}^{2}(\Omega)$, by [11, Th. 4.9] there exists a subsequence $\left\{U_{N_{k_{l}}}\right\}_{l=1}^{\infty}$ and a random variable $V \in \mathrm{L}^{2}(\Omega)$ such that $U_{N_{k_{l}}}(\omega) \rightarrow U(\omega)$ as $l \rightarrow \infty$ a.s. and $\left|U_{N_{k_{l}}}(\omega)\right| \leq V(\omega)$ a.s., for all $l \geq 1$. As $f_{X_{0}}$ is continuous on $\mathbb{R}, f_{X_{0}}\left(U_{N_{k_{l}}}(\omega)\right) \rightarrow f_{X_{0}}(U(\omega))$ as $l \rightarrow \infty$ a.s. Now, $f_{X_{0}}\left(U_{N_{k_{l}}}(\omega)\right) \leq a+b U_{N_{k_{l}}}(\omega)^{2} \leq a+b V(\omega)^{2} \in \mathrm{L}^{1}(\Omega)$. By the Dominated Convergence Theorem [113, result 11.32, p. 321], $f_{X_{0}}\left(U_{N_{k_{l}}}\right) \rightarrow f_{X_{0}}(U)$ as $l \rightarrow \infty$ in $\mathrm{L}^{1}(\Omega)$, as wanted.

Remark 2.8 The conditions $f_{X_{i}}, i=0,1$, continuous on $\mathbb{R}$ and $f_{X_{i}}(x) \leq$ $a+b x^{2}$, are the usual hypotheses imposed so that the Nemytskii operator $V \mapsto$ $f_{X_{i}}(V)$, is continuous from $\mathrm{L}^{2}(\Omega)$ to $\mathrm{L}^{1}(\Omega)$, [1, pp. 15-17], [134, pp. 154-163]. Essentially, these hypotheses are mathematical restrictions on the probabilistic features of the initial position and/or initial velocity that permit proving that the density functions of the truncations $X_{N}(t)$ tend to the density function of $X(t)$ as $N \rightarrow \infty$.

From a practical standpoint, most of the probability density functions, like Beta (with shape parameters $\alpha, \beta \geq 1$ ), Gaussian, Gamma (with shape parameter $\alpha \geq 1$ and rate parameter $\beta>0$ ), etc., are bounded, so it is enough to

\footnotetext{
${ }^{2}$ Let $\left\{a_{n}\right\}_{n=1}^{\infty}$ be a sequence and $a$ be an element in a topological space. If for every subsequence $\left\{a_{n_{k}}\right\}_{k=1}^{\infty}$, there exists a subsequence $\left\{a_{n_{k_{l}}}\right\}_{l=1}^{\infty}$ such that $a_{n_{k_{l}}} \rightarrow a$ as $l \rightarrow \infty$, then $a_{n} \rightarrow a$ as $n \rightarrow \infty$. Indeed, if $a_{n}$ does not tend to $a$, there exists a neighbourhood $A$ of $a$ and a subsequence $\left\{a_{n_{k}}\right\}_{k=1}^{\infty}$ such that $a_{n_{k}} \notin A$, for all $k \geq 1$. By hypothesis, there is a subsequence $\left\{a_{n_{k_{l}}}\right\}_{l=1}^{\infty}$ that converges to $a$. But this contradicts the fact $a_{n_{k_{l}}} \notin A$, for each $l \geq 1$.
} 
take the constant $a>0$ big enough in order that the condition $f_{X_{i}}(x) \leq a+b x^{2}$, for $i=0$ or $i=1$, holds. Nevertheless, in order to further enlarge the class of random variables for which our theoretical findings can be applied, below we generalize Theorem 2.7 by assuming that the probability density functions are almost everywhere continuous rather than continuous. Recall that a real function is said to be almost everywhere continuous if it is continuous except on a set of Lebesgue measure zero. For example, the uniform, exponential, truncated Gaussian, etc. distributions possess almost everywhere continuous density functions.

In Section 2.3, the application of both Theorem 2.7 and Theorem 2.9 will be illustrated.

Theorem 2.9 The following statements for the probability density function, $f_{X(t)}(x)$, of the solution stochastic process (2.3)-2.4 to the random initial value problem (2.1) hold:

- Assume $\boldsymbol{H}_{\mathbf{0}}$. Suppose that $f_{X_{0}}$ is a.e. continuous on $\mathbb{R}$ and $f_{X_{0}}(x) \leq$ $a+b x^{2}$ for a.e. $x \in \mathbb{R}$, for certain $a, b>0$. If, in addition, $\boldsymbol{H}_{\mathbf{1}}$ holds, then $\lim _{N \rightarrow \infty} f_{X_{N}(t)}(x)=f_{X(t)}(x)$ for all $x \in \mathbb{R}$, being $f_{X_{N}(t)}(x)$ and $f_{X}(x)$ defined by (2.14) and (2.5), respectively.

- Assume $\boldsymbol{H}_{\mathbf{1}}$. Suppose that $f_{X_{1}}$ is a.e. continuous on $\mathbb{R}$ and $f_{X_{1}}(x) \leq$ $a+b x^{2}$ for a.e. $x \in \mathbb{R}$, for certain $a, b>0$. If, in addition, $\boldsymbol{H}_{\mathbf{0}}$ holds, then $\lim _{N \rightarrow \infty} f_{X_{N}(t)}(x)=f_{X(t)}(x)$ for all $x \in \mathbb{R}$, being $f_{X_{N}(t)}(x)$ and $f_{X}(x)$ defined by (2.15) and (2.6), respectively.

Proof. We show the first part, as the second one is analogous. As in the proof of Theorem 2.7, consider $U_{N}$ and $U$ defined by (2.16) and (2.17), respectively. Recall that $U_{N} \rightarrow U$ as $N \rightarrow \infty$ in $\mathrm{L}^{2}(\Omega)$. Since $\boldsymbol{H}_{\mathbf{1}}$ holds, by Lemma $2.3 U$ is absolutely continuous. This, together with the fact that $f_{X_{0}}$ is a.e. continuous, implies that the probability that $U$ belongs to the discontinuity set of $f_{X_{0}}$ is 0. Thereby, by the Continuous Mapping Theorem [135, p. 7, Th. 2.3], $f_{X_{0}}\left(U_{N_{k_{l}}}(\omega)\right) \rightarrow f_{X_{0}}(U(\omega))$ a.s., as $l \rightarrow \infty$. Therefore, the proof from Theorem 2.7 works. 
Forcing term expressed as a Karhunen-Loève expansion

Let $Y \in \mathrm{L}^{2}([0, T] \times \Omega)$. Consider its covariance integral operator

$$
\mathcal{C}: \mathrm{L}^{2}([0, T]) \rightarrow \mathrm{L}^{2}([0, T]), \quad \mathcal{C} f(t)=\int_{0}^{T} \operatorname{Cov}[Y(t), Y(s)] f(s) \mathrm{d} s .
$$

This operator is linear, compact, self-adjoint and nonnegative-definite 89, Th. 1.68, Lemma 1.72, Lemma 1.77]. Let $J \in \mathbb{N} \cup\{\infty\}$ be the dimension of the image of $\mathcal{C}$. By Hilbert-Schmidt Theorem [89, Th. 1.73], the nonzero eigenvalues of $\mathcal{C}$, repeated according to their multiplicity, form a sequence $\left\{\nu_{j}\right\}_{j=1}^{J}$ (we will not assume any particular ordering of the sequence of nonzero eigenvalues). Moreover, the sequence $\left\{\phi_{j}\right\}_{j=1}^{\infty}$ of eigenfunctions of $\mathcal{C}$ is an orthonormal basis of $\mathrm{L}^{2}([0, T])$. Here, $\phi_{j}$ is associated to $\nu_{j}$, and if $j>J$, then $\phi_{j}$ is associated to 0 .

By Karhunen-Loève Theorem [89, Th. 5.28], the process $Y=\{Y(t): t \in$ $[0, T]\}$ can be expressed as

$$
Y(t)=\mu_{Y}(t)+\sum_{j=1}^{J} \sqrt{\nu_{j}} \phi_{j}(t) \xi_{j}
$$

where $\mu_{Y}(t)=\mathbb{E}[Y(t)]$ and $\left\{\xi_{j}\right\}_{j=1}^{J}$ is a sequence of random variables with zero expectation, unit variance and pairwise uncorrelated. These random variables $\left\{\xi_{j}\right\}_{j=1}^{J}$ have a closed expression:

$$
\xi_{j}=\frac{1}{\sqrt{\nu_{j}}} \int_{0}^{T}\left(Y(s)-\mu_{Y}(s)\right) \phi_{j}(s) \mathrm{d} s, \quad j=1, \ldots, J .
$$

Moreover, if $Y=\{Y(t): t \in[0, T]\}$ is a Gaussian process, then $\left\{\xi_{j}\right\}_{j=1}^{J}$ are independent and Gaussian. If $J=\infty$, the series $(2.19)$ converges in $\mathrm{L}^{2}([0, T] \times$ $\Omega)$.

Notice that

$$
\begin{aligned}
I(t) & =\int_{0}^{t} h(t-s) Y(s) \mathrm{d} s \\
& =\int_{0}^{t} h(t-s) \mu_{Y}(s) \mathrm{d} s+\sum_{j=1}^{J} \sqrt{\nu_{j}}\left(\int_{0}^{t} h(t-s) \phi_{j}(s) \mathrm{d} s\right) \xi_{j},
\end{aligned}
$$

where, if $J=\infty$, the series is understood in $\mathrm{L}^{2}([0, T] \times \Omega)$. 
Remark 2.10 If $\boldsymbol{H}_{\mathbf{0}}$ holds, then the random inputs $X_{0}$ and $Y$ are independent, i.e., $X_{0}$ and $\left(Y\left(t_{1}\right), \ldots, Y\left(t_{m}\right)\right)$ are independent, for each $t_{1}, \ldots, t_{m} \in$ $[0, T], m \geq 1$. By 2.20$), X_{0}$ and $\left(\xi_{1}, \ldots, \xi_{J}\right)$ are independent. Indeed, as it is proved in [16, Lemma 2.3],

$$
\xi_{j} \in{\overline{\left\{\frac{1}{\sqrt{\nu_{j}}} \sum_{k=1}^{m} \lambda_{k}\left(Y\left(t_{k}\right)-\mu_{Y}\left(t_{k}\right)\right) \phi_{j}\left(t_{k}\right): \lambda_{k} \in \mathbb{R}, t_{k} \in[0,1], m \geq 1\right\}}}^{\mathrm{L}^{2}(\Omega)}
$$

(the overline stands for the closure). Since $X_{0}$ is independent to each sum

$$
\frac{1}{\sqrt{\nu_{j}}} \sum_{k=1}^{m} \lambda_{k}\left(Y\left(t_{k}\right)-\mu_{Y}\left(t_{k}\right)\right) \phi_{j}\left(t_{k}\right)
$$

we derive that $\left(\xi_{1}, \ldots, \xi_{J}\right)$ is a limit in $\mathrm{L}^{2}\left(\Omega ; \mathbb{R}^{J}\right)$ of random vectors that are independent to $X_{0}$, which implies the independence of $X_{0}$ and $\left(\xi_{1}, \ldots, \xi_{J}\right)$.

An analogous result is satisfied if $\boldsymbol{H}_{\mathbf{1}}$ holds, instead of $\boldsymbol{H}_{\mathbf{0}}$. This concludes the remark.

Suppose that $J<\infty$. Assume that $\boldsymbol{H}_{\mathbf{0}}$ holds, that we know the probability law of the random vector $\left(\xi_{1}, \ldots, \xi_{J}\right)$ and that $X_{1}$ and $\left(\xi_{1}, \ldots, \xi_{J}\right)$ are independent. Then (2.5) becomes

$$
\begin{aligned}
& f_{X(t)}(x)=\frac{1}{\left|\frac{\xi \mathrm{e}^{-\omega_{0} \xi t} \sin \left(\omega_{1} t\right)}{\sqrt{1-\xi^{2}}}+\mathrm{e}^{-\omega_{0} \xi t} \cos \left(\omega_{1} t\right)\right|} \\
& \cdot \int_{\mathbb{R}^{J}} \int_{\mathbb{R}} f_{X_{0}}\left(\frac{x-\frac{\mathrm{e}^{-\omega_{0} \xi t} \sin \left(\omega_{1} t\right)}{\omega_{1}} x_{1}-\int_{0}^{t} h(t-s) \mu_{Y}(s) \mathrm{d} s-\sum_{j=1}^{J} \sqrt{\nu_{j}}\left(\int_{0}^{t} h(t-s) \phi_{j}(s) \mathrm{d} s\right) \xi_{j}}{\frac{\xi \mathrm{e}^{-\omega_{0} \xi t} \sin \left(\omega_{1} t\right)}{\sqrt{1-\xi^{2}}}+\mathrm{e}^{-\omega_{0} \xi t} \cos \left(\omega_{1} t\right)}\right) \\
& \cdot \mathbb{P}_{X_{1}}\left(\mathrm{~d} x_{1}\right) \mathbb{P}_{\left(\xi_{1}, \ldots, \xi_{J}\right)}\left(\mathrm{d} \xi_{1}, \ldots, \mathrm{d} \xi_{J}\right) .
\end{aligned}
$$

If $\boldsymbol{H}_{\mathbf{1}}$ holds, and $X_{0}$ and $\left(\xi_{1}, \ldots, \xi_{J}\right)$ are independent, then 2.6) becomes

$$
\begin{aligned}
& f_{X(t)}(x)=\frac{1}{\frac{\mathrm{e}^{-\omega_{0} \xi t}\left|\sin \left(\omega_{1} t\right)\right|}{\left|\omega_{1}\right|}} \\
& \cdot \int_{\mathbb{R}^{J+1}} f_{X_{1}}\left(\frac{x-\left(\frac{\xi^{-\omega_{0} \xi t} \sin \left(\omega_{1} t\right)}{\sqrt{1-\xi^{2}}}+\mathrm{e}^{-\omega_{0} \xi t} \cos \left(\omega_{1} t\right)\right) x_{0}-\int_{0}^{t} h(t-s) \mu_{Y}(s) \mathrm{d} s-\sum_{j=1}^{J} \sqrt{\nu_{j}}\left(\int_{0}^{t} h(t-s) \phi_{j}(s) \mathrm{d} s\right) \xi_{j}}{\frac{\mathrm{e}^{-\omega_{0} \xi t} \sin \left(\omega_{1} t\right)}{\omega_{1}}}\right) \\
& \cdot \mathbb{P}_{X_{0}}\left(\mathrm{~d} x_{0}\right) \mathbb{P}_{\left(\xi_{1}, \ldots, \xi_{J}\right)}\left(\mathrm{d} \xi_{1}, \ldots, \mathrm{d} \xi_{J}\right) .
\end{aligned}
$$

Therefore, both densities are computable in practice.

If $J=\infty$, for each $N \geq 1$ we may define the truncations

$$
Y_{N}(t)=\mu_{Y}(t)+\sum_{j=1}^{N} \sqrt{\nu_{j}} \phi_{j}(t) \xi_{j}
$$


Thus, we have a sequence $\left\{Y_{N}\right\}_{N=1}^{\infty}$ that converges to $Y$ in $\mathrm{L}^{2}([0, T] \times \Omega)$, according to Karhunen-Loève Theorem [89, Th. 5.28]. By what we have just seen, the density function of the truncation $X_{N}$ given by 2.12 is computable, as in 2.22 and 2.23 by taking $J=N$. If the assumptions of Theorem 2.7 or Theorem 2.9 hold, then we can approximate $f_{X(t)}$ by using $\lim _{N \rightarrow \infty} f_{X_{N}(t)}(x)=$ $f_{X(t)}(x)$.

In fact, a more general result than Theorem 2.9 can be established in this setting:

Theorem 2.11 The following statements for the probability density function, $f_{X(t)}(x)$, of the solution stochastic process (2.3)-2.4 to the random initial value problem 2.1) hold:

- Assume $\boldsymbol{H}_{\mathbf{0}}$. Suppose that $f_{X_{0}}$ is a.e. continuous on $\mathbb{R}$ and $f_{X_{0}}(x) \leq a+$ b $x^{2}$ for a.e. $x \in \mathbb{R}$, for certain $a, b>0$. If, in addition, $\boldsymbol{H}_{\mathbf{1}}$ holds or some $\xi_{j}$ is absolutely continuous, independent of $\left(X_{1}, \xi_{1}, \ldots, \xi_{j-1}, \xi_{j+1}, \ldots\right)$ and with $\int_{0}^{t} h(t-s) \phi_{j}(s) \mathrm{d} s \neq 0$, then $\lim _{N \rightarrow \infty} f_{X_{N}(t)}(x)=f_{X(t)}(x)$ for all $x \in \mathbb{R}$, being $f_{X_{N}(t)}(x)$ and $f_{X}(x)$ defined by (2.14) and (2.5), respectively.

- Assume $\boldsymbol{H}_{\mathbf{1}}$. Suppose that $f_{X_{1}}$ is a.e. continuous on $\mathbb{R}$ and $f_{X_{1}}(x) \leq a+$ b $x^{2}$ for a.e. $x \in \mathbb{R}$, for certain $a, b>0$. If, in addition, $\boldsymbol{H}_{\mathbf{0}}$ holds or some $\xi_{j}$ is absolutely continuous, independent of $\left(X_{0}, \xi_{1}, \ldots, \xi_{j-1}, \xi_{j+1}, \ldots\right)$ and with $\int_{0}^{t} h(t-s) \phi_{j}(s) \mathrm{d} s \neq 0$, then $\lim _{N \rightarrow \infty} f_{X_{N}(t)}(x)=f_{X(t)}(x)$ for all $x \in \mathbb{R}$, being $f_{X_{N}(t)}(x)$ and $f_{X}(x)$ defined by 2.15 and $(2.6)$, respectively.

Proof. We prove the first part, as the second one is analogous. As in the proof of Theorem 2.7, consider $U_{N}$ and $U$ defined by (2.16) and (2.17), respectively, where $Y_{N}$ is defined by (2.24). If some $\xi_{j}$ is absolutely continuous, independent of $\left(X_{1}, \xi_{1}, \ldots, \xi_{j-1}, \xi_{j+1}, \ldots\right)$ and with $\int_{0}^{t} h(t-s) \phi_{j}(s) \mathrm{d} s \neq 0$, by Lemma 2.3 $U$ is absolutely continuous. Thereby, the proof of Theorem 2.9 is applicable.

To provide a full analysis, it is interesting to observe that, in this setting of Karhunen-Loève expansions, sometimes $\boldsymbol{H}_{\mathbf{0}}$ and $\boldsymbol{H}_{\mathbf{1}}$ do not need to be satisfied (so both $X_{0}$ and $X_{1}$ may not be absolutely continuous). Consider the hypothesis

$H_{2}: \xi_{1}$ is absolutely continuous, $\int_{0}^{t} h(t-s) \phi_{1}(s) \mathrm{d} s \neq 0, \xi_{1}$ and the vector $\left(X_{0}, X_{1}, \xi_{2}, \ldots, \xi_{j}\right)$ are independent, for each $\left.j, 2 \leq j \leq J\right]^{3}$ (if $\xi_{1}$ does

\footnotetext{
${ }^{3}$ If $J<\infty$, this independence is reduced to $\xi_{1}$ and $\left(X_{0}, X_{1}, \xi_{2}, \ldots, \xi_{J}\right)$ be independent.
} 
not satisfy $\boldsymbol{H}_{\mathbf{2}}$ but another $\xi_{j}$ instead, $j \neq 1$, then we may reorder the eigenvalues and eigenfunctions so that $\xi_{1}$ becomes $\xi_{j}$ ).

The random variable

$$
Z_{1}=\sqrt{\nu_{1}}\left(\int_{0}^{t} h(t-s) \phi_{1}(s) \mathrm{d} s\right) \xi_{1}
$$

is absolutely continuous, with density function

$$
f_{Z_{1}}(z)=f_{\xi_{1}}\left(\frac{z}{\sqrt{\nu_{1}}\left(\int_{0}^{t} h(t-s) \phi_{1}(s) \mathrm{d} s\right)}\right) \frac{1}{\sqrt{\nu_{1}}\left|\int_{0}^{t} h(t-s) \phi_{1}(s) \mathrm{d} s\right|},
$$

as a consequence of the Random Variable Transformation technique. By (2.3), (2.21) and Lemma 2.3 , the solution $X(t)$ is absolutely continuous, with density function given by

$$
\begin{aligned}
& f_{X(t)}(x)=\frac{1}{\sqrt{\nu_{1}}\left|\int_{0}^{t} h(t-s) \phi_{1}(s) \mathrm{d} s\right|} \\
& \cdot \mathbb{E}\left[f _ { \xi _ { 1 } } \left(\frac{z-\left(\frac{\mathrm{\xi}^{-\omega_{0} \xi t} \sin \left(\omega_{1} t\right)}{\sqrt{1-\xi^{2}}}+\mathrm{e}^{-\omega_{0} \xi t} \cos \left(\omega_{1} t\right)\right) X_{0}-\frac{\mathrm{e}^{-\omega_{0} \xi t} \sin \left(\omega_{1} t\right)}{\omega_{1}} X_{1}}{\sqrt{\nu_{1}}\left(\int_{0}^{t} h(t-s) \phi_{1}(s) \mathrm{d} s\right)}\right.\right. \\
& \left.\left.+\frac{-\int_{0}^{t} h(t-s) \mu_{Y}(s) \mathrm{d} s-\sum_{j=2}^{J} \sqrt{\nu_{j}}\left(\int_{0}^{t} h(t-s) \phi_{j}(s) \mathrm{d} s\right) \xi_{j}}{\sqrt{\nu_{1}}\left(\int_{0}^{t} h(t-s) \phi_{1}(s) \mathrm{d} s\right)}\right)\right] .
\end{aligned}
$$

If $J<\infty$, we may compute this explicitly. Otherwise, if $J=\infty$, we need to consider the truncation $X_{N}(t)$ given by (2.12), where $Y_{N}$ is defined in (2.24). With analogous proofs to those of Theorem 2.7 and Theorem 2.9, one arrives at the following results:

Theorem 2.12 Assume $\boldsymbol{H}_{\mathbf{2}}$. If $f_{\xi_{1}}$ is continuous on $\mathbb{R}$ and $f_{\xi_{1}}(x) \leq a+b x^{2}$, for certain $a, b>0$, then $\lim _{N \rightarrow \infty} f_{X_{N}(t)}(x)=f_{X(t)}(x)$ for all $x \in \mathbb{R}$, where $f_{X_{N}(t)}$ is given by 2.25) with $J=N$ and $f_{X(t)}(x)$ is given by 2.25) with $J=\infty$.

Theorem 2.13 Assume $\boldsymbol{H}_{2}$. Suppose that $f_{\xi_{1}}$ is a.e. continuous on $\mathbb{R}$ and $f_{\xi_{1}}(x) \leq a+b x^{2}$ for a.e. $x \in \mathbb{R}$, for certain $a, b>0$. If, in addition, $\boldsymbol{H}_{\mathbf{0}}$ holds or $\boldsymbol{H}_{\mathbf{1}}$ holds or some other $\xi_{j}(j \neq 1)$ satisfies $\boldsymbol{H}_{\mathbf{2}}$, then $\lim _{N \rightarrow \infty} f_{X_{N}(t)}(x)=$ $f_{X(t)}(x)$ for all $x \in \mathbb{R}$, where $f_{X_{N}(t)}$ is given by (2.25) with $J=N$ and $f_{X(t)}(x)$ is given by (2.25) with $J=\infty$. 
Forcing term expressed as a random power series

Sometimes, one may work with a forcing term $Y(t)$ that is an analytic stochastic process in the mean square sense [125, p. 99]: $Y(t)=\sum_{n=0}^{\infty} Y_{n} t^{n}$, where the sum converges in $\mathrm{L}^{2}(\Omega)$, for each $t \in[0, T]$. We will assume, in addition, that the convergence of the series holds in $\mathrm{L}^{2}([0, T] \times \Omega)$. Thus, if we define the partial sums $\left\{Y_{N}(t)=\sum_{n=0}^{N} Y_{n} t^{n}\right\}_{N=0}^{\infty}$, then they converge to $Y$ in $\mathrm{L}^{2}([0, T] \times \Omega)$.

Remark 2.14 If $\boldsymbol{H}_{\mathbf{0}}$ holds, then $X_{0}$ and $Y$ are independent. Let us see that $X_{0}$ and $\left(Y_{0}, \ldots, Y_{N}\right)$ are independent, for $0 \leq N<\infty$.

We have $Y_{n}=Y^{(n)}(0) / n$ !, where $Y^{(n)}$ is the $n$-th mean square derivative of $Y(t)$ [125, Ch. 4]. Thus, if we prove that $X_{0}$ and

$$
\left(Y\left(t_{1}^{0}\right), \ldots, Y\left(t_{m_{0}}^{0}\right), Y^{\prime}\left(t_{1}^{1}\right), \ldots, Y^{\prime}\left(t_{m_{1}}^{1}\right), \ldots, Y^{(N)}\left(t_{1}^{N}\right), \ldots, Y^{(N)}\left(t_{m_{N}}^{N}\right)\right)
$$

are independent, $t_{1}^{0}, \ldots, t_{m_{0}}^{0}, t_{1}^{1}, \ldots, t_{m_{1}}^{1}, \ldots, t_{1}^{N}, \ldots, t_{m_{N}}^{N} \in[0, T], m_{0}, \ldots, m_{N} \geq$ 1 , then $X_{0}$ and $\left(Y_{0}, \ldots, Y_{N}\right)$ will be independent, as wanted.

We prove this assertion by induction on $N \geq 0$. For $N=0$, we already know that $X_{0}$ and $\left(Y\left(t_{1}^{0}\right), \ldots, Y\left(t_{m_{0}}^{0}\right)\right)$ are independent for each $t_{1}^{0}, \ldots, t_{m_{0}}^{0} \in[0, T]$, $m_{0} \geq 1$, because of the independence of $X_{0}$ and $Y$. We assume the assertion true for $N-1$ and we prove it for $N$. Write

$$
\begin{aligned}
& \left(Y\left(t_{1}^{0}\right), \ldots, Y\left(t_{m_{0}}^{0}\right), \ldots, Y^{(N-1)}\left(t_{1}^{N-1}\right), \ldots, Y^{(N-1)}\left(t_{m_{N-1}-1}^{N-1}\right), Y^{(N)}\left(t_{1}^{N}\right), \ldots, Y^{(N)}\left(t_{m_{N}}^{N}\right)\right) \\
= & \lim _{h \rightarrow 0}\left(Y\left(t_{1}^{0}\right), \ldots, Y\left(t_{m_{0}}^{0}\right), \ldots, Y^{(N-1)}\left(t_{1}^{N-1}\right), \ldots, Y^{(N-1)}\left(t_{m_{N-1}-1}^{N-1}\right),\right. \\
& \left.\frac{Y^{(N-1)}\left(t_{1}^{N}+h\right)-Y^{(N-1)}\left(t_{1}^{N}\right)}{h}, \ldots, \frac{Y^{(N-1)}\left(t_{m_{N}}^{N}+h\right)-Y^{(N-1)}\left(t_{m_{N}}^{N}\right)}{h}\right),
\end{aligned}
$$

where the limit is understood in $\mathrm{L}^{2}\left(\Omega ; \mathbb{R}^{m_{0}+m_{1}+\ldots+m_{N}}\right)$. By induction, $X_{0}$ is independent of each random vector

$$
\begin{aligned}
& \left(Y\left(t_{1}^{0}\right), \ldots, Y\left(t_{m_{0}}^{0}\right), \ldots, Y^{(N-1)}\left(t_{1}^{N-1}\right), \ldots, Y^{(N-1)}\left(t_{m_{N-1}-1}^{N-1}\right),\right. \\
& \left.\frac{Y^{(N-1)}\left(t_{1}^{N}+h\right)-Y^{(N-1)}\left(t_{1}^{N}\right)}{h}, \ldots, \frac{Y^{(N-1)}\left(t_{m_{N}}^{N}+h\right)-Y^{(N-1)}\left(t_{m_{N}}^{N}\right)}{h}\right) .
\end{aligned}
$$

This implies that $X_{0}$ and

$$
\left(Y\left(t_{1}^{0}\right), \ldots, Y\left(t_{m_{0}}^{0}\right), Y^{\prime}\left(t_{1}^{1}\right), \ldots, Y^{\prime}\left(t_{m_{1}}^{1}\right), \ldots, Y^{(N)}\left(t_{1}^{N}\right), \ldots, Y^{(N)}\left(t_{m_{N}}^{N}\right)\right)
$$

are independent, as wanted. 
An analogous result is satisfied if $\boldsymbol{H}_{\mathbf{1}}$ holds, instead of $\boldsymbol{H}_{\mathbf{0}}$. This concludes the remark.

If $\boldsymbol{H}_{\mathbf{0}}$ holds, we know the probability law of the random vector $\left(Y_{0}, \ldots, Y_{N}\right)$, and $X_{1}$ and $\left(Y_{0}, \ldots, Y_{N}\right)$ are independent, then the density function of $X_{N}(t)$ is computable: 2.14 becomes

$$
\begin{aligned}
& f_{X_{N}(t)}(x)=\mathbb{E}\left[f_{X_{0}}\left(\frac{x-\frac{\mathrm{e}^{-\omega_{0} \xi t} \sin \left(\omega_{1} t\right)}{\omega_{1}} X_{1}-\sum_{n=0}^{N} Y_{n} \int_{0}^{t} h(t-s) s^{n} \mathrm{~d} s}{\frac{\xi \mathrm{e}^{-\omega_{0} \xi t} \sin \left(\omega_{1} t\right)}{\sqrt{1-\xi^{2}}}+\mathrm{e}^{-\omega_{0} \xi t} \cos \left(\omega_{1} t\right)}\right)\right] \\
&=\frac{1}{\left|\frac{\xi \mathrm{e}^{-\omega_{0} \xi t} \sin \left(\omega_{1} t\right)}{\sqrt{1-\xi^{2}}}+\mathrm{e}^{-\omega_{0} \xi t} \cos \left(\omega_{1} t\right)\right|} \\
& \cdot \frac{1}{\left|\frac{\xi \mathrm{e}^{-\omega_{0} \xi t} \sin \left(\omega_{1} t\right)}{\sqrt{1-\xi^{2}}}+\mathrm{e}^{-\omega_{0} \xi t} \cos \left(\omega_{1} t\right)\right|} \\
& \cdot \int_{\mathbb{R}^{N+1}} \int_{\mathbb{R}} f_{X_{0}}\left(\frac{x-\frac{\mathrm{e}^{-\omega_{0} \xi t} \sin \left(\omega_{1} t\right)}{\omega_{1}} x_{1}-\sum_{n=0}^{N} y_{n} \int_{0}^{t} h(t-s) s^{n} \mathrm{~d} s}{\frac{\xi \mathrm{e}^{-\omega_{0} \xi t} \sin \left(\omega_{1} t\right)}{\sqrt{1-\xi^{2}}}+\mathrm{e}^{-\omega_{0} \xi t} \cos \left(\omega_{1} t\right)}\right) \\
& \cdot \mathbb{P}_{X_{1}}\left(\mathrm{~d} x_{1}\right) \mathbb{P}_{\left(Y_{0}, \ldots, Y_{N}\right)}\left(\mathrm{d} y_{0}, \ldots, \mathrm{d} y_{N}\right) .
\end{aligned}
$$

If $\boldsymbol{H}_{\mathbf{1}}$ holds, and $X_{0}$ and $\left(Y_{0}, \ldots, Y_{N}\right)$ are independent, (2.15) becomes

$$
\begin{aligned}
& f_{X_{N}(t)}(x) \\
& =\mathbb{E}\left[f_{X_{1}}\left(\frac{x-\left(\frac{\xi \mathrm{e}^{-\omega_{0} \xi t} \sin \left(\omega_{1} t\right)}{\sqrt{1-\xi^{2}}}+\mathrm{e}^{-\omega_{0} \xi t} \cos \left(\omega_{1} t\right)\right) X_{0}-\sum_{n=0}^{N} Y_{n} \int_{0}^{t} h(t-s) s^{n} \mathrm{~d} s}{\frac{\mathrm{e}^{-\omega_{0} \xi t} \sin \left(\omega_{1} t\right)}{\omega_{1}}}\right)\right] \\
& \frac{1}{\frac{\mathrm{e}^{-\omega_{0} \xi t}\left|\sin \left(\omega_{1} t\right)\right|}{\left|\omega_{1}\right|}} \\
& =\frac{1}{\frac{\mathrm{e}^{-\omega_{0} \xi t}\left|\sin \left(\omega_{1} t\right)\right|}{\left|\omega_{1}\right|}} \\
& \cdot \int_{\mathbb{R}^{N+1}} \int_{\mathbb{R}} f_{X_{1}}\left(\frac{x-\left(\frac{\xi \mathrm{e}^{-\omega_{0} \xi t} \sin \left(\omega_{1} t\right)}{\sqrt{1-\xi^{2}}}+\mathrm{e}^{-\omega_{0} \xi t} \cos \left(\omega_{1} t\right)\right) x_{0}-\sum_{n=0}^{N} y_{n} \int_{0}^{t} h(t-s) s^{n} \mathrm{~d} s}{\frac{\mathrm{e}^{-\omega_{0} \xi t} \sin \left(\omega_{1} t\right)}{\omega_{1}}}\right) \\
& \text { - } \mathbb{P}_{X_{0}}\left(\mathrm{~d} x_{0}\right) \mathbb{P}_{\left(Y_{0}, \ldots, Y_{N}\right)}\left(\mathrm{d} y_{0}, \ldots, \mathrm{d} y_{N}\right) .
\end{aligned}
$$

If the assumptions of Theorem 2.7 or Theorem 2.9 hold, then we can approximate $f_{X(t)}$ by using $\lim _{N \rightarrow \infty} f_{X_{N}(t)}(x)=f_{X(t)}(x)$.

In fact, a more general result than Theorem 2.9 can be proved in this setting: 
Theorem 2.15 The following statements hold:

- Assume $\boldsymbol{H}_{\mathbf{0}}$. Suppose that $f_{X_{0}}$ is a.e. continuous on $\mathbb{R}$ and $f_{X_{0}}(x) \leq a+$ $b x^{2}$ for a.e. $x \in \mathbb{R}$, for certain $a, b>0$. If, in addition, $\boldsymbol{H}_{\mathbf{1}}$ holds or some $Y_{n}$ is absolutely continuous, independent of $\left(X_{1}, Y_{0}, \ldots, Y_{n-1}, Y_{n+1}, \ldots\right)$ and with $\int_{0}^{t} h(t-s) s^{n} \mathrm{~d} s \neq 0$, then $\lim _{N \rightarrow \infty} f_{X_{N}(t)}(x)=f_{X(t)}(x)$ for all $x \in \mathbb{R}$, being $f_{X_{N}(t)}(x)$ and $f_{X}(x)$ defined by (2.14) and (2.5), respectively.

- Assume $\boldsymbol{H}_{\mathbf{1}}$. Suppose that $f_{X_{1}}$ is a.e. continuous on $\mathbb{R}$ and $f_{X_{1}}(x) \leq a+$ bx $x^{2}$ for a.e. $x \in \mathbb{R}$, for certain $a, b>0$. If, in addition, $\boldsymbol{H}_{\mathbf{0}}$ holds or some $Y_{n}$ is absolutely continuous, independent of $\left(X_{0}, Y_{0}, \ldots, Y_{n-1}, Y_{n+1}, \ldots\right)$ and with $\int_{0}^{t} h(t-s) s^{n} \mathrm{~d} s \neq 0$, then $\lim _{N \rightarrow \infty} f_{X_{N}(t)}(x)=f_{X(t)}(x)$ for all $x \in \mathbb{R}$, being $f_{X_{N}(t)}(x)$ and $f_{X}(x)$ defined by 2.15) and (2.6), respectively.

Proof. Analogous to Theorem 2.11.

If $\boldsymbol{H}_{\mathbf{0}}$ and $\boldsymbol{H}_{\mathbf{1}}$ do not hold, we can consider the hypothesis

$\boldsymbol{H}_{\mathbf{3}}$ : There is an $n_{0} \geq 0$ such that $Y_{n_{0}}$ is absolutely continuous, $\int_{0}^{t} h(t-$ $s) s^{n_{0}} \mathrm{~d} s \neq 0, Y_{n_{0}}$ and $\left(X_{0}, X_{1}, Y_{0}, \ldots, Y_{n_{0}-1}, Y_{n_{0}+1}, \ldots, Y_{N}\right)$ are independent, for each $N \geq 0$.

In such a case, the random variable $Z_{1}=Y_{n_{0}} \int_{0}^{t} h(t-s) s^{n_{0}} \mathrm{~d} s$ is absolutely continuous, with density function

$$
f_{Z_{1}}(z)=f_{Y_{n_{0}}}\left(\frac{z}{\int_{0}^{t} h(t-s) s^{n_{0}} \mathrm{~d} s}\right) \frac{1}{\left|\int_{0}^{t} h(t-s) s^{n_{0}} \mathrm{~d} s\right|} .
$$

By Lemma 2.3, for $N \geq n_{0}$,

$$
\begin{aligned}
& f_{X_{N}(t)}(x)=\frac{1}{\left|\int_{0}^{t} h(t-s) s^{n_{0}} \mathrm{~d} s\right|} \\
& \cdot \mathbb{E}\left[f_{Y_{n_{0}}}\left(\frac{x-\left(\frac{\xi^{-\omega_{0} \xi t} \sin \left(\omega_{1} t\right)}{\sqrt{1-\xi^{2}}}+\mathrm{e}^{-\omega_{0} s t} \cos \left(\omega_{1} t\right)\right) X_{0}-\frac{\mathrm{e}^{-\omega_{0} \delta t} \sin \left(\omega_{1} t\right)}{\omega_{1}} X_{1}-\sum_{n \neq n_{0}}^{N} Y_{n} \int_{0}^{t} h(t-s) s^{n} \mathrm{~d} s}{\int_{0}^{t} h(t-s) s^{n_{0}} \mathrm{~d} s}\right)\right] .
\end{aligned}
$$

With analogous proofs to those of Theorem 2.7 and Theorem 2.9, one arrives at the following results:

Theorem 2.16 Assume $\boldsymbol{H}_{3}$. If $f_{Y_{n_{0}}}$ is continuous on $\mathbb{R}$ and $f_{Y_{n_{0}}}(x) \leq a+b x^{2}$, for certain $a, b>0$, then $\lim _{N \rightarrow \infty} f_{X_{N}(t)}(x)=f_{X(t)}(x)$ for all $x \in \mathbb{R}$, being $f_{X_{N}(t)}(x)$ given by 2.26) and $f_{X(t)}(x)$ given by 2.26) with $N=\infty$. 
Theorem 2.17 Assume $\boldsymbol{H}_{\mathbf{3}}$. Suppose that $f_{Y_{n_{0}}}$ is a.e. continuous on $\mathbb{R}$ and $f_{Y_{n_{0}}}(x) \leq a+b x^{2}$ for a.e. $x \in \mathbb{R}$, for certain $a, b>0$. If, in addition, $\boldsymbol{H}_{\mathbf{0}}$ holds or $\boldsymbol{H}_{\mathbf{1}}$ holds or some other $Y_{n}, n \neq n_{0}$, satisfies $\boldsymbol{H}_{\mathbf{3}}$, then $\lim _{N \rightarrow \infty} f_{X_{N}(t)}(x)=$ $f_{X(t)}(x)$ for all $x \in \mathbb{R}$, being $f_{X_{N}(t)}(x)$ given by 2.26) and $f_{X(t)}(x)$ given by (2.26) with $N=\infty$.

Remark 2.18 Hypotheses $\boldsymbol{H}_{\mathbf{0}}, \boldsymbol{H}_{\mathbf{1}}, \boldsymbol{H}_{\mathbf{2}}$ and $\boldsymbol{H}_{\mathbf{3}}$ are not necessary to have $X(t)$ absolutely continuous. Indeed, by [140], there exists a singular continuous measure $\lambda$ such that $\lambda * \lambda$ is absolutely continuous (here $*$ stands for the convolution operator). Fix $\bar{t} \in[0, T]$ such that

$$
\frac{\xi \sin \left(\omega_{1} \bar{t}\right)}{\sqrt{1-\xi^{2}}}+\cos \left(\omega_{1} \bar{t} \neq 0, \quad \sin \left(\omega_{1} \bar{t} \neq 0 .\right.\right.
$$

Take a pair of independent random variables $X_{0}$ and $X_{1}$ such that

$$
\left(\frac{\xi \mathrm{e}^{-\omega_{0} \xi \bar{t}} \sin \left(\omega_{1} \bar{t}\right)}{\sqrt{1-\xi^{2}}}+\mathrm{e}^{-\omega_{0} \xi \bar{t}} \cos \left(\omega_{1} \bar{t}\right)\right) X_{0} \sim \lambda, \quad \frac{\mathrm{e}^{-\omega_{0} \xi \bar{t}} \sin \left(\omega_{1} \bar{t}\right)}{\omega_{1}} X_{1} \sim \lambda .
$$

Let $Y=0$. Then the solution stochastic process $X(\bar{t})$ has distribution $\lambda * \lambda$, which is absolutely continuous. However, $\boldsymbol{H}_{\mathbf{0}}$ and $\boldsymbol{H}_{\mathbf{1}}$ do not hold, because $\boldsymbol{X}_{0}$ and $X_{1}$ are not absolutely continuous, respectively. Thus, we have a pathological example. In these cases, at least to our knowledge, it is not possible to know the probability density function of the solution stochastic process.

\subsection{Applications}

In this section, we showcase the proposed approach on several examples where we apply our theoretical findings to particular random problems 2.1). The examples will cover a wide variety of situations which are of mathematical and physical interest. The main objective will be to test the methodology reported in this chapter.

For the sake of clarity, throughout Examples 2.21 2.25 we will fix the constants in (2.1): we choose the upper time $T=1$, the damping ratio $\xi=1 / 2$ and the natural frequency $\omega_{0}=\pi / 2$. Then, according to (2.4), $\omega_{1}=\omega_{0} \sqrt{1-\xi^{2}}=$ $\pi \sqrt{3} / 4$. Notice that, as $\omega_{1} \in(0, \pi / 2)$, we have $\sin \left(\omega_{1} t\right)>0$ and $\cos \left(\omega_{1} t\right)>0$, for $t \in(0,1]=(0, T]$. Then

$$
\frac{\xi \sin \left(\omega_{1} t\right)}{\sqrt{1-\xi^{2}}}+\cos \left(\omega_{1} t\right)>0
$$

on $[0,1]$ and $h(t)>0$ on $(0,1]$. We will work with independent $X_{0}, X_{1}$ and $Y$. Physically, the initial position, the initial velocity and the harmonic excitation 
are independent. On the other hand, to ensure the inequality $f(x) \leq a+b x^{2}$ for a specific density $f$, we will take $f$ bounded. Notice that this boundedness restriction covers any possible situation in practice, as a random variable with an unbounded density function may be truncated to achieve the boundedness condition but maintaining its main probabilistic features [77]. Moreover, truncation does not affect almost everywhere continuity of the density, which has been a very important hypothesis in some of the previous theorems.

Finally, to obtain/plot density functions given by an expectation expression (2.5), 2.6), (2.11), (2.14), etc.), we will use Monte Carlo simulation.

Example 2.19 In this example we determine the probability density function of the response of a pendulum differential equation model to earthquake type random disturbances. Reference [9] justifies the use of equation (2.1) to model the response. Soong [125, Example 7.3] provides a summary of the results obtained in [9] and focuses on the mathematical properties of the random differential equation model, by computing the expectation and covariances, but not the probability density function. Reference [64] is a continuation of the investigation from [9]. On our part, our method to find the probability density function of the response will consist in a direct application of Lemma 2.3.

In [9, 64], [125, Example 7.3], a simple structure approximating a linear onestory building is considered. The goal is to analyze the response of this structure to an earthquake type random disturbance with ground acceleration

$$
Y(t)=\sum_{j=1}^{n} t a_{j} \mathrm{e}^{-\alpha_{j} t} \cos \left(w_{j} t+\theta_{j}\right), \quad t \geq 0 .
$$

It is assumed that $a_{j}, \alpha_{j}$ and $w_{j}$ are constant, while $\theta_{1}, \ldots, \theta_{n}$ are independent random variables with Uniform $(0,2 \pi)$ distribution. The term $Y(t)$ has this particular form because it takes the usual appearance of earthquake accelerogram records when $n$ is sufficiently large. Horizontal displacement of the roof of the structure is assumed due to ground motion. Let $X(t)$ be the relative horizontal displacement of the roof with respect to the ground (see [125] for further detailed physical justification). We assume that the roof is at rest at $t=0: X(0)=X_{0}=0$ and $\dot{X}(0)=X_{1}=0$. By [9], the relative displacement of the roof, $X(t)$, is governed by the pendulum differential equation

$$
\ddot{X}(t)+2 \omega_{0} \xi \dot{X}(t)+\omega_{0}^{2} X(t)=-Y(t) .
$$

The solution of this model is given by

$$
X(t)=-\int_{0}^{t} h(t-s) Y(s) \mathrm{d} s,
$$


where the impulse response $h$ is given by (2.4).

In order to find the probability density function of $X(t)$, we rewrite $Y(t)$ as follows:

$Y(t)=t a_{1} \mathrm{e}^{-\alpha_{1} t}\left(\cos \left(w_{1} t\right) \cos \left(\theta_{1}\right)-\sin \left(w_{1} t\right) \sin \left(\theta_{1}\right)\right)+\sum_{j=2}^{n} t a_{j} \mathrm{e}^{-\alpha_{j} t} \cos \left(w_{j} t+\theta_{j}\right)$.

Then $X(t)$ can be written as $X(t)=Z_{1}+Z_{2}$, where

$$
\begin{aligned}
Z_{1} & =\left(-\int_{0}^{t} h(t-s) s a_{1} \mathrm{e}^{-\alpha_{1} s} \cos \left(w_{1} s\right) \mathrm{d} s\right) \cos \left(\theta_{1}\right) \\
& +\left(\int_{0}^{t} h(t-s) s a_{1} \mathrm{e}^{-\alpha_{1} s} \sin \left(w_{1} s\right) \mathrm{d} s\right) \sin \left(\theta_{1}\right), \\
Z_{2} & =-\sum_{j=2}^{n} \int_{0}^{t} h(t-s) s a_{j} \mathrm{e}^{-\alpha_{j} s} \cos \left(w_{j} s+\theta_{j}\right) \mathrm{d} s .
\end{aligned}
$$

Since $\theta_{1}, \ldots, \theta_{n}$ are independent, for each fixed $t \geq 0$ the random variables $Z_{1}$ and $Z_{2}$ are also independent, and $Z_{1}$ is absolutely continuous, whose density function $f_{Z_{1}}$ can be obtained numerically by using that it is a transformation of $\theta_{1}$. By Lemma 2.3, the response $X(t)$ is absolutely continuous and its density function is given by $f_{X(t)}(x)=\mathbb{E}\left[f_{Z_{1}}\left(x-Z_{2}\right)\right]$. This expectation can be approximated by means of Monte Carlo simulation.

In order to obtain a graphical representation of the probability density function of $X(t)$, let us fix the following values for the constants: $\omega_{0}=20, \xi=0.05$, $n=20, \alpha_{i}=0.333, a_{i}=0.5$ and $w_{i}=15$, for $i=1, \ldots, 20$. In Figure 2.1, we plot $f_{X(t)}(x)$ for $t=0.1$.

Example 2.20 White noise processes are of great interest in random vibrations. The probabilistic characteristics of dynamical systems with white noise inputs have been examined [81, 98, 32]. In this example, we consider the response of a mass-spring linear oscillator to a white noise random excitation, with governing equation $\ddot{X}(t)+\omega_{0}^{2} X(t)=Y(t)$, see [125, Example 7.1]. This is a particular case of (2.1) with damping ratio $\xi=0$. The source term $Y(t)$ is the formal derivative of a Brownian motion process, therefore the initial value problem (2.1) takes the form of a stochastic differential equation of Itô type. It is assumed that $X_{0}, X_{1}$ and $Y$ are independent.

As discussed in Subsection 2.2.1, the probability density function of the response $X(t)$ is expressed by (2.11), being $\mu_{I}(t)=0$ and $\sigma_{I}(t)^{2}=\int_{0}^{t} h(s)^{2} \mathrm{~d} s$, where the impulse response $h$ is given by (2.4). 


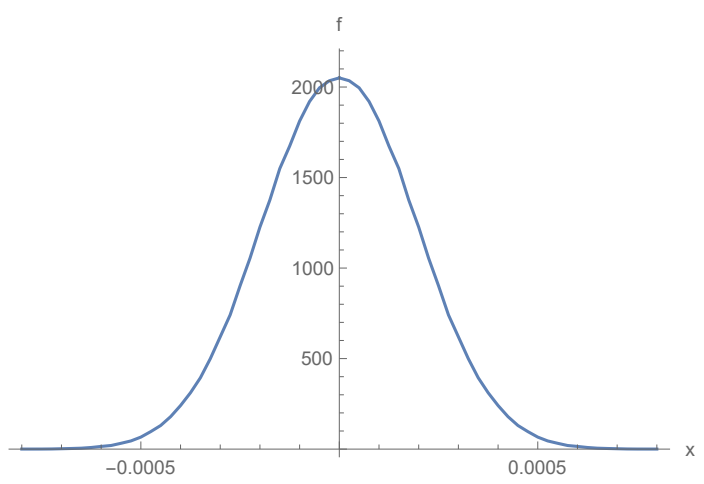

Figure 2.1: Density function $f_{X(t)}(x)$ for $t=0.1$ in Example 2.19.

For the numerical experiment, let us fix the frequency $\omega_{0}=1$, the initial position $X_{0}=0$ and the initial velocity $X_{1} \sim$ Triangular $(-0.1,0.1)$. In Figure 2.2, we depict the density function of $X(t)$ at $t=0.8$.

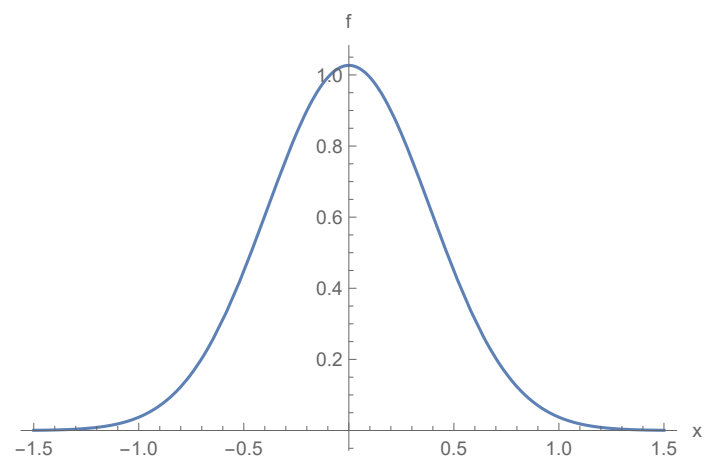

Figure 2.2: Density function $f_{X(t)}(x)$ for $t=0.8$ in Example 2.20 .

Example 2.21 Consider model 2.1 with fixed initial position $X_{0}=-1$, initial velocity $X_{1} \sim \operatorname{Poisson}(5)$ and torcing term $Y(t)=A \sim \operatorname{Exponential}(4)$, for all $t \in[0,1](Y(t)$ is a constant random variable as a function of $t$, i.e., a steady-state random excitation). These random variables are assumed to be independent. Using expression (2.26) with $N=n_{0}=0$ and $Y_{0}=A$, and taking into account that $\int_{0}^{t} h(t-s) s^{n_{0}} \mathrm{~d} s=\int_{0}^{t} h(t-s) \mathrm{d} s>0$, we deduce that the probability density function of the response stochastic process $X(t)$ 
is given by

$$
\begin{aligned}
f_{X(t)}(x) & \frac{1}{\left|\int_{0}^{t} h(t-s) \mathrm{d} s\right|} \mathbb{E}\left[f_{A}\left(\frac{x-\left(\frac{\xi \mathrm{e}^{-\omega_{0} \xi t} \sin \left(\omega_{1} t\right)}{\sqrt{1-\xi^{2}}}+\mathrm{e}^{-\omega_{0} \xi t} \cos \left(\omega_{1} t\right)\right) X_{0}-\frac{\mathrm{e}^{-\omega_{0} \xi t} \sin \left(\omega_{1} t\right)}{\omega_{1}} X_{1}}{\int_{0}^{t} h(t-s) \mathrm{d} s}\right)\right],
\end{aligned}
$$

for $t \in(0,1]$. In Figure 2.3 , we show the graph of $f_{X(0.2)}(x)$, for $-1.5 \leq x \leq 1.5$.

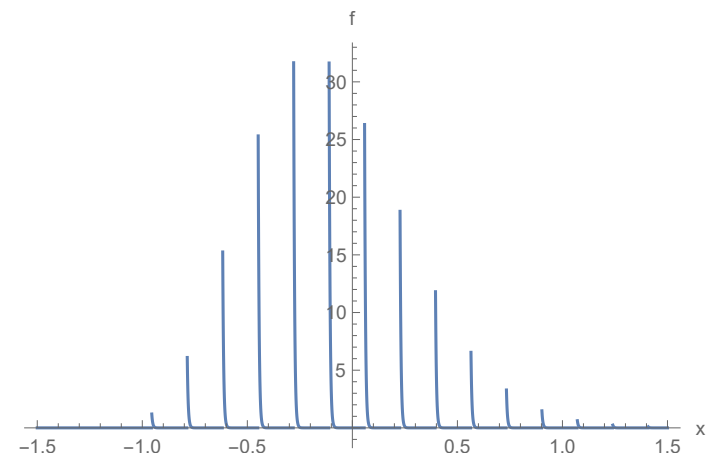

Figure 2.3: Density function $f_{X(0.2)}(x)$ in Example 2.21.

Example 2.22 Let us consider model (2.1) with initial position $X_{0}$ having a Cantor distribution [66] and no initial motion, $X_{1}=0$. Recall that the Cantor distribution is defined by having as cumulative distribution function the Cantor staircase function. The Cantor distribution can be seen in the following way:

$$
X_{0}=2 \sum_{k=1}^{\infty} \frac{A_{k}}{3^{k}},
$$

where $A_{1}, A_{2}, \ldots$ are independent random variables with $\operatorname{Bernoulli}(0.5)$ distribution and the series converges a.s. Notice that $X_{0}$ is not an absolutely continuous random variable, hence hypothesis $\boldsymbol{H}_{\mathbf{0}}$ does not hold. We take the forcing term $Y(t)$ as a standard Brownian motion on [0,1] [89, Def. 5.11]. It is assumed that $X_{0}, X_{1}$ and $Y$ are independent.

Using formulas 2.7 and 2.8 and taking into account that $\mathbb{E}[Y(t)]=0$ and that $\operatorname{Cov}[Y(t), Y(s)]=\min \{t, s\}$, we compute $\mu_{I}(t)=0$ and $\sigma_{I}(t)^{2}=$ $\int_{0}^{t} \int_{0}^{t} h\left(t-s_{1}\right) h\left(t-s_{2}\right) \min \left\{s_{1}, s_{2}\right\} \mathrm{d} s_{1} \mathrm{~d} s_{2}$. 
According to Proposition 2.5, we have that the response $X(t)$ is absolutely continuous, with density function given by (2.11). In Figure 2.4, we have plotted the graph of $f_{X(0.1)}(x)$ and $f_{X(0.8)}(x)$. For $t=0.1$, we observe a big influence of the initial condition $X_{0}$. For $t=0.8$, the influence of $X_{0}$ seems to be dispelled, since $f_{X(0.8)}(x)$ is smoother.
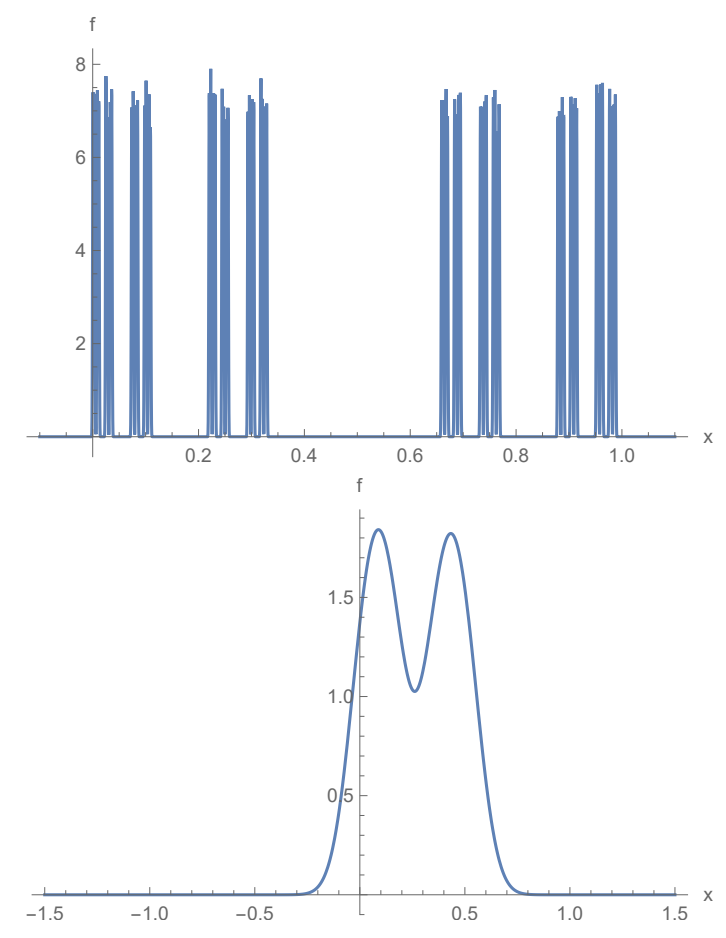

Figure 2.4: First: Density function $f_{X(0.1)}(x)$. Second: Density function $f_{X(0.8)}(x)$. Both in the context of Example 2.22 and interpreting the Brownian motion $Y(t)=B(t)$ as a Gaussian stochastic process.

We may also see the Brownian motion as a Karhunen-Loève expansion [89, Exercise 5.12]:

$$
Y(t)=\sum_{j=1}^{\infty} \frac{\sqrt{2}}{\left(j-\frac{1}{2}\right) \pi} \sin \left(t\left(j-\frac{1}{2}\right) \pi\right) \xi_{j},
$$

where $\left\{\xi_{j}\right\}_{j=1}^{\infty}$ is a sequence of independent random variables with $\operatorname{Normal}(0,1)$ distribution. The series converges in $\mathrm{L}^{2}([0,1] \times \Omega)$. 
By Theorem 2.12, the density function of $X_{N}(t)$ (given by (2.25) with $J=N$ ) converges pointwise to $f_{X(t)}(x)$, for $t \in(0,1]$ (given by $(2.25)$ with $J=\infty$ ). In Figure 2.5, we have plotted the graph of $f_{X_{15}(0.1)}(x)$ and $f_{X_{15}(0.8)}(x)(N=15)$. Notice that these densities approximate accurately the exact densities from Figure 2.4, as Theorem 2.12 states.
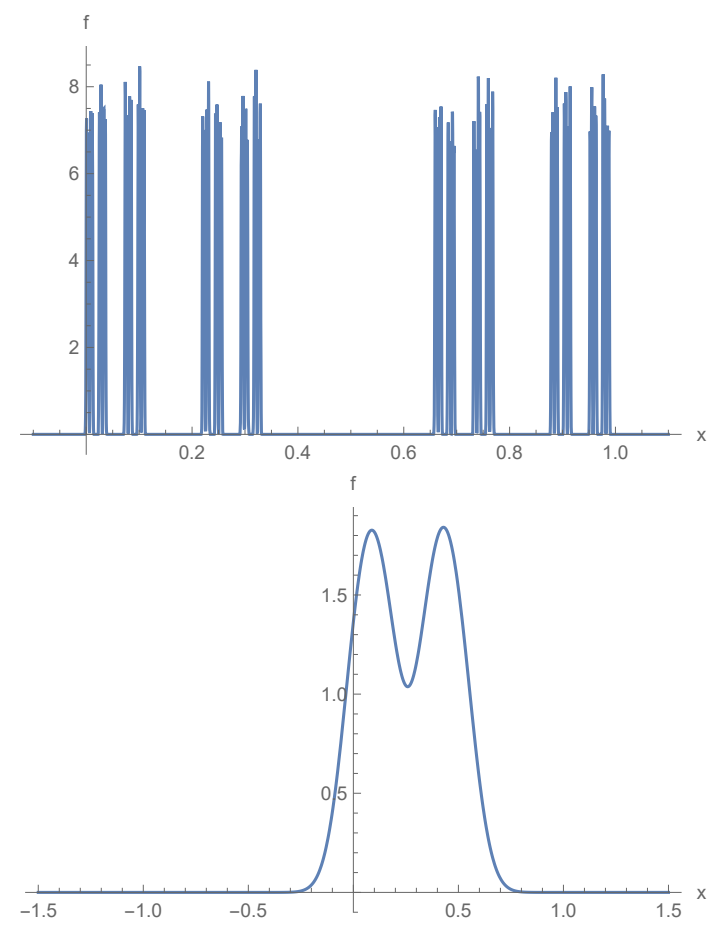

Figure 2.5: First: Density function $f_{X_{15}(0.1)}(x)$. Second: Density function $f_{X_{15}(0.8)}(x)$. Both in the context of Example 2.22 and interpreting the Brownian motion $Y(t)=B(t)$ via its Karhunen-Loève expansion.

Example 2.23 Consider (2.1), $X_{0} \sim \operatorname{Exponential(3),~} X_{1} \sim \operatorname{Binomial}(7,0.31)$ and

$$
Y(t)=\sum_{j=1}^{\infty} \frac{\sqrt{2}}{j \pi} \sin (t j \pi) \xi_{j} .
$$

The series is understood in $\mathrm{L}^{2}([0,1] \times \Omega)$ and $\left\{\xi_{j}\right\}_{j=1}^{\infty}$ is a sequence of independent random variables with $\operatorname{Uniform}(-\sqrt{3}, \sqrt{3})$ distribution. This is a Karhunen-Loève expansion. It is assumed that $X_{0}, X_{1}$ and $Y$ are independent. 
Since $\phi_{1}(t)=\sin (t \pi)>0$ on $0<t<1$ and $h(t)>0$ on $0<t \leq 1$, then $\int_{0}^{t} h(t-s) \phi_{1}(s) \mathrm{d} s>0$, for $t \in(0,1]$. As a consequence, hypotheses $\boldsymbol{H}_{\mathbf{0}}$ and $\boldsymbol{H}_{\mathbf{2}}$ hold. By Theorem 2.11 or Theorem 2.13, $\lim _{N \rightarrow \infty} f_{X_{N}(t)}(x)=f_{X(t)}(x)$, for $t \in(0,1]$ and $x \in \mathbb{R}$.

In Figure 2.6 we have plotted $f_{X_{N}(0.5)}(x)$ for $N=1,2,3,4,5,6$. We observe convergence, since a small or nearly no variation of the density functions is noticed at different values of truncation order $N$. This convergence agrees with Theorem 2.11 and Theorem 2.13 .

Example 2.24 Consider (2.1) with $X_{0} \sim \operatorname{Gamma}(2,1), X_{1} \sim \operatorname{Beta}(1 / 2,1 / 2)$ and

$$
Y(t)=\sum_{j=1}^{\infty} \frac{\sqrt{2}}{j \pi} \sin (t j \pi) \xi_{j},
$$

where the series is understood in $\mathrm{L}^{2}([0,1] \times \Omega)$ and $\left\{\xi_{j}\right\}_{j=1}^{\infty}$ is a sequence of independent random variables with Uniform $\{-1,1\}$ distribution (discrete distribution with $\left.\mathbb{P}\left(\xi_{j}=-1\right)=\mathbb{P}\left(\xi_{j}=1\right)=1 / 2\right)$. This is a Karhunen-Loève expansion. It is assumed that $X_{0}, X_{1}$ and $Y$ are independent.

By Theorem 2.7. $\lim _{N \rightarrow \infty} f_{X_{N}(t)}(x)=f_{X(t)}(x)$, for $t \in[0,1]$ and $x \in \mathbb{R}$. In Figure 2.7 we plot $f_{X_{N}(0.5)}(x)$ for $N=1,2,3,4,5,6$. Convergence seems to be achieved, since nearly no variation of the density functions is noticed at different values of truncation order $N$. This fact agrees with Theorem 2.7 .

Example 2.25 Consider (2.1) with $X_{0} \sim$ Negative Binomial $(7,0.31)$ and $X_{1}$ absolutely continuous with density function given by $f_{X_{1}}(x)=\sqrt{2} /\left(\pi\left(1+x^{4}\right)\right)$, $x \in \mathbb{R}$. Take

$$
Y(t)=\sum_{n=1}^{\infty} \frac{A_{n}}{n} t^{n},
$$

for $t \in[0,1]$, where $A_{1}, A_{2}, \ldots$ are independent random variables, Poisson(5) distributed. Notice that the sum is well-defined in $\mathrm{L}^{2}([0,1] \times \Omega)$ : indeed,

$$
\left\|\frac{A_{n}}{n} t^{n}\right\|_{\mathrm{L}^{2}([0,1] \times \Omega)}=\frac{C}{n \sqrt{2 n+1}}
$$

where $C$ is the 2 -norm of a Poisson(5) random variable, therefore

$$
\sum_{n=1}^{\infty}\left\|\frac{A_{n}}{n} t^{n}\right\|_{\mathrm{L}^{2}([0,1] \times \Omega)}<\infty .
$$

It is assumed that $X_{0}, X_{1}, A_{1}, A_{2}, \ldots$ are independent. 

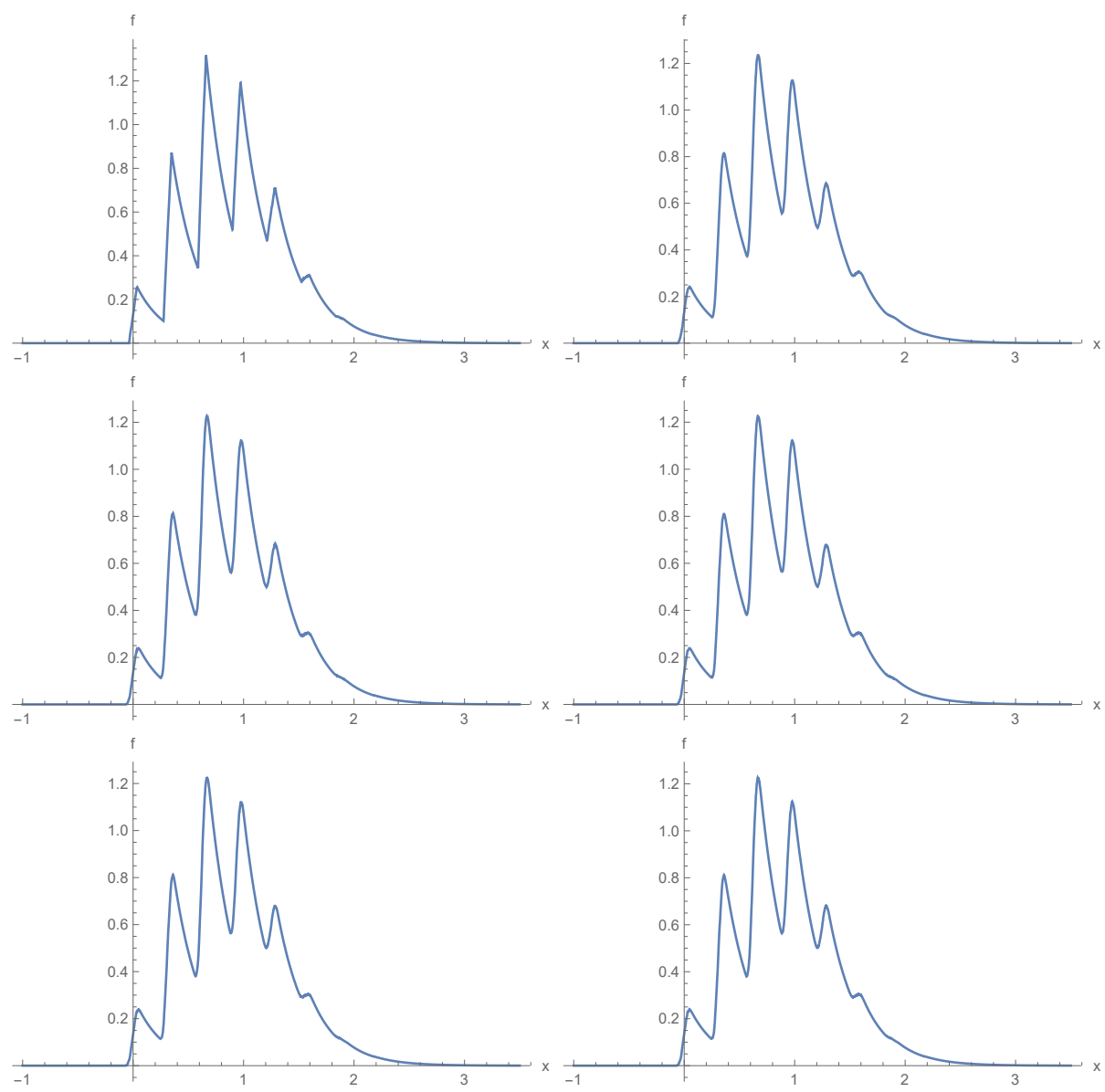

Figure 2.6: Density function $f_{X_{N}(0.5)}(x)$ for $N=1$ (up left), $N=2$ (up right), $N=3$ (center left), $N=4$ (center right), $N=5$ (down left) and $N=6$ (down right) in Example 2.23 

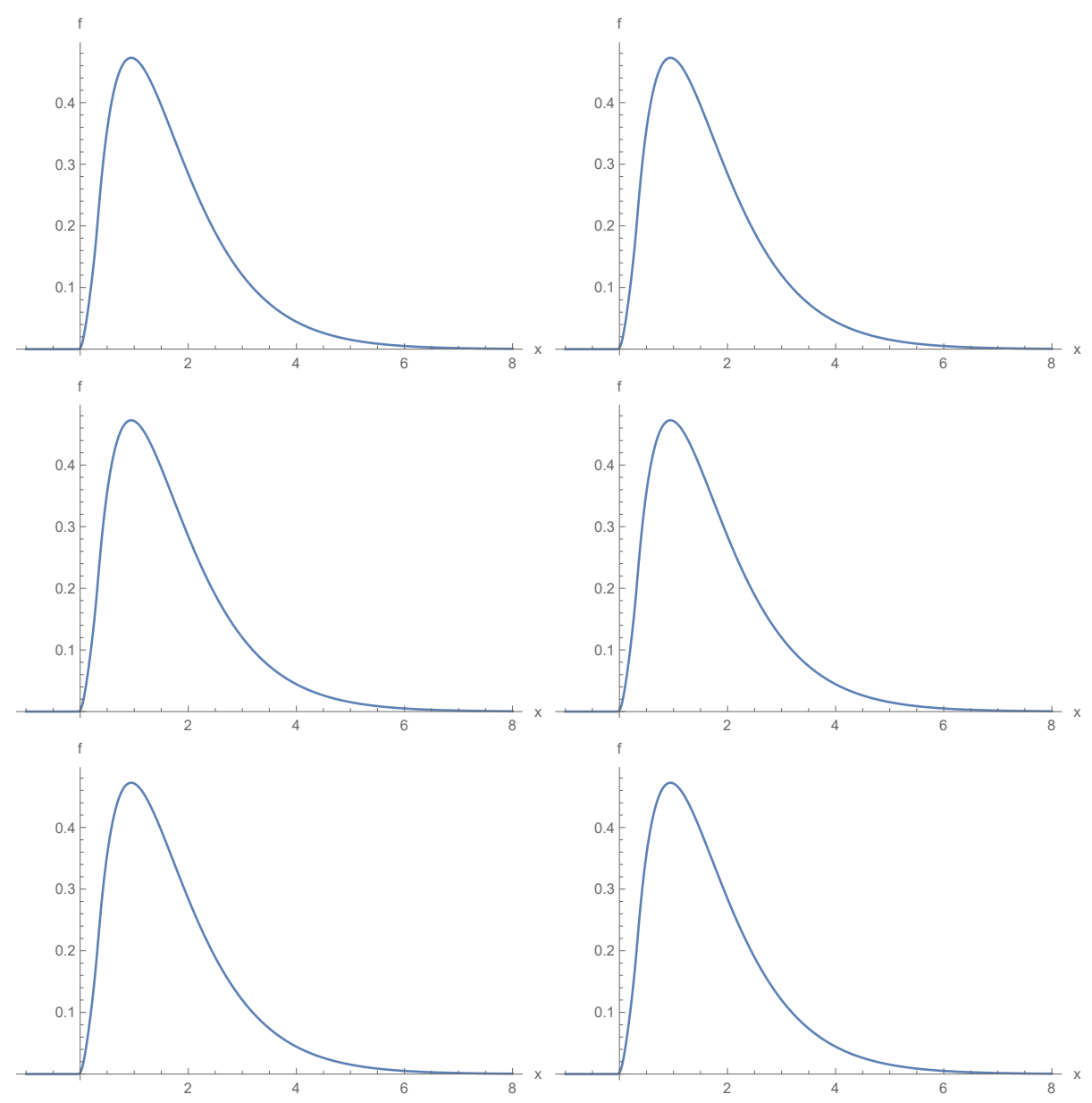

Figure 2.7: Density function $f_{X_{N}(0.5)}(x)$ for $N=1$ (up left), $N=2$ (up right), $N=3$ (center left), $N=4$ (center right), $N=5$ (down left) and $N=6$ (down right) in Example 2.24 
By Theorem 2.7. $\lim _{N \rightarrow \infty} f_{X_{N}(t)}(x)=f_{X(t)}(x)$, for $t \in(0,1]$ and $x \in \mathbb{R}$. In Figure 2.8, we plot $f_{X_{N}(0.3)}(x)$ for $N=1,2,3,4,5,6$. We observe convergence, since virtually the same density functions are plotted at different values of truncation order $N$.
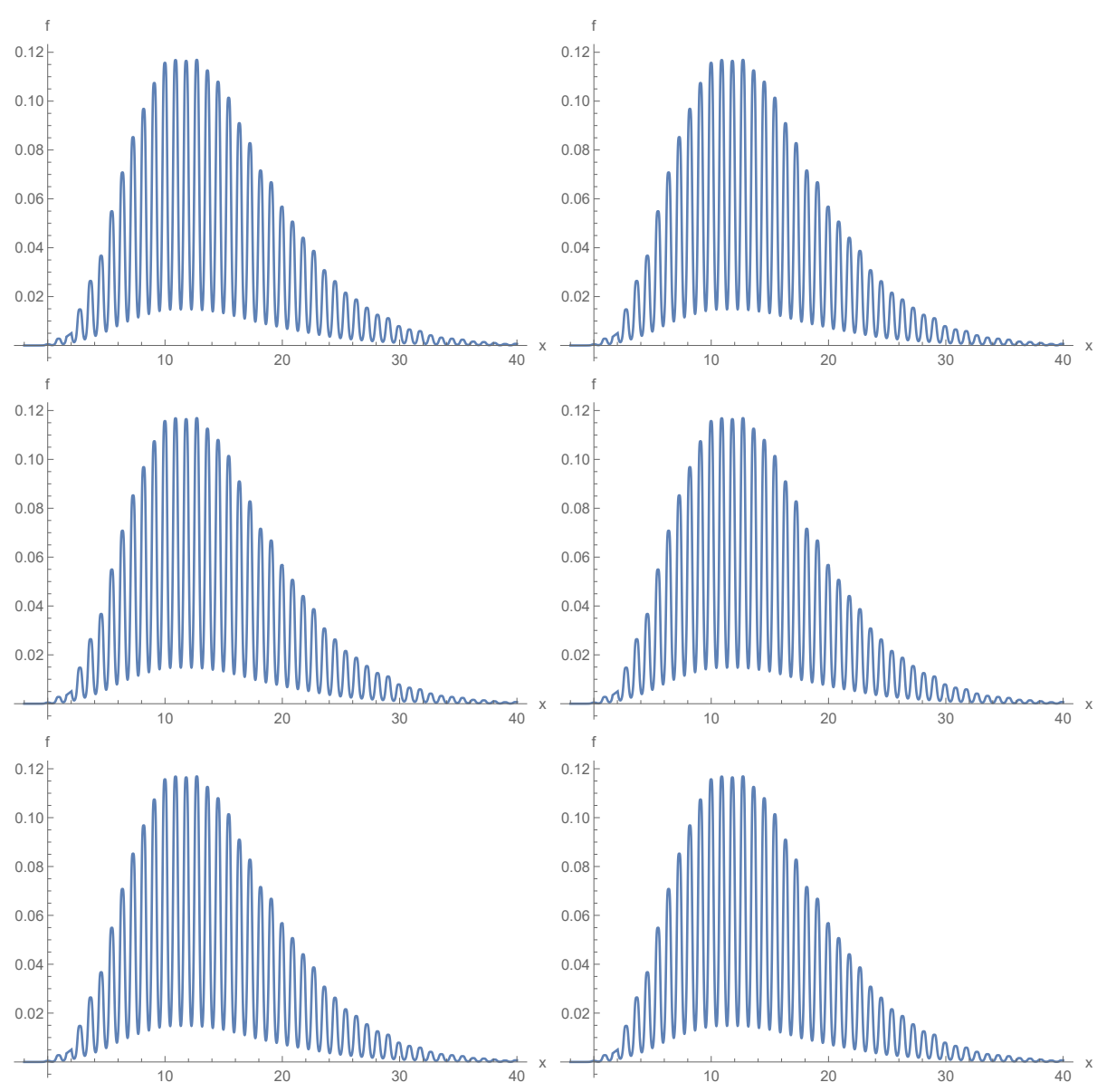

Figure 2.8: Density function $f_{X_{N}(0.3)}(x)$ for $N=1$ (up left), $N=2$ (up right), $N=3$ (center left), $N=4$ (center right), $N=5$ (down left) and $N=6$ (down right) in Example 2.25 


\subsection{Conclusions}

In this chapter we have provided a comprehensive probabilistic analysis of the damped pendulum differential equation in the case that the initial conditions (position, $X_{0}$, and velocity, $X_{1}$ ) are random variables and the forcing term, $Y(t)$, is a stochastic process. To the best of our knowledge, a major difference of our contribution with respect to the ones available in the existing literature is that we have provided exact or approximate expressions for the probability density function of the solution stochastic process of this important problem in Physics. Our achievement contrasts with other studies, where the goal is merely to construct exact or approximate expressions for the mean and the variance of the solution process. We think that a strong point of our contribution is the wide range of scenarios studied with respect to the forcing term $Y(t)$ as well as the generality of our analysis. In particular, the important cases where $Y(t)$ is Gaussian (including the White noise process) or $Y(t)$ can be represented via Karhunen-Loève expansion or via a mean square convergent random power series have been fully addressed. Furthermore, the study has included a detailed discussion with regard to the hypotheses assumed on the input data $\left(X_{0}, X_{1}, Y(t)\right)$ to establish our findings. The generality of such hypotheses in practical situations has been illustrated throughout a wide variety of numerical examples. The analysis performed throughout this chapter may be very useful in dealing with other random differential equations in future contributions.

\section{Acknowledgements}

This work has been supported by the Spanish Ministerio de Economía y Competitividad grant MTM2017-89664-P.

The main results of this chapter have been published in [17]. 



\section{Chapter 3}

\section{Approximation of the density function of random non-autonomous logistic-type differential equations}

In this chapter, we address the problem of approximating the probability density function of the following random logistic differential equation: $P^{\prime}(t, \omega)=A(t, \omega)(1-P(t, \omega)) P(t, \omega), t \in\left[t_{0}, T\right], P\left(t_{0}, \omega\right)=P_{0}(\omega)$, where $\omega$ is any outcome in the sample space $\Omega$. In the recent contribution [Cortés JC, et al. Commun Nonlinear Sci Numer Simulat 2019; 72: 121-138], the authors imposed conditions on the diffusion coefficient $A(t)$ and on the initial condition $P_{0}$ to approximate the density function $f_{1}(p, t)$ of $P(t): A(t)$ is expressed as a Karhunen-Loève expansion with absolutely continuous random coefficients that have certain growth and are independent of the absolutely continuous random variable $P_{0}$, and the density of $P_{0}, f_{P_{0}}$, is Lipschitz on $(0,1)$. In this chapter, we tackle the problem in a different manner, by using probability tools that allow the hypotheses to be less restrictive. We only suppose that $A(t)$ is expanded on $\mathrm{L}^{2}\left(\left[t_{0}, T\right] \times \Omega\right)$, so that we include other expansions such as random power series. We only require absolute continuity for $P_{0}$, so that $A(t)$ may be discrete or singular, due to a modified version of the random variable transformation technique. For $f_{P_{0}}$, only almost everywhere continuity and boundedness on $(0,1)$ are needed. We construct an approximating sequence $\left\{f_{1}^{N}(p, t)\right\}_{N=1}^{\infty}$ of density functions in terms of expectations that tends to $f_{1}(p, t)$ pointwise. Numerical examples illustrate our theoretical results. 


\subsection{Introduction and motivation}

In the recent paper [42, the authors studied the random non-autonomous logistic differential equation problem on a real interval $\left[t_{0}, T\right]$ :

$$
\left\{\begin{array}{l}
P^{\prime}(t, \omega)=A(t, \omega)(1-P(t, \omega)) P(t, \omega), \quad t \in\left[t_{0}, T\right], \\
P\left(t_{0}, \omega\right)=P_{0}(\omega) .
\end{array}\right.
$$

In this setting, we are assuming an underlying complete probability space $(\Omega, \mathcal{F}, \mathbb{P})$, where $\Omega$ is the sample space that consists of outcomes $\omega$ (which might usually be omitted in evaluations), $\mathcal{F} \subseteq 2^{\Omega}$ is the $\sigma$-algebra of events, and $\mathbb{P}$ is the probability measure. The initial condition $P_{0}$ is a random variable and the diffusion coefficient $A(t)$ is a stochastic process. In principle, these random data may take any probability distribution. The solution $P(t)$ to $(3.1)$ is also a stochastic process, whose sample paths solve the deterministic version of (3.1). General random differential equation problems have been profusely studied in several contributions, see for example [125, 129, 6, 104].

This initial value problem (3.1) corresponds to Verhulst model [136, 102], which extends Malthusian growth model by means of a carrying capacity when there is lack of nutrients and competition between species as time passes. The initial condition $P_{0}$ and the solution $P(t)$ represent proportions in $(0,1)$ of a given population (the carrying capacity due to resources constrains is therefore $1)$, thus their supports are restricted to $(0,1)$. The diffusion coefficient $A(t)$ is the reproduction parameter, with support that may take values on the whole $\mathbb{R}$ (although for growth modeling the reproductive parameter is positive). The outcome $\omega \in \Omega$ takes into account the variability in the population growth due to complex factors (environmental, genetic, etc.), error measurements, lack of information, etc. whose nature may be modeled as random. Some studies on the logistic differential equation with uncertainties may be found in [40, 88,

In dealing with random differential equation problems, a major goal is the computation of the (first) probability density function [125]. In our case, the probability density function of $P(t)$, denoted as $f_{1}(p, t)$, is characterized by the relation $\mathbb{P}[P(t) \in \mathcal{B}]=\int_{\mathcal{B}} f_{1}(p, t) \mathrm{d} p$, where $\mathcal{B} \subseteq \mathbb{R}$ is any Borel set. If the density function exists, we say that the random variable is absolutely continuous. This function allows computing all statistical moments of $P(t)$, in particular, the expectation $\mathbb{E}$ and the variance $\mathbb{V}$, and also confidence intervals. Thus, the probability density function allows quantifying the uncertainty associated to the response process $P(t)$.

In the autonomous case (when $A(t)$ does not depend on $t$, so it is just a random variable), the computation of the probability density function $f_{1}(p, t)$ has been 
addressed in [52, 51], as an application of the random variable transformation technique.

Only recently, in [42] the authors studied the non-autonomous case (3.1). The authors established conditions under which the probability density function of $P(t)$ can be approximated, in the spirit of other previous contributions such as [43, 12, 19]. In [42, the diffusion coefficient $A(t)$ is written as a Karhunen-Loève expansion [89]: $A(t, \omega)=\mu_{A}(t)+\sum_{j=1}^{\infty} \sqrt{\nu_{j}} \phi_{j}(t) \xi_{j}(\omega)$, where $\mu_{A}(t)=\mathbb{E}[A(t)],\left\{\nu_{j}\right\}_{j=1}^{\infty}$ and $\left\{\phi_{j}(t)\right\}_{j=1}^{\infty}$ are the set of eigenvalues and eigenfunctions associated to the covariance integral operator of $A(t)$, and $\left\{\xi_{j}\right\}_{j=1}^{\infty}$ is a sequence of uncorrelated random variables with zero expectation and unit variance. This process is truncated as $A_{N}(t, \omega)=\mu_{A}(t)+\sum_{j=1}^{N} \sqrt{\nu_{j}} \phi_{j}(t) \xi_{j}(\omega)$, which gives rise to a truncation of the solution stochastic process $P(t)$, say $P_{N}(t)$. The probability density function of $P_{N}(t), f_{1}^{N}(p, t)$, is computed via the random variable transformation technique [42, Th. 1], by assuming absolute continuity of $P_{0}, \xi_{1}, \xi_{2}, \ldots$ and statistical independence. The convergence of $f_{1}^{N}(p, t)$ to $f_{1}(p, t)$ is established by imposing a Lipschitz condition on the density function of $P_{0}, f_{P_{0}}$, on $(0,1)$, and certain growth condition on $A(t)$ : for each $t$, there exists $C_{t}>0$ such that $\mathbb{E}\left[\exp \left(2 \int_{t_{0}}^{t} A_{N}(s) \mathrm{d} s\right)\right] \leq C_{t}$, for all $N \geq 1$. This growth condition is satisfied if $A(t)$ is a Gaussian process, or if $\xi_{1}, \xi_{2}, \ldots$ have support in a common bounded interval, see [20, pp. 29-31], although for a Gaussian stochastic process $A(t)$ the exact probability density function of $P(t)$ is already known, so the applicability of [42] is restricted.

In this chapter, we tackle the approximation to $f_{1}(p, t)$ analogously to our recent contribution [17]. We consider a general sequence $\left\{A_{N}(t)\right\}_{N=1}^{\infty}$ that approximates $A(t)$ in the Hilbert space $\mathrm{L}^{2}\left(\left[t_{0}, T\right] \times \Omega\right)$ (mean square approximation/expansion of $A(t))$. This includes Karhunen-Loève expansions, certain random power series [125, p. 99], etc. We do not assume anything else on $A_{N}(t)$ (no absolute continuity, no growth condition...). For each approximating process $A_{N}(t)$, we construct an approximating process $P_{N}(t)$ for the solution stochastic process $P(t)$. By supposing absolute continuity only for $P_{0}$ and via a modified version of the random variable transformation technique, we are able to compute the probability density function of $P_{N}(t), f_{1}^{N}(p, t)$, which is expressed in terms of an expectation. By using probability theory tools, the convergence of $f_{1}^{N}(p, t)$ to $f_{1}(p, t)$ is established by only requiring $f_{P_{0}}$ to be almost everywhere (a.e.) continuous and bounded on $(0,1)$ (which is milder than being Lipschitz).

After the theoretical discussion of the chapter, we will perform numerical examples that highlight the improvement of our results compared with [42]. 


\subsection{Main results}

If $P_{0}(\omega) \in(0,1)$ almost surely (a.s.) and $A(t)$ has a.s. integrable sample paths on $\left[t_{0}, T\right]$, then the sample path solution to $(3.1)$ is given by

$$
P(t, \omega)=\frac{1}{1+\mathrm{e}^{\int_{t_{0}}^{t}-A(s, \omega) \mathrm{d} s}\left(-1+\frac{1}{P_{0}(\omega)}\right)},
$$

for almost every $\omega \in \Omega$, and every $t \in\left[t_{0}, T\right]$. Notice that $P(t, \omega) \in(0,1)$. Our goal is to compute its (first) probability density function $f_{1}(p, t)$.

For convenience of notation, we denote $K(t)=\int_{t_{0}}^{t} A(s) \mathrm{d} s$ and $Z(t)=\mathrm{e}^{-K(t)}>$ $0, t \in\left[t_{0}, T\right]$.

Proposition 3.1 If $P_{0}$ is absolutely continuous with density function $f_{P_{0}}$, and $A(t)$ is any stochastic process independent of $P_{0}$, then $P(t)$ is absolutely continuous for each $t \in\left[t_{0}, T\right]$, with density function given by

$$
f_{1}(p, t)=\mathbb{E}\left[f_{P_{0}}\left(\frac{Z(t) p}{1+p(-1+Z(t))}\right) \frac{Z(t)}{(1+p(-1+Z(t)))^{2}}\right] .
$$

Proof. Notice that, since $A$ and $P_{0}$ are independent, then $Z$ and $P_{0}$ are also independent. If we denote by $\mathbb{P}_{Z(t)}=\mathbb{P} \circ Z(t)^{-1}$ the probability law of $Z(t)$, $t \in\left[t_{0}, T\right]$, and $\mathcal{B} \subseteq \mathbb{R}$ is any Borel set then

$$
\begin{aligned}
\mathbb{P}[P(t) \in \mathcal{B}] & =\int_{\mathbb{R}} \mathbb{P}[P(t) \in \mathcal{B} \mid Z(t)=z] \mathbb{P}_{Z(t)}(\mathrm{d} z) \\
& =\int_{\mathbb{R}} \mathbb{P}\left[\frac{1}{1+Z(t)\left(-1+1 / P_{0}\right)} \in \mathcal{B} \mid Z(t)=z\right] \mathbb{P}_{Z(t)}(\mathrm{d} z) \\
& =\int_{\mathbb{R}} \mathbb{P}\left[\frac{1}{1+z\left(-1+1 / P_{0}\right)} \in \mathcal{B}\right] \mathbb{P}_{Z(t)}(\mathrm{d} z),
\end{aligned}
$$

where in the last equality we have used the independence between $Z(t)$ and $P_{0}$. Note that

$$
\mathbb{P}\left[\frac{1}{1+z\left(-1+1 / P_{0}\right)} \in \mathcal{B}\right]=\int_{\mathcal{B}} f_{\frac{1}{1+z\left(-1+1 / P_{0}\right)}}(b) \mathrm{d} b,
$$

where $f_{1 /\left(1+z\left(-1+1 / P_{0}\right)\right)}$ is the density function of $1 /\left(1+z\left(-1+1 / P_{0}\right)\right)$. By the random variable transformation technique,

$$
f_{\frac{1}{1+z\left(-1+1 / P_{0}\right)}}(b)=f_{P_{0}}\left(\frac{z b}{1+b(-1+z)}\right) \frac{z}{(1+b(-1+z))^{2}} .
$$


Notice that the absolute value in the Jacobian term $\frac{z}{(1+b(-1+z))^{2}}$ is omitted because $Z(t)>0$ a.s. As a consequence,

$$
\begin{aligned}
\mathbb{P}[P(t) \in \mathcal{B}] & =\int_{\mathbb{R}} \int_{\mathcal{B}} f_{P_{0}}\left(\frac{z b}{1+b(-1+z)}\right) \frac{z}{(1+b(-1+z))^{2}} \mathrm{~d} b \mathbb{P}_{Z(t)}(\mathrm{d} z) \\
& =\int_{\mathcal{B}} \int_{\mathbb{R}} f_{P_{0}}\left(\frac{z b}{1+b(-1+z)}\right) \frac{z}{(1+b(-1+z))^{2}} \mathbb{P}_{Z(t)}(\mathrm{d} z) \mathrm{d} b \\
& =\int_{\mathcal{B}} \mathbb{E}\left[f_{P_{0}}\left(\frac{Z(t) b}{1+b(-1+Z(t))}\right) \frac{Z(t)}{(1+b(-1+Z(t)))^{2}}\right] \mathrm{d} b,
\end{aligned}
$$

where in the second equality we have utilized Fubini's theorem, which is justified by the non-negativity of the integrand, and in the third equality we have used the definition of expectation. Finally, realize that 3.4 tells us that $P(t)$ is absolutely continuous, for each $t \in\left[t_{0}, T\right]$, with density function given by (3.3.

Suppose that $A(t)$ can be expanded in the Lebesgue space $\mathrm{L}^{2}\left(\left[t_{0}, T\right] \times \Omega\right)$, as a limit of a sequence of stochastic processes $\left\{A_{N}(t)\right\}_{N=1}^{\infty}$. This includes Karhunen-Loève expansions: $A(t, \omega)=\mu_{A}(t)+\sum_{j=1}^{\infty} \sqrt{\nu_{j}} \phi_{j}(t) \xi_{j}(\omega)$; certain random power series: $A(t, \omega)=\sum_{j=0}^{\infty} Y_{j}(\omega) t^{j}$; etc. In such a case, the expectation from $(3.3)$ cannot be explicitly computed, in general. To intuitively approximate (3.3), one may truncate $A(t)$ by using $A_{N}(t)$. This gives rise to an approximating stochastic process $P_{N}(t)$ of the solution $P(t)$ given by $(3.2)$, defined as

$$
P_{N}(t, \omega)=\frac{1}{1+\mathrm{e}^{\int_{t_{0}}^{t}-A_{N}(s, \omega) \mathrm{d} s}\left(-1+\frac{1}{P_{0}(\omega)}\right)} .
$$

Note that $P_{N}(t, \omega) \in(0,1)$. In practice, as $A_{N}$ may depend only on a finite number of random variables, the density function of $P_{N}(t)$ may be explicitly computed by using the following Proposition 3.2, which is completely analogous to Proposition 3.1. For example, in the case of Karhunen-Loève expansions, $A_{N}(t, \omega)=\mu_{A}(t)+\sum_{j=1}^{N} \sqrt{\nu_{j}} \phi_{j}(t) \xi_{j}(\omega)$; in the case of random power series, $A_{N}(t, \omega)=\sum_{j=0}^{N} Y_{j}(\omega) t^{j}$; etc., so that $A_{N}(t)$ only depends on finitely many random variables. 
For convenience of notation, we denote $K_{N}(t)=\int_{t_{0}}^{t} A_{N}(s) \mathrm{d} s$ and $Z_{N}(t)=$ $\mathrm{e}^{-K_{N}(t)}>0, t \in\left[t_{0}, T\right]$. The following fact will be essential later on:

$$
\begin{aligned}
\left\|K_{N}(t)-K(t)\right\|_{\mathrm{L}^{2}(\Omega)} & =\left(\mathbb{E}\left[\left(\int_{t_{0}}^{t}\left(A_{N}(s)-A(s)\right) \mathrm{d} s\right)^{2}\right]\right)^{\frac{1}{2}} \\
& \leq \sqrt{T-t_{0}}\left\|A_{N}-A\right\|_{\mathrm{L}^{2}\left(\left[t_{0}, T\right] \times \Omega\right)} \stackrel{N \rightarrow \infty}{\longrightarrow} 0
\end{aligned}
$$

for each $t \in\left[t_{0}, T\right]$, as a consequence of the well-known Cauchy-Schwarz inequality [125, p. 19].

Proposition 3.2 If $P_{0}$ is absolutely continuous with density function $f_{P_{0}}$, and $A_{N}(t)$ is independent of $P_{0}$, then $P_{N}(t)$ is absolutely continuous for each $t \in$ $\left[t_{0}, T\right]$, with density function given by

$$
f_{1}^{N}(p, t)=\mathbb{E}\left[f_{P_{0}}\left(\frac{Z_{N}(t) p}{1+p\left(-1+Z_{N}(t)\right)}\right) \frac{Z_{N}(t)}{\left(1+p\left(-1+Z_{N}(t)\right)\right)^{2}}\right] .
$$

In what follows, we demonstrate the convergence

$$
\lim _{N \rightarrow \infty} f_{1}^{N}(p, t)=f_{1}(p, t)
$$

for each $p \in(0,1)$ and $t \in\left[t_{0}, T\right]$ (recall that, for $p \notin(0,1)$, we have $f_{1}^{N}(p, t)=$ $\left.f_{1}(p, t)=0\right)$.

Theorem 3.3 Suppose that $P_{0}$ is an absolute continuous random variable with density function $f_{P_{0}}$, which is continuous and bounded on $(0,1)$. Let $\left\{A_{N}(t)\right\}_{N=1}^{\infty}$ be any sequence of stochastic processes, independent of $P_{0}$, that tends to $A(t)$ in $\mathrm{L}^{2}\left(\left[t_{0}, T\right] \times \Omega\right)$. Then $\lim _{N \rightarrow \infty} f_{1}^{N}(p, t)=f_{1}(p, t)$ for each $p \in(0,1)$ and $t \in\left[t_{0}, T\right]$.

Proof. As we want expression $(3.6)$ to tend to 3.3 as $N \rightarrow \infty$, it suffices to prove that

$$
\begin{array}{r}
f_{P_{0}}\left(\frac{Z_{N}(t) p}{1+p\left(-1+Z_{N}(t)\right)}\right) \frac{Z_{N}(t)}{\left(1+p\left(-1+Z_{N}(t)\right)\right)^{2}} \\
\stackrel{Z \rightarrow \infty}{\longrightarrow} f_{P_{0}}\left(\frac{Z(t) p}{1+p(-1+Z(t))}\right) \frac{Z(t)}{(1+p(-1+Z(t)))^{2}},
\end{array}
$$

in $\mathrm{L}^{1}(\Omega)$, see [125, Th. 4.2.1]. In the notation of [42, p. 128], let the functions

$$
g(z)=\frac{\mathrm{e}^{-z}}{\left(1+p\left(-1+\mathrm{e}^{-z}\right)\right)^{2}}, \quad h(z)=\frac{\mathrm{e}^{-z} p}{1+p\left(-1+\mathrm{e}^{-z}\right)}, \quad z \in \mathbb{R} .
$$


Then (3.7) may be rewritten as

$$
f_{P_{0}}\left(h\left(K_{N}(t)\right)\right) g\left(K_{N}(t)\right) \stackrel{N \rightarrow \infty}{\longrightarrow} f_{P_{0}}(h(K(t))) g(K(t)) \text { in } \mathrm{L}^{1}(\Omega) .
$$

By Cauchy-Schwarz inequality [125, p. 19], it suffices to establish the following limits:

$$
\begin{aligned}
f_{P_{0}}\left(h\left(K_{N}(t)\right)\right) & \stackrel{N \rightarrow \infty}{\longrightarrow} f_{P_{0}}(h(K(t))) \text { in } \mathrm{L}^{2}(\Omega), \\
g\left(K_{N}(t)\right) & \stackrel{N \rightarrow \infty}{\longrightarrow} g(K(t)) \text { in } \mathrm{L}^{2}(\Omega) .
\end{aligned}
$$

As it was proved in [42, p. 128], by using the deterministic mean value theorem, $\left|g\left(z_{1}\right)-g\left(z_{2}\right)\right| \leq C_{g}\left|z_{1}-z_{2}\right|$ and $\left|h\left(z_{1}\right)-h\left(z_{2}\right)\right| \leq C_{h}\left|z_{1}-z_{2}\right|$, for all $z_{1}, z_{2} \in \mathbb{R}$, for certain constants $C_{g}, C_{h}>0$. Then

$$
\begin{aligned}
& \left\|g\left(K_{N}(t)\right)-g(K(t))\right\|_{\mathrm{L}^{2}(\Omega)} \leq C_{g}\left\|K_{N}(t)-K(t)\right\|_{\mathrm{L}^{2}(\Omega)} \stackrel{N \rightarrow \infty}{\longrightarrow} 0, \\
& \left\|h\left(K_{N}(t)\right)-h(K(t))\right\|_{\mathrm{L}^{2}(\Omega)} \leq C_{h}\left\|K_{N}(t)-K(t)\right\|_{\mathrm{L}^{2}(\Omega)} \stackrel{N \rightarrow \infty}{\longrightarrow} 0,
\end{aligned}
$$

as a consequence of $(3.5)$. Thus, by the latter relation, 3.9 is proved. On the other hand, (3.8) is a consequence of the dominated convergence theorem [113, result 11.32 , p. 321]. Indeed, (3.8) is equivalent to proving that, for each subsequence $\left\{h\left(K_{N_{m}}(t)\right)\right\}_{m=1}^{\infty}$, there exists another subsequence $\left\{h\left(K_{N_{m_{l}}}(t)\right)\right\}_{l=1}^{\infty}$ such that $\lim _{l \rightarrow \infty} f_{P_{0}}\left(h\left(K_{N_{m_{l}}}(t)\right)\right)=f_{P_{0}}(h(K(t)))$ in $\mathrm{L}^{2}(\Omega)$, see [17, Th. 2.5]. Fix a subsequence $\left\{h\left(K_{N_{m}}(t)\right)\right\}_{m=1}^{\infty}$. As it converges to $h(K(t))$ in $\mathrm{L}^{2}(\Omega)$, by [11, Th. 4.9] there exists a subsequence $\left\{h\left(K_{N_{m_{l}}}(t)\right)\right\}_{l=1}^{\infty}$ that converges a.s. to $h(K(t))$. Since $f_{P_{0}}$ is continuous on $(0,1)$ and $h\left(K_{N_{m_{l}}}(t)\right), h(K(t)) \in(0,1)$, for all $l \geq 1$, we derive that $\lim _{l \rightarrow \infty} f_{P_{0}}\left(h\left(K_{N_{m_{l}}}(t)\right)\right)=f_{P_{0}}(h(K(t)))$ a.s. As $\left|f_{P_{0}}\left(h\left(K_{N_{m_{l}}}(t)\right)\right)\right| \leq\left\|f_{P_{0}}\right\|_{L^{\infty}(0,1)}$ a.s., for each $l \geq 1$, by the dominated convergence theorem we conclude that $\lim _{l \rightarrow \infty} f_{P_{0}}\left(h\left(K_{N_{m_{l}}}(t)\right)\right)=f_{P_{0}}(h(K(t)))$ in $\mathrm{L}^{2}(\Omega)$. Thus, (3.8) has been proved, and we are done.

In the following theorem, we allow a.e. continuity on $(0,1)$ for $f_{P_{0}}$. This is important, as we enlarge the applicability of our theoretical results to initial conditions $P_{0}$ such that $f_{P_{0}}$ has a jump discontinuity in $(0,1)$ (for instance, the uniform distribution, a truncated distribution with endpoint in $(0,1)$, etc.).

Theorem 3.4 Suppose that $P_{0}$ is an absolute continuous random variable with density function $f_{P_{0}}$, which is a.e. continuous and bounded on $(0,1)$. Let $\left\{A_{N}(t)\right\}_{N=1}^{\infty}$ be any sequence of stochastic processes, independent of $P_{0}$, that tends to $A(t)$ in $\mathrm{L}^{2}\left(\left[t_{0}, T\right] \times \Omega\right)$. Suppose that $\int_{t_{0}}^{t} A(t) \mathrm{d} s$ is absolutely continuous for each $t \in\left[t_{0}, T\right]$. Then $\lim _{N \rightarrow \infty} f_{1}^{N}(p, t)=f_{1}(p, t)$ for each $p \in(0,1)$ and $t \in\left[t_{0}, T\right]$. 
Proof. The proof is completely analogous to Theorem 3.3, see [17, Th. 2.7] for the original idea. The only step that needs to be justified is the limit $\lim _{l \rightarrow \infty} f_{P_{0}}\left(h\left(K_{N_{m_{l}}}(t)\right)\right)=f_{P_{0}}(h(K(t)))$ a.s. Since $\int_{t_{0}}^{t} A(t) \mathrm{d} s$ is absolutely continuous, then $h(K(t))$ is absolutely continuous, so $\mathbb{P}\left[h(K(t)) \in \mathcal{D}_{f_{P_{0}}}\right]=0$, where $\mathcal{D}_{f_{P_{0}}}$ is the null set of discontinuities of $f_{P_{0}}$. By the continuous mapping theorem [135, p. 7, Th. 2.3], the convergence $\lim _{l \rightarrow \infty} f_{P_{0}}\left(h\left(K_{N_{m_{l}}}(t)\right)\right)=$ $f_{P_{0}}(h(K(t)))$ a.s. is justified.

Notice that Theorem 3.3 and Theorem 3.4 extend [42], as [42] assumes that all data are absolutely continuous, the expansion of $A(t)$ is of Karhunen-Loèvetype with certain growth, and $f_{P_{0}}$ is Lipschitz on $(0,1)$ (which is a stronger assumption than continuity and boundedness on $(0,1)$, see [20, p. 10]).

Notice also that the hypothesis of absolute continuity for $\int_{t_{0}}^{t} A(t) \mathrm{d} s$ in Theorem 3.4 is easily satisfied in the case of Karhunen-Loève expansions and random power series. If $A(t, \omega)=\mu_{A}(t)+\sum_{j=1}^{\infty} \sqrt{\nu_{j}} \phi_{j}(t) \xi_{j}(\omega)$ or $A(t, \omega)=$ $\sum_{j=0}^{\infty} Y_{j}(\omega) t^{j}$, then it suffices that one of the $\xi_{j}$ 's or one of the $Y_{j}$ 's is absolutely continuous and independent of the rest of random coefficients, by [17, Lemma 2.1].

It is easy to prove that $P_{N}(t) \rightarrow P(t)$ as $N \rightarrow \infty$ in $\mathrm{L}^{2}(\Omega)$, for each $t \in$ $\left[t_{0}, T\right]$ (see Proposition 3.5), so that the expectation and the variance of $P(t)$ may be approximated as follows: $\mathbb{E}[P(t)]=\lim _{N \rightarrow \infty} \mathbb{E}\left[P_{N}(t)\right]$ and $\mathbb{V}[P(t)]=$ $\lim _{N \rightarrow \infty} \mathbb{V}\left[P_{N}(t)\right]$, where both statistics expectation $\mathbb{E}\left[P_{N}(t)\right]=\int_{\mathbb{R}} p f_{1}^{N}(p, t) \mathrm{d} p$ and variance $\mathbb{V}\left[P_{N}(t)\right]=\mathbb{E}\left[P_{N}(t)^{2}\right]-\left(\mathbb{E}\left[P_{N}(t)\right]\right)^{2}, \mathbb{E}\left[P_{N}(t)^{2}\right]=\int_{\mathbb{R}} p^{2} f_{1}^{N}(p, t) \mathrm{d} p$ can be computed with $f_{1}^{N}(p, t)$. This is a key property of mean square convergence, see [125, Th. 4.2.1, Th. 4.3.1].

Proposition 3.5 We have that $\lim _{N \rightarrow \infty} P_{N}(t)=P(t)$ in $\mathrm{L}^{2}(\Omega)$, for each $t \in$ $\left[t_{0}, T\right]$.

Proof. It suffices to prove that, for each subsequence $\left\{P_{N_{m}}(t)\right\}_{m=1}^{\infty}$, there exists another subsequence $\left\{P_{N_{m_{l}}}(t)\right\}_{l=1}^{\infty}$ such that $\lim _{l \rightarrow \infty} P_{N_{m_{l}}}(t)=P(t)$ in $\mathrm{L}^{2}(\Omega)$, for each $t \in\left[t_{0}, T\right]$. This is due to a property of convergence already used in Theorem 3.3 and [17, Th. 2.5]. Fix a subsequence $\left\{P_{N_{m}}(t)\right\}_{m=1}^{\infty}$. We know that $\lim _{m \rightarrow \infty} K_{N_{m}}(t)=K(t)$ in $\mathrm{L}^{2}(\Omega)$, by (3.5). By [11, Th. 4.9], there exists a subsequence $\left\{K_{N_{m_{l}}}(t)\right\}_{l=1}^{\infty}$ that converges to $K(t)$ a.s. This implies that $\left\{P_{N_{m_{l}}}(t)\right\}_{l=1}^{\infty}$ converges to $P(t)$ a.s. Since $\left|P_{N_{m_{l}}}(t)\right|<1$ a.s., for each $l \geq 1$, by the dominated convergence theorem we derive that $\lim _{l \rightarrow \infty} P_{N_{m_{l}}}(t)=P(t)$ in $L^{2}(\Omega)$. This concludes the proof. 


\subsection{Numerical examples}

In this section we illustrate our theoretical findings with four numerical examples in which we approximate the probability density function of $P(t), f_{1}(p, t)$, as well as its expectation and variance, $\mathbb{E}[P(t)]$ and $\mathbb{V}[P(t)]$, by means of the approximating density functions $f_{1}^{N}(p, t)$.

The computations are performed in the software Mathematica ${ }^{\circledR}$, version 11.2.

Example 3.6 deals with a type of problem (3.1) that satisfies the hypotheses of our theorems and also [42, so that our methodology and 42 provide the same conclusions. Example 3.7 does not satisfy assumption $\mathrm{H} 4$ from [42, but our theorems are applicable, so that we enlarge the applicability of [42]. Example 3.8 deals with discrete distributed random data, which can be done due to our modification of the random variable transformation technique from Proposition 3.1 and Proposition 3.2, in contrast with [42]. Finally, Example 3.9 deals with a diffusion coefficient expressed as a random power series, thus showing that we are not restricted to Karhunen-Loève expansions as in [42].

Example 3.6 Consider (3.1) on $\left[t_{0}, T\right]=[0,1]$, with $P_{0}$ having a truncated Exponential(5) distribution on $[0,1]$, and $A(t)$ expressed as a Karhunen-Loève expansion written as

$$
A(t)=\sum_{j=1}^{\infty} \frac{\sqrt{2}}{j \pi} \sin (t j \pi) \xi_{j},
$$

where each $\xi_{i}$ is expressed as $\xi_{i}=X_{i}-Y_{i}, i \geq 1$, where $X_{1}, Y_{1}, X_{2}, Y_{2}, \ldots$ are independent random variables distributed as Exponential $(\sqrt{2})$, and independent of $P_{0}$ too. Notice that $\xi_{1}, \xi_{2}, \ldots$ are independent, with $\mathbb{E}\left[\xi_{i}\right]=0$ and $\mathbb{V}\left[\xi_{i}\right]=1$, so that the Karhunen-Loève expansion of $A(t)$ is written appropriately. We consider the truncations $A_{N}(t)=\sum_{j=1}^{N} \frac{\sqrt{2}}{j \pi} \sin (t j \pi) \xi_{j}$, which converge in $\mathrm{L}^{2}([0,1] \times \Omega)$ to $A(t)$ as $N \rightarrow \infty$.

Our Theorem 3.3 is applicable. Notice also that the assumptions of 42 hold, as we are dealing with a diffusion coefficient $A(t)$ expressed as a KarhunenLoève expansion with absolutely continuous random coefficients $\xi_{1}, \xi_{2}, \ldots, f_{P_{0}}$ is Lipschitz on $(0,1)$, and $\mathrm{H} 4$ from [42, p. 129] holds. Indeed, by the independence and by the expression of the moment-generating function of an 
Exponential distribution, $\varphi(\cdot)$, we have:

$$
\begin{aligned}
& \mathbb{E}\left[\mathrm{e}^{2 K_{N}(t)}\right]=\mathbb{E}\left[\mathrm{e}^{2 \int_{0}^{t} A_{N}(s) \mathrm{d} s}\right]=\mathbb{E}\left[\mathrm{e}^{\sum_{j=1}^{N} \frac{2 \sqrt{2}}{j \pi} \int_{0}^{t} \sin (s j \pi) \mathrm{d} s \xi_{j}}\right]=\mathbb{E}\left[\mathrm{e}^{\sum_{j=1}^{N} \frac{2 \sqrt{2}}{j^{2} \pi^{2}}(1-\cos (t j \pi)) \xi_{j}}\right] \\
= & \prod_{j=1}^{N} \mathbb{E}\left[\mathrm{e}^{\frac{2 \sqrt{2}}{j^{2} \pi^{2}}(1-\cos (t j \pi)) \xi_{j}}\right] \leq \prod_{j=1}^{N} \mathbb{E}\left[\mathrm{e}^{\frac{4 \sqrt{2}}{j^{2} \pi^{2}}\left(X_{j}+Y_{j}\right)}\right]=\left(\prod_{j=1}^{N} \varphi_{\operatorname{Exp}(\sqrt{2})}\left(\frac{4 \sqrt{2}}{j^{2} \pi^{2}}\right)\right)^{2} \\
= & \left(\prod_{j=1}^{N} \frac{\sqrt{2}}{\sqrt{2}-\frac{4 \sqrt{2}}{j^{2} \pi^{2}}}\right)^{2}=\left(\prod_{j=1}^{N}\left(1-\frac{4}{j^{2} \pi^{2}}\right)\right)^{-2} \leq C,
\end{aligned}
$$

for all $N \geq 1$, for certain $C>0$, since $\prod_{j=1}^{\infty}\left(1-\frac{4}{j^{2} \pi^{2}}\right)$ converges because $\sum_{j=1}^{\infty} \frac{4}{j^{2} \pi^{2}}<\infty$. Thereby, the methodology from [42] is also applicable.

In Figure 3.1, we plot $f_{1}^{N}(p, t)$ for orders of truncation $N=1,2,3$ and for $t=0.2$ and $t=0.6$, with the built-in function NExpectation. We observe that the theoretical convergence agrees with the numerical results depicted. We also observe fast convergence to $f_{1}(p, t)$. These computations are completed with approximations for the expectation and the variance of $P(t)$ in Table 3.1 .

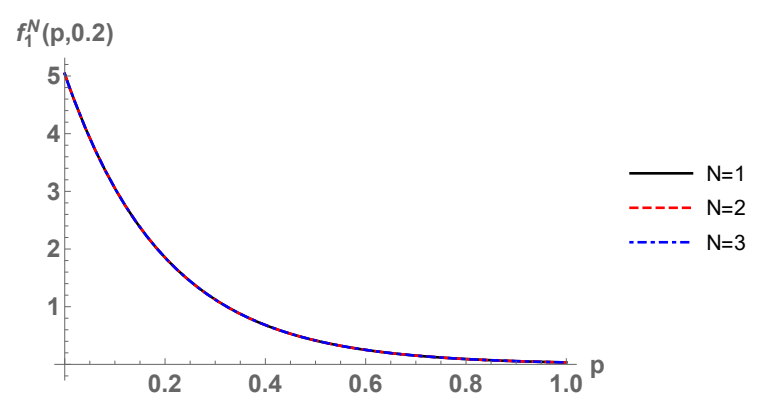

$f_{1}^{N}(p, 0.6)$

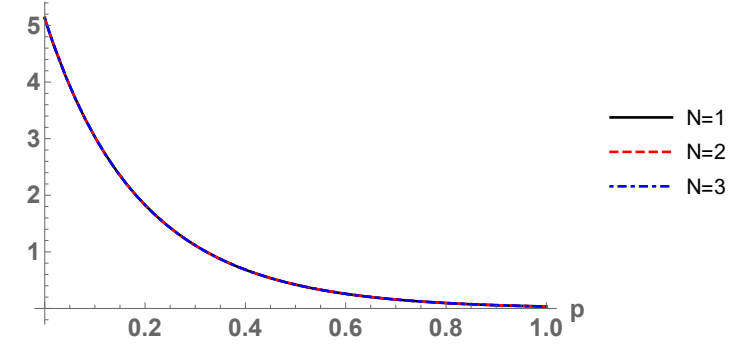

Figure 3.1: Plot of $f_{1}^{N}(p, t)$ for orders of truncation $N=1,2,3$ and for $t=0.2$ and $t=0.6$, in Example 3.6 


\begin{tabular}{|c|c|c|}
\hline & $t=0.2$ & $t=0.6$ \\
\hline $\mathbb{E}\left[P_{1}(t)\right]$ & 0.193236 & 0.194135 \\
\hline $\mathbb{E}\left[P_{2}(t)\right]$ & 0.193252 & 0.194245 \\
\hline $\mathbb{E}\left[P_{3}(t)\right]$ & 0.193264 & 0.194245 \\
\hline $\mathbb{V}\left[P_{1}(t)\right]$ & 0.0331839 & 0.0338054 \\
\hline $\mathbb{V}\left[P_{2}(t)\right]$ & 0.0331950 & 0.0338811 \\
\hline $\mathbb{V}\left[P_{3}(t)\right]$ & 0.0332029 & 0.0338813 \\
\hline
\end{tabular}

Table 3.1: Approximations $\mathbb{E}\left[P_{N}(t)\right]$ and $\mathbb{V}\left[P_{N}(t)\right]$ for $N=1,2,3$ and $t=0.2, t=0.6$, in Example 3.6

Example 3.7 In this example we address $(3.1)$ on $\left[t_{0}, T\right]=[0,1]$, where $P_{0} \sim \operatorname{Beta}(5,4)$ and $A(t)$ is expressed as the Karhunen-Loève expansion (3.10), where $\xi_{j}=X_{j} / \sqrt{3}$, being $X_{1}, X_{2}, \ldots$ random variables with Student's $t$ distribution with 3 degrees of freedom. It is assumed that $P_{0}, X_{1}, X_{2}, \ldots$ are statistically independent. Notice that $\mathbb{E}\left[\xi_{j}\right]=0$ and $\mathbb{V}\left[\xi_{j}\right]=1$, so that we have a proper Karhunen-Loève expansion for $A(t)$.

If we denote by $A_{N}(t)$ the $N$-th partial sum of the Karhunen-Loève series, it is easy to check that hypothesis $\mathrm{H} 4$ from [42] does not hold, because it is well-known that the Student's $t$-distribution does not possess a well-defined moment-generating function. Nonetheless, our new Theorem 3.3 is applicable, as it does not rely on any growth condition for the random coefficients $\xi_{1}, \xi_{2}, \ldots$

Let us perform some numerical experiments. In Figure 3.2, we plot the graph of $f_{1}^{N}(p, t)$ for orders $N=1,2,3$ at $t=0.2$ and $t=0.6$. With $f_{1}^{N}(p, t)$, we have computed $\mathbb{E}\left[P_{N}(t)\right]$ and $\mathbb{V}\left[P_{N}(t)\right]$ in Table 3.2 . The numerical results show fast convergence to the target density function $f_{1}(p, t)$, which corroborates our theoretical conclusions.

Example 3.8 We deal with $(3.1)$ on $\left[t_{0}, T\right]=[0,1]$, with $P_{0}$ that follows a truncated Exponential(3) distribution on $[0,1]$, and with $A(t)$ expressed by (3.10), where each $\xi_{j}$ follows a discrete uniform distribution on the set $\{-1,1\}$ that is, $\left.\mathbb{P}\left[\xi_{j}=-1\right]=\mathbb{P}\left[\xi_{j}=1\right]=1 / 2\right)$. We assume independence between $P_{0}, \xi_{1}, \xi_{2}, \ldots$..

In this case, as we have discrete random data, the methodology from [42] is not applicable. However, our Theorem 3.3 can certainly be used. 

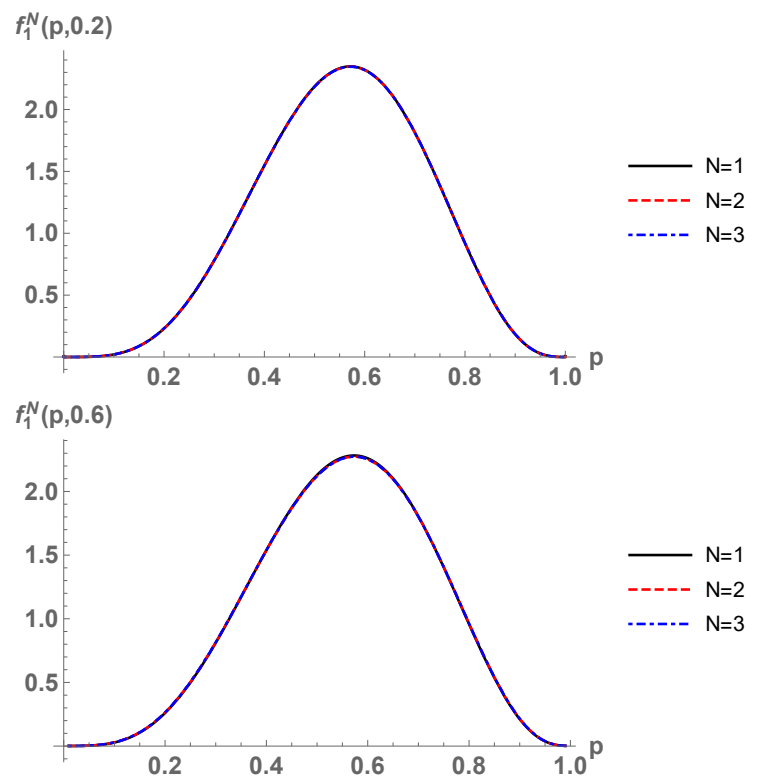

Figure 3.2: Plot of $f_{1}^{N}(p, t)$ for orders of truncation $N=1,2,3$ and for $t=0.2$ and $t=0.6$, in Example 3.7

\begin{tabular}{|c|c|c|}
\hline & $t=0.2$ & $t=0.6$ \\
\hline $\mathbb{E}\left[P_{1}(t)\right]$ & 0.555548 & 0.555242 \\
\hline $\mathbb{E}\left[P_{2}(t)\right]$ & 0.555542 & 0.555201 \\
\hline $\mathbb{E}\left[P_{3}(t)\right]$ & 0.555538 & 0.555180 \\
\hline $\mathbb{V}\left[P_{1}(t)\right]$ & 0.0247219 & 0.0259793 \\
\hline $\mathbb{V}\left[P_{2}(t)\right]$ & 0.0247469 & 0.0261431 \\
\hline $\mathbb{V}\left[P_{3}(t)\right]$ & 0.0247647 & 0.0262821 \\
\hline
\end{tabular}

Table 3.2: Approximations $\mathbb{E}\left[P_{N}(t)\right]$ and $\mathbb{V}\left[P_{N}(t)\right]$ for $N=1,2,3$ and $t=0.2, t=0.6$, in Example 3.7 
In Figure 3.3 , we show graphical representations of $f_{1}^{N}(p, t)$ for orders of truncation $N=1,2,3$ and for $t=0.2$ and $t=0.6$. Table 3.3 presents the approximations for $\mathbb{E}[P(t)]$ and $\mathbb{V}[P(t)]$. The computational results support the theoretical convergence.

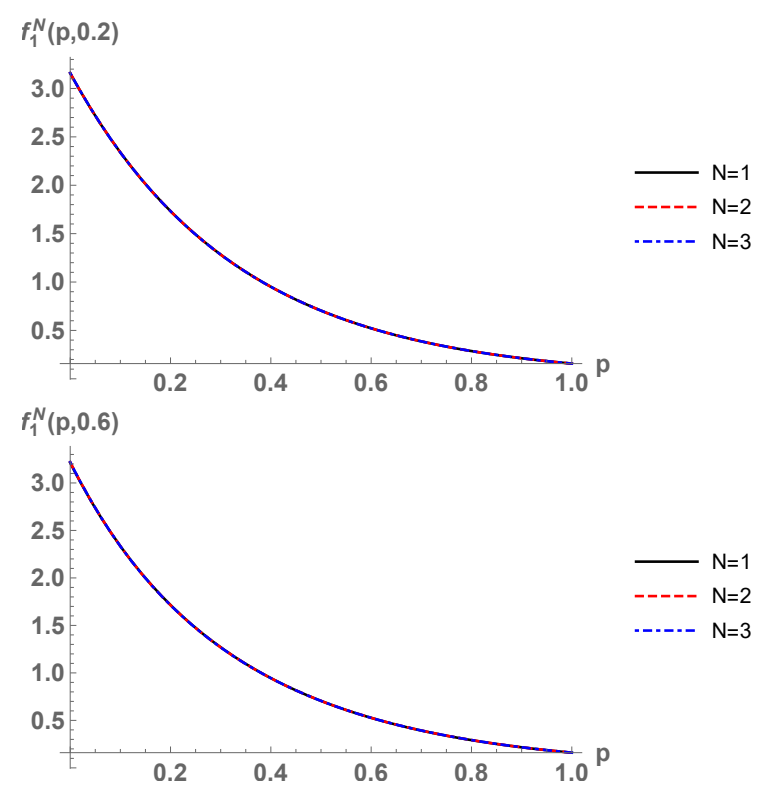

Figure 3.3: Plot of $f_{1}^{N}(p, t)$ for orders of truncation $N=1,2,3$ and for $t=0.2$ and $t=0.6$, in Example 3.8 .

\begin{tabular}{|c|c|c|}
\hline & $t=0.2$ & $t=0.6$ \\
\hline $\mathbb{E}\left[P_{1}(t)\right]$ & 0.280953 & 0.281662 \\
\hline $\mathbb{E}\left[P_{2}(t)\right]$ & 0.280966 & 0.281747 \\
\hline $\mathbb{E}\left[P_{3}(t)\right]$ & 0.280975 & 0.281748 \\
\hline $\mathbb{V}\left[P_{1}(t)\right]$ & 0.0559840 & 0.0566202 \\
\hline $\mathbb{V}\left[P_{2}(t)\right]$ & 0.0559953 & 0.0566972 \\
\hline $\mathbb{V}\left[P_{3}(t)\right]$ & 0.0560034 & 0.0566974 \\
\hline
\end{tabular}

Table 3.3: Approximations $\mathbb{E}\left[P_{N}(t)\right]$ and $\mathbb{V}\left[P_{N}(t)\right]$ for $N=1,2,3$ and $t=0.2, t=0.6$, in Example 3.8

Example 3.9 We consider $(3.1)$ on $\left[t_{0}, T\right]=[0,1], P_{0}$ having a $\operatorname{Normal}(2,1)$ distribution truncated on $[0.1,0.7]$, and with $A(t)$ expressed as a random power 
series on $\mathrm{L}^{2}([0,1] \times \Omega)$ :

$$
A(t)=\sum_{j=1}^{\infty} \frac{Y_{j}}{j^{3}} t^{j},
$$

where $Y_{1}, Y_{2}, \ldots$ are independent random variables with a Triangular distribution on $[2,3]$. We also suppose that $P_{0}$ is independent of $Y_{1}, Y_{2}, \ldots$. The partial sums $A_{N}(t)=\sum_{j=1}^{N} \frac{Y_{j}}{j^{3}} t^{j}$ form the approximating sequence to $A(t)$ in $\mathrm{L}^{2}([0,1] \times \Omega)$.

This example cannot be addressed with [42]. Our Theorem 3.4 is indeed applicable, as $f_{P_{0}}$ is a.e. continuous on $(0,1)$ (it only has two discontinuities on $(0,1)$, at $p=0.1$ and $p=0.7)$ and bounded. Figure 3.4 and Table 3.4 present the numerical results.

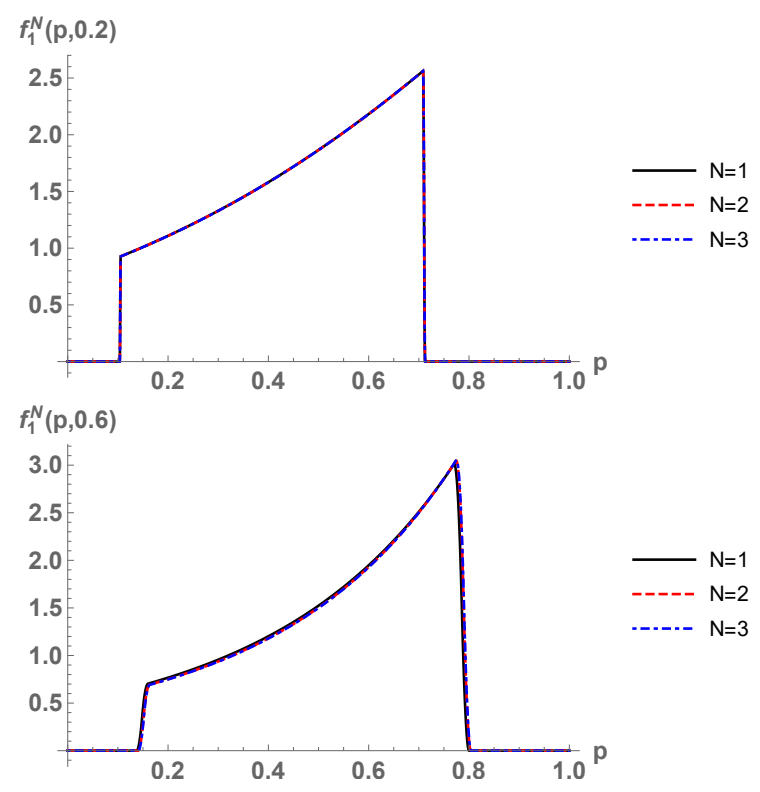

Figure 3.4: Plot of $f_{1}^{N}(p, t)$ for orders of truncation $N=1,2,3$ and for $t=0.2$ and $t=0.6$, in Example 3.9 


\begin{tabular}{|c|c|c|}
\hline & $t=0.2$ & $t=0.6$ \\
\hline $\mathbb{E}\left[P_{1}(t)\right]$ & 0.457686 & 0.545446 \\
\hline $\mathbb{E}\left[P_{2}(t)\right]$ & 0.457869 & 0.550332 \\
\hline $\mathbb{E}\left[P_{3}(t)\right]$ & 0.457877 & 0.550981 \\
\hline $\mathbb{V}\left[P_{1}(t)\right]$ & 0.0287887 & 0.0305547 \\
\hline $\mathbb{V}\left[P_{2}(t)\right]$ & 0.0287955 & 0.0305514 \\
\hline $\mathbb{V}\left[P_{3}(t)\right]$ & 0.0287958 & 0.0305504 \\
\hline
\end{tabular}

Table 3.4: Approximations $\mathbb{E}\left[P_{N}(t)\right]$ and $\mathbb{V}\left[P_{N}(t)\right]$ for $N=1,2,3$ and $t=0.2, t=0.6$, in Example 3.9

\subsection{Conclusions}

In this chapter, we have provided a comprehensive stochastic analysis of the random non-autonomous logistic differential equation. The diffusion coefficient $A(t)$ has been assumed to be a stochastic process and the initial condition $P_{0}$ has been taken as a random variable. The solution $P(t)$ thus becomes a stochastic process. The main goal has consisted in approximating the probability density function $f_{1}(p, t)$ of $P(t)$. We have fully extended the results of the recent contribution [42], which is the first one in dealing with the nonautonomous case of this important equation for growth modeling. The idea of our approach, which could be used for other random differential equations as we did in [17, could be summarized as follows: (i) by assuming absolute continuity only for the initial condition $P_{0}$, we have proved that $P(t)$ is absolutely continuous with density function $f_{1}(p, t)$ expressed in terms of an expectation, via a generalization of the random variable transformation technique to our particular setting; (ii) when $A(t)$ is expanded or approximated in terms of a sequence of stochastic processes $\left\{A_{N}(t)\right\}_{N=1}^{\infty}$ in $\mathrm{L}^{2}\left(\left[t_{0}, T\right] \times \Omega\right)$ (such as in Karhunen-Loève expansions, random power series, etc.), we have constructed a truncation $P_{N}(t)$ of $P(t)$, whose density function $f_{1}^{N}(p, t)$ is computable; (iii) finally, we have proved that $\lim _{N \rightarrow \infty} f_{1}^{N}(p, t)=f_{1}(p, t)$ pointwise by using powerful tools such as Cauchy-Schwarz inequality, dominated convergence theorem, continuous mapping theorem, etc., under the mild assumptions of a.e. continuity and boundedness of $f_{P_{0}}$. The numerical examples show that our theoretical discussion is widely applicable in practice and generalizes [42]. 


\section{Acknowledgements}

This work has been supported by the Spanish Ministerio de Economía y Competitividad grant MTM2017-89664-P.

The main results of this chapter have been published in [18]. 


\section{Chapter 4}

\section{Mathematical methods for the randomized non-autonomous Bertalanffy model}

In this chapter, the goal is to analyze the randomized non-autonomous Bertalanffy model: $x^{\prime}(t, \omega)=a(t, \omega) x(t, \omega)+b(t, \omega) x(t, \omega)^{\frac{2}{3}}, x\left(t_{0}, \omega\right)=$ $x_{0}(\omega)$, where $a(t, \omega)$ and $b(t, \omega)$ are stochastic processes and $x_{0}(\omega)$ is a random variable, all of them defined in an underlying complete probability space. Under some assumptions on $a, b$ and $x_{0}$, it is obtained a solution stochastic process, $x(t, \omega)$, both in the sample path and in the mean square senses. By using the Random Variable Transformation technique and Karhunen-Loève expansions, we construct a sequence of probability density functions that, under certain conditions, converges pointwise or uniformly to the density function of $x(t, \omega), f_{x(t)}(x)$. This permits approximating the expectation and the variance of $x(t, \omega)$. In the end, numerical experiments are carried out to put in practice our theoretical findings. 


\subsection{Introduction and motivation}

Bertalanffy model [7] is a biological ordinary differential equation model that describes the relationship between the metabolism and the growth of an organism. The metabolism is divided into anabolism (synthesis) and catabolism (destruction). The model assumes that the body weight $W(t)$, at the time instant $t$, of an animal is the result of the counteraction of the processes of anabolism and catabolism:

$$
W^{\prime}(t)=\eta W^{m}(t)-\kappa W^{n}(t),
$$

where $\eta$ and $\kappa$ are the constants of anabolism and catabolism, respectively, proportional to some power of the body weight at the time $t$. This model follows the law of allometry: the rate of change of weight depends on the constants of anabolism and catabolism via a power of the weight.

The surface rule states that the dependence of anabolism on body weight takes the power $m=2 / 3$ [139, 4] (however, other exponents have been suggested, see [111]). Bertalanffy justified, by using physiological facts and mathematical considerations, that the rate of catabolism should have the power $n=1$. Bertalanffy model thus becomes

$$
W^{\prime}(t)=\eta W^{\frac{2}{3}}(t)-\kappa W(t) .
$$

In this chapter, we want to perform a mathematical study of the randomized non-autonomous Bertalanffy model:

$$
\left\{\begin{array}{l}
x^{\prime}(t, \omega)=a(t, \omega) x(t, \omega)+b(t, \omega) x(t, \omega)^{\frac{2}{3}}, t \in\left[t_{0}, T\right], \omega \in \Omega \\
x\left(t_{0}, \omega\right)=x_{0}(\omega), \omega \in \Omega
\end{array}\right.
$$

In this setting, we are considering an underlying complete probability space $(\Omega, \mathcal{F}, \mathbb{P})$. The set $\Omega$ contains the outcomes, that will be denoted generically by $\omega$, the set $\mathcal{F}$ is the $\sigma$-algebra of events and $\mathbb{P}$ is the probability measure. In (4.1), we are also considering the stochastic processes

$$
a=\left\{a(t, \omega): t \in\left[t_{0}, T\right], \omega \in \Omega\right\}, \quad b=\left\{b(t, \omega): t \in\left[t_{0}, T\right], \omega \in \Omega\right\}
$$

and the random variable $x_{0}(\omega)$, all of them defined in the probability space $(\Omega, \mathcal{F}, \mathbb{P})$. The solution to the stochastic system, $x(t, \omega)$, becomes a stochastic process.

The mathematical term that encompasses equations such as (4.1) is random differential equation (RDE). The random autonomous Bertalanffy model has been studied, with applications to fish weight growth modeling, in [28]. The recent paper [20], that has become the main reference for this chapter, has 
studied the random non-autonomous inhomogeneous linear differential equation, by focusing on approximating the probability density function of the solution stochastic process. Its main contribution has been using KarhunenLoève expansions [89, Ch. 5] to construct the approximating sequence of density functions. Other mathematical studies of the Bertalanffy model, in the sense of stochastic differential equations (SDEs) of Itô type [106, have been carried out in [120, 110]. In SDEs of Itô type, the uncertainty is introduced in the deterministic differential equation by means of stochastic perturbations driven by the white noise process.

The goal of this chapter is to perform a mathematical study of the random non-autonomous Bertalanffy model (4.1). It is proved that, under certain conditions, there is a solution stochastic process in the sample path and the mean square senses. This is done by relating the random non-autonomous Bertalanffy model to the random non-autonomous linear differential equation from [20, via the usual change of variables performed in Bernoulli differential equations. The Random Variable Transformation technique permits relating the probability density functions of the solution processes to the random nonautonomous Bertalanffy model and the random non-autonomous linear differential equation. The Karhunen-Loève expansions of the coefficient stochastic processes in the RDE (4.1) allow us to obtain an approximating sequence of probability density functions for the density $f_{x(t)}(x)$ of the solution process $x(t, \omega)$. A numerical and computational treatment of the theoretical results obtained will be performed in the end.

\subsection{Solving the random non-autonomous Bertalanffy model}

There are different ways of interpreting the random differential equation 4.1. One way, which uses strongly the deterministic theory on differential equations, is the sample path interpretation [129, p. 2, SP problem], [114, p. 440]: fixed $\omega \in \Omega$, the random problem (4.1) becomes a deterministic problem, so one looks for stochastic processes $x(t, \omega)$ with absolutely continuous sample paths that solve (4.1). Another way consists in using $\mathrm{L}^{p}(\Omega)$ random calculus (see [125, Ch. 4] and [89, Section 5.5] for an introduction). We will study solutions to (4.1) in the mean square sense, according to the definition in [125, p. 118] or [129, p. 3, L $\mathrm{L}^{p}$ problem].

Consider the stochastic process

$$
x(t, \omega)=\left(x_{0}(\omega)^{\frac{1}{3}} \mathrm{e}^{\frac{1}{3} \int_{t_{0}}^{t} a(s, \omega) \mathrm{d} s}+\frac{1}{3} \int_{t_{0}}^{t} b(s, \omega) \mathrm{e}^{\frac{1}{3} \int_{s}^{t} a(r, \omega) \mathrm{d} r} \mathrm{~d} s\right)^{3} .
$$


This stochastic process comes from randomizing the deterministic solution to the deterministic non-autonomous Bertalanffy model. The integrals in 4.2 are considered in the Lebesgue sense, for each $\omega \in \Omega$ fixed (sample path Lebesgue integral), or in the mean square sense.

\subsubsection{Solution stochastic process with absolutely continuous sample paths}

By the classical theory on deterministic differential equations [68, p. 28-30], the following theorem holds:

\section{Theorem 4.1 (Sample path solution to the Bertalanffy model)}

Suppose that $a(\cdot, \omega), b(\cdot, \omega) \in \mathrm{L}^{1}\left(\left[t_{0}, T\right]\right)$, for a.e. $\omega \in \Omega$. Then the stochastic process $x(t, \omega)$ given by (4.2) satisfies that, for a.e. $\omega \in \Omega, x(\cdot, \omega)$ is absolutely continuous on $\left[t_{0}, T\right]$ and satisfies (4.1) for a.e. $t \in\left[t_{0}, T\right]$.

If $a(\cdot, \omega)$ and $b(\cdot, \omega)$ are continuous on $\left[t_{0}, T\right]$, then $x(\cdot, \omega)$ is in $C^{1}\left(\left[t_{0}, T\right]\right)$ and satisfies (4.1) for all $t \in\left[t_{0}, T\right]$.

\subsubsection{Solution stochastic process in the mean square sense}

Consider the random linear differential equation

$$
\left\{\begin{array}{l}
y^{\prime}(t, \omega)=\frac{1}{3} a(t, \omega) y(t, \omega)+\frac{1}{3} b(t, \omega), t \in\left[t_{0}, T\right], \omega \in \Omega, \\
y\left(t_{0}, \omega\right)=x_{0}(\omega)^{\frac{1}{3}}, \omega \in \Omega .
\end{array}\right.
$$

Notice that, if a stochastic process $y(t, \omega)$ is a solution to 4.3 in the $\mathrm{L}^{6}(\Omega)$ sense, then the stochastic process defined as $x(t, \omega)=y(t, \omega)^{3}$ is a solution to (4.1) in the $\mathrm{L}^{2}(\Omega)$ sense. Indeed, if $y(t, \omega)$ is differentiable in the $\mathrm{L}^{6}(\Omega)$ sense, then $x(t, \omega)$ is differentiable in the $\mathrm{L}^{2}(\Omega)$ sense, by [137, Lemma 3.14], and by the product rule, $x(t, \omega)$ solves $(4.1)$ in the $\mathrm{L}^{2}(\Omega)$ sense.

Let $y(t, \omega)$ be a stochastic process solution to 4.3$)$ in the $L^{6}(\Omega)$ sense. By [14, Th. 8-20] and [129, Th. 3(a)], there exists a stochastic process $\varphi(t, \omega)$ measurable on $\left[t_{0}, T\right] \times \Omega$, equivalent to $y(t, \omega)$ (meaning that $\varphi(t, \cdot)=y(t, \cdot)$ a.s., for all $\left.t \in\left[t_{0}, T\right]\right)$, such that $\varphi(\cdot, \omega)$ is absolutely continuous on $\left[t_{0}, T\right]$ and solves 4.3 in the sample path sense, for each $\omega \in \Omega$. By [20, Th. 1.3], up to equivalence,

$$
y(t, \omega)=x_{0}(\omega)^{\frac{1}{3}} \mathrm{e}^{\frac{1}{3} \int_{t_{0}}^{t} a(s, \omega) \mathrm{d} s}+\frac{1}{3} \int_{t_{0}}^{t} b(s, \omega) \mathrm{e}^{\frac{1}{3} \int_{s}^{t} a(r, \omega) \mathrm{d} r} \mathrm{~d} s
$$


Thus, 4.4 is the unique candidate, up to equivalence, that solves 4.3 in the $\mathrm{L}^{6}(\Omega)$ sense. We are going to show that, under some conditions on the stochastic processes $a(t, \omega)$ and $b(t, \omega)$, the stochastic process $y(t, \omega)$ given by (4.4) is indeed a solution to (4.3) in the $\mathrm{L}^{6}(\Omega)$ sense. From this fact, 4.2 will solve the random Bertalanffy model (4.1) in the mean square sense.

\section{Theorem 4.2 (Mean square solution to the Bertalanffy model)} Suppose that:

(i) $x_{0}, a$ and $b$ are independent.

(ii) $x_{0} \in \mathrm{L}^{4}(\Omega)$, and $a$ and $b$ are $\mathrm{L}^{12}(\Omega)$ continuous.

(iii) There exist $r>12$ and $\delta>0$ such that

$$
\sup _{s, s^{*} \in[-\delta, \delta]} \mathbb{E}\left[\mathrm{e}^{r \int_{x+s}^{t+s^{*}} a(u) \mathrm{d} u}\right]<\infty,
$$

for each $t_{0} \leq x \leq t \leq T$.

Then the stochastic process $y(t, \omega)$ defined by (4.4) (with mean square Riemann integrals) is differentiable in the $\mathrm{L}^{6}(\Omega)$ sense and satisfies the random problem (4.3). As a consequence, the stochastic process $x(t, \omega)$ defined by $(4.2)$ is differentiable in the mean square sense and satisfies the random Bertalanffy model (4.1).

Proof. The proof is straightforward from [39. Denote $y_{0}(\omega)=x_{0}(\omega)^{\frac{1}{3}}$, which belongs to $\mathrm{L}^{12}(\Omega)$. We apply the theory from [39, Section 3] adapted to $\mathrm{L}^{6}(\Omega)$ calculus.

Example 4.3 (Applications of Theorem 4.2) Let us see some examples of processes $a(t, \omega)$ for which the hypotheses of Theorem 4.2 fulfill.

- Let $\left[t_{0}, T\right]=[0,1]$ and $a(t, \omega)$ be a standard Brownian motion on $[0,1]$ [89, p. 185-186]. We have $a(t+h, \omega)-a(t, \omega) \sim \operatorname{Normal}(0, h)$, so

$$
\|a(t+h, \omega)-a(t, \omega)\|_{\mathrm{L}^{12}(\Omega)}=\|\sqrt{h} Z\|_{\mathrm{L}^{12}(\Omega)}=\sqrt{h}\|Z\|_{\mathrm{L}^{12}(\Omega)} \stackrel{h \rightarrow 0}{\longrightarrow} 0,
$$

being $Z \sim \operatorname{Normal}(0,1)$. This shows that $a(t, \omega)$ is continuous in the $\mathrm{L}^{12}(\Omega)$ sense. Another way of checking the $\mathrm{L}^{12}(\Omega)$-continuity of the Brownian motion uses [137, Lemma 3.11]: $a(t, \omega)$ is continuous in the $\mathrm{L}^{12}(\Omega)$ sense if and only if the function $\mathbb{E}\left[a\left(t_{1}, \omega\right) \cdots a\left(t_{12}, \omega\right)\right]$ defined on 
$\mathbb{R}^{12}$ is continuous on the diagonal $(t, \ldots, t) \in\left[t_{0}, T\right]^{12}$. This is clear by [125, p. 28].

On the other hand, since $a(t, \omega)$ is a Gaussian process, its mean square integral is normally distributed [125, Th. 4.6.4]. Bearing in mind the moment generating function of a normal distribution,

$$
\mathbb{E}\left[\mathrm{e}^{r \int_{x+s}^{t+s^{*}} a(u, \omega) \mathrm{d} u}\right]<\infty .
$$

- If $a(t, \omega)$ is a standard Brownian bridge on [0,1], [89, p. 193-195], the same holds, as $a(t, \omega)$ is a Gaussian process with stationary increments.

- An example of a non-Gaussian process is given by $a(t, \omega)=t U(\omega), t \in$ $\left[t_{0}, T\right]$, being $U$ any bounded random variable. Indeed, $a(t, \omega)$ is $\mathrm{L}^{12}(\Omega)$ continuous, since

$$
\|a(t+h, \omega)-a(t, \omega)\|_{\mathrm{L}^{12}(\Omega)}=|h|\|U\|_{\mathrm{L}^{12}(\Omega)} \stackrel{h \rightarrow 0}{\longrightarrow} 0 .
$$

On the other hand,

$\mathbb{E}\left[\mathrm{e}^{r \int_{x+s}^{t+s^{*}} a(u, \omega) \mathrm{d} u}\right] \leq \mathbb{E}\left[\mathrm{e}^{r\|U\|_{\mathrm{L}} \infty(\Omega) \int_{x+s}^{t+s^{*}} u \mathrm{~d} u}\right]=\mathrm{e}^{r\|U\|_{\mathrm{L} \infty(\Omega)} \frac{\left(t+s^{*}\right)^{2}-(x+s)^{2}}{2}}<\infty$,

so the hypotheses of Theorem 4.2 hold for $a(t, \omega)$.

\subsection{Obtaining the probability density function of the solution stochastic process}

The aim of this section is to approximate the probability density function of the solution stochastic process to the random Bertalanffy model, $x(t, \omega)$ given by (4.2). To achieve this goal, we will use existing results on the random linear differential equation from [20, together with a version of the Random Variable Transformation technique and the Karhunen-Loève expansions of both processes $a(t, \omega)$ and $b(t, \omega)$ from (4.1).

Lemma 4.4 (Random Variable Transformation method) ([83, p. 115]). Let $X$ be an absolutely continuous random variable with density $f_{X}$ and with support $D_{X}$ contained in an open set $D \subseteq \mathbb{R}$. Let $g: D \rightarrow \mathbb{R}$ be such that $D=\cup_{i=1}^{n} D_{i}$ and $g_{i}=\left.g\right|_{D_{i}}$ is injective and $C^{1}\left(D_{i}\right)$ with non-vanishing derivative. Then the random variable $Y=g(X)$ is absolutely continuous, with den- 
sity function given by

$$
f_{Y}(y)= \begin{cases}\sum_{i: y \in g\left(D_{i}\right)} f_{X}\left(g_{i}^{-1}(y)\right)\left|\frac{\mathrm{d} g_{i}^{-1}(y)}{\mathrm{d} y}\right|, & y \in g(D), \\ 0, & y \notin g(D) .\end{cases}
$$

Lemma 4.5 (Karhunen-Loève Theorem) ([89, Th. 5.28]). Consider a stochastic process $\{X(t, \omega): t \in \mathcal{T}, \omega \in \Omega\}$ in $\mathrm{L}^{2}(\mathcal{T} \times \Omega)$. Then

$$
X(t, \omega)=\mu(t)+\sum_{j=1}^{\infty} \sqrt{\nu_{j}} \phi_{j}(t) \xi_{j}(\omega)
$$

where the sum converges in $\mathrm{L}^{2}(\mathcal{T} \times \Omega), \mu(t)=\mathbb{E}[X(t)],\left\{\phi_{j}\right\}_{j=1}^{\infty}$ is an orthonormal basis of $\mathrm{L}^{2}(\mathcal{T}),\left\{\left(\nu_{j}, \phi_{j}\right)\right\}_{j=1}^{\infty}$ is the set of pairs of (nonnegative) eigenvalues and eigenvectors of the operator

$$
\mathcal{C}: \mathrm{L}^{2}(\mathcal{T}) \rightarrow \mathrm{L}^{2}(\mathcal{T}), \quad \mathcal{C} f(t)=\int_{\mathcal{T}} \mathbb{C o v}[X(t), X(s)] f(s) \mathrm{d} s,
$$

and $\left\{\xi_{j}\right\}_{j=1}^{\infty}$ is a sequence of random variables with zero expectation, unit variance and pairwise uncorrelated. In (4.6), Cov $[\cdot, \cdot]$ stands for the covariance operator. Moreover, if $\{X(t): t \in \mathcal{T}\}$ is a Gaussian process, then $\left\{\xi_{j}\right\}_{j=1}^{\infty}$ are independent and Gaussian.

\subsubsection{Main results}

Let $a(t, \omega)$ and $b(t, \omega)$ be stochastic processes in $\mathrm{L}^{2}\left(\left[t_{0}, T\right] \times \Omega\right)$. According to Lemma 4.5, we can expand both $a(t, \omega)$ and $b(t, \omega)$ via a Karhunen-Loève expansion:

$$
a(t, \omega)=\mu_{a}(t)+\sum_{j=1}^{\infty} \sqrt{\nu_{j}} \phi_{j}(t) \xi_{j}(\omega), \quad b(t, \omega)=\mu_{b}(t)+\sum_{j=1}^{\infty} \sqrt{\gamma_{j}} \psi_{j}(t) \eta_{j}(\omega)
$$

respectively. The summation symbol in both expansions will be always written up to $\infty$, although it could be possible that their corresponding covariance integral operators $\mathcal{C}$ have only a finite number of nonzero eigenvalues, so that the summation symbol finishes at an index $J<\infty$. As the more complex case arises when the sum arrives at infinity, we will always write the KarhunenLoève expansions of $a(t, \omega)$ and $b(t, \omega)$ up to infinity.

Notice that, from $a, b \in \mathrm{L}^{2}\left(\left[t_{0}, T\right] \times \Omega\right)$, we have $a(\cdot, \omega), b(\cdot, \omega) \in \mathrm{L}^{1}\left(\left[t_{0}, T\right]\right)$, therefore the process $x(t, \omega)$ has absolutely continuous sample paths and solves 
the random Bertalanffy model (4.1), by Theorem 4.1. Under the stricter assumptions of Theorem 4.2, the process $x(t, \omega)$ will be a mean square solution too.

For convenience, let us consider the truncation of the Karhunen-Loève expansions (4.7) of the stochastic processes $a$ and $b$ of common order $N$

$a_{N}(t, \omega)=\mu_{a}(t)+\sum_{j=1}^{N} \sqrt{\nu_{j}} \phi_{j}(t) \xi_{j}(\omega), \quad b_{N}(t, \omega)=\mu_{b}(t)+\sum_{j=1}^{N} \sqrt{\gamma_{j}} \psi_{j}(t) \eta_{j}(\omega)$.

This gives a truncation of the solution stochastic process $x(t, \omega)$ given by 4.2 :

$$
x_{N}(t, \omega)=\left(x_{0}(\omega)^{\frac{1}{3}} \mathrm{e}^{\frac{1}{3} \int_{t_{0}}^{t} a_{N}(s, \omega) \mathrm{d} s}+\frac{1}{3} \int_{t_{0}}^{t} b_{N}(s, \omega) \mathrm{e}^{\frac{1}{3} \int_{s}^{t} a_{N}(r, \omega) \mathrm{d} r} \mathrm{~d} s\right)^{3},
$$

We also obtain a truncation of the solution stochastic process $y(t, \omega)$ to the random linear differential equation, given by (4.4):

$$
y_{N}(t, \omega)=x_{0}(\omega)^{\frac{1}{3}} \mathrm{e}^{\frac{1}{3} \int_{t_{0}}^{t} a_{N}(s, \omega) \mathrm{d} s}+\frac{1}{3} \int_{t_{0}}^{t} b_{N}(s, \omega) \mathrm{e}^{\frac{1}{3} \int_{s}^{t} a_{N}(r, \omega) \mathrm{d} r} \mathrm{~d} s .
$$

The relation between both truncations (4.8) and (4.9) is that $x_{N}(t, \omega)=$ $y_{N}(t, \omega)^{3}$.

We denote, exactly as in [20], the following vectors in bold letters, $\boldsymbol{\xi}_{N}=$ $\left(\xi_{1}, \ldots, \xi_{N}\right)$ and $\boldsymbol{\eta}_{M}=\left(\eta_{1}, \ldots, \eta_{M}\right)$, understanding this as a random vector or as a deterministic real vector, depending on the context. Denote

$$
\begin{gathered}
K_{a}\left(t, \boldsymbol{\xi}_{N}\right)=\int_{t_{0}}^{t}\left(\mu_{a}(s)+\sum_{j=1}^{N} \sqrt{\nu_{j}} \phi_{j}(s) \xi_{j}\right) \mathrm{d} s, \\
S_{b}\left(s, \boldsymbol{\eta}_{N}\right)=\mu_{b}(s)+\sum_{i=1}^{N} \sqrt{\gamma_{i}} \psi_{i}(s) \eta_{i} .
\end{gathered}
$$

Suppose that $x_{0}$ and $\left(\xi_{1}, \ldots, \xi_{N}, \eta_{1}, \ldots, \eta_{N}\right)$ are absolutely continuous and independent, for each $N \geq 1$.

Let $y_{0}(\omega)=x_{0}(\omega)^{1 / 3}$ be the initial condition of the random linear differential equation (4.3). By Lemma 4.4 applied with the transformation mapping $g(x)=x^{1 / 3}$ on $D=\mathbb{R} \backslash\{0\}$, with domain partition $D=D_{1} \cup D_{2}$, being $D_{1}=(0, \infty)$ and $D_{2}=(-\infty, 0)$, we have $y_{0}(\omega)$ is absolutely continuous, with density function

for $y \in \mathbb{R}$.

$$
f_{y_{0}}(y)=f_{x_{0}}\left(y^{3}\right) 3 y^{2}
$$


By using the version of the Random Variable Transformation technique of [20, Lemma 2.1], in [20, Expression (10)] it was obtained the probability density function of $y_{N}(t, \omega)$ :

$$
\begin{aligned}
f_{y_{N}(t)}(y) & =\int_{\mathbb{R}^{2 N}} f_{y_{0}}\left(y \mathrm{e}^{-\frac{1}{3} K_{a}\left(t, \boldsymbol{\xi}_{N}\right)}-\frac{1}{3} \int_{t_{0}}^{t} S_{b}\left(s, \boldsymbol{\eta}_{N}\right) \mathrm{e}^{-\frac{1}{3} K_{a}\left(s, \boldsymbol{\xi}_{N}\right)} \mathrm{d} s\right) \\
& \times \mathrm{e}^{-\frac{1}{3} K_{a}\left(t, \boldsymbol{\xi}_{N}\right)} f_{\boldsymbol{\xi}_{N}, \boldsymbol{\eta}_{N}}\left(\boldsymbol{\xi}_{N}, \boldsymbol{\eta}_{N}\right) \mathrm{d} \boldsymbol{\xi}_{N} \mathrm{~d} \boldsymbol{\eta}_{N} \\
& =\mathbb{E}\left[f_{y_{0}}\left(y \mathrm{e}^{-\frac{1}{3} K_{a}\left(t, \boldsymbol{\xi}_{N}\right)}-\frac{1}{3} \int_{t_{0}}^{t} S_{b}\left(s, \boldsymbol{\eta}_{N}\right) \mathrm{e}^{-\frac{1}{3} K_{a}\left(s, \boldsymbol{\xi}_{N}\right)} \mathrm{d} s\right) \mathrm{e}^{-\frac{1}{3} K_{a}\left(t, \boldsymbol{\xi}_{N}\right)}\right] \\
& =\mathbb{E}\left[f_{x_{0}}\left(\left\{y \mathrm{e}^{-\frac{1}{3} K_{a}\left(t, \boldsymbol{\xi}_{N}\right)}-\frac{1}{3} \int_{t_{0}}^{t} S_{b}\left(s, \boldsymbol{\eta}_{N}\right) \mathrm{e}^{-\frac{1}{3} K_{a}\left(s, \boldsymbol{\xi}_{N}\right)} \mathrm{d} s\right\}^{3}\right)\right. \\
& \left.\times 3\left\{y \mathrm{e}^{-\frac{1}{3} K_{a}\left(t, \boldsymbol{\xi}_{N}\right)}-\frac{1}{3} \int_{t_{0}}^{t} S_{b}\left(s, \boldsymbol{\eta}_{N}\right) \mathrm{e}^{-\frac{1}{3} K_{a}\left(s, \boldsymbol{\xi}_{N}\right)} \mathrm{d} s\right\}^{2} \mathrm{e}^{-\frac{1}{3} K_{a}\left(t, \boldsymbol{\xi}_{N}\right)}\right],
\end{aligned}
$$

for $y \in \mathbb{R}$.

Since $x_{N}(t, \omega)=y_{N}(t, \omega)^{3}$, by Lemma 4.4 with the transformation mapping $g(x)=x^{3}$ on $D=\mathbb{R} \backslash\{0\}$, with domain partition $D=D_{1} \cup D_{2}$, being $D_{1}=$ $(0, \infty)$ and $D_{2}=(-\infty, 0)$, we have that $x_{N}(t, \omega)$ is an absolutely continuous random variable for each $t \in\left[t_{0}, T\right]$, with density function

$$
\begin{aligned}
f_{x_{N}(t)}(x) & =f_{y_{N}(t)}\left(x^{\frac{1}{3}}\right) \frac{1}{3 x^{\frac{2}{3}}} \\
& =\frac{1}{x^{\frac{2}{3}}} \mathbb{E}\left[f_{x_{0}}\left(\left\{x^{\frac{1}{3}} \mathrm{e}^{-\frac{1}{3} K_{a}\left(t, \boldsymbol{\xi}_{N}\right)}-\frac{1}{3} \int_{t_{0}}^{t} S_{b}\left(s, \boldsymbol{\eta}_{N}\right) \mathrm{e}^{-\frac{1}{3} K_{a}\left(s, \boldsymbol{\xi}_{N}\right)} \mathrm{d} s\right\}^{3}\right)\right. \\
& \left.\times\left\{x^{\frac{1}{3}} \mathrm{e}^{-\frac{1}{3} K_{a}\left(t, \boldsymbol{\xi}_{N}\right)}-\frac{1}{3} \int_{t_{0}}^{t} S_{b}\left(s, \boldsymbol{\eta}_{N}\right) \mathrm{e}^{-\frac{1}{3} K_{a}\left(s, \boldsymbol{\xi}_{N}\right)} \mathrm{d} s\right\}^{2} \mathrm{e}^{-\frac{1}{3} K_{a}\left(t, \boldsymbol{\xi}_{N}\right)}\right],
\end{aligned}
$$

for $0 \neq x \in \mathbb{R}$. Density functions are defined up to sets of Lebesgue measure 0 , so the fact that $f_{x_{N}(t)}(x)$ is not defined at $x=0$ is not any problem.

Under some assumptions, for instance, by taking into account [20, Th. 2.9, Th. 2.12], the random variable $y(t, \omega)$ is absolutely continuous for each $t \in$ $\left[t_{0}, T\right]$, with density function

$$
f_{y(t)}(y)=\lim _{N \rightarrow \infty} f_{y_{N}(t)}(y)
$$

for all $y \in \mathbb{R}$. Bearing in mind that $x(t, \omega)=y(t, \omega)^{3}$, by Lemma 4.4 with the transformation mapping $g(x)=x^{3}$ on $D=\mathbb{R} \backslash\{0\}$, we get that $x(t, \omega)$ is an absolutely continuous random variable, for each $t \in\left[t_{0}, T\right]$, with density 
function

$$
f_{x(t)}(x)=f_{y(t)}\left(x^{\frac{1}{3}}\right) \frac{1}{3 x^{\frac{2}{3}}},
$$

for $0 \neq x \in \mathbb{R}$. By combining 4.11, 4.13 and 4.14,

$$
f_{x(t)}(x)=f_{y(t)}\left(x^{\frac{1}{3}}\right) \frac{1}{3 x^{\frac{2}{3}}}=\lim _{N \rightarrow \infty} f_{y_{N}(t)}\left(x^{\frac{1}{3}}\right) \frac{1}{3 x^{\frac{2}{3}}}=\lim _{N \rightarrow \infty} f_{x_{N}(t)}(x),
$$

for all $0 \neq x \in \mathbb{R}$.

The goal is to find out under which conditions on the stochastic processes $a(t, \omega)$ and $b(t, \omega)$ and on the random variable $x_{0}(\omega)$ from (4.1), the solution stochastic process $x(t, \omega)$ given by (4.2) is an absolutely continuous random variable, for each $t \in\left[t_{0}, T\right]$, with density function satisfying

$$
f_{x(t)}(x)=\lim _{N \rightarrow \infty} f_{x_{N}(t)}(x),
$$

for each $0 \neq x \in \mathbb{R}$. For this purpose, we will use results on the random linear differential equation (4.3) [20, Th. 2.9, Th. 2.12], that establish under which conditions the limit 4.13 is justified.

Theorem 4.6 Assume the following four hypotheses:

$H 1: a, b \in \mathrm{L}^{2}\left(\left[t_{0}, T\right] \times \Omega\right)$;

H2: $x_{0}$ and $\left(\xi_{1}, \ldots, \xi_{N}, \eta_{1}, \ldots, \eta_{N}\right)$ are absolutely continuous and independent, $N \geq 1$

H3: the density function of $x_{0}, f_{x_{0}}$, is continuous on $\mathbb{R}$ and

$$
\begin{gathered}
f_{x_{0}}(x) \leq \frac{C}{|x|^{\frac{2}{3}}} \text {, for } x \neq 0 ; \\
H_{4}:\left\|\mathrm{e}^{-\frac{1}{3} K_{a}\left(t, \boldsymbol{\xi}_{N}\right)}\right\|_{\mathrm{L}^{2}(\Omega)} \leq C, \text { for all } N \geq 1 \text { and } t \in\left[t_{0}, T\right] .
\end{gathered}
$$

Then, for all $0 \neq x \in \mathbb{R}$ and $t \in\left[t_{0}, T\right]$, the sequence $\left\{f_{x_{N}(t)}(x)\right\}_{N=1}^{\infty}$ given by 4.12) converges to the density $f_{x(t)}(x)$ of the solution process $x(t, \omega)$ given by (4.2).

Proof. Since $y_{0}(\omega)=x_{0}(\omega)^{1 / 3}$, by hypothesis H2 $y_{0}$ and $\left(\xi_{1}, \ldots, \xi_{N}, \eta_{1}, \ldots, \eta_{N}\right)$ are absolutely continuous and independent, for $N \geq 1$. By 4.10 and hypothesis $\mathrm{H} 3, f_{y_{0}}(y)$ is continuous on $\mathbb{R}$ and

$$
f_{y_{0}}(y) \leq \frac{C}{\left|y^{3}\right|^{\frac{2}{3}}} 3 y^{2}=3 C,
$$


for $y \neq 0$, therefore bounded. The hypotheses of [20, Th. 2.9] are fulfilled for (4.3), therefore $y(t, \omega)$ is an absolutely continuous random variable for each $t \in\left[t_{0}, T\right]$, with density function satisfying (4.13). Then, $x(t, \omega)$ is absolutely continuous and verifies (4.15).

Theorem 4.7 Assume that

$H 1: a, b \in \mathrm{L}^{2}\left(\left[t_{0}, T\right] \times \Omega\right)$;

H2 : $x_{0}, \eta_{1},\left(\xi_{1}, \ldots, \xi_{N}, \eta_{2}, \ldots, \eta_{N}\right)$ are absolutely continuous and independent, $N \geq 1$;

H3: the density function of $\eta_{1}, f_{\eta_{1}}$, is continuous and bounded on $\mathbb{R}$;

$H_{4}: \xi_{1}, \xi_{2}, \ldots$ have compact support in $[-A, A](A>0), \psi_{1}>0$ on $\left(t_{0}, T\right)$.

Then, for each $0 \neq x \in \mathbb{R}$ and $t \in\left(t_{0}, T\right]$, the sequence $\left\{f_{x_{N}(t)}(x)\right\}_{N=1}^{\infty}$ given by (4.12) converges to the density $f_{x(t)}(x)$ of the solution process $x(t, \omega)$ given by (4.2).

Proof. By [20, Th. 2.12], $y(t, \omega)$ is an absolutely continuous random variable for each $t \in\left[t_{0}, T\right]$, with density function satisfying $(4.13)$. Then, $x(t, \omega)$ is absolutely continuous and verifies the desired limit 4.15).

By using the following lemma, we will establish a theorem similar to Theorem 4.6, but which substitutes the continuity hypothesis in H3 by a.e. continuity. This is important, as in H3 we will allow densities with some discontinuities in $\mathbb{R}$, such as the uniform distribution, exponential distribution, etc.

Lemma 4.8 Let $U$ and $V$ be two independent random variables. If $U$ is absolutely continuous, then $U+V$ is absolutely continuous.

Proof. For any Borel set $A$, by the convolution formula [8, p. 266] we have $\mathbb{P}(U+V \in A)=\int_{\mathbb{R}} \mathbb{P}(U \in A-v) \mathbb{P}_{V}(\mathrm{~d} v)$, where $\mathbb{P}_{V}=\mathbb{P} \circ V^{-1}$ is the law of $V$. If $A$ is null, then $A-v$ is null, so $\mathbb{P}(U \in A-v)=0$. Thus, if $A$ is null, then $\mathbb{P}(U+V \in A)=0$. By the Radon-Nikodym Theorem [147, Ch. 14], $U+V$ has a density. 
Theorem 4.9 Assume the following five hypotheses:

$H 1: a, b \in \mathrm{L}^{2}\left(\left[t_{0}, T\right] \times \Omega\right)$;

H2: $x_{0}, \xi_{1}, \ldots, \xi_{N}, \eta_{1}, \ldots, \eta_{N}$ are absolutely continuous and independent, $N \geq 1$

H3: the density function of $x_{0}, f_{x_{0}}$, is a.e. continuous on $\mathbb{R}$ and

$$
f_{x_{0}}(x) \leq \frac{C}{|x|^{\frac{2}{3}}}, \text { for a.e. } x \neq 0
$$

$H_{4}:\left\|\mathrm{e}^{-\frac{1}{3} K_{a}\left(t, \boldsymbol{\xi}_{N}\right)}\right\|_{\mathrm{L}^{2}(\Omega)} \leq C$, for all $N \geq 1$ and $t \in\left[t_{0}, T\right]$;

H5: $\psi_{1}(t) \neq 0$ for all $t \in\left(t_{0}, T\right)$.

Then, for all $0 \neq x \in \mathbb{R}$ and $t \in\left[t_{0}, T\right]$, the sequence $\left\{f_{x_{N}(t)}(x)\right\}_{N=1}^{\infty}$ given by (4.12) converges to the density $f_{x(t)}(x)$ of the solution process $x(t, \omega)$ given by 4.2.).

Proof. The proof is analogous to Theorem 4.6, but with a slight modification. We analyze the proof of [20, Th. 2.9]. In the notation of [20, Th. 2.9], $x Y-Z$ is absolutely continuous, by Lemma 4.8, $\mathrm{H} 2$ and $\mathrm{H} 5$. Then the probability that $x Y-Z$ belongs to the discontinuity set of $f_{x_{0}}$ is 0 . Recalling that in [20, Th. 2.9] one has $x Y_{N}(\omega)-Z_{N}(\omega) \rightarrow x Y(\omega)-Z(\omega)$ a.s. as $N \rightarrow \infty$, by the Continuous Mapping Theorem [135, p. 7, Th. 2.3] it follows $\mid f_{0}\left(x Y_{N}(\omega)-\right.$ $\left.Z_{N}(\omega)\right)-\left.f_{0}(x Y(\omega)-Z(\omega))\right|^{2} \rightarrow 0$ a.s. as $N \rightarrow \infty$. With this fact, the proof of [20, Th. 2.9] is applicable, as we did in Theorem 4.6.

Finally, we can establish results on uniform convergence of $\left\{f_{x_{N}(t)}(x)\right\}_{N=1}^{\infty}$, as a consequence of [20, Th. 2.4, Th. 2.7].

Theorem 4.10 Assume the following four hypotheses:

$H 1: a, b \in \mathrm{L}^{2}\left(\left[t_{0}, T\right] \times \Omega\right) ;$

H2: $x_{0}$ and $\left(\xi_{1}, \ldots, \xi_{N}, \eta_{1}, \ldots, \eta_{N}\right)$ are absolutely continuous and independent, $N \geq 1$

H3: the function $f_{x_{0}}\left(x^{3}\right) x^{2}$ is Lipschitz on $\mathbb{R}$;

$H_{4}$ : there exist $2 \leq p \leq \infty$ and $4 \leq q \leq \infty$ such that $1 / p+2 / q=1 / 2$,

$$
\begin{aligned}
& \left\|\mu_{b}\right\|_{\mathrm{L}^{p}\left(t_{0}, T\right)}+\sum_{j=1}^{\infty} \sqrt{\gamma_{j}}\left\|\psi_{j}\right\|_{\mathrm{L}^{p}\left(t_{0}, T\right)}\left\|\eta_{j}\right\|_{\mathrm{L}^{p}(\Omega)}<\infty \text { and } \\
& \left\|\mathrm{e}^{-\frac{1}{3} K_{a}\left(t, \boldsymbol{\xi}_{N}\right)}\right\|_{\mathrm{L}^{q}(\Omega)} \leq C, \text { for all } N \geq 1 \text { and } t \in\left[t_{0}, T\right] .
\end{aligned}
$$


Then the sequence $\left\{f_{x_{N}(t)}(x)\right\}_{N=1}^{\infty}$ given by (4.12) converges in $\mathrm{L}^{\infty}\left(J \times\left[t_{0}, T\right]\right)$ for every bounded set $J \subseteq \mathbb{R} \backslash[-\delta, \delta]$, for every $\delta>0$, to the density $f_{x(t)}(x)$ of the solution process $x(t, \omega)$ given by (4.2).

Proof. By (4.10), $f_{y_{0}}$ is Lipschitz on $\mathbb{R}$. As a consequence of [20, Th. 2.4], the limit 4.13) holds in $\mathrm{L}^{\infty}\left(J \times\left[t_{0}, T\right]\right)$. Then,

$$
\begin{gathered}
\left\|f_{x_{N}(t)}(x)-f_{x(t)}(x)\right\|_{\mathrm{L}^{\infty}\left(J \times\left[t_{0}, T\right]\right)}=\left\|\left(f_{y_{N}(t)}\left(x^{\frac{1}{3}}\right)-f_{y(t)}\left(x^{\frac{1}{3}}\right)\right) \frac{1}{3 x^{\frac{2}{3}}}\right\|_{L^{\infty}\left(J \times\left[t_{0}, T\right]\right)} \\
\leq \frac{1}{3 \delta^{\frac{2}{3}}}\left\|f_{y_{N}(t)}\left(x^{\frac{1}{3}}\right)-f_{y(t)}\left(x^{\frac{1}{3}}\right)\right\|_{L^{\infty}\left(J \times\left[t_{0}, T\right]\right)} \stackrel{N \rightarrow \infty}{\longrightarrow} 0 .
\end{gathered}
$$

This concludes the proof of the theorem.

Theorem 4.11 Assume that

$H 1: a, b \in \mathrm{L}^{2}\left(\left[t_{0}, T\right] \times \Omega\right), x_{0} \in \mathrm{L}^{\frac{2}{3}}(\Omega)$;

H2: $x_{0}, \eta_{1},\left(\xi_{1}, \ldots, \xi_{N}, \eta_{2}, \ldots, \eta_{N}\right)$ are absolutely continuous and independent, $N \geq 1$

H3: the density function of $\eta_{1}, f_{\eta_{1}}$, is Lipschitz on $\mathbb{R}$;

$H_{4}: \xi_{1}, \xi_{2}, \ldots$ have compact support in $[-A, A](A>0), \psi_{1}>0$ on $\left(t_{0}, T\right)$.

Then, for each fixed $t \in\left(t_{0}, T\right]$, the sequence $\left\{f_{x_{N}(t)}(x)\right\}_{N=1}^{\infty}$ given by (4.12) converges in $\mathrm{L}^{\infty}(J)$ for every bounded set $J \subseteq \mathbb{R} \backslash[-\delta, \delta]$, for every $\delta>0$, to the density $f_{x(t)}(x)$ of the solution process $x(t, \omega)$ given by (4.2).

Proof. Since $y_{0}(\omega)=x_{0}(\omega)^{1 / 3}, \mathbb{E}\left[y_{0}^{2}\right]=\mathbb{E}\left[x_{0}^{2 / 3}\right]<\infty$ by hypothesis H1. Then, $y_{0} \in \mathrm{L}^{2}(\Omega)$ and therefore hypothesis H1 of [20, Th. 2.7] holds. In fact, the four hypotheses of [20, Th. 2.7] are fulfilled. Hence, fixed $t \in\left(t_{0}, T\right]$, the limit in 4.13 holds in $\mathrm{L}^{\infty}(J)$, then 4.16 follows and we are done.

\subsubsection{Comments on the hypotheses of the theorems}

We comment on some examples where the hypotheses of the theorems previously established hold. We point out that these comments will be very useful later in the examples exhibited in Section 4.4 .

(c1) In [20, pp. 29-30], it was proved that, if $a(t, \omega)$ is Gaussian or if $\xi_{1}, \xi_{2}, \ldots$ have a common compact support, then given any $c \in \mathbb{R}$, there is a constant $C>0$ such that the inequality $\mathbb{E}\left[\mathrm{e}^{c K_{a}\left(t, \boldsymbol{\xi}_{N}\right)}\right] \leq C$ holds for all $N \geq 1$ and $t \in\left[t_{0}, T\right]$. 
(c2) The continuity on $\mathbb{R}$ is satisfied, for instance, by the density function of the distributions $\operatorname{Normal}\left(\mu, \sigma^{2}\right), \mu \in \mathbb{R}$ and $\sigma^{2}>0 ; \operatorname{Beta}(\alpha, \beta), \alpha>1$ and $\beta>1 ; \operatorname{Gamma}(\alpha, \beta), \alpha>1$ and $\beta>0$. The a.e. continuity from Theorem 4.9 is satisfied in more cases: $\operatorname{Beta}(\alpha, \beta), \alpha \geq 1$ and $\beta \geq 1$; $\operatorname{Uniform}(\alpha, \beta), \alpha<\beta$; $\operatorname{Gamma}(\alpha, \beta), \alpha \geq 1$ and $\beta>0$ (in particular, Exponential $(\beta))$; truncated normal distribution; etc.

(c3) In hypothesis $\mathrm{H} 3$ of Theorem 4.6 , the hypotheses $f_{x_{0}}$ continuous on $\mathbb{R}$ and $f_{x_{0}}(x) \leq C /|x|^{2 / 3}$ are independent, merely because there are unbounded continuous density functions.

(c4) Hypothesis $\mathrm{H} 4$ of Theorem 4.10 is satisfied, for example, when $b(t, \omega)$ is a standard Brownian motion or a Brownian bridge, as it was seen in [20. pp. 30-31] with $p=3$.

\subsubsection{Approximation of the expectation and variance of the solution stochastic process}

We have seen that, under some assumptions, 4.15 holds. We would like to derive conditions under which the expectation and variance of $x(t, \omega)$ can be approximated:

$$
\mathbb{E}\left[x_{N}(t, \omega)\right]=\int_{\mathbb{R}} x f_{x_{N}(t)}(x) \mathrm{d} x \stackrel{N \rightarrow \infty}{\longrightarrow} \int_{\mathbb{R}} x f_{x(t)}(x) \mathrm{d} x=\mathbb{E}[x(t, \omega)]
$$

and

$$
\begin{aligned}
\mathbb{V}\left[x_{N}(t, \omega)\right] & =\int_{\mathbb{R}} x^{2} f_{x_{N}(t)}(x) \mathrm{d} x-\mathbb{E}\left[x_{N}(t, \omega)\right]^{2} \stackrel{N \rightarrow \infty}{\longrightarrow} \int_{\mathbb{R}} x^{2} f_{x(t)}(x) \mathrm{d} x-\mathbb{E}[x(t, \omega)]^{2} \\
& =\mathbb{V}[x(t, \omega)] .
\end{aligned}
$$

From [20, p. 13], $y_{N}(t, \omega) \rightarrow y(t, \omega)$ a.s., therefore $x_{N}(t, \omega) \rightarrow x(t, \omega)$ a.s. This implies that $x_{N}(t, \omega) \rightarrow x(t, \omega)$ in probability. By [8, p. 338 Corollary] or [137, Th. 2.4], if we check that

$$
\sup _{N \geq 1} \mathbb{E}\left[\left|x_{N}(t, \omega)\right|^{2+\epsilon}\right]<\infty
$$

for some $\epsilon>0$, then 4.17, 4.18 and $x(t, \omega) \in \mathrm{L}^{2}(\Omega)$ will follow.

In what follows, we will use a consequence of Jensen's inequality [8, p. 80]: if $a_{1}, \ldots, a_{m} \geq 0$ and $p \geq 1$, then $\left(a_{1}+\ldots+a_{m}\right)^{p} \leq m^{p-1}\left(a_{1}^{p}+\ldots+a_{m}^{p}\right)$. For ease of notation, we will denote by $C_{\epsilon}$ any positive constant only depending on $\epsilon$. 
By the triangular, Jensen's and Hölder's inequalities,

$$
\begin{aligned}
& \mathbb{E}\left[\left|x_{N}(t)\right|^{2+\epsilon}\right]=\mathbb{E}\left[\left|x_{0}^{\frac{1}{3}} \mathrm{e}^{\frac{1}{3} K_{a}\left(t, \boldsymbol{\xi}_{N}\right)}+\frac{1}{3} \int_{t_{0}}^{t} S_{b}\left(s, \boldsymbol{\eta}_{N}\right) \mathrm{e}^{\frac{1}{3}\left(K_{a}\left(t, \boldsymbol{\xi}_{N}\right)-K_{a}\left(s, \boldsymbol{\xi}_{N}\right)\right)} \mathrm{d} s\right|^{3(2+\epsilon)}\right] \\
& \leq C_{\epsilon}\left(\mathbb{E}\left[\left|x_{0}^{\frac{1}{3}} \mathrm{e}^{\frac{1}{3} K_{a}\left(t, \boldsymbol{\xi}_{N}\right)}\right|^{3(2+\epsilon)}\right]+\mathbb{E}\left[\left|\frac{1}{3} \int_{t_{0}}^{t} S_{b}\left(s, \boldsymbol{\eta}_{N}\right) \mathrm{e}^{\frac{1}{3}\left(K_{a}\left(t, \boldsymbol{\xi}_{N}\right)-K_{a}\left(s, \boldsymbol{\xi}_{N}\right)\right)} \mathrm{d} s\right|^{3(2+\epsilon)}\right]\right) \\
& \leq C_{\epsilon}\left(\mathbb{E}\left[\left|x_{0}\right|^{2+\epsilon} \mathrm{e}^{(2+\epsilon) K_{a}\left(t, \boldsymbol{\xi}_{N}\right)}\right]+\mathbb{E}\left[\int_{t_{0}}^{t}\left|S_{b}\left(s, \boldsymbol{\eta}_{N}\right)\right|^{3(2+\epsilon)} \mathrm{e}^{(2+\epsilon)\left(K_{a}\left(t, \boldsymbol{\xi}_{N}\right)-K_{a}\left(s, \boldsymbol{\xi}_{N}\right)\right)} \mathrm{d} s\right]\right) .
\end{aligned}
$$

If $\xi_{1}, \xi_{2}, \ldots$ have compact support in a common interval, then there is a constant $C_{\epsilon}>0$ such that $\mathrm{e}^{(2+\epsilon) K_{a}\left(t, \boldsymbol{\xi}_{N}\right)} \leq C_{\epsilon}$, for all $N \geq 1$ and $t \in\left[t_{0}, T\right]$. This is a consequence of [20, p. 30]. In this case, from 4.20,

$$
\mathbb{E}\left[\left|x_{N}(t)\right|^{2+\epsilon}\right] \leq C_{\epsilon}\left(\mathbb{E}\left[\left|x_{0}\right|^{2+\epsilon}\right]+\mathbb{E}\left[\int_{t_{0}}^{t}\left|S_{b}\left(s, \boldsymbol{\eta}_{N}\right)\right|^{3(2+\epsilon)} \mathrm{d} s\right]\right) .
$$

Thus, it suffices to have $\left\|x_{0}\right\|_{\mathrm{L}^{2+\epsilon}(\Omega)}<\infty$ and

$$
\left\|\mu_{b}\right\|_{L^{3(2+\epsilon)}\left(\left[t_{0}, T\right]\right)}+\sum_{i=1}^{\infty} \sqrt{\gamma_{i}}\left\|\psi_{i}\right\|_{L^{3(2+\epsilon)}\left(\left[t_{0}, T\right]\right)}\left\|\eta_{i}\right\|_{L^{3(2+\epsilon)}(\Omega)}<\infty
$$

to ensure that 4.19 holds.

Otherwise, if $\xi_{1}, \xi_{2}, \ldots$ do not have compact support in a common interval, one continues from 4.20 by applying Hölder's inequality with exponents $r_{1}=$ $1+\delta>1$ and $r_{2}=(1+\delta) / \delta$ :

$$
\begin{aligned}
& \mathbb{E}\left[\left|x_{N}(t)\right|^{2+\epsilon}\right] \leq C_{\epsilon}\left(\mathbb{E}\left[\left|x_{0}\right|^{(2+\epsilon)(1+\delta)}\right]^{\frac{1}{1+\delta}} \mathbb{E}\left[\mathrm{e}^{(2+\epsilon) r_{2} K_{a}\left(t, \boldsymbol{\xi}_{N}\right)}\right]^{\frac{1}{r_{2}}}\right. \\
& \left.+\int_{t_{0}}^{t} \mathbb{E}\left[\left|S_{b}\left(s, \boldsymbol{\eta}_{N}\right)\right|^{3(2+\epsilon)(1+\delta)}\right]^{\frac{1}{1+\delta}} \mathbb{E}\left[\mathrm{e}^{(2+\epsilon) r_{2}\left(K_{a}\left(t, \boldsymbol{\xi}_{N}\right)-K_{a}\left(s, \boldsymbol{\xi}_{N}\right)\right)}\right]^{\frac{1}{r_{2}}} \mathrm{~d} s\right) .
\end{aligned}
$$

If $a(t, \omega)$ is a Gaussian process, then, by the first paragraph of Subsection 4.3.2 and Hölder's inequality,

$$
\begin{aligned}
& \mathbb{E}\left[\left|x_{N}(t)\right|^{2+\epsilon}\right] \leq C_{\epsilon, \delta}\left(\mathbb{E}\left[\left|x_{0}\right|^{(2+\epsilon)(1+\delta)}\right]^{\frac{1}{1+\delta}}+\int_{t_{0}}^{t} \mathbb{E}\left[\left|S_{b}\left(s, \boldsymbol{\eta}_{N}\right)\right|^{3(2+\epsilon)(1+\delta)}\right]^{\frac{1}{1+\delta}} \mathrm{d} s\right) \\
& \leq C_{\epsilon, \delta}\left(\mathbb{E}\left[\left|x_{0}\right|^{(2+\epsilon)(1+\delta)}\right]^{\frac{1}{1+\delta}}+\left(\int_{t_{0}}^{t} \mathbb{E}\left[\left|S_{b}\left(s, \boldsymbol{\eta}_{N}\right)\right|^{3(2+\epsilon)(1+\delta)}\right] \mathrm{d} s\right)^{\frac{1}{1+\delta}}\right) .
\end{aligned}
$$


Thereby, if $\left\|x_{0}\right\|_{\mathrm{L}^{2+s}(\Omega)}<\infty$ and

$$
\left\|\mu_{b}\right\|_{\mathrm{L}^{6+s}\left(\left[t_{0}, T\right]\right)}+\sum_{i=1}^{\infty} \sqrt{\gamma_{i}}\left\|\psi_{i}\right\|_{\mathrm{L}^{6+s}\left(\left[t_{0}, T\right]\right)}\left\|\eta_{i}\right\|_{\mathrm{L}^{6+s}(\Omega)}<\infty,
$$

4.19 holds.

Summarizing, the following theorem has been established:

Theorem 4.12 If $a(t, \omega)$ is a Gaussian process or $\xi_{1}, \xi_{2}, \ldots$ have a common compact support, if $\left\|x_{0}\right\|_{\mathrm{L}^{2+s}(\Omega)}<\infty$ and if

$$
\left\|\mu_{b}\right\|_{L^{6+s}\left(\left[t_{0}, T\right]\right)}+\sum_{i=1}^{\infty} \sqrt{\gamma_{i}}\left\|\psi_{i}\right\|_{L^{6+s}\left(\left[t_{0}, T\right]\right)}\left\|\eta_{i}\right\|_{L^{6+s}(\Omega)}<\infty
$$

for some $s>0$, then $x(t, \omega) \in \mathrm{L}^{2}(\Omega)$ and $x_{N}(t, \omega)$ tends in $\mathrm{L}^{2}(\Omega)$ to $x(t, \omega)$, for each $t \in\left[t_{0}, T\right]$. As a consequence, $\mathbb{E}\left[x_{N}(t, \omega)\right] \rightarrow \mathbb{E}[x(t, \omega)]$ and $\mathbb{V}\left[x_{N}(t, \omega)\right] \rightarrow$ $\mathbb{V}[x(t, \omega)]$ as $N \rightarrow \infty$, for each $t \in\left[t_{0}, T\right]$.

\subsection{Numerical examples}

In this section we show examples where the theoretical findings of this chapter are illustrated. We choose specific stochastic processes $a(t, \omega)$ and $b(t, \omega)$ (via their Karhunen-Loève expansions) and an initial condition $x_{0}(\omega)$ in the random Bertalanffy model (4.1), and then we compute the approximating density function $f_{x_{N}(t)}(x)$ given by 4.12 for different values of $N \geq 1$. We also check that it converges to a function, which will be $f_{x(t)}(x)$, as an application of Theorems 4.6, 4.7, 4.9, 4.10 or 4.11. We alert the reader that comments (c1)(c4) listed in Subsection 4.3.2 will be extensively used throughout this section to check that the hypotheses of the involved theorems hold within the context of each example.

To compute $f_{x_{N}(t)}(x)$, we have used the software Mathematica ${ }^{\circledR}$. We have programed a Monte Carlo procedure to compute the expectation in (4.12): fixed $N \geq 1$, we obtain $M$ realizations of each random variable $\xi_{1}, \ldots, \xi_{N}, \eta_{1}, \ldots, \eta_{N}$ :

$$
\begin{gathered}
\text { realizations of } \xi_{1}: \xi_{1}^{(1)}, \ldots, \xi_{1}^{(M)}, \\
\ldots \\
\text { realizations of } \eta_{N}: \eta_{N}^{(1)}, \ldots, \eta_{N}^{(M)} .
\end{gathered}
$$


We denote $\boldsymbol{\xi}_{N}^{(i)}=\left(\xi_{1}^{(i)}, \ldots, \xi_{N}^{(i)}\right)$ and $\boldsymbol{\eta}_{N}^{(i)}=\left(\eta_{1}^{(i)}, \ldots, \eta_{N}^{(i)}\right), i=1, \ldots, M$. Then

$$
\begin{aligned}
f_{x_{N}(t)}(x) & \approx \frac{1}{x^{\frac{2}{3}}} \cdot \frac{1}{M} \sum_{i=1}^{M}\left[f_{x_{0}}\left(\left\{x^{\frac{1}{3}} \mathrm{e}^{-\frac{1}{3} K_{a}\left(t, \boldsymbol{\xi}_{N}^{(i)}\right)}-\frac{1}{3} \int_{t_{0}}^{t} S_{b}\left(s, \boldsymbol{\eta}_{N}^{(i)}\right) \mathrm{e}^{-\frac{1}{3} K_{a}\left(s, \boldsymbol{\xi}_{N}^{(i)}\right)} \mathrm{d} s\right\}^{3}\right)\right. \\
& \left.\times\left\{x^{\frac{1}{3}} \mathrm{e}^{-\frac{1}{3} K_{a}\left(t, \boldsymbol{\xi}_{N}^{(i)}\right)}-\frac{1}{3} \int_{t_{0}}^{t} S_{b}\left(s, \boldsymbol{\eta}_{N}^{(i)}\right) \mathrm{e}^{-\frac{1}{3} K_{a}\left(s, \boldsymbol{\xi}_{N}^{(i)}\right)} \mathrm{d} s\right\}^{2} \mathrm{e}^{-\frac{1}{3} K_{a}\left(t, \boldsymbol{\xi}_{N}^{(i)}\right)}\right],
\end{aligned}
$$

with convergence as $M \rightarrow \infty$, by the Law of Large Numbers. We take $M$ large enough in such a way that expression (4.22) coincides for orders of truncation $M$ and $M^{\prime}>M$ (convergence in 4.22$)$ ).

We perform three examples in what follows. In the first example, both processes $a(t, \omega)$ and $b(t, \omega)$ are Gaussian. In the second and third examples, the processes involved may not be Gaussian.

Example 4.13 Let

$$
a(t, \omega)=\sum_{j=1}^{\infty} \frac{\sqrt{2}}{\left(j-\frac{1}{2}\right) \pi} \sin \left(t\left(j-\frac{1}{2}\right) \pi\right) \xi_{j}(\omega),
$$

where $\xi_{1}, \xi_{2}, \ldots$ are independent and $\operatorname{Normal}(0,1)$ random variables, be a standard Brownian motion on $\left[t_{0}, T\right]=[0,1][89$, Exercise 5.12]. Let

$$
b(t, \omega)=\sum_{i=1}^{\infty} \frac{\sqrt{2}}{i \pi} \sin (t i \pi) \eta_{i}(\omega),
$$

where $\xi_{1}, \xi_{2}, \ldots, \eta_{1}, \eta_{2}, \ldots$ are independent and $\operatorname{Normal}(0,1)$ distributed random variables, be a standard Brownian bridge on $[0,1]$ [89, Example 5.30]. The sums defining $a(t, \omega)$ and $b(t, \omega)$ converge in $\mathrm{L}^{2}([0,1] \times \Omega)$. Let $x_{0} \sim \operatorname{Beta}(4,6)$. It is assumed $x_{0}, \xi_{1}, \xi_{2}, \ldots$ and $\eta_{1}, \eta_{2}, \ldots$ to be independent.

Since $a(\cdot, \omega)$ and $b(\cdot, \omega)$ are continuous on $[0,1]$, by Theorem 4.1 , the stochastic process $x(t, \omega)$ given by $(4.2)$ has $C^{1}\left(\left[t_{0}, T\right]\right)$ sample paths that satisfy the random Bertalanffy model (4.1). Moreover, by Theorem 4.2 and the subsequent Example 4.3, $x(t, \omega)$ is a solution to the random Bertalanffy model (4.1) in the mean square sense.

The hypotheses of Theorem 4.6 and Theorem 4.10 are satisfied. Indeed, $f_{x_{0}}$ is continuous on $\mathbb{R}$ by $(\mathrm{c} 2)$ and $f_{x_{0}}(x) \leq C /|x|^{2 / 3}$ holds because $f_{x_{0}}$ is continuous with compact support, therefore $\mathrm{H} 3$ of Theorem 4.6 fulfills. Hypothesis $\mathrm{H} 4$ of Theorem 4.6 is satisfied because $a(t, \omega)$ is Gaussian and (c1). Regarding Theorem 4.10, hypothesis H3 holds, since $g(x):=f_{x_{0}}\left(x^{3}\right) x^{2}=504 x^{11}(1-$ 
$\left.x^{3}\right)^{5} \mathbb{1}_{(0,1)}(x)$ is differentiable on $\mathbb{R}$, with $g^{\prime}(x)=504 x^{10}\left(1-x^{3}\right)^{4}\left(11\left(1-x^{3}\right)-\right.$ $\left.15 x^{3}\right) \mathbb{1}_{(0,1)}(x)$ bounded on $\mathbb{R}$. Moreover, $\mathrm{H} 4$ is fulfilled by $(\mathrm{c} 1)$ applied to $a(t, \omega)$ and (c4) applied to $b(t, \omega)$.

Hence, the sequence $\left\{f_{x_{N}(t)}(x)\right\}$ converges in $\mathrm{L}^{\infty}\left(J \times\left[t_{0}, T\right]\right)$ for every bounded set $J \subseteq \mathbb{R} \backslash[-\delta, \delta]$, for every $\delta>0$, to the density $f_{x(t)}(x)$ of the solution process $x(t, \omega)$.

The assumptions of Theorem 4.12 are fulfilled. Indeed, by taking $s=1$ in 4.21,

$$
\begin{aligned}
\left\|\mu_{b}\right\|_{\mathrm{L}^{7}([0,1])}+\sum_{i=1}^{\infty} \sqrt{\gamma_{i}}\left\|\psi_{i}\right\|_{\mathrm{L}^{7}([0,1])}\left\|\eta_{i}\right\|_{\mathrm{L}^{7}(\Omega)} & =M_{7} \sum_{i=1}^{\infty} \frac{\sqrt{2}}{i \pi}\left(\int_{0}^{1}|\sin (t i \pi)|^{7} \mathrm{~d} t\right)^{\frac{1}{7}} \\
& =M_{7} \sum_{i=1}^{\infty} \frac{1}{i \pi} \frac{2 \sqrt[14]{2} \sqrt[7]{\frac{1}{i}}}{\sqrt[7]{35 \pi}}<\infty, \quad \text { (4.23) }
\end{aligned}
$$

where $M_{7}=\|Z\|_{\mathrm{L}^{\top}(\Omega)}$, being $Z \sim \operatorname{Normal}(0,1)$. Hence, $\mathbb{E}\left[x_{N}(t, \omega)\right] \rightarrow \mathbb{E}[x(t, \omega)]$ and $\mathbb{V}\left[x_{N}(t, \omega)\right] \rightarrow \mathbb{V}[x(t, \omega)]$ as $N \rightarrow \infty$.

In Figure 4.1, we observe the graph of $f_{x_{N}(0.5)}(x), x \in \mathbb{R}$, for $N=1,2,3,4,5,6$. The theoretical convergence of the sequence $\left\{f_{x_{N}(0.5)}(x)\right\}_{N=1}^{\infty}$ agrees with the numerical results of Figure 4.1. In Table 4.1, we have presented the expectation and variance for $N=1,2,3,4,5,6$, by using the formulas $\mathbb{E}\left[x_{N}(0.5, \omega)\right]=$ $\int_{\mathbb{R}} x f_{x_{N}(0.5)}(x) \mathrm{d} x$ and $\mathbb{V}\left[x_{N}(0.5, \omega)\right]=\int_{\mathbb{R}} x^{2} f_{x_{N}(0.5)}(x) \mathrm{d} x-\mathbb{E}\left[x_{N}(0.5, \omega)\right]^{2}$.

\begin{tabular}{|c|c|c|c|c|c|c|}
\hline$N$ & 1 & 2 & 3 & 4 & 5 & 6 \\
\hline $\mathbb{E}\left[x_{N}(0.5, \omega)\right]$ & 0.4109 & 0.4139 & 0.4145 & 0.4145 & 0.4146 & 0.4146 \\
\hline $\mathbb{V}\left[x_{N}(0.5, \omega)\right]$ & 0.0345 & 0.0391 & 0.0396 & 0.0396 & 0.0397 & 0.0397 \\
\hline
\end{tabular}

Table 4.1: $\mathbb{E}\left[x_{N}(0.5, \omega)\right]$ and $\mathbb{V}\left[x_{N}(0.5, \omega)\right]$ for $N=1,2,3,4,5,6$. Example 4.13

Example 4.14 We work on $\left[t_{0}, T\right]=[0,1]$. Let

$$
a(t, \omega)=\sum_{j=1}^{\infty} \frac{\sqrt{2}}{j^{3}} \sin (t j \pi) \xi_{j}(\omega),
$$

where $\xi_{1}, \xi_{2}, \ldots$ are independent with distribution $\operatorname{Uniform}(-\sqrt{3}, \sqrt{3})$. The sum in 4.24 converges in $\mathrm{L}^{2}([0,1] \times \Omega)$ : given $M<N$, by using Pythagoras's 


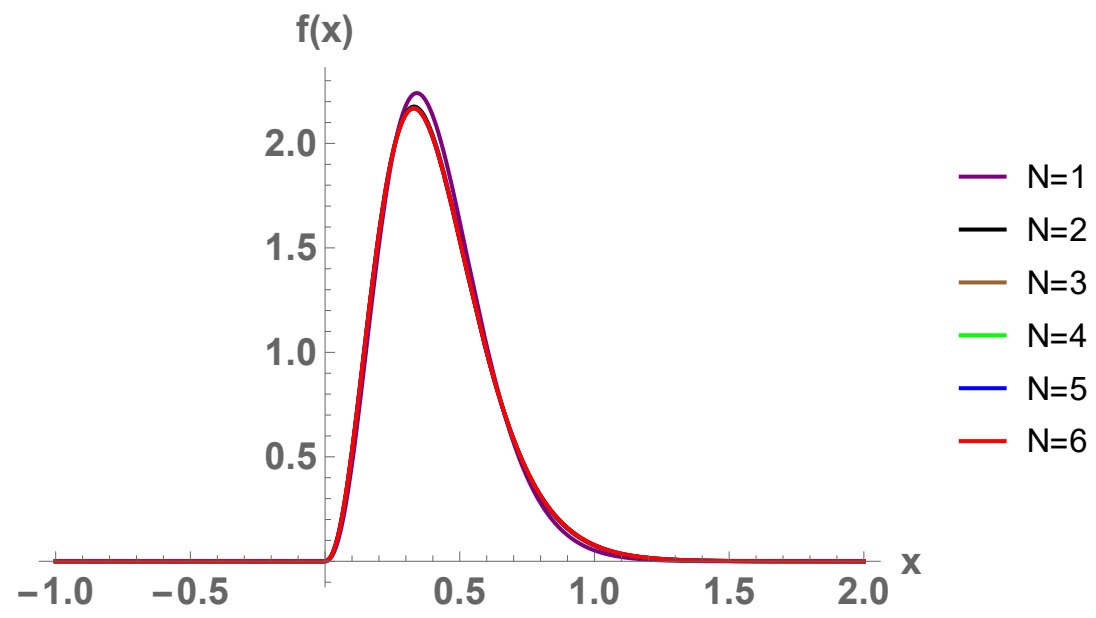

Figure 4.1: Density $f_{x_{N}(0.5)}(x)$ for $N=1,2,3,4,5,6$. Example 4.13

Theorem, we have

$$
\left\|\sum_{j=M+1}^{N} \frac{\sqrt{2}}{j^{3}} \sin (t j \pi) \xi_{j}(\omega)\right\|_{L^{2}([0,1] \times \Omega)}^{2}=\sum_{j=M+1}^{N} \frac{2}{j^{6}},
$$

and since $\sum_{j=1}^{\infty} 2 / j^{6}<\infty$, the sequence $\left\{\sum_{j=1}^{N}\left(\sqrt{2} / j^{3}\right) \sin (t j \pi) \xi_{j}(\omega)\right\}_{N=1}^{\infty}$ of partial sums is Cauchy in $\mathrm{L}^{2}([0,1] \times \Omega)$, therefore convergent.

Let

$$
b(t, \omega)=\sum_{i=1}^{\infty} \frac{\sqrt{2}}{i^{4}+6} \sin (t i \pi) \eta_{i}(\omega),
$$

where $\eta_{1}, \eta_{2}, \ldots \sim \operatorname{Normal}(0,1)$ are independent. The sum defining $b(t, \omega)$ in 4.25) exists in $\mathrm{L}^{2}([0,1] \times \Omega)$, reasoning as in 4.24.

Let $x_{0}$ follow an Exponential(2) distribution. It is assumed $x_{0}, \xi_{1}, \xi_{2}, \ldots$ and $\eta_{1}, \eta_{2}, \ldots$ to be independent.

By Theorem 4.1, the process $x(t, \omega)$ given by 4.2 has absolutely continuous sample paths that solve the random Bertalanffy model (4.1). In fact, $a(t, \omega)$ and $b(t, \omega)$ have continuous sample paths. To prove the continuity for $a(t, \omega)$, we bound

$$
\frac{\sqrt{2}}{j^{3}}\left|\sin (t j \pi) \xi_{j}(\omega)\right| \leq \frac{\sqrt{6}}{j^{3}}
$$


with $\sum_{j=1}^{\infty} 1 / j^{3}<\infty$, and by using Weierstrass M-test for uniform convergence of series [113, Th. 7.10], we deduce that the series in (4.24) converges uniformly on $[0,1]$, so $a(t, \omega)$ has continuous sample paths. To prove the continuity for $b(t, \omega)$, one has to work a bit more, since $\eta_{i}$ is not bounded. Notice that

$$
\mathbb{P}\left(\left|\eta_{i}\right| \geq i\right)=2 \int_{i}^{\infty} \frac{1}{\sqrt{2 \pi}} \mathrm{e}^{-\frac{x^{2}}{2}} \mathrm{~d} x \leq \frac{2}{\sqrt{2 \pi}} \int_{i}^{\infty} \frac{x}{i} \mathrm{e}^{-\frac{x^{2}}{2}} \mathrm{~d} x=\frac{2}{\sqrt{2 \pi} i} \mathrm{e}^{-\frac{i^{2}}{2}} \leq \mathrm{e}^{-\frac{i^{2}}{2}},
$$

therefore $\sum_{i=1}^{\infty} \mathbb{P}\left(\left|\eta_{i}\right| \geq i\right) \leq \sum_{i=1}^{\infty} \mathrm{e}^{-\frac{i^{2}}{2}}<\infty$. By Borel-Cantelli lemma [ Th. 4.3], for a.e. $\omega \in \Omega$, there exists an $i_{0}(\omega) \geq 1$ such that, for all $i \geq i_{0}(\omega)$, $\left|\eta_{i}(\omega)\right| \leq i$. Thus, for all $i \geq i_{0}(\omega)$,

$$
\frac{\sqrt{2}}{i^{4}+6}\left|\sin (t i \pi) \eta_{i}(\omega)\right| \leq \frac{\sqrt{2} i}{i^{4}+6} .
$$

Since $\sum_{i=1}^{\infty} i /\left(i^{4}+6\right)<\infty$, by Weierstrass M-test for uniform convergence of series, we derive that the series defining $b(t, \omega)$ in (4.25) converges uniformly on $[0,1]$, therefore $b(t, \omega)$ has continuous sample paths. As a consequence, by Theorem 4.1, $x(t, \omega)$ has $C^{1}([0,1])$ sample paths that solve the random Bertalanffy model 4.1.

The process $x(t, \omega)$ is a mean square solution to the random Bertalanffy model 4.1), as the hypotheses of Theorem 4.2 are accomplished. Indeed, $a(t, \omega)$ is continuous in the $\mathrm{L}^{12}(\Omega)$ sense, since

$$
\|a(t+h, \omega)-a(t, \omega)\|_{\mathrm{L}^{12}(\Omega)} \leq \sqrt{3} \sum_{j=1}^{\infty} \frac{\sqrt{2}}{j^{3}}|\sin ((t+h) j \pi)-\sin (t j \pi)| \stackrel{h \rightarrow 0}{\longrightarrow} 0,
$$

where we have used the fact that $\left|\xi_{j}(\omega)\right| \leq \sqrt{3}$ and, for the last limit, the Dominated Convergence Theorem [113, result 11.32, p. 321] applied to the last series. On the other hand,

$$
\left|\int_{0}^{t} a(s, \omega) \mathrm{d} s\right| \leq \int_{0}^{t}|a(s, \omega)| \mathrm{d} s \leq \sum_{j=1}^{\infty} \frac{\sqrt{2}}{j^{3}} \sqrt{3}<\infty,
$$

so the third hypothesis of Theorem 4.2 holds. The other process, $b(t, \omega)$, is $\mathrm{L}^{12}(\Omega)$-continuous:

$$
\|b(t+h, \omega)-b(t, \omega)\|_{\mathrm{L}^{12}(\Omega)} \leq M_{12} \sum_{i=1}^{\infty} \frac{\sqrt{2}}{i^{4}+6}|\sin ((t+h) j \pi)-\sin (t j \pi)| \stackrel{h \rightarrow 0}{\longrightarrow} 0,
$$

where $M_{12}=\|Z\|_{\mathrm{L}^{12}(\Omega)}$, being $Z \sim \operatorname{Normal}(0,1)$. For the limit, the Dominated Convergence Theorem has been applied again. Thus, the hypotheses of Theorem 4.2 hold, as stated, and the process $x(t, \omega)$ is a mean square solution. 
Theorem 4.7 and Theorem 4.11 are applicable. Indeed, hypothesis H3 of Theorem 4.7 is satisfied, because $f_{\eta_{1}}$ is the density function of a normal distribution, which is continuous and bounded. Assumption H4 of Theorem 4.7 is accomplished, because the density function of a $\operatorname{Uniform}(-\sqrt{3}, \sqrt{3})$ distribution has compact support and $\psi_{1}(t)=\sqrt{2} \sin (t \pi)>0$ on $(0,1)$. Concerning Theorem 4.11, H3 holds because the density function of a normal distribution is Lipschitz and H4 is the same as in Theorem 4.7.

Therefore, for each $t \in\left(t_{0}, T\right]$, the sequence $\left\{f_{x_{N}(t)}(x)\right\}$ converges in $\mathrm{L}^{\infty}(J)$ for every bounded set $J \subseteq \mathbb{R} \backslash[-\delta, \delta]$, for every $\delta>0$, to the density $f_{x(t)}(x)$ of the solution process $x(t, \omega)$.

The assumptions of Theorem 4.12 are fulfilled, by doing similar computations to the ones in 4.23 . As a consequence, $\mathbb{E}\left[x_{N}(t, \omega)\right] \rightarrow \mathbb{E}[x(t, \omega)]$ and $\mathbb{V}\left[x_{N}(t, \omega)\right] \rightarrow \mathbb{V}[x(t, \omega)]$ as $N \rightarrow \infty$.

In Figure 4.2 we show the graph of $f_{x_{N}(0.3)}(x)$ for $N=1,2,3,4,5,6$. In Table 4.2 , we approximate the mean and variance of $x(t, \omega)$, with orders of truncation $N=1,2,3,4,5,6$. The numerical results agree with our theoretical findings.

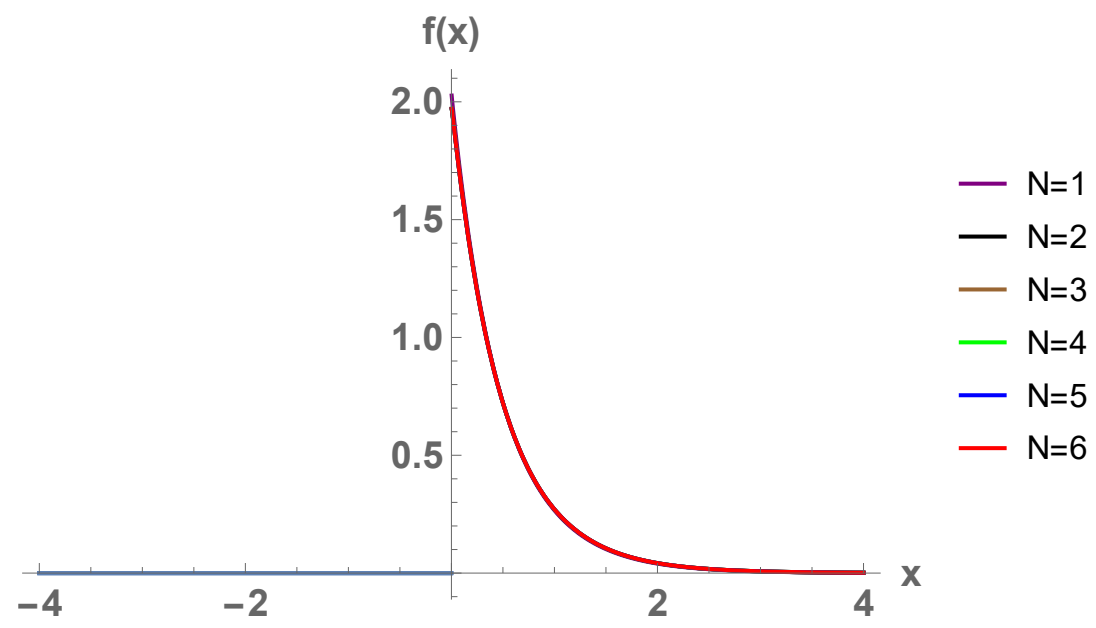

Figure 4.2: Density $f_{x_{N}(0.3)}(x)$ for $N=1,2,3,4,5,6$. Example 4.14 


\begin{tabular}{|c|c|c|c|c|c|c|}
\hline$N$ & 1 & 2 & 3 & 4 & 5 & 6 \\
\hline $\mathbb{E}\left[x_{N}(0.3, \omega)\right]$ & 0.5071 & 0.5078 & 0.5076 & 0.5076 & 0.5076 & 0.5076 \\
\hline $\mathbb{V}\left[x_{N}(0.3, \omega)\right]$ & 0.2754 & 0.2769 & 0.2768 & 0.2768 & 0.2768 & 0.2768 \\
\hline
\end{tabular}

Table 4.2: $\mathbb{E}\left[x_{N}(0.3, \omega)\right]$ and $\mathbb{V}\left[x_{N}(0.3, \omega)\right]$ for $N=1,2,3,4,5,6$. Example 4.14

Example 4.15 We work on $\left[t_{0}, T\right]=[0,1]$. Let

$$
a(t, \omega)=\sum_{j=1}^{\infty} \frac{\sqrt{2}}{j} \sin (t j \pi) \xi_{j}(\omega),
$$

where $\xi_{1}, \xi_{2}, \ldots$ are independent with distribution $\operatorname{Uniform}(-\sqrt{3}, \sqrt{3})$. Let

$$
b(t, \omega)=\sum_{i=1}^{\infty} \frac{\sqrt{2}}{i^{\frac{3}{2}}} \sin (t i \pi) \eta_{i}(\omega),
$$

where $\eta_{1}, \eta_{2}, \ldots$ are independent having distribution $\operatorname{Uniform}(-\sqrt{3}, \sqrt{3})$. Let the initial condition be $x_{0} \sim \operatorname{Uniform}(-\sqrt{3}, \sqrt{3})$. It is assumed $x_{0}, \xi_{1}, \xi_{2}, \ldots$ and $\eta_{1}, \eta_{2}, \ldots$ to be independent. As in Example 4.14 , both series converge in $\mathrm{L}^{2}([0,1] \times \Omega)$, as a consequence of Pythagoras's Theorem.

The process $x(t, \omega)$ has absolutely continuous sample paths that solve the random Bertalanffy model, by Theorem 4.1 .

Let us see that the hypotheses of Theorem 4.9 are satisfied. The density function $f_{x_{0}}$ is a.e. continuous (see (c2)). The inequality $f_{x_{0}}(x) \leq C /|x|^{2 / 3}$ holds because $x_{0}$ has compact support, so H3 is satisfied. Hypotheses $\mathrm{H} 4$ is a consequence of $(\mathrm{c} 1)$. Finally, H5 holds because $\psi_{1}(s)=\sin (s \pi)>0$ for $s \in(0,1)$. Thus, by Theorem 4.9, the sequence $\left\{f_{x_{N}(t)}(x)\right\}_{N=1}^{\infty}$ converges pointwise to $f_{x(t)}(x)$.

The hypotheses of Theorem 4.12 are fulfilled, by doing similar computations to the ones in 4.23 . Hence, $\mathbb{E}\left[x_{N}(t, \omega)\right] \rightarrow \mathbb{E}[x(t, \omega)]$ and $\mathbb{V}\left[x_{N}(t, \omega)\right] \rightarrow$ $\mathbb{V}[x(t, \omega)]$ as $N \rightarrow \infty$.

In Figure 4.3 , we observe that the sequence $\left\{f_{x_{N}(0.3)}(x)\right\}_{N=1}^{\infty}$ seems to converge, for $N=1,2,3,4,5,6$. In Table 4.3, we approximate the expectation and variance of $x(t, \omega)$. 


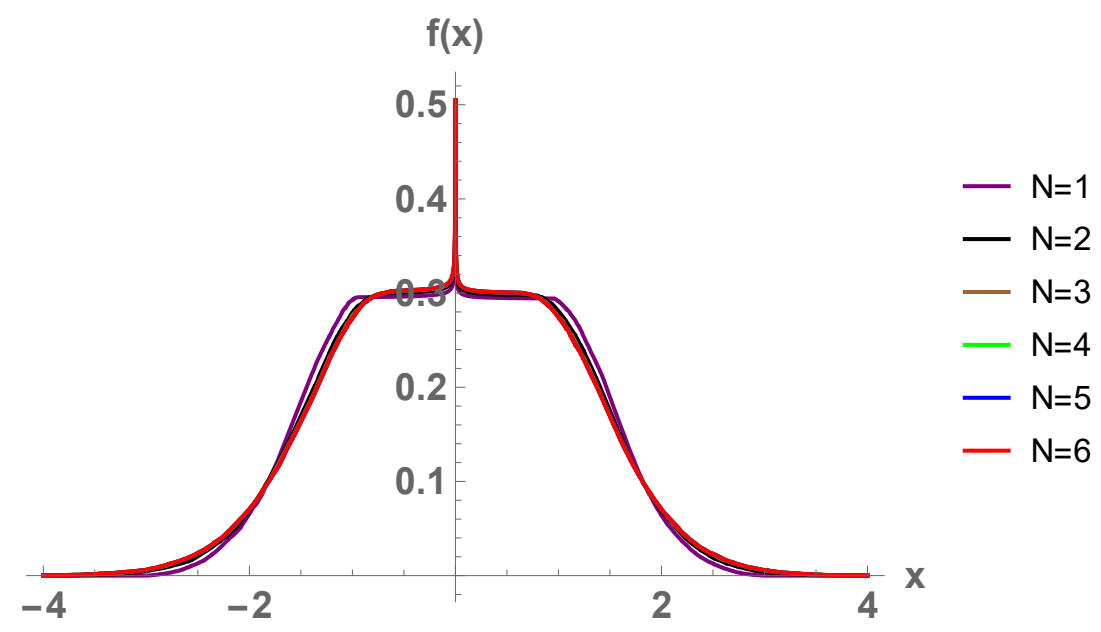

Figure 4.3: Density $f_{x_{N}(0.3)}(x)$ for $N=1,2,3,4,5,6$. Example 4.15

\begin{tabular}{|c|c|c|c|c|c|c|}
\hline$N$ & 1 & 2 & 3 & 4 & 5 & 6 \\
\hline $\mathbb{E}\left[x_{N}(0.3, \omega)\right]$ & 0.0014 & 0.0001 & 0.0001 & 0.0002 & 0.0002 & 0.0002 \\
\hline $\mathbb{V}\left[x_{N}(0.3, \omega)\right]$ & 1.1240 & 1.1914 & 1.2213 & 1.2298 & 1.2299 & 1.2300 \\
\hline
\end{tabular}

Table 4.3: $\mathbb{E}\left[x_{N}(0.3, \omega)\right]$ and $\mathbb{V}\left[x_{N}(0.3, \omega)\right]$ for $N=1,2,3,4,5,6$. Example 4.15 . 


\subsection{Conclusions}

In this chapter, we have analyzed the random non-autonomous Bertalanffy model: $x^{\prime}(t, \omega)=a(t, \omega) x(t, \omega)+b(t, \omega) x(t, \omega)^{\frac{2}{3}}, t \in\left[t_{0}, T\right]$, with initial condition $x\left(t_{0}, \omega\right)=x_{0}(\omega)$. The coefficients are stochastic processes $a(t, \omega)$ and $b(t, \omega)$ and the initial condition is a random variable $x_{0}(\omega)$ in an underlying complete probability space. Via the usual change of variables for solving deterministic Bernoulli differential equations, we have related the random nonautonomous Bertalanffy model with a random non-autonomous linear differential equation, and thus we have obtained a formal solution stochastic process $x(t, \omega)$. Theorem 4.1 says when $x(t, \omega)$ has absolutely continuous sample paths that solve the random Bertalanffy model a.e. Theorem 4.2 gives conditions on the moments of $a$ and $b$ under which $x(t, \omega)$ is a mean square solution. By using existing results on the random non-autonomous linear differential equation, the Random Variable Transformation technique and Karhunen-Loève expansions, we have constructed a sequence of density functions that, under certain assumptions, converge pointwise (Theorems 4.6, 4.7 and 4.9) and uniformly (Theorems 4.10 and 4.11) to the density function of $x(t, \omega), f_{x(t)}(x)$. Results on the approximation of the expectation and the variance of $x(t, \omega)$ have been achieved. Finally, these theoretical findings have been treated numerically in the computer.

\section{Acknowledgements}

This work has been supported by the Spanish Ministerio de Economía y Competitividad grant MTM2017-89664-P. I am grateful to the Department of Differential Equations and Numerical Analysis of the University of Sevilla (Spain), for hosting me during a research stay.

The main results of this chapter have been submitted for publication. This chapter is a national collaboration with the researcher Tomás Caraballo Garrido (Departamento de Ecuaciones Diferenciales y Análisis Numérico, Universidad de Sevilla, Spain). 
Chapter 5

\section{Combining polynomial chaos expansions and the random variable transformation technique to approximate the density function of stochastic problems.}

In this chapter, we deal with computational uncertainty quantification for stochastic models with one random input parameter. The goal of the chapter is twofold: First, to approximate the set of probability density functions of the solution stochastic process, and second, to show the capability of our theoretical findings to deal with some important epidemiological models. The approximations are constructed in terms of a polynomial evaluated at the random input parameter, by means of generalized polynomial chaos expansions and the stochastic Galerkin projection technique. The probability density function of the aforementioned univariate polynomial is computed via the random variable transformation method, by taking into account the domains where the polynomial is strictly monotone. The algebraic/exponential convergence of the Galerkin projections gives rapid convergence of these density functions. The examples are based on fundamental epidemiological models formulated via linear and nonlinear differential and difference equations, where one of the input parameters is assumed to be a random variable. 


\subsection{Introduction}

In the recent contribution [46, RVT and gPC techniques have been successfully combined to study random differential equations. That study focused on the computation of the mean and the variance of the solution stochastic process for random differential equations. In the present chapter, we combine both techniques to go further and to compute reliable approximations to the probability density function of stochastic models. Furthermore, we point out that the proposed method possesses rapid convergence and is able to reach its goal even in the case that no closed-form solution to the stochastic problem is available. This is a distinctive feature of our approach since, as it has been previously indicated, the application of the RVT technique to determine the probability density function of stochastic models usually requires knowing a closedform expression of the solution, see for instance [29, 52, 23, 53, 72, 73, 74, 146]. All these features of our approach will be illustrated through several epidemiological models. At this point it is important to underscore that in the context of epidemiological modeling, knowledge of the probability density function is crucial, since from it, apart from computing predictions (via the mean) and confidence intervals (via the mean and the standard deviation), one can also compute the probability that the proportion of infected individuals lies in a certain interval of interest for health authorities [29, 30, 80] (prevention and control of plagues, identify seasonal patterns of infection, etc.).

Hereinafter, we will work with stochastic models with only one random input parameter. The reason for this restriction is that there is a version of the RVT technique for non-injective transformation mappings on $\mathbb{R}$ with many applications in general, but not on $\mathbb{R}^{s}, s>1$. The RVT method will allow us to compute the exact density function of the Galerkin projections (which are polynomials, usually non-injective), and by taking limits we will approximate the density function of the response process. Since the Galerkin projections converge at algebraic/exponential rate to the solution process, it is expected to have rapid convergence of the sequence of approximating density functions.

From a modeling standpoint, the variability of each parameter (input) produces a variability in the results (output) of the model. However, the effects of the variabilities of the parameters are of different magnitude and can be estimated by using a sensitivity analysis method such as gPC-based Sobol's indices [117, 49, 130, 34]. These indices explain the influence of each random parameter on the response process, by performing a variance-based sensitivity analysis. The variance of the output is a sum of contributions of each input variable and combinations of them. Indeed, the variance of the output is the 
squared-sum of the Galerkin coefficients, so the contribution of the main effect of a parameter is the normalized squared-sum of the coefficients corresponding to basis polynomial functions evaluated only at the aforesaid parameter. By computing Sobol's indices, it is assumed that the parameter that leads to a larger variance of the output has the largest effect on the model prediction. If one is dealing with epidemic models, the parameter with highest gPC-based Sobol's index is the most important to address and control the epidemic, and the other parameters may be taken to be deterministic. Therefore the fact of introducing randomness into only one input parameter may be justified.

The structure of the chapter is the following. In Section 5.2, we will specify the type of stochastic problems under study, we will show the necessary basics of gPC expansions and the RVT technique, and we will combine both methods to approximate the probability density function of the solution stochastic process. In Section 5.3. we will illustrate the theoretical development with several particular examples of continuous and discrete stochastic models in an epidemiological and social setting. Finally, Section 5.4 will draw conclusions.

\subsection{Method}

We consider an initial value differential equation problem with one degree of uncertainty:

or

$$
\left\{\begin{array}{l}
y^{\prime}(t)=F(y(t), t), t \in I \\
y\left(t_{0}\right)=\zeta
\end{array}\right.
$$

$$
\left\{\begin{array}{l}
y^{\prime}(t)=F(y(t), t, \zeta), t \in I, \\
y\left(t_{0}\right)=y_{0},
\end{array}\right.
$$

where $I \subseteq \mathbb{R}$ is an interval, $t_{0} \in I, F$ is a deterministic function, and $\zeta$ is the random input parameter, which can be the initial condition (case (5.1)), or can appear in the differential equation expression (case (5.2), with the initial condition $y_{0}$ being deterministic). The random variable $\zeta$ is assumed to be absolutely continuous (we denote its density function by $f_{\zeta}(\zeta)$ ) with centered absolute moments of any order. At this point, it is important to point out that the largest part of random variables that are used in practice, specially in epidemiological modeling, such as Beta, Gaussian, Gamma, Uniform, Triangular, etc., satisfy these hypotheses. In this setting, we are assuming an underlying complete probability space $(\Omega, \mathcal{F}, \mathbb{P})$, where $\Omega$ is the sample space consisting of outcomes $\omega \in \Omega, \mathcal{F}$ is the $\sigma$-algebra of events and $\mathbb{P}$ is the probability measure. 
The solution $y(t)$ to $(5.1)$ or $(5.2)$ is a stochastic process. We will assume that such a solution exists and is unique, in some stochastic sense (sample path sense, mean square sense, etc., see [125, 129]). Our goal is to compute the probability density function of $y(t), f_{y(t)}(y)$, at each time instant $t \in I$. The density function allows performing uncertainty quantification for $y(t)$, since all the statistical moments and confidence intervals can be derived from it 125] (Chapter 2-3).

Other alternative stochastic systems to (5.1) and (5.2) are possible as well: random partial differential equations, random difference equations, etc., with one degree of uncertainty. For such stochastic systems, the techniques exposed here work analogously.

\subsection{1 gPC Expansions}

Given the random input parameter $\zeta$, consider its associated canonical basis $\mathcal{C}^{p}=\left\{1, \zeta, \zeta^{2}, \ldots, \zeta^{p}\right\}$, for each degree $p \geq 1$. By using a Gram-Schmidt procedure, we orthonormalize this basis in the Hilbert space of random variables with well-defined variance, $\left(\mathrm{L}^{2}(\Omega),\langle\rangle,\right)$, where the inner product is defined as $\langle X, Y\rangle=\mathbb{E}[X Y]$ for $X, Y \in \mathrm{L}^{2}(\Omega)$, being $\mathbb{E}$ the expectation operator with respect to the probability measure $\mathbb{P}$. Let $\Xi^{p}=\left\{\phi_{0}(\zeta), \phi_{1}(\zeta), \ldots, \phi_{p}(\zeta)\right\}$ be the orthonormal basis of $\mathcal{C}^{p}, p \geq 1$, where $\phi_{0}=1$. For example, if $\zeta \sim$ $\operatorname{Normal}(0,1)$, then $\Xi^{p}$ is the set of Hermite polynomials; if $\zeta \sim \operatorname{Uniform}(-1,1)$, then $\Xi^{p}$ is formed by Legendre polynomials, etc. [142, 143]. Notice that, with this approach, we are not restricted to distributions from the Askey scheme [142, Ch. 3], as already remarked in [33, 46]. This point has great interest in practice, since most of the random variables constructed to model real phenomena do not fit the standard distributions, and therefore we are enlarging the applicability of the classical gPC method.

If $Z$ is a random variable in $\mathrm{L}^{2}(\Omega)$ written as a function of $\zeta, Z=r(\zeta)$, then the gPC expansion of $Z$ is $Z=\sum_{i=0}^{\infty} \hat{Z}_{i} \phi_{i}(\zeta)$, where the sum is considered in $\mathrm{L}^{2}(\Omega)$ and the Fourier coefficients $\hat{Z}_{i}$ are given by $\hat{Z}_{i}=\mathbb{E}\left[r(\zeta) \phi_{i}(\zeta)\right]$. Actually, to ensure that $Z=\sum_{i=0}^{\infty} \hat{Z}_{i} \phi_{i}(\zeta)$, we need that the moment problem is uniquely solvable for $\zeta$, see [58, Th. 3.3]. This condition is not so restrictive, as any random variable with finite moment-generating function in a neighborhood of the origin has a uniquely solvable moment problem [58, Th. 3.4].

If we take the solution stochastic process $y(t)$, then we may formally write $y(t)=\sum_{i=0}^{\infty} \hat{y}_{i}(t) \phi_{i}(\zeta)$ in $\mathrm{L}^{2}(\Omega)$, where $\hat{y}_{i}(t)=\mathbb{E}\left[y(t) \phi_{i}(\zeta)\right]$. The Fourier coefficients $\hat{y}_{i}(t)$ cannot be explicitly computed, as $y(t)$ is unknown. We use 
the stochastic Galerkin projection technique [33, 46, 142, 143]: We seek a solution of the form $\tilde{y}^{p}(t)=\sum_{i=0}^{p} \tilde{y}_{i}^{p}(t) \phi_{i}(\zeta)$, i.e., we impose

$$
\sum_{i=0}^{p} \frac{\mathrm{d}}{\mathrm{d} t} \tilde{y}_{i}^{p}(t) \phi_{i}(\zeta)=F\left(\sum_{i=0}^{p} \tilde{y}_{i}^{p}(t) \phi_{i}(\zeta), t\right) \text {. }
$$

The deterministic functions $\tilde{y}_{i}^{p}(t)$ are calculated in two steps: first, we multiply the previous equation by $\phi_{k}(\zeta)$, and we take the expectation operator and use the orthonormality of $\Xi^{p}$ :

$$
\frac{\mathrm{d}}{\mathrm{d} t} \tilde{y}_{k}^{p}(t)=\left\langle F\left(\sum_{i=0}^{p} \tilde{y}_{i}^{p}(t) \phi_{i}(\zeta), t\right), \phi_{k}(\zeta)\right\rangle, k=0, \ldots, p ;
$$

and secondly, we solve a deterministic system of differential equations via standard numerical techniques. Formally, $\lim _{p \rightarrow \infty} \tilde{y}^{p}(t)=y(t)$ in $\mathrm{L}^{2}(\Omega)$. Some general results justify this assertion, see [21, 121]. Moreover, algebraic/exponential convergence usually holds (spectral convergence).

The stochastic Galerkin projection technique and gPC expansions have been profusely used in the literature for uncertainty quantification, see for example [24, 35, 90, 117, 126, 138, 144].

We will show how to compute exactly the probability density function of $\tilde{y}^{p}(t)$, which will be used as an approximation for $f_{y(t)}$.

\subsubsection{RVT Technique}

The RVT technique has been successfully applied to study significant random ordinary differential equations [23, 29, 52], random partial differential equations [23, 53, 72, 73, 74, 146] and random recursive equations 44, that appear in different areas such as Epidemiology, Physics, Engineering, etc. In this chapter, we will use the following version of the RVT technique:

Theorem 5.1 [83, p. 115] Let $X$ be an absolutely continuous random variable with density $f_{X}$ and with support $D_{X}$ contained in an open set $D \subseteq \mathbb{R}$. Let $g$ : $D \rightarrow \mathbb{R}$ be such that $D=\dot{\cup}_{1 \leq i \leq n} D_{i}$ and $g_{i}=\left.g\right|_{D_{i}}$ is injective and $C^{1}\left(D_{i}\right)$ with non-vanishing derivative. Then the random variable $Y=g(X)$ is absolutely continuous, with density function given by

$$
f_{Y}(y)= \begin{cases}\sum_{i: y \in g\left(D_{i}\right)} f_{X}\left(g_{i}^{-1}(y)\right)\left|\frac{\mathrm{d} g_{i}^{-1}(y)}{\mathrm{d} y}\right|, & y \in g(D), \\ 0, & y \notin g(D) .\end{cases}
$$


Although there is a version of this theorem for $\mathbb{R}^{s}, s>1$, its applicability in our context will be reduced to $\mathbb{R}$. For example, when working with transformation mappings $g$ given by polynomials (this will be the case of the Galerkin projections), in dimension one we can find the regions $D_{1}, \ldots, D_{n}$ by equating the derivative $g^{\prime}$ to 0 ; however, polynomials on $\mathbb{R}^{s}, s>1$, is a different matter, as the regions $D_{1}, \ldots, D_{n}$ may be impossible to find or even not exist. This fact motivates that in this first step of our research we consider the case $s=1$, which has specific interest.

\subsubsection{Combining gPC and RVT Technique to Approximate the Density Function}

Fixed $t \in I$ and $p \geq 1$, the Galerkin projection $\tilde{y}^{p}(t)=\sum_{i=0}^{p} \tilde{y}_{i}^{p}(t) \phi_{i}(\zeta)$ is an explicit transformation of the random variable $\zeta$, let us call it $g(\zeta)$. Since $g(\zeta)$ is a polynomial, by solving numerically $g^{\prime}(\zeta)=0$ we can determine the sets $D_{1}, \ldots, D_{n}$ where $g$ is strictly monotone and find $g\left(D_{i}\right)$. At each $\xi \in g\left(D_{i}\right)$, by finding numerically the unique root $\zeta \in D_{i}$ such that $\xi=g(\zeta)$, we compute the inverse of $g_{i}=\left.g\right|_{D_{i}}, h_{i}(\xi):=g_{i}^{-1}(\xi)$. By the RVT technique,

$$
f_{\tilde{y}^{p}(t)}(\xi)=f_{g(\zeta)}(\xi)=\sum_{\substack{i: 1 \leq i \leq n \\ \xi \in g\left(D_{i}\right)}} f_{\zeta}\left(h_{i}(\xi)\right)\left|h_{i}^{\prime}(\xi)\right| .
$$

Since $h_{i}(\xi)$ has been computed numerically for each $\xi$, we do not have $h_{i}^{\prime}$ explicitly. We compute $h_{i}^{\prime}$ as

$$
h_{i}^{\prime}(\xi)=\frac{1}{g^{\prime}\left(h_{i}(\xi)\right)}, \quad \xi \in g\left(D_{i}\right),
$$

instead. Thus,

$$
f_{\tilde{y}^{p}(t)}(\xi)=f_{g(\zeta)}(\xi)=\sum_{\substack{i: 1 \leq i \leq n \\ \xi \in g\left(D_{i}\right)}} f_{\zeta}\left(h_{i}(\xi)\right) \frac{1}{\left|g^{\prime}\left(h_{i}(\xi)\right)\right|} .
$$

Notice that the RVT technique has permitted us to obtain the exact expression for the probability density function of the Galerkin projection $\tilde{y}^{p}(t)$. This exact expression is a distinctive feature of the RVT technique comparing with other stochastic methods (for example, gPC expansions plus Monte Carlo simulation for the Galerkin projections). 
Since $\lim _{p \rightarrow \infty} \tilde{y}^{p}(t)=y(t)$ in $\mathrm{L}^{2}(\Omega)$ at algebraic/exponential rate, we expect to have

$$
\lim _{p \rightarrow \infty} f_{\tilde{y}^{p}(t)}(\xi)=f_{y(t)}(\xi)
$$

with rapid convergence. This procedure works as a computational method to approximate $f_{y(t)}(\xi)$. To the best of our knowledge, there are no available theoretical results that guarantee that (5.4) holds, but only mean square convergence of the Galerkin projections $\tilde{y}^{p}(t)$, instead.

In principle, we know nothing about the type of convergence of the sequence $\left\{f_{\tilde{y}^{p}(t)}\right\}_{p=1}^{\infty}$ to the true density $f_{y(t)}$. The numerical experiments that we will carry out in the forthcoming section, see Example 5.2 for instance, show that uniform convergence may not be expected, as $f_{y(t)}$ might be discontinuous but $f_{\tilde{y}^{p}(t)}$ continuous. Hence, in general, one may expect convergence of $\left\{f_{\tilde{y}^{p}(t)}\right\}_{p=1}^{\infty}$ in some Lebesgue space.

Assume that $\left\{f_{\tilde{y}^{p}(t)}\right\}_{p=1}^{\infty}$ is Cauchy in $\mathrm{L}^{1}(\mathbb{R})$. By the completeness of the Lebesgue spaces, there is a function $f_{t}^{*} \in \mathrm{L}^{1}(\mathbb{R})$ such that $\lim _{p \rightarrow \infty} f_{\tilde{y}^{p}(t)}=f_{t}^{*}$ in $\mathrm{L}^{1}(\mathbb{R})$, for each $t \in I$. Then the limit function $f_{t}^{*}$ is a density function for $y(t)$. Indeed, notice that $f_{t}^{*}$ is a density function, because $f_{t}^{*} \geq 0$ almost everywhere and $\int_{\mathbb{R}} f_{t}^{*}(y) \mathrm{d} y=\lim _{p \rightarrow \infty} \int_{\mathbb{R}} f_{\tilde{y}^{p}(t)}(y) \mathrm{d} y=1$. Let $w(t)$ be a stochastic process such that $w(t) \sim f_{t}^{*}$. We have that $\tilde{y}^{p}(t) \rightarrow w(t)$ in law as $p \rightarrow \infty$, since $\lim _{p \rightarrow \infty} \mathbb{P}\left(\tilde{y}^{p}(t) \leq \xi\right)=\lim _{p \rightarrow \infty} \int_{-\infty}^{\xi} f_{\tilde{y}^{p}(t)}(y) \mathrm{d} y=$ $\int_{-\infty}^{\xi} f_{t}^{*}(y) \mathrm{d} y=\mathbb{P}(w(t) \leq \xi)$. On the other hand, since $\tilde{y}^{p}(t) \rightarrow y(t)$ in $\mathrm{L}^{2}(\Omega)$, in particular in law, as $p \rightarrow \infty$, we conclude that $y(t)$ and $w(t)$ are equal in distribution, therefore $y(t)$ is absolutely continuous and $f_{y(t)}=f_{t}^{*}$. Thus, if the sequence $\left\{f_{\tilde{y}^{p}(t)}\right\}_{p=1}^{\infty}$ is Cauchy, then the limit function must be $f_{y(t)}$.

In practice, we have to be careful with the order of truncation $p$ chosen, since if $p$ is too large, numerical errors may arise, see [21, [121, 62]. This is a disadvantage of gPC expansions. There may be an order of truncation $p_{0}$ such that, for any $p \geq p_{0}$, the results obtained make no sense, therefore the density function may not be computed as accurately as wanted. See the forthcoming Example 5.4. 


\subsection{Numerical Experiments}

In this section, we deal with particular continuous and discrete stochastic models that play an important role in Epidemiology. By using the previous ideas, we will approximate the probability density function of the solution stochastic process to some epidemiological models at different time instants. In the field of Epidemiology, the computation of the probability density function is a primary goal, as the expected number or confidence intervals for infective (predictions), or any other information of interest for health authorities, can be derived from it.

The computations have been carried out in the software Mathematica ${ }^{\circledR}$. To compute the roots of $g^{\prime}(\zeta)=0$ and $\xi=g(\zeta)$, the built-in function NSolve has been utilized. The solution to deterministic systems of differential equations has been solved by means of the standard NDSolve routine, with automatic method, step size, etc.

When dealing with real data, we will compute deterministic estimates for the model parameters with a least squares fitting procedure (FindFit builtin function). Then we will introduce a small perturbation into one of the parameters (with mean value being the deterministic estimate calculated) and we will apply the methodology previously exposed. Introducing only a small amount of randomness into the model from deterministic estimates allows a more faithful representation of the time evolution of the population, see for example [33, Example 5], [13, 24, 117, 126, 127]. In this article, as we are interested in testing our methodology on approximating the density function of the model output, but not in estimating probability distributions for the input parameters, we do not deal with inverse parameter estimation, see, for example, references [143] (pp. 95-99), [96, 97], related with Bayesian inference and gPC expansions.

In the examples, we will see that it suffices to take a small order $p$ of basis to get accurate approximations of the probability density function. The optimal $p$ is that from which the subsequent probability density functions constructed for larger orders than $p$ are virtually the same. In previous contributions dealing with approximations of the mean and variance statistics in the setting of continuous and discrete epidemic models (SIS, SIR, etc. models), see [35. 24, it was shown numerically that, although for $p=1$ the approximations are not good, for $p=2$ and $p=3$ very similar results are produced.

In Example 5.2, the approximations of the probability density functions constructed by combining gPC expansions and the RVT technique will be com- 
pared with the exact density function. For Example 5.3, Example 5.4, and Example 5.5, we will compare our approximations against a Kernel density estimation from Monte Carlo simulations sampled from the stochastic problem directly, with the built-in function SmoothKernelDistribution of Mathematica ${ }^{\circledR}$. We will check that Kernel density estimations do not approximate well when the sought density function is not smooth (see the documentation on the built-in function SmoothKernelDistribution, or the theoretical reference [119]), while our method substantiated on the RVT technique is certainly able to identify discontinuities and peaks, i.e., the exact shape of the target density function. Moreover, gPC-based methods converge much faster than Monte Carlo simulation, even when there is only one random input coefficient, see the discussions from previous contributions [33, 142, 143]. Indeed, Monte Carlo simulation converges relatively slow (for example, the mean value converges at rate $1 / \sqrt{m}$, where $m$ is the number of realizations), whereas gPC expansions converge at spectral rate.

When no explicit form of the solution is available, representations of it via infinite expansions (gPC expansions, random power series, Karhunen-Loève expansions, etc.) or discretizations from numerical methods are usually required. By truncating these infinite expansions or from the discretizations, one constructs a sequence of approximating processes, whose exact density functions (computed via the RVT technique) form a sequence of approximating density functions that converge rapidly [17, 19, 45, 57]. In terms of rapidity and accuracy, this sort of approximations are preferable to Monte Carlo simulation and Kernel density estimations.

In Remark 5.6 from Example 5.5, we will show how gPC-based Sobol's indices (see Section 5.1) may be used to determine the parameter whose variability has the most important effects and therefore justify the fact of introducing uncertainty into one input parameter only, in the context of a social model for addictive behavior.

Example 5.2 In this example, we consider the simplest continuous epidemiological model to describe the growth of a population of bacteria with no limitation of resources and no antibiotics (predators). The model has demonstrated to be still useful to describe this population growth in presence of competitors during the initial stage. Proposed by Thomas Malthus in 1798 in his essay [95], this model is considered as the first law of population dynamics and is usually referred to as exponential, first-order kinetics, or Malthusian model [10, Ch. 1], [102]. With the aim of illustrating the main ideas exhibited in the previous section, next we deal with the following particular case of the 
Malthusian model which has been addressed in [142, pp. 69-70], [143, p. 10]:

$$
\left\{\begin{array}{l}
y^{\prime}(t)=\zeta y(t), \\
y(0)=3,
\end{array}\right.
$$

where $\zeta \sim \operatorname{Beta}(2,1)$. This initial value problem may correspond to a bacteria with initial population 3 million of individuals and whose relative instantaneous growth rate fluctuates randomly in the unit interval. For this stochastic model, the solution stochastic process is well-known: $y(t)=3 \mathrm{e}^{\zeta t}$. By means of the RVT technique, the exact probability density function of $y(t)$ can be calculated:

$$
f_{y(t)}(y)=f_{\zeta}\left(\frac{1}{t} \log \left(\frac{y}{3}\right)\right) \frac{1}{y|t|}, \quad y>0, \quad t \neq 0 .
$$

Figure 5.1 plots $f_{y(t)}$ for $t=0.5$ and $t=1$.
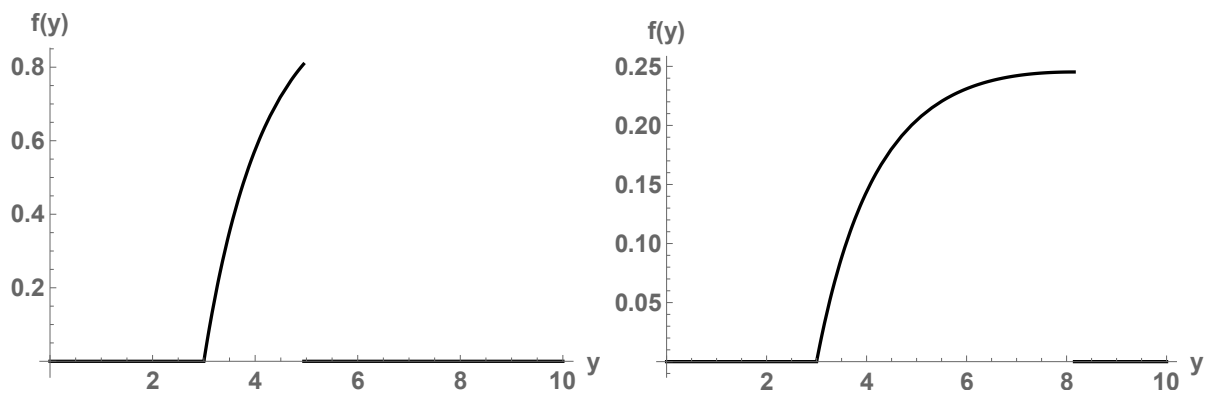

Figure 5.1: Exact graph of $f_{y(t)}(y)$ for $t=0.5$ (first) and $t=1$ (second) in Example 5.2

Let us test our methodology. We use our computational method to approximate the probability density function of $y(t)$ at the times $t=0.5$ and $t=1$. In Figure 5.2 we show the corresponding results. We observe that, as the order of basis $p$ increases, the sequence of density functions $f_{\tilde{y}^{p}(t)}(\xi)$ of the Galerkin projections $\tilde{y}^{p}(t)$ tends to $f_{y(t)}(\xi)$. Based on observation of Figure 5.2, this convergence seems to hold in $\mathrm{L}^{q}(\mathbb{R}), 1 \leq q<\infty$, but not uniformly on $\mathbb{R}$, since $f_{\tilde{y}^{p}(t)}(\xi)$ seems continuous for $p>1$. Table 5.1 shows the $\mathrm{L}^{1}(\mathbb{R})$ error for each order $p$ :

$$
\left\|f_{y(t)}-f_{\tilde{y}^{p}(t)}\right\|_{\mathrm{L}^{1}(\mathbb{R})}=\int_{\mathbb{R}}\left|f_{y(t)}(\xi)-f_{\tilde{y}^{p}(t)}(\xi)\right| \mathrm{d} \xi .
$$


This integral has been calculated in Mathematica ${ }^{\circledR}$ with the NIntegrate routine. Observe that the error decreases to 0 very fast (exponentially), although convergence deteriorates when time $t$ increases.

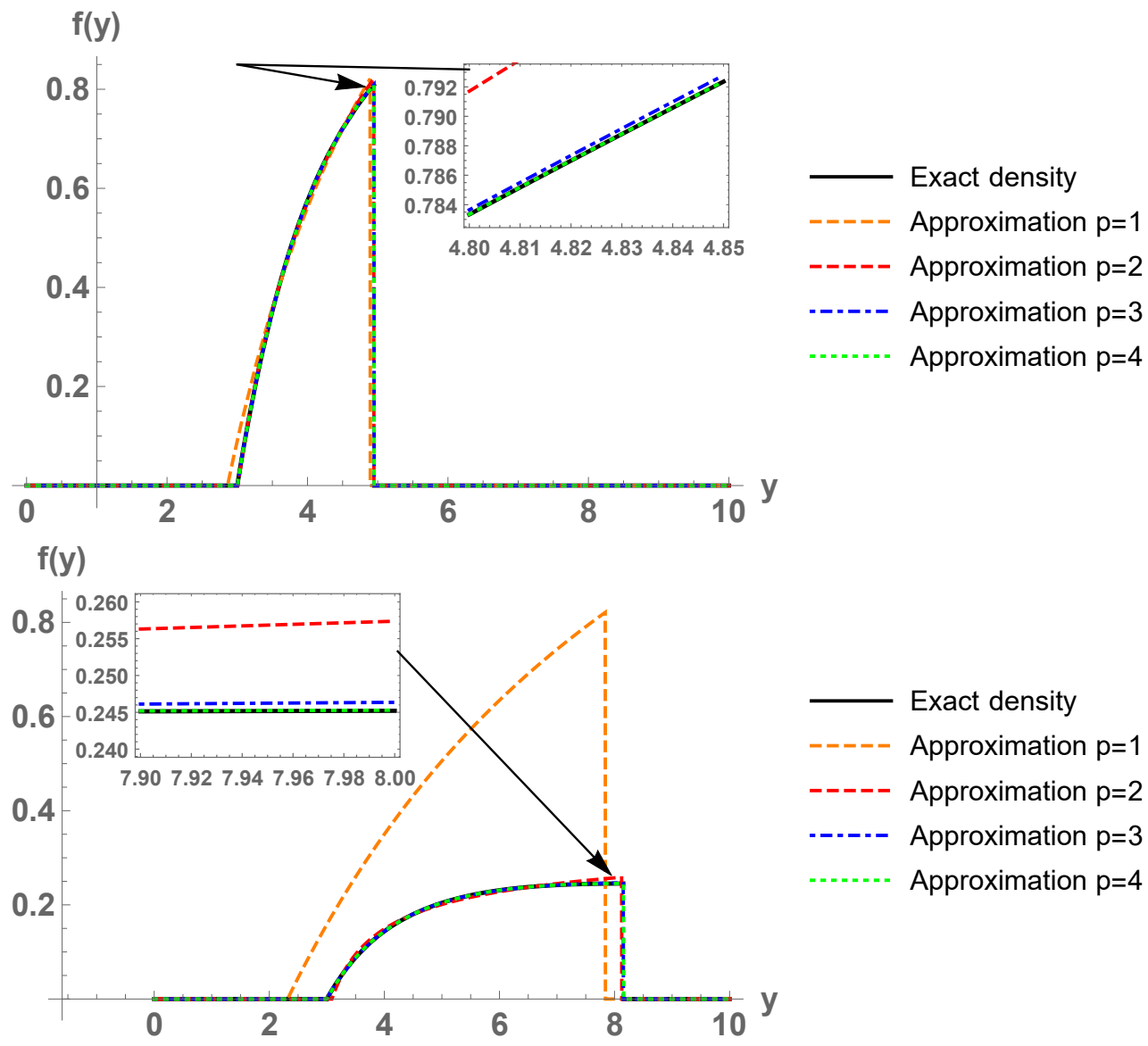

Figure 5.2: Approximation of $f_{y(t)}(y)$ via generalized polynomial chaos (gPC) and random variable transformation (RVT) for $t=0.5$ (first plot) and $t=1$ (second plot) in Example 5.2 Observe the rapid convergence to the exact density function $f_{y(t)}(y)$ as $p$ grows.

Example 5.3 In this example, we deal with a stochastic model applied to real laboratory data of bacteria growth [127]. We consider a species of bacteria from a laboratory experiment: Rhodobacter capsulatus (R. capsulatus). Direct cell counts were made every two or three days until a stationary phase was 


\begin{tabular}{|c|c|c|}
\hline & $t=0.5$ & $t=1$ \\
\hline$p=1$ & 0.0776146 & 1.81093 \\
\hline$p=2$ & 0.00599041 & 0.0304913 \\
\hline$p=3$ & 0.000230145 & 0.00181233 \\
\hline$p=4$ & $7.06091 \cdot 10^{-6}$ & 0.000109031 \\
\hline$p=5$ & $1.71501 \cdot 10^{-7}$ & $5.29540 \cdot 10^{-6}$ \\
\hline$p=6$ & $1.00343 \cdot 10^{-8}$ & $2.15623 \cdot 10^{-7}$ \\
\hline
\end{tabular}

Table 5.1: Error $\left\|f_{y(t)}-f_{\tilde{y}^{p}(t)}\right\|_{\mathrm{L}^{1}(\mathbb{R})}$ for $t=0.5$ and $t=1$, and $p=1,2,3,4,5,6$, in Example 5.2 Note the rapid convergence (exponential) to the exact density function $f_{y(t)}$ as $p$ increases.

achieved. For more information regarding the experiment, we refer the reader to [127.

Table 5.2 shows the population sizes of $R$. capsulatus under infrared lightning conditions in different mediums. The number of cells $/ \mathrm{mL}$ has been rescaled by dividing by $10^{6}$. Figure 5.3 plots the cell counts from Table 5.2.

\begin{tabular}{cc} 
Time $($ days $)$ & Population $\left(\right.$ cells $/ \mathrm{mL}$, scale $\left.10^{6}\right)$ \\
\hline 0 & 0.583 \\
2 & 0.635 \\
4 & 1.08 \\
7 & 3.20 \\
9 & 5.23 \\
11 & 5.28 \\
14 & 5.30
\end{tabular}

Table 5.2: Bacteria population sizes of R. capsulatus [127, Example 5.3 .

In [127, the authors used the Malthusian model to describe the bacteria growth at the first 9 days (before the stationary phase), while a logistic equation model was applied up to day 14. In this example, we suppose that the growth rate $y^{\prime}(t)$ is not proportional to the total number of individuals $y(t)$, but to the total number of interactions, $y(t)^{2}$, via a parameter $r$. Moreover, we take into account the competition for the limited resources with a carrying capacity $K>0$ (limit population under no death assumption), and also the beginning of the bacteria decline phase via a death parameter $\delta>0$. The parameters $r, K$, and $\delta$ are assumed to be deterministic, while the initial 


\section{$R$ capsulatus}

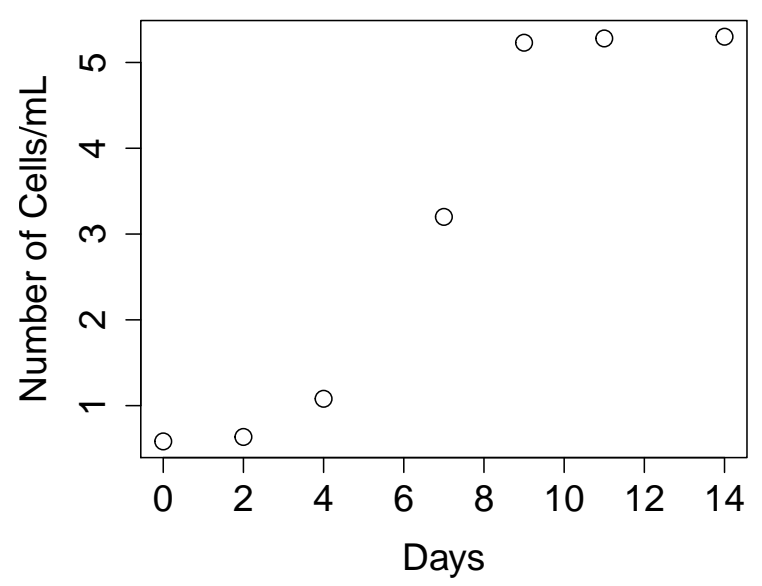

Figure 5.3: Population size of Rhodobacter capsulatus in Example 5.3

condition $\zeta$ is taken as a random variable:

$$
\left\{\begin{array}{l}
y^{\prime}(t)=r y(t)^{2}\left(1-\frac{y(t)}{K}\right)-\delta y(t), \\
y(0)=\zeta
\end{array}\right.
$$

This equation (5.5) corresponds to the simplified Fitzhugh-Nagumo model [36, p. 2].

A least squares fitting gives the deterministic estimates $\hat{r}=0.439310, \hat{\zeta}=$ $0.557972, \hat{K}=5.60822$, and $\hat{\delta}=0.126042$, with a very small residual squared error (smaller than the exponential growth model and the logistic equation presented in [127]). To randomize the initial condition $\zeta$, we consider a distribution centered at $\hat{\zeta}: \zeta \sim \operatorname{Uniform}(0.497972,0.617972)$. The introduction of randomness into the initial condition requires the search of suitable techniques to perform uncertainty quantification. Via gPC expansions and the stochastic Galerkin projection technique with order of basis $p=4$ (this is enough to achieve convergence), pointwise estimates via $\mathbb{E}[y(t)]$, and a confidence interval with the rule $\mathbb{E}[y(t)] \pm \sqrt{\mathbb{V}[y(t)]}$, can be constructed, see Figure 5.4. Observe that the actual data belong to the confidence interval and that the solid line reflects properly the growth of the bacteria population. Notice also that the confidence interval is wider in the middle of the growth phase, while 
smaller uncertainty seems to occur near the initial condition and the carrying capacity.

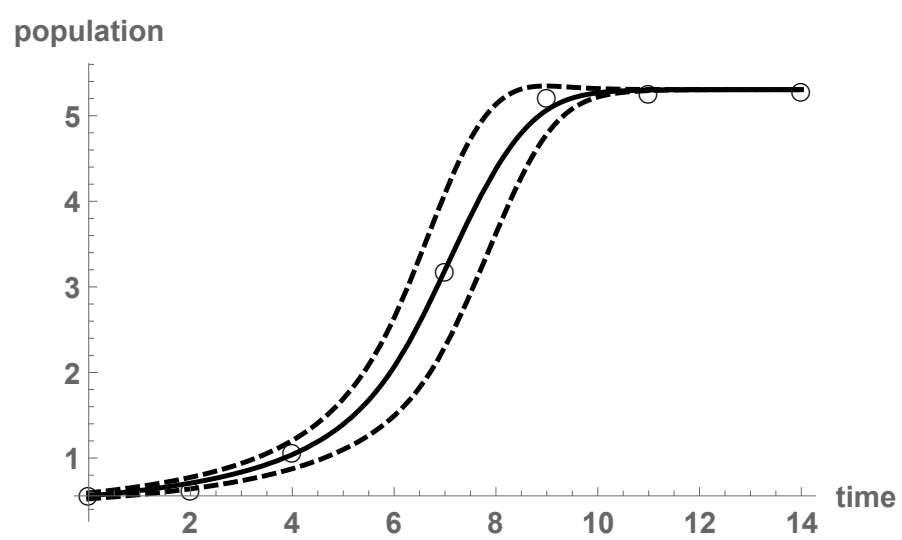

Figure 5.4: Model for the $R$. capsulatus population with $p=4$ in Example 5.3 The circles represent the actual population size, the solid line shows the estimates via $\mathbb{E}[y(t)]$, and the dashed lines reflect the confidence interval.

By using our previous exposition on the combination of the RVT method and gPC expansions, we are even able to determine the probability density function of $y(t)$. In Figure 5.5, we plot the approximations for $p=1,2,3,4$. The similarity of the approximating density functions shows that convergence has been achieved. Our results have been compared with a Kernel density estimation with the built-in function SmoothKernelDistribution. The densities constructed via gPC expansions show that, possibly, the limit density function $f_{y(t)}(y)$ has two jump discontinuities, therefore the Kernel density estimation does not approximate well those discontinuities and draws tails.

Table 5.3 shows consecutive differences for orders of truncation $p$ and $p+1$, $p=1,2,3$ :

$$
\left\|f_{\tilde{y}^{p+1}(t)}-f_{\tilde{y}^{p}(t)}\right\|_{\mathrm{L}^{1}(\mathbb{R})}=\int_{\mathbb{R}}\left|f_{\tilde{y}^{p+1}(t)}(\xi)-f_{\tilde{y}^{p}(t)}(\xi)\right| \mathrm{d} \xi .
$$

Notice that the error decreases to 0 very fast.

Example 5.4 We deal with uncertainty quantification for the following discrete stochastic system [21] (Section 4):

$$
\left\{\begin{array}{l}
y(m+1)=\zeta y(m)(100-y(m)), \\
y(0)=3
\end{array}\right.
$$




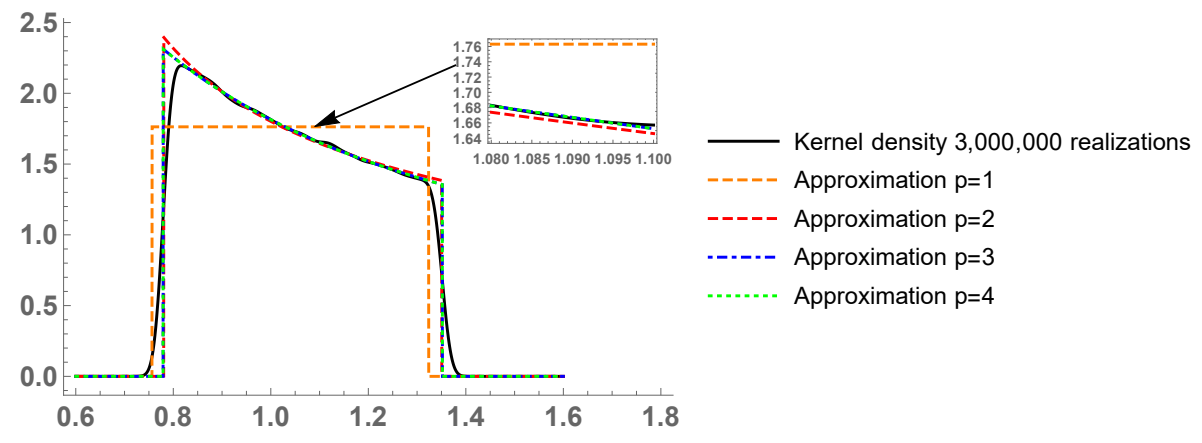

Figure 5.5: Approximation of $f_{y(t)}(y)$ via gPC and RVT for $t=4$ in Example 5.3 . Comparison with a Kernel density estimation.

\begin{tabular}{|c|c|}
\hline & $t=4$ \\
\hline$p=1-p=2$ & 0.202513 \\
\hline$p=2-p=3$ & 0.0105528 \\
\hline$p=3-p=4$ & $3.52486 \cdot 10^{-7}$ \\
\hline
\end{tabular}

Table 5.3: Consecutive difference $\left\|f_{\tilde{y}^{p+1}(t)}-f_{\tilde{y}^{p}(t)}\right\|_{\mathrm{L}^{1}(\mathbb{R})}$ for $t=4$, and $p=1,2,3$, in Example 5.3 Notice the rapid convergence of the consecutive differences to 0 as $p$ grows. 
where $\zeta \sim \operatorname{Triangular}(0.02,0.03)$. In the epidemiological context, if $z(m)$ and $y(m)$ denote, respectively, the number of susceptible and infected individuals in a population of one hundred individuals at the period $m=0,1,2, \ldots$ (e.g., months), the above discrete system describes the dynamics for the number of infected individuals over the time (in months). The parameter $\xi$ can be interpreted as the contagious rate at which a susceptible becomes infected. More precisely, by considering that the product defining the right-hand side of the difference equation gives the number of encounters between susceptible individuals $(z(m)=100-y(m))$ and infected individuals $(y(m))$, then the parameter $\xi$ can be interpreted as the probability that such encounters be successful (i.e., the disease spreading). Since the value of this probability depends upon complex factors such as immunity of each individual of the population, weather, etc., whose nature is unpredictable, it is more realistic to treat $\xi$ as random variable instead of being a deterministic number. As previously indicated and for illustrative purposes only, we will assume that $\xi$ lies in the interval $[0.02,0.03]$ according to a triangular distribution. As a consequence, at each time step $m$, the solution $y(m)$ is a random variable. The Galerkin projection takes the form $\tilde{y}^{p}(m)=\sum_{i=0}^{p} \tilde{y}_{i}^{p}(m) \phi_{i}(\zeta)$, where the deterministic coefficients $\tilde{y}_{i}^{p}(m)$ satisfy a difference equation.

The stochastic Galerkin projection technique has not been applied to discrete models with the same emphasis as with continuous models. In our recent contributions [24, 21, the applications of gPC to discrete dynamical systems have been analyzed. For a large time $m$, the explicit expression of $y(m)$ cannot be obtained or is too complex, so the application of the transformation method is inviable. Thus, one needs appropriate methods to perform uncertainty quantification for $y(m)$. We think that the combination of the RVT technique and $\mathrm{gPC}$ expansions is a good approach to try uncertainty quantification for $y(m)$. The computation of the probability density function of $y(m)$ is a step beyond the numerical experiment performed in [21, Section 4], as [21] only considered the approximation of the expectation and variance statistics.

We approximate the probability density function $f_{y(m)}(y)$ at $m=30$. In Figure 5.6, we show the results. From $p \geq 5$, catastrophic numerical errors invalidate the results. Thus, we are restricted to $p \leq 4$, and we cannot approximate the density function as accurately as desired. This illustrates the main drawback of our computational method. Our results have been compared with a Kernel density estimation with the built-in function SmoothKernelDistribution. Notice that, since the sought density function $f_{y(m)}$ seems not smooth (there may be three peaks because of the triangular 


\begin{tabular}{|c|c|c|c|c|c|c|c|}
\hline Year & 1995 & 1997 & 2001 & 2003 & 2006 & 2011 & 2014 \\
\hline$j$ & 0 & 2 & 6 & 8 & 11 & 16 & 19 \\
\hline$S_{j}$ & 0.5298 & 0.5514 & 0.5783 & 0.6244 & 0.6467 & 0.6863 & 0.6957 \\
\hline
\end{tabular}

Table 5.4: Percentage of nonsmokers Spanish men aged over 16 years old during the period 1995-2014. Source 103 .

form), the Kernel density estimation might not approximate well $f_{y(m)}$ near the peaks.

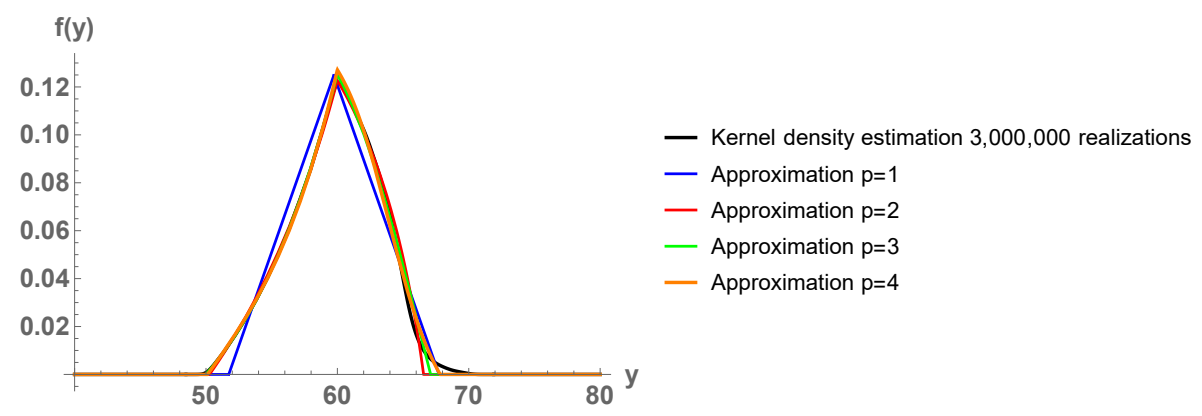

Figure 5.6: Approximation of $f_{y(m)}(y)$ via gPC and RVT for $m=30$ in Example 5.4 Comparison with a Kernel density estimation. From $p \geq 5$, catastrophic numerical errors invalidate the results, so we are restricted to $p \leq 4$.

Example 5.5 In this example, we deal with a social model for the addictive behavior of smoking. We use the data from our recent contribution [24] (Table 1), with original source the National Spanish Health Survey [103]. In Table 5.4 , we show the percentage of nonsmokers Spanish men aged over 16 years old during the period 1995-2014, denoted by $S_{j}, j \in\{0,2,6,8,11,16,19\}$.

As in [24], we use a discrete SIS model:

$$
\begin{aligned}
S(m+1) & =S(m)-b S(m) I(m)+a I(m), \\
I(m+1) & =I(m)+b S(m) I(m)-a I(m) .
\end{aligned}
$$

SIS-type models are useful to describe the spread of diseases (smoking) whose infection does not confer immunity [30, 102, 67]. In the model formulation, $a$ is the proportion of infective (smokers) that leave this compartment (people giving up tobacco), and $b$ is the proportion of contacts between susceptible and 
infective (non-smokers and smokers) that give rise to a new infected individual (smoker). We are assuming homogeneous mixing, i.e., all individuals are equally likely to contact any other individual. We are also supposing a constant total population $N=S(m)+I(m)$. Since our data reflects percentages, we take $N=1$.

From $I(m)=N-S(m)$, we can get rid of the second equation and work with the first one:

$$
S(m+1)=a N+(1-b N-a) S(m)+b S(m)^{2} .
$$

In our usual notation, $y(m)=S(m)$. By using a least squares fitting procedure, the best deterministic estimates for $a$ and $b$ are $\hat{a}=0.0378749$ and $\hat{b}=0.0225118$. We introduce randomness into $a$, with probability distribution Normal(0.0378749, 0.000025). The infection parameter $b$ is constant: $b=0.0225118$.

By using a gPC approach with $p=5$, we approximate the expectation and variance of susceptible individuals $y(m)$. Figure 5.7 plots the results. We observe that the proposed SIS model captures the uncertainty of the smoking habit.

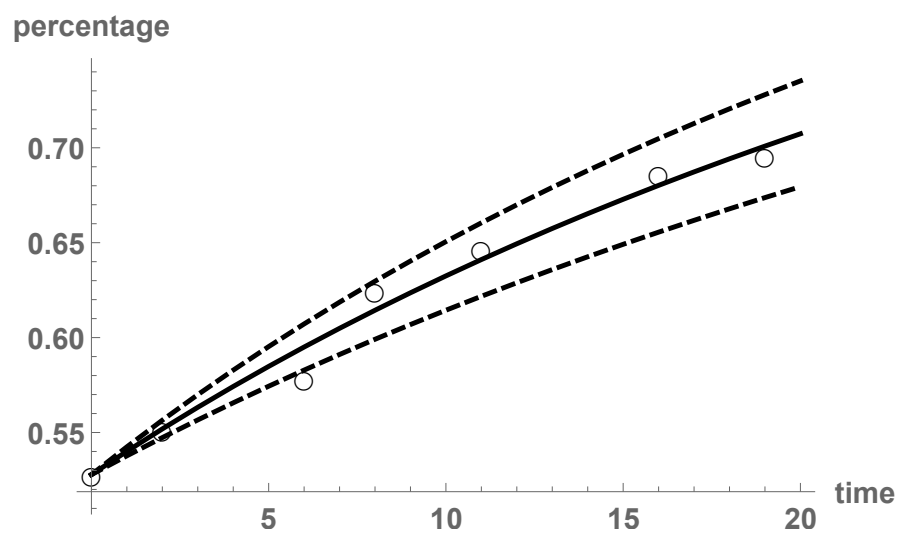

Figure 5.7: Model for the percentage of nonsmokers Spanish men aged over 16 years old during the period 1995-2014 in Example 5.5 The circles represent the actual data, the solid line shows the estimates via $\mathbb{E}[y(m)]$, and the dashed lines reflect the confidence interval.

By combining the RVT technique and gPC expansions, we can go a step further and compute the probability density function of $y(m)$ at times $m=15$ and 
$m=18$, see Figure 5.8. With orders of basis $p=5$, the results agree with a Kernel density estimation with the function SmoothKernelDistribution. In this case, since the density function of $y(m)$ seems smooth (with no peaks), the Kernel density estimation is correct.

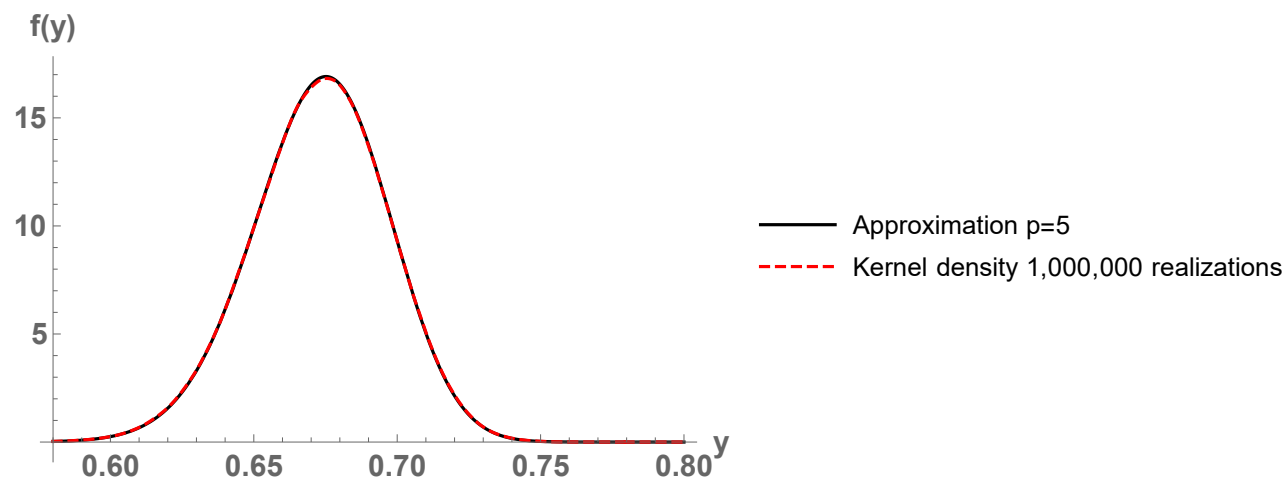

$f(y)$

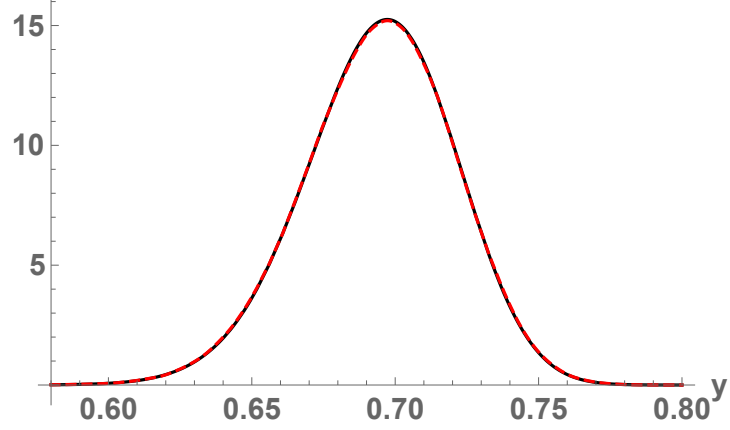

Figure 5.8: Approximation of $f_{y(m)}(y)$ via gPC and RVT for $m=15$ (first plot) and $m=18$ (second plot) in Example 5.5. Observe the rapid convergence to the exact density function $f_{y(m)}(y)$. The results agree with a Kernel density estimation.

Remark 5.6 As we commented, by using a least squares fitting procedure, the best deterministic estimates for $a$ and $b$ are $\hat{a}=0.0378749$ and $\hat{b}=$ 0.0225118. In order to take into account the inherent uncertainty associated to the modeling, we introduce randomness into both parameters: We set $a \sim \operatorname{Normal}(0.0378749,0.000025)$ and $b \sim \operatorname{Uniform}(0.0175118,0.0275118)$. We perform a stochastic Galerkin projection technique with order $p=5$ for both canonical bases. We plot the output of the model in Figure 5.9. 


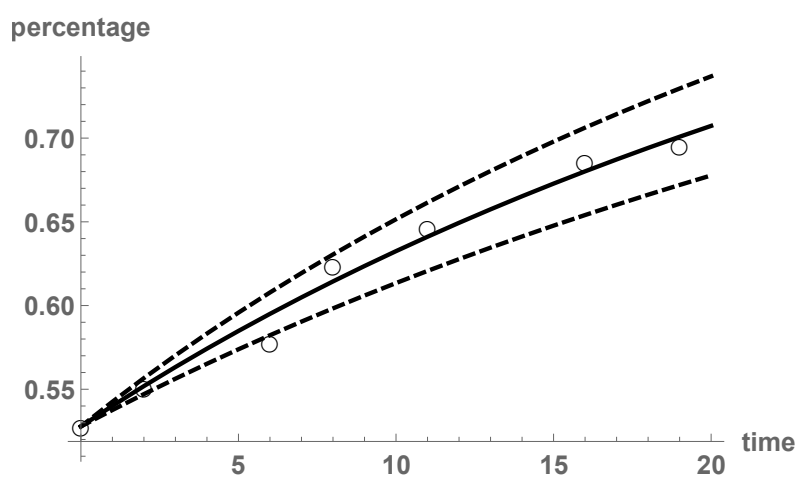

Figure 5.9: Model for the percentage of nonsmokers Spanish men aged over 16 years old during the period 1995-2014 in Remark 5.6 of Example 5.5, with two random input parameters. The circles represent the actual data, the solid line shows the estimates via $\mathbb{E}[y(m)]$, and the dashed lines reflect the confidence interval.

Notice that the outputs from Figures 5.7 and 5.9 are very similar, indicating that parameter $a$ may have the main effect on the model predictions. Indeed, let us check this fact via the gPC-based Sobol's indices. Figure 5.10 depicts the Sobol's indices for $a$ and $b$. Observe that the main effect on the output variance is due to parameter $a$. Thus, the methodology of considering $b=$ $\hat{b}$ constant and putting randomness into $a$ only, as done in Example 5.5 to compute probability density functions via the RVT technique, is justified.

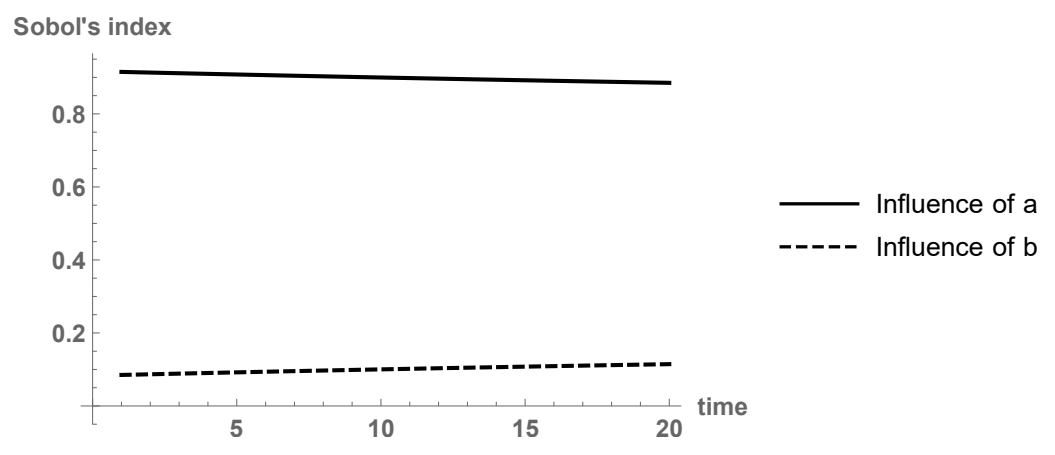

Figure 5.10: Influence of the parameters $a$ and $b$ in the nonsmokers model prediction, Remark 5.6 from Example 5.5 
From an epidemic point of view, people giving up tobacco (parameter $a$ ) is the main reason of the augment of nonsmokers from years 1995 to 2014. Hence, the best policy to address the smoking epidemic is to accomplish strategies that help smokers to give up this addictive behavior.

\subsection{Conclusions}

In this chapter we have approximated the probability density function for differential and difference equations with one random input parameter, by combining the transformation method and generalized polynomial chaos expansions. The method consists in computing the exact density function of the stochastic Galerkin projections, via the RVT technique, for a sufficiently large order of truncation. The optimal order of truncation is that from which the subsequent probability density functions of the Galerkin projections are virtually the same. The sequence of these approximating density functions converges rapidly due to the spectral mean square convergence of the Galerkin projections. Several numerical examples, dealing with both continuous and discrete random dynamical systems in the setting of epidemiological modeling, have illustrated the theoretical ideas. Our approach is restricted to one random input coefficient, since the transformation method for non-injective maps only works on dimension one. Moreover, from a modeling standpoint, one may include uncertainty only into the parameter with largest gPC-based Sobol's index. If we are dealing with an epidemic model, this parameter is the most important to address, control and prevent the epidemic.

To the best of our knowledge, this chapter is the first contribution in which the RVT technique has been combined with gPC expansions to determine the probability density function of stochastic models and go beyond the computation of the expectation and variance, which has been the usual goal in previous contributions dealing with gPC expansions. Moreover, our novelty also relies on applying our methodology to epidemiological models based on continuous and discrete stochastic systems. Being able to quantify the uncertainty associated to epidemics is essential to find the optimal prevention policies.

Part of future research may be devoted to a deeper mathematical analysis of conditions under which the approximating density functions indeed converge pointwise or in some Lebesgue space, so that our computational method would be justified mathematically. 


\section{Acknowledgements}

This work has been supported by the Spanish Ministerio de Economía y Competitividad grant MTM2017-89664-P.

The main results of this chapter have been published in [14. This chapter is an international collaboration with the researcher Benito M. Chen-Charpentier (Department of Mathematics, University of Texas at Austin, USA). 


\section{Chapter 6}

\section{Uncertainty quantification for random parabolic equations via the approximation of the probability density function}

This chapter deals with the randomized heat equation defined on a general bounded interval $\left[L_{1}, L_{2}\right]$ and with non-homogeneous Dirichlet boundary conditions. The solution is a stochastic process that can be related, via changes of variable, with the solution stochastic process of the random heat equation defined on $[0,1]$ with homogeneous Dirichlet boundary conditions. Results in the existing literature establish conditions under which the probability density function of the solution process to the random heat equation on $[0,1]$ with homogeneous boundary conditions can be approximated. Via the changes of variable and the Random Variable Transformation technique, we set mild conditions under which the probability density function of the solution process to the random heat equation on a general bounded interval $\left[L_{1}, L_{2}\right]$ and with non-homogeneous boundary conditions can be approximated uniformly or pointwise. Furthermore, we provide sufficient conditions in order that the expectation and the variance of the solution stochastic process can be computed from the proposed approximations of the probability density function. Numerical examples are performed in the case that the initial condition process has a certain Karhunen-Loève expansion, being Gaussian and non-Gaussian. 


\subsection{Introduction and motivation}

It is well-known that the heat equation plays a key role to describe mathematically diffusion processes. Due to heterogeneity and impurities in materials and errors in the temperature measurements, many authors have proposed to treat the diffusion coefficient, initial and/or boundary conditions in the heat equation as random variables and/or stochastic processes rather than deterministic constants and functions, respectively. This approach leads to stochastic and random heat equation formulation [124, pp. 96-97]. In the former case, the stochastic heat differential equation is forced by an irregular stochastic process such as a White Noise process [70, Ch. 4]. These kind of equations are typically written in terms of stochastic differentials and interpreted as Itô or Stratonovich integrals. Special stochastic calculus is usually applied to obtain exact or approximate solutions to this class of differential equations [70, 82, 106].

In [145], a new stochastic analysis for steady and transient one-dimensional heat conduction problem based on the homogenization approach is proposed. Thermal conductivity is assumed to be a random field depending on a finite number random variables. Both mean and variance of stochastic solutions are obtained analytically for the field consisting of independent identically distributed random variables. In [37, the stochastic temperature field is analyzed by considering the annular disc to be multi-layered with spatially constant material properties and spatially constant but random heat transfer coefficients in each layer. A type of integral transform method together with a perturbation technique are employed in order to obtain the analytical solutions for the statistics (mean and variance) of the temperature. Another fruitful approach to deal with different formulations of the random heat equation is the Mean Square Calculus [125, Ch. 4]. In [26, an analytic-numerical mean square solution of the random diffusion model in an infinite medium is constructed by applying the random exponential Fourier integral transform. A complementary analysis, based on random trigonometric Fourier integral transforms, to solve random partial differential heat problems with non-homogeneous boundary value conditions has been presented in [27. In these two latter contributions, reliable approximations for the mean and the variance of the solution stochastic process are provided. Likewise asymptotic-preserving methods for random hyperbolic, transport equations and radiative heat transfer equations with random inputs and diffusive scalings have been recently studied using generalized polynomial chaos based stochastic Galerkin method [75, 76]. The probabilistic information to the solution stochastic process of the random heat equation in all the aforementioned contributions focus on first statistical moments like the 
mean and the variance functions. Nevertheless, a more ambitious target is to determine exact or reliable approximations of the first probability density function of the solution stochastic process, since from it all one-dimensional statistical moments can be obtained, if they exist. In particular, the mean and the variance can be straightforwardly derived via integration of the first probability density function [125, Ch. 3]. In the context of random partial differential equations, some recent contributions addressing this significant problem include, for example, [53, 72, 73, 146] (see also references therein). From a general standpoint, this chapter is aimed to contribute further the study of methods to determine rigorous approximations of the first probability density function of random partial differential equations focusing on the random heat equation. It is important to point out that the subsequent analysis is based upon our previous contribution [16].

In [16], we have studied the randomized heat equation on the spatial domain $[0,1]$ with homogeneous boundary conditions and assuming that the diffusion coefficient is a positive random variable and that the initial condition is a stochastic process. In a first step, the solution stochastic process of that stochastic problem was rigorously constructed using two different approaches, namely, the Sample Calculus [125, Appendix I] and the Mean Square Calculus [125. Ch. 4]. The second, and main step, consisted of constructing approximations of the probability density function of the solution by combining the Random Variable Transformation technique and the Karhunen-Loève expansion. Several results providing sufficient conditions to guarantee the pointwise and uniform convergence of these approximations were established. The aim of this contribution is to extend the study to the case where boundary conditions are random variables and assuming that the problem is stated on an arbitrary interval, say $\left[L_{1}, L_{2}\right]$. Since this extension depends heavily on some results established in [16], for the sake of completeness down below we summarize them.

\subsection{Preliminaries}

\subsubsection{Preliminaries on the randomized heat equation on $[0,1]$ with homogeneous Dirichlet boundary conditions}

Reference [16] provides the necessary results on the approximation of the probability density function of the randomized heat equation on the spatial domain $[0,1]$ with homogeneous boundary conditions. The main goal of this contribu- 
tion is to extend these results to the randomized heat equation on a general interval $\left[L_{1}, L_{2}\right]$ with random boundary conditions.

The heat equation on the spatial domain $[0,1]$ with homogeneous Dirichlet boundary conditions has the form

$$
\left\{\begin{aligned}
v_{t} & =\beta^{2} v_{x x}, & & 0<x<1, t>0 \\
v(0, t) & =v(1, t)=0, & & t \geq 0 \\
v(x, 0) & =\psi(x), & & 0 \leq x \leq 1
\end{aligned}\right.
$$

We consider 6.1 in a random setting. Let $(\Omega, \mathcal{F}, \mathbb{P})$ be a complete probability space, where $\Omega$ is the sample space, which consists of outcomes that will be denoted by $\omega, \mathcal{F}$ is a $\sigma$-algebra of events and $\mathbb{P}$ is the probability measure. The diffusion coefficient is considered as a positive random variable $\beta^{2}(\omega)$ and the initial condition is a stochastic process $\psi=\{\psi(x)(\omega): x \in[0,1], \omega \in \Omega\}$ in the underlying probability space $(\Omega, \mathcal{F}, \mathbb{P})$. The solution becomes a stochastic process, expressed as the formal random series

$$
v(x, t)(\omega)=\sum_{n=1}^{\infty} A_{n}(\omega) \mathrm{e}^{-n^{2} \pi^{2} \beta^{2}(\omega) t} \sin (n \pi x),
$$

where the random Fourier coefficient

$$
A_{n}(\omega)=2 \int_{0}^{1} \psi(y)(\omega) \sin (n \pi y) \mathrm{d} y
$$

is understood as a Lebesgue integral for each $\omega \in \Omega$ (this is sometimes referred to as SP integral, see [129, Def. A-1]). The following result establishes in which sense and under which assumptions the stochastic process $(6.2)-(6.3)$ is a rigorous solution to the randomized PDE problem (6.1) [16, Th. 1.3].

Theorem 6.1 The following statements hold:

i) a.s. (almost surely) solution: Suppose that $\psi \in \mathrm{L}^{2}([0,1] \times \Omega)$. Then the random series that defines (6.2)-(6.3) converges a.s. for all $x \in[0,1]$ and $t>0$. Moreover, $v_{t}(x, t)(\omega)=\beta^{2}(\omega) v_{x x}(x, t)(\omega)$ a.s. for $x \in(0,1)$ and $t>0$, where the derivatives are understood in the classical sense; $v(0, t)(\omega)=v(1, t)(\omega)=0$ a.s. for $t \geq 0$; and $v(x, 0)(\omega)=\psi(x)(\omega)$ a.s. for a.e. (almost everywhere) $x \in[0,1]$.

ii) $\mathrm{L}^{2}$ solution: Suppose that $\psi \in \mathrm{L}^{2}([0,1] \times \Omega)$ and $0<a \leq \beta^{2}(\omega) \leq b$, a.e. $\omega \in \Omega$, for certain $a, b \in \mathbb{R}$. Then the random series that defines (6.2)- 6.3) converges in $\mathrm{L}^{2}(\Omega)$ for all $x \in[0,1]$ and $t>0$. Moreover, 
$v_{t}(x, t)(\omega)=\beta^{2}(\omega) v_{x x}(x, t)(\omega)$ a.s. for $x \in(0,1)$ and $t>0$, where the derivatives are understood in the mean square sense (see Subsection 6.2.2 later); $v(0, t)(\omega)=v(1, t)(\omega)=0$ a.s. for $t \geq 0$; and $v(x, 0)(\omega)=$ $\psi(x)(\omega)$ a.s. for a.e. $x \in[0,1]$.

The main goal of [16] consisted of approximating the probability density function of the stochastic process $v(x, t)(\omega)$ given in $6.2-(6.3)$, for $0<x<1$ and $t>0$. For that purpose, the truncation

$$
v_{N}(x, t)(\omega)=\sum_{n=1}^{N} A_{n}(\omega) \mathrm{e}^{-n^{2} \pi^{2} \beta^{2}(\omega) t} \sin (n \pi x)
$$

was used. By applying the Random Variable Transformation technique, see [89, Lemma 4.12], the density of the truncation $v_{N}(x, t)(\omega)$ was computed and proved the following result [16, Th. 2.8], which provides conditions under which the density function of the solution stochastic process $v(x, t)(\omega)$ from 6.2 - 6.3 can be approximated. Hereinafter, the notation $f_{X}$ stands for the probability density function of the random variable $X$.

Theorem 6.2 Let $\{\psi(x): 0 \leq x \leq 1\}$ be a process in $\mathrm{L}^{2}([0,1] \times \Omega)$. Suppose that $\beta^{2}, A_{1}$ and $\left(A_{2}, \ldots, A_{N}\right)$ are independent and absolutely continuous, for $N \geq 2$. Suppose that the probability density function $f_{A_{1}}$ is Lipschitz on $\mathbb{R}$. Assume that $\sum_{n=m}^{\infty}\left\|\mathrm{e}^{-\left(n^{2}-2\right) \pi^{2} \beta^{2} t}\right\|_{\mathrm{L}^{1}(\Omega)}<\infty$, for certain $m \in \mathbb{N}$. Then the density of $v_{N}(x, t)(\omega)$,

$$
\begin{aligned}
f_{v_{N}(x, t)}(v)= & \int_{\mathbb{R}^{N}} f_{A_{1}}\left(\frac{\mathrm{e}^{\pi^{2} \beta^{2} t}}{\sin (\pi x)}\left\{v-\sum_{n=2}^{N} a_{n} \mathrm{e}^{-n^{2} \pi^{2} \beta^{2} t} \sin (n \pi x)\right\}\right) \\
& \cdot f_{\left(A_{2}, \ldots, A_{N}\right)}\left(a_{2}, \ldots, a_{N}\right) f_{\beta^{2}}\left(\beta^{2}\right) \frac{\mathrm{e}^{\pi^{2} \beta^{2} t}}{\sin (\pi x)} \mathrm{d} a_{2} \cdots \mathrm{d} a_{N} \mathrm{~d} \beta^{2},
\end{aligned}
$$

converges in $\mathrm{L}^{\infty}(\mathbb{R})$ to a density of the random variable $v(x, t)(\omega)$ given in (6.2)- (6.3), for $0<x<1$ and $t>0$.

Moreover, from the proofs in [16, Th. 2.7, Th. 2.8], one has the following rate of convergence for $\left\{f_{v_{N}(x, t)}(v)\right\}_{N=1}^{\infty}$ under the assumptions of Theorem 6.2.

$$
\left|f_{v_{N}(x, t)}(v)-f_{v(x, t)}(v)\right| \leq \frac{2\|\psi\|_{\mathrm{L}^{2}([0,1] \times \Omega)} L}{\sin ^{2}(\pi x)} \sum_{n=N+1}^{\infty}\left\|\mathrm{e}^{-\left(n^{2}-2\right) \pi^{2} \beta^{2} t}\right\|_{\mathrm{L}^{1}(\Omega)},
$$

where $L$ is the Lipschitz constant of $f_{A_{1}}$. 
Another result that could have been added to [16] is presented in what follows. It substitutes the Lipschitz hypothesis by the weaker assumption of a.e. continuity and essential boundedness. The hypothesis $\sum_{n=m}^{\infty}\left\|\mathrm{e}^{-\left(n^{2}-2\right) \pi^{2} \beta^{2} t}\right\|_{\mathrm{L}^{1}(\Omega)}<$ $\infty$ is substituted by $\mathbb{E}\left[\mathrm{e}^{\pi^{2} \beta^{2} t}\right]<\infty$. Then one proves pointwise convergence of the sequence (6.5), so the uniform convergence on $\mathbb{R}$ and the rate of convergence (6.6) are lost.

Remark 6.3 Let $X$ and $Y$ be two independent random variables. If $X$ is absolutely continuous, then $X+Y$ is absolutely continuous. Indeed, for any Borel set $A$, by the convolution formula [8, p. 266] we have $\mathbb{P}(X+Y \in A)=$ $\int_{\mathbb{R}} \mathbb{P}(X \in A-y) \mathbb{P}_{Y}(\mathrm{~d} y)$, where $\mathbb{P}_{Y}=\mathbb{P} \circ Y^{-1}$ is the law of $Y$. If $A$ is null, then $A-y$ is null, so $\mathbb{P}(X \in A-y)=0$. Thus, if $A$ is null, then $\mathbb{P}(X+Y \in A)=0$. By the Radon-Nikodym Theorem [147, Ch. 14], $X+Y$ has a density.

Theorem 6.4 Let $\{\psi(x): 0 \leq x \leq 1\}$ be a process in $\mathrm{L}^{2}([0,1] \times \Omega)$. Suppose that $\beta^{2}, A_{1}$ and $\left(A_{2}, \ldots, A_{N}\right)$ are independent and absolutely continuous, for $N \geq 2$. Suppose that the probability density function $f_{A_{1}}$ is a.e. continuous on $\mathbb{R}$ and $\left\|f_{A_{1}}\right\|_{L^{\infty}(\mathbb{R})}<\infty$. Assume that $\mathbb{E}\left[\mathrm{e}^{\pi^{2} \beta^{2} t}\right]<\infty$. Then the density of $v_{N}(x, t)(\omega)$ given by (6.5),

$$
\begin{aligned}
f_{v_{N}(x, t)}(v)= & \int_{\mathbb{R}^{N}} f_{A_{1}}\left(\frac{\mathrm{e}^{\pi^{2} \beta^{2} t}}{\sin (\pi x)}\left\{v-\sum_{n=2}^{N} a_{n} \mathrm{e}^{-n^{2} \pi^{2} \beta^{2} t} \sin (n \pi x)\right\}\right) \\
& \cdot f_{\left(A_{2}, \ldots, A_{N}\right)}\left(a_{2}, \ldots, a_{N}\right) f_{\beta^{2}}\left(\beta^{2}\right) \frac{\mathrm{e}^{\pi^{2} \beta^{2} t}}{\sin (\pi x)} \mathrm{d} a_{2} \cdots \mathrm{d} a_{N} \mathrm{~d} \beta^{2},
\end{aligned}
$$

converges pointwise to a density of the random variable $v(x, t)(\omega)$ given in (6.2) - 6.3), for all $0<x<1$ and $t>0$.

Proof. Fix $0<x<1, t>0$ and $v \in \mathbb{R}$. From (6.5), notice that

$$
f_{v_{N}(x, t)}(v)=\mathbb{E}\left[f_{A_{1}}\left(\frac{\mathrm{e}^{\pi^{2} \beta^{2} t}}{\sin (\pi x)}\left\{v-\sum_{n=2}^{N} A_{n} \mathrm{e}^{-n^{2} \pi^{2} \beta^{2} t} \sin (n \pi x)\right\}\right) \frac{\mathrm{e}^{\pi^{2} \beta^{2} t}}{\sin (\pi x)}\right] .
$$

Define the random variables

$$
Z_{N}(\omega):=\frac{\mathrm{e}^{\pi^{2} \beta^{2}(\omega) t}}{\sin (\pi x)}\left\{v-\sum_{n=2}^{N} A_{n}(\omega) \mathrm{e}^{-n^{2} \pi^{2} \beta^{2}(\omega) t} \sin (n \pi x)\right\}, Y(\omega):=\frac{\mathrm{e}^{\pi^{2} \beta^{2}(\omega) t}}{\sin (\pi x)} .
$$

By Theorem 6.1 i), we know that

$$
\lim _{N \rightarrow \infty} Z_{N}(\omega)=\frac{\mathrm{e}^{\pi^{2} \beta^{2}(\omega) t}}{\sin (\pi x)}\left\{v-\sum_{n=2}^{\infty} A_{n}(\omega) \mathrm{e}^{-n^{2} \pi^{2} \beta^{2}(\omega) t} \sin (n \pi x)\right\}=: Z(\omega),
$$


for a.e. $\omega \in \Omega$.

By Remark 6.3, $Z$ is absolutely continuous. Thus, since $f_{A_{1}}$ is a.e. continuous, the probability that $Z$ belongs to the discontinuity set of $f_{A_{1}}$ is 0 . By the Continuous Mapping Theorem [135, p. 7, Th. 2.3],

$$
\lim _{N \rightarrow \infty} f_{A_{1}}\left(Z_{N}(\omega)\right) Y(\omega)=f_{A_{1}}(Z(\omega)) Y(\omega),
$$

for a.e. $\omega \in \Omega$. Moreover, $\left|f_{A_{1}}\left(Z_{N}(\omega)\right) Y(\omega)\right| \leq\left\|f_{A_{1}}\right\|_{L^{\infty}(\Omega)}|Y(\omega)|$, being $Y \in \mathrm{L}^{1}(\Omega)$ by the assumption $\mathbb{E}\left[\mathrm{e}^{\pi^{2} \beta^{2} t}\right]<\infty$. By the Dominated Convergence Theorem [113, result 11.32, p. 321],

$$
\lim _{N \rightarrow \infty} f_{v_{N}(x, t)}(v)=\mathbb{E}\left[f_{A_{1}}(Z) Y\right]=: g_{x, t}(v) .
$$

To conclude, we need to show that $g_{x, t}$ is a density of the random variable $v(x, t)(\omega)$ given by $6.2-6.3$. This is done in a similar way to the last part of the proof of Theorem 2.4 in [16]. We know that, for each $0<x<1$ and $t>0, v_{N}(x, t)(\omega) \rightarrow v(x, t)(\omega)$ as $N \rightarrow \infty$ a.s., which implies convergence in law: $F_{v_{N}(x, t)}(v) \rightarrow F_{v(x, t)}(v)$ as $N \rightarrow \infty$, for all $v \in \mathbb{R}$ which is a point of continuity of $F_{v(x, t)}$. Here, $F$ refers to the distribution function. Since $f_{v_{N}(x, t)}$ is the density of $v_{N}(x, t), F_{v_{N}(x, t)}(v)=F_{v_{N}(x, t)}\left(v_{0}\right)+\int_{v_{0}}^{v} f_{v_{N}(x, t)}(w) \mathrm{d} w$. If $v$ and $v_{0}$ are points of continuity of $F_{v(x, t)}$, taking limits when $N \rightarrow \infty$ we get $F_{v(x, t)}(v)=F_{v(x, t)}\left(v_{0}\right)+\int_{v_{0}}^{v} g_{x, t}(w) \mathrm{d} w$. This is justified by the Dominated Convergence Theorem, as

$$
\left|f_{v_{N}(x, t)}(w)\right| \leq \mathbb{E}\left[\left|f_{A_{1}}\left(Z_{N}\right)\right||Y|\right] \leq\left\|f_{A_{1}}\right\|_{\mathrm{L}^{\infty}(\mathbb{R})} \mathbb{E}[Y] \in \mathrm{L}^{1}\left(\left[v_{0}, v\right], \mathrm{d} w\right) .
$$

As the points of discontinuity of $F_{v(x, t)}$ are countable and $F_{v(x, t)}$ is rightcontinuous, we obtain $F_{v(x, t)}(v)=F_{v(x, t)}\left(v_{0}\right)+\int_{v_{0}}^{v} g_{x, t}(w) \mathrm{d} w$, for all $v_{0}$ and $v$ in $\mathbb{R}$. Thus, $g_{x, t}=f_{v(x, t)}$ is a density for $v(x, t)$, as wanted.

Our main objective will be to extend both Theorem 6.2 and the new Theorem 6.4 to the solution of the randomized heat equation on an interval $\left[L_{1}, L_{2}\right]$ with random boundary conditions.

\subsubsection{Preliminaries on the $\mathrm{L}^{1}(\Omega)$ and $\mathrm{L}^{2}(\Omega)$ calculus}

In this section, we summarize the main results related to the so-called $\mathrm{L}^{p}(\Omega)$ random calculus that will be required throughout our subsequent development. To read an exposition on $\mathrm{L}^{2}(\Omega)$ calculus, see [125, Ch. 4] and [89, Ch. 5]. In [137] the authors combined $\mathrm{L}^{2}(\Omega)$ and $\mathrm{L}^{4}(\Omega)$ calculus, usually termed mean 
square and mean fourth random calculus, to solve random differential equations.

With a similar proof to [137, Lemma 3.14], we have the following two results:

Proposition 6.5 Let $X=\{X(t)(\omega): t \in I, \omega \in \Omega\}$ and $Y=\{Y(t)(\omega): t \in$ $I, \omega \in \Omega\}$ be two stochastic processes. Suppose that they are continuous at $t_{0} \in I$ in the $\mathrm{L}^{2}(\Omega)$ sense. Then $X Y=\{X(t)(\omega) Y(t)(\omega): t \in I, \omega \in \Omega\}$ is continuous at $t_{0}$ in the $\mathrm{L}^{1}(\Omega)$ sense.

Proposition 6.6 Let $X=\{X(t)(\omega): t \in I, \omega \in \Omega\}$ and $Y=\{Y(t)(\omega): t \in$ $I, \omega \in \Omega\}$ be two stochastic processes. Suppose that they are differentiable at $t_{0} \in I$ in the $\mathrm{L}^{2}(\Omega)$ sense. Then $X Y=\{X(t)(\omega) Y(t)(\omega): t \in I, \omega \in \Omega\}$ is differentiable at $t_{0}$ in the $\mathrm{L}^{1}(\Omega)$ sense, with $(X Y)^{\prime}\left(t_{0}\right)=X^{\prime}\left(t_{0}\right) Y\left(t_{0}\right)+$ $X\left(t_{0}\right) Y^{\prime}\left(t_{0}\right)$.

Another useful result is the following, see [125, p. 97]:

Proposition 6.7 Let $\left\{X_{n}\right\}_{n=1}^{\infty}$ be a sequence of random variables that converges in $\mathrm{L}^{1}(\Omega)$ to the random variable $X$. Then $\lim _{n \rightarrow \infty} \mathbb{E}\left[X_{n}\right]=\mathbb{E}[X]$. As a consequence, if $X=\{X(t)(\omega): t \in I, \omega \in \omega\}$ is a stochastic process that is differentiable in the $\mathrm{L}^{1}(\Omega)$ sense at $t_{0} \in I$, then there exists $\frac{\mathrm{d}}{\mathrm{d} t} \mathbb{E}\left[X\left(t_{0}\right)\right]=\mathbb{E}\left[X^{\prime}\left(t_{0}\right)\right]$.

A similar result but in terms of continuity holds:

Proposition 6.8 If $X=\{X(t)(\omega): t \in I, \omega \in \omega\}$ is a stochastic process that is continuous in the $\mathrm{L}^{1}(\Omega)$ sense at $t_{0} \in I$, then $\mathbb{E}[X(t)]$ is continuous at $t_{0}$. On the other hand, if $X$ is continuous in the $\mathrm{L}^{2}(\Omega)$ sense at $t_{0} \in I$, then $\|X(t)\|_{\mathrm{L}^{2}(\Omega)}$ is continuous at $t_{0}$.

\subsection{Solution to the randomized heat equation with non-homogeneous Dirichlet boundary conditions}

Consider the general form of the heat equation,

$$
\left\{\begin{array}{l}
u_{t}=\alpha^{2} u_{x x}, \quad L_{1}<x<L_{2}, t>0, \\
u\left(L_{1}, t\right)=A, \quad u\left(L_{2}, t\right)=B, \quad t \geq 0, \\
u(x, 0)=\phi(x), \quad L_{1} \leq x \leq L_{2} .
\end{array}\right.
$$


This is a generalization of the PDE problem (6.1) studied in [16]. Given a complete probability space $(\Omega, \mathcal{F}, \mathbb{P})$, we will consider the diffusion coefficient $\alpha^{2}(\omega)$ and the boundary conditions $A(\omega)$ and $B(\omega)$ as random variables, and the initial condition as a stochastic process $\phi=\left\{\phi(x)(\omega): x \in\left[L_{1}, L_{2}\right], \omega \in\right.$ $\Omega\}$ in the underlying probability space. The solution $u$ can be expressed as

$$
u(x, t)(\omega)=v\left(\frac{x-L_{1}}{L_{2}-L_{1}}, t\right)(\omega)+\frac{x-L_{1}}{L_{2}-L_{1}} B(\omega)+\frac{L_{2}-x}{L_{2}-L_{1}} A(\omega),
$$

where $x \in\left[L_{1}, L_{2}\right]$ and $t \geq 0$, and $v(y, t)(\omega)$ is the solution stochastic process of (6.1) given by $6.2-6.3$ with random diffusion coefficient

$$
\beta^{2}(\omega)=\frac{\alpha^{2}(\omega)}{\left(L_{2}-L_{1}\right)^{2}}
$$

and random initial condition

$$
\begin{aligned}
& \psi(y)(\omega)=\varphi\left(L_{1}+y\left(L_{2}-L_{1}\right)\right)(\omega), \\
& \varphi(x)(\omega)=\phi(x)(\omega)-\frac{x-L_{1}}{L_{2}-L_{1}} B(\omega)-\frac{L_{2}-x}{L_{2}-L_{1}} A(\omega),
\end{aligned}
$$

for $y \in[0,1]$. We want to study in which sense the stochastic process $u(x, t)(\omega)$ given by 6.9 is a rigorous solution to the randomized heat equation 6.8 . Next Theorem 6.9 generalizes Theorem 6.1. Moreover, uniqueness is proved, which is a novelty compared with [16].

Theorem 6.9 The following statements hold:

i) a.s. solution: Suppose that $\phi \in \mathrm{L}^{2}\left(\left[L_{1}, L_{2}\right] \times \Omega\right)$ and $A, B \in \mathrm{L}^{2}(\Omega)$. Then $u_{t}(x, t)(\omega)=\alpha^{2}(\omega) u_{x x}(x, t)(\omega)$ a.s. for $x \in\left(L_{1}, L_{2}\right)$ and $t>0$, where the derivatives are understood in the classical sense; $u\left(L_{1}, t\right)(\omega)=A(\omega)$ and $u\left(L_{2}, t\right)(\omega)=B(\omega)$ a.s. for $t \geq 0$; and $u(x, 0)(\omega)=\phi(x)(\omega)$ a.s. for a.e. $x \in\left[L_{1}, L_{2}\right]$. Moreover, the process $u(x, t)(\omega)$ satisfying these conditions is unique.

ii) $\mathrm{L}^{2}$ solution: Suppose that $\phi \in \mathrm{L}^{2}\left(\left[L_{1}, L_{2}\right] \times \Omega\right), A, B \in \mathrm{L}^{2}(\Omega)$ and $0<a \leq \alpha^{2}(\omega) \leq b$, a.e. $\omega \in \Omega$, for certain $a, b \in \mathbb{R}$. Then $u_{t}(x, t)(\omega)=$ $\alpha^{2}(\omega) u_{x x}(x, t)(\omega)$ a.s. for $x \in\left(L_{1}, L_{2}\right)$ and $t>0$, where the derivatives are understood in the mean square sense; $u\left(L_{1}, t\right)(\omega)=A(\omega)$ and $u\left(L_{2}, t\right)(\omega)=B(\omega)$ a.s. for $t \geq 0$; and $u(x, 0)(\omega)=\phi(x)(\omega)$ a.s. for a.e. $x \in\left[L_{1}, L_{2}\right]$. Moreover, the process $u(x, t)(\omega)$ satisfying these conditions is unique. 
Proof. We prove both statements:

i) a.s. solution: By 6.11 and the triangular inequality,

$$
\begin{aligned}
& \|\psi\|_{\mathrm{L}^{2}([0,1] \times \Omega)}=\left(\mathbb{E}\left[\int_{0}^{1} \psi(y)^{2} \mathrm{~d} y\right]\right)^{\frac{1}{2}}=\left(\mathbb{E}\left[\int_{0}^{1} \varphi\left(L_{1}+y\left(L_{2}-L_{1}\right)\right)^{2} \mathrm{~d} y\right]\right)^{\frac{1}{2}} \\
& =\frac{1}{\sqrt{L_{2}-L_{1}}}\left(\mathbb{E}\left[\int_{L_{1}}^{L_{2}} \varphi(x)^{2} \mathrm{~d} x\right]\right)^{\frac{1}{2}}=\frac{1}{\sqrt{L_{2}-L_{1}}}\|\varphi\|_{\mathrm{L}^{2}\left(\left[L_{1}, L_{2}\right] \times \Omega\right)} \\
& \leq \frac{1}{\sqrt{L_{2}-L_{1}}}\left(\|\phi\|_{\mathrm{L}^{2}\left(\left[L_{1}, L_{2}\right] \times \Omega\right)}+\frac{x-L_{1}}{L_{2}-L_{1}}\|B\|_{\mathrm{L}^{2}\left(\left[L_{1}, L_{2}\right] \times \Omega\right)}+\frac{L_{2}-x}{L_{2}-L_{1}}\|A\|_{\mathrm{L}^{2}\left(\left[L_{1}, L_{2}\right] \times \Omega\right)}\right) \\
& =\frac{1}{\sqrt{L_{2}-L_{1}}}\|\phi\|_{\mathrm{L}^{2}\left(\left[L_{1}, L_{2}\right] \times \Omega\right)}+\frac{x-L_{1}}{L_{2}-L_{1}}\|B\|_{\mathrm{L}^{2}(\Omega)}+\frac{L_{2}-x}{L_{2}-L_{1}}\|A\|_{\mathrm{L}^{2}(\Omega)}<\infty .
\end{aligned}
$$

Then $\psi \in \mathrm{L}^{2}([0,1] \times \Omega)$. By Theorem 6.1, $v_{t}(x, t)(\omega)=\beta^{2}(\omega) v_{x x}(x, t)(\omega)$ a.s. for $x \in(0,1)$ and $t>0$, where the derivatives are understood in the classical sense; $v(0, t)(\omega)=v(1, t)(\omega)=0$ a.s. for $t \geq 0$; and $v(x, 0)(\omega)=\psi(x)(\omega)$ a.s. for a.e. $x \in[0,1]$. Then $u$ is an almost sure solution. Uniqueness follows from the so-called energy method [115, pp. 30-31] applied to each sample path.

ii) $\mathrm{L}^{2}$ solution: the result follows as a consequence of Theorem 6.1. To show uniqueness, we try to adapt the energy method [115, pp. 30-31] to this setting. We prove the following: if $u$ is $C^{2,1}\left(\left(L_{1}, L_{2}\right) \times(0, \infty)\right)$ in the sense of $\mathrm{L}^{2}(\Omega)$, with continuous partial derivatives on $\left[L_{1}, L_{2}\right] \times[0, \infty)$ in the sense of $\mathrm{L}^{2}(\Omega)$, $u_{t}=\alpha^{2} u_{x x}$ on $\left(L_{1}, L_{2}\right) \times(0, \infty), u\left(L_{1}, t\right)=u\left(L_{2}, t\right)=0$ a.s. on $[0, \infty)$ and $u(x, 0)=0$ a.s. at a.e. $x \in\left[L_{1}, L_{2}\right]$, then $u(x, t)=0$ a.s. for all $x \in\left[L_{1}, L_{2}\right]$ and $t \geq 0$. From this fact, uniqueness will follow.

Let $I(t)=\int_{L_{1}}^{L_{2}} \mathbb{E}\left[u(x, t)^{2}\right] \mathrm{d} x$. Fixed $t \geq 0$, as a consequence of the continuity of $u(\cdot, t)$ in the $\mathrm{L}^{2}(\Omega)$ sense and Proposition 6.8, the real map $x \in$ $\left[L_{1}, L_{2}\right] \mapsto \mathbb{E}\left[u(x, t)^{2}\right]$ is continuous and $I(t)$ is well-defined. Fixed $x$, as $u(x, \cdot)$ is differentiable in the $\mathrm{L}^{2}(\Omega)$ sense, by Proposition 6.6 and Proposition 6.7 we have $\frac{\partial}{\partial t} \mathbb{E}\left[u(x, t)^{2}\right]=\mathbb{E}\left[\frac{\partial}{\partial t}\left(u(x, t)^{2}\right)\right]=2 \mathbb{E}\left[u(x, t) u_{t}(x, t)\right]$, where the partial derivative $\frac{\partial}{\partial t}$ inside the expectation operator must be understood on the $\mathrm{L}^{1}(\Omega)$ sense. Then, using Cauchy-Schwarz inequality, $\left|\frac{\partial}{\partial t} \mathbb{E}\left[u(x, t)^{2}\right]\right| \leq$ $2 \mathbb{E}\left[\left|u(x, t) \| u_{t}(x, t)\right|\right] \leq 2\|u(x, t)\|_{\mathrm{L}^{2}(\Omega)}\left\|u_{t}(x, t)\right\|_{\mathrm{L}^{2}(\Omega)}$. As both $u(x, t), u_{t}(x, t)$ are continuous on $\left[L_{1}, L_{2}\right] \times[0, \infty)$ in the $\mathrm{L}^{2}(\Omega)$ sense, by Proposition 6.8 both $\|u(x, t)\|_{\mathrm{L}^{2}(\Omega)}$ and $\left\|u_{t}(x, t)\right\|_{\mathrm{L}^{2}(\Omega)}$ are continuous on $\left[L_{1}, L_{2}\right] \times[0, \infty)$ in the classical sense. Fix $t_{0}>0$ and $\delta>0$ small. Then there is a constant $C>0$ such that $\|u(x, t)\|_{\mathrm{L}^{2}(\Omega)} \leq C$ and $\left\|u_{t}(x, t)\right\|_{\mathrm{L}^{2}(\Omega)} \leq C$ for all $x \in\left[L_{1}, L_{2}\right]$ and $t \in\left[t_{0}-\delta, t_{0}+\delta\right]$, by continuity in the classical sense. Thus, $\left|\frac{\partial}{\partial t} \mathbb{E}\left[u(x, t)^{2}\right]\right| \leq$ $2 C^{2} \in \mathrm{L}^{1}\left(\left[L_{1}, L_{2}\right], \mathrm{d} x\right)$, for all $x \in\left[L_{1}, L_{2}\right]$ and $t \in\left[t_{0}-\delta, t_{0}+\delta\right]$. This permits 
differentiating under the Lebesgue integral sign at $t_{0}$ [2, Th. 10.39]:

$$
\begin{aligned}
I^{\prime}\left(t_{0}\right) & =\left.\frac{\partial}{\partial t}\left(\int_{L_{1}}^{L_{2}} \mathbb{E}\left[u(x, t)^{2}\right] \mathrm{d} x\right)\right|_{t=t_{0}}=\left.\int_{L_{1}}^{L_{2}} \frac{\partial}{\partial t}\left(\mathbb{E}\left[u(x, t)^{2}\right]\right)\right|_{t=t_{0}} \mathrm{~d} x \\
& =2 \int_{L_{1}}^{L_{2}} \mathbb{E}\left[u\left(x, t_{0}\right) u_{t}\left(x, t_{0}\right)\right] \mathrm{d} x .
\end{aligned}
$$

Now we use the arbitrariness of $t_{0}$ and the fact that $u$ solves the heat equation:

$$
I^{\prime}(t)=2 \int_{L_{1}}^{L_{2}} \mathbb{E}\left[u(x, t) u_{t}(x, t)\right] \mathrm{d} x=2 \int_{L_{1}}^{L_{2}} \mathbb{E}\left[\alpha^{2} u(x, t) u_{x x}(x, t)\right] \mathrm{d} x .
$$

As $u(\cdot, t)$ and $u_{x}(\cdot, t)$ are differentiable in the $\mathrm{L}^{2}(\Omega)$ sense, by Proposition 6.6 the product $u(\cdot, t) u_{x}(\cdot, t)$ is differentiable in $\mathrm{L}^{1}(\Omega),\left(u(x, t) u_{x}(x, t)\right)_{x}=$ $u(x, t) u_{x x}(x, t)+u_{x}(x, t)^{2}$. Since $\alpha^{2}$ is bounded above, $\alpha^{2} u(\cdot, t) u_{x}(\cdot, t)$ is differentiable in the $\mathrm{L}^{1}(\Omega)$ sense, having derivative

$$
\left(\alpha^{2} u(x, t) u_{x}(x, t)\right)_{x}=\alpha^{2} u(x, t) u_{x x}(x, t)+\alpha^{2} u_{x}(x, t)^{2} .
$$

Thereby,

$$
I^{\prime}(t)=2 \int_{L_{1}}^{L_{2}} \mathbb{E}\left[\left(\alpha^{2} u(x, t) u_{x}(x, t)\right)_{x}\right] \mathrm{d} x-2 \int_{L_{1}}^{L_{2}} \mathbb{E}\left[\alpha^{2} u_{x}(x, t)^{2}\right] \mathrm{d} x .
$$

By Proposition 6.7, Barrow's rule and the boundary conditions, the first integral is 0 :

$$
\begin{gathered}
\int_{L_{1}}^{L_{2}} \mathbb{E}\left[\left(\alpha^{2} u(x, t) u_{x}(x, t)\right)_{x}\right] \mathrm{d} x=\int_{L_{1}}^{L_{2}} \frac{\partial}{\partial x} \mathbb{E}\left[\alpha^{2} u(x, t) u_{x}(x, t)\right] \mathrm{d} x \\
=\mathbb{E}\left[\alpha^{2} u\left(L_{2}, t\right) u_{x}\left(L_{2}, t\right)\right]-\mathbb{E}\left[\alpha^{2} u\left(L_{1}, t\right) u_{x}\left(L_{1}, t\right)\right]=0 .
\end{gathered}
$$

Barrow's rule is justified as follows: we have, by previous computations,

$$
\begin{aligned}
\partial_{x} \mathbb{E}\left[\alpha^{2} u(x, t) u_{x}(x, t)\right] & =\mathbb{E}\left[\left(\alpha^{2} u(x, t) u_{x}(x, t)\right)_{x}\right] \\
& =\mathbb{E}\left[\alpha^{2} u(x, t) u_{x x}(x, t)\right]+\mathbb{E}\left[\alpha^{2} u_{x}(x, t)^{2}\right] .
\end{aligned}
$$

By Proposition 6.5 and the boundedness of $\alpha^{2}$, both $\alpha^{2} u(\cdot, t) u_{x x}(\cdot, t)$ and $\alpha^{2} u_{x}(\cdot, t)^{2}$ are continuous in the $\mathrm{L}^{1}(\Omega)$ sense. So by Proposition 6.8, both $\mathbb{E}\left[\alpha^{2} u(\cdot, t) u_{x x}(\cdot, t)\right]$ and $\mathbb{E}\left[\alpha^{2} u_{x}(\cdot, t)^{2}\right]$ are continuous. Then $\partial_{x} \mathbb{E}\left[\alpha^{2} u(\cdot, t) u_{x}(\cdot, t)\right]$ is continuous on $\left[L_{1}, L_{2}\right]$ and Barrow's rule is applicable.

It follows $I^{\prime}(t)=-2 \int_{L_{1}}^{L_{2}} \mathbb{E}\left[\alpha^{2} u_{x}(x, t)^{2}\right] \mathrm{d} x \leq 0$. This tells us that $I(t)$ is decreasing on $[0, \infty)$, which implies $I(t) \leq I(0)=\int_{L_{1}}^{L_{2}} \mathbb{E}\left[u(x, 0)^{2}\right] \mathrm{d} x=0$. Hence, $I(t)=0$. As $\mathbb{E}\left[u(\cdot, t)^{2}\right]$ is continuous, because $u(\cdot, t)$ is continuous in the $\mathrm{L}^{2}(\Omega)$ 
sense and Proposition 6.8, we derive that $\mathbb{E}\left[u(x, t)^{2}\right]=0$ for all $x \in\left[L_{1}, L_{2}\right]$ and $t \geq 0$. Then $u(x, t)=0$ a.s., for every $x \in\left[L_{1}, L_{2}\right]$ and $t \geq 0$. This concludes the proof.

\subsection{Approximation of the probability density function of the solution stochastic process}

The main goal of this chapter is to approximate the probability density function of the solution stochastic process $u(x, t)(\omega)$ given by $(6.9)$, which solves the random heat equation $(6.8)$. We will use Theorem 6.2, Theorem 6.4 and the Random Variable Transformation technique [89, Lemma 4.12].

Assume that $v(y, t)(\omega), A(\omega)$ and $B(\omega)$ are absolutely continuous and independent random variables. Applying the Random Variable Transformation technique,

$f_{\frac{x-L_{1}}{L_{2}-L_{1}} B}(b)=f_{B}\left(\frac{L_{2}-L_{1}}{x-L_{1}} b\right) \frac{L_{2}-L_{1}}{x-L_{1}}, f_{\frac{L_{2}-x}{L_{2}-L_{1}} A}(a)=f_{A}\left(\frac{L_{2}-L_{1}}{L_{2}-x} a\right) \frac{L_{2}-L_{1}}{L_{2}-x}$.

It is well-known, see [8, p. 267], [107, p. 372], that the probability density function of a sum of two independent and absolutely continuous random variables is given by the convolution of their probability density functions. Thereby, from $(6.13)$,

$$
\begin{aligned}
& f_{u(x, t)}(u)=\int_{\mathbb{R}} \int_{\mathbb{R}} f_{v\left(\frac{x-L_{1}}{L_{2}-L_{1}}, t\right)}(u-b-a) f_{\frac{x-L_{1}}{L_{2}-L_{1}} B}(b) f_{\frac{L_{2}-x}{L_{2}-L_{1}} A}(a) \mathrm{d} a \mathrm{~d} b \\
= & \int_{\mathbb{R}^{2}} f_{v\left(\frac{x-L_{1}}{L_{2}-L_{1}}, t\right)}(u-b-a) f_{B}\left(\frac{L_{2}-L_{1}}{x-L_{1}} b\right) \frac{L_{2}-L_{1}}{x-L_{1}} f_{A}\left(\frac{L_{2}-L_{1}}{L_{2}-x} a\right) \frac{L_{2}-L_{1}}{L_{2}-x} \mathrm{~d} a \mathrm{~d} b .
\end{aligned}
$$

Define a new truncation from 6.9

$$
u_{N}(x, t)(\omega)=v_{N}\left(\frac{x-L_{1}}{L_{2}-L_{1}}, t\right)(\omega)+\frac{x-L_{1}}{L_{2}-L_{1}} B(\omega)+\frac{L_{2}-x}{L_{2}-L_{1}} A(\omega),
$$

where $x \in\left[L_{1}, L_{2}\right]$ and $t \geq 0$ and $v_{N}$ is the truncation (6.4). If $v_{N}(y, t)(\omega)$, $A(\omega)$ and $B(\omega)$ are absolutely continuous and independent random variables,

$$
\begin{aligned}
& f_{u_{N}(x, t)}(u)=\int_{\mathbb{R}} \int_{\mathbb{R}} f_{v_{N}\left(\frac{x-L_{1}}{L_{2}-L_{1}}, t\right)}(u-b-a) f_{\frac{x-L_{1}}{L_{2}-L_{1}} B}(b) f_{\frac{L_{2}-x}{L_{2}-L_{1}} A}(a) \mathrm{d} a \mathrm{~d} b \\
= & \int_{\mathbb{R}^{2}} f_{v_{N}\left(\frac{x-L_{1}}{L_{2}-L_{1}}, t\right)}(u-b-a) f_{B}\left(\frac{L_{2}-L_{1}}{x-L_{1}} b\right) \frac{L_{2}-L_{1}}{x-L_{1}} f_{A}\left(\frac{L_{2}-L_{1}}{L_{2}-x} a\right) \frac{L_{2}-L_{1}}{L_{2}-x} \mathrm{~d} a \mathrm{~d} b .
\end{aligned}
$$


Intuitively, we should be able to set conditions under which the convergence $\lim _{N \rightarrow \infty} f_{u_{N}(x, t)}(u)=f_{u(x, t)}(u)$ holds, as an application of Theorem 6.2 or of Theorem 6.4. This fact is formalized in the following two theorems.

Theorem 6.10 Let the random initial condition $\left\{\phi(x): L_{1} \leq x \leq L_{2}\right\}$ be a process in $\mathrm{L}^{2}\left(\left[L_{1}, L_{2}\right] \times \Omega\right)$. Let the random boundary conditions $A$ and $B$ belong to $\mathrm{L}^{2}(\Omega)$. Suppose that $\alpha^{2}, A_{1},\left(A_{2}, \ldots, A_{N}\right), A$ and $B$ are independent and absolutely continuous, for $N \geq 2$ (recall that $A_{n}$ is defined in (6.3) as the random Fourier coefficient of $\psi$, where $\psi$ is defined from $\phi$ in relation (6.11)). Suppose that the probability density function $f_{A_{1}}$ is Lipschitz on $\mathbb{R}$. Assume that $\sum_{n=m}^{\infty}\left\|\mathrm{e}^{-\left(n^{2}-2\right) \pi^{2} \alpha^{2} t /\left(L_{2}-L_{1}\right)^{2}}\right\|_{\mathrm{L}^{1}(\Omega)}<\infty$, for certain $m \in \mathbb{N}$. Then the sequence

$$
\begin{aligned}
f_{u_{N}(x, t)}(u)= & \int_{\mathbb{R}} \int_{\mathbb{R}} f_{v_{N}\left(\frac{x-L_{1}}{L_{2}-L_{1}}, t\right)}(u-b-a) f_{B}\left(\frac{L_{2}-L_{1}}{x-L_{1}} b\right) \frac{L_{2}-L_{1}}{x-L_{1}} \\
& \cdot f_{A}\left(\frac{L_{2}-L_{1}}{L_{2}-x} a\right) \frac{L_{2}-L_{1}}{L_{2}-x} \mathrm{~d} a \mathrm{~d} b,
\end{aligned}
$$

where $f_{v_{N}}$ is the density defined by 6.5$)$, converges in $\mathrm{L}^{\infty}(\mathbb{R})$ to the density $f_{u(x, t)}(u)$ of the solution stochastic process $u(x, t)(\omega)$ to the randomized heat equation (6.8), for $L_{1}<x<L_{2}$ and $t>0$.

Proof. Since $\phi \in \mathrm{L}^{2}\left(\left[L_{1}, L_{2}\right] \times \Omega\right)$ and by 6.12$), \psi \in \mathrm{L}^{2}([0,1] \times \Omega)$. By hypothesis, we also have that $\beta^{2}=\alpha^{2} /\left(L_{2}-L_{1}\right)^{2}, A_{1}$ and $\left(A_{2}, \ldots, A_{N}\right)$ are independent and absolutely continuous, for $N \geq 2$, and $\sum_{n=m}^{\infty}\left\|\mathrm{e}^{-\left(n^{2}-2\right) \pi^{2} \beta^{2} t}\right\|_{\mathrm{L}^{1}(\Omega)}<$ $\infty$. Thus, the hypotheses of Theorem 6.2 hold.

Since $\alpha^{2}, A_{1},\left(A_{2}, \ldots, A_{N}\right), A$ and $B$ are independent, from $(6.2)$ we derive that $v(y, t), A$ and $B$ are independent. Indeed, from the independence of $\beta^{2}=\alpha^{2} /\left(L_{2}-L_{1}\right)^{2}, A_{1},\left(A_{2}, \ldots, A_{N}\right), A$ and $B$, one has independence of $\left(\beta^{2}, A_{1}, \ldots, A_{N}\right), A$ and $B$. Fixed $0<y<1$ and $t>0$, the random variable $v_{N}(y, t)(\omega)$ can be written as $g\left(\beta^{2}(\omega), A_{1}(\omega), \ldots, A_{N}(\omega)\right)$, for a Borel measurable map $g: \mathbb{R}^{n+1} \rightarrow \mathbb{R}$. Then $v_{N}(y, t), A$ and $B$ are independent. By Theorem 6.1 i), $v_{N}(y, t) \rightarrow v(y, t)$ a.s. as $N \rightarrow \infty$. Then $\left(v_{N}(y, t), A, B\right) \rightarrow$ $(v(y, t), A, B)$ a.s. as $N \rightarrow \infty$. Denote by $\hat{\varphi}$ the characteristic function. By Lévy's Continuity Theorem [141, Ch. 18] and the independence, for $v, a, b \in \mathbb{R}$, we have these equalities: $\hat{\varphi}_{(v(y, t), A, B)}(v, a, b)=\lim _{N \rightarrow \infty} \hat{\varphi}_{\left(v_{N}(y, t), A, B\right)}(v, a, b)=$ $\lim _{N \rightarrow \infty} \hat{\varphi}_{v_{N}(y, t)}(v) \hat{\varphi}_{A}(a) \hat{\varphi}_{B}(b)=\hat{\varphi}_{v(y, t)}(v) \hat{\varphi}_{A}(a) \hat{\varphi}_{B}(b)$. By [3, Th. 2.1], $v(y, t)$, 
$A$ and $B$ are independent. As a consequence,

$$
\begin{aligned}
f_{u_{N}(x, t)}(u)= & \int_{\mathbb{R}} \int_{\mathbb{R}} f_{v_{N}\left(\frac{x-L_{1}}{L_{2}-L_{1}}, t\right)}(u-b-a) f_{B}\left(\frac{L_{2}-L_{1}}{x-L_{1}} b\right) \frac{L_{2}-L_{1}}{x-L_{1}} \\
& \cdot f_{A}\left(\frac{L_{2}-L_{1}}{L_{2}-x} a\right) \frac{L_{2}-L_{1}}{L_{2}-x} \mathrm{~d} a \mathrm{~d} b
\end{aligned}
$$

and

$$
\begin{aligned}
f_{u(x, t)}(u)= & \int_{\mathbb{R}} \int_{\mathbb{R}} f_{v\left(\frac{x-L_{1}}{L_{2}-L_{1}}, t\right)}(u-b-a) f_{B}\left(\frac{L_{2}-L_{1}}{x-L_{1}} b\right) \frac{L_{2}-L_{1}}{x-L_{1}} \\
& \cdot f_{A}\left(\frac{L_{2}-L_{1}}{L_{2}-x} a\right) \frac{L_{2}-L_{1}}{L_{2}-x} \mathrm{~d} a \mathrm{~d} b .
\end{aligned}
$$

We have the following estimates:

$$
\begin{aligned}
& \left|f_{u(x, t)}(u)-f_{u_{N}(x, t)}(u)\right| \\
= & \mid \int_{\mathbb{R}} \int_{\mathbb{R}} f_{v\left(\frac{x-L_{1}}{L_{2}-L_{1}}, t\right)}(u-b-a) f_{B}\left(\frac{L_{2}-L_{1}}{x-L_{1}} b\right) \frac{L_{2}-L_{1}}{x-L_{1}} f_{A}\left(\frac{L_{2}-L_{1}}{L_{2}-x} a\right) \frac{L_{2}-L_{1}}{L_{2}-x} \mathrm{~d} a \mathrm{~d} b \\
& -\int_{\mathbb{R}} \int_{\mathbb{R}} f_{v_{N}\left(\frac{x-L_{1}}{L_{2}-L_{1}}, t\right)}(u-b-a) f_{B}\left(\frac{L_{2}-L_{1}}{x-L_{1}} b\right) \frac{L_{2}-L_{1}}{x-L_{1}} f_{A}\left(\frac{L_{2}-L_{1}}{L_{2}-x} a\right) \frac{L_{2}-L_{1}}{L_{2}-x} \mathrm{~d} a \mathrm{~d} b \mid \\
\leq & \frac{\left(L_{2}-L_{1}\right)^{2}}{\left(x-L_{1}\right)\left(L_{2}-x\right)} \int_{\mathbb{R}} \int_{\mathbb{R}} f_{B}\left(\frac{L_{2}-L_{1}}{x-L_{1}} b\right) f_{A}\left(\frac{L_{2}-L_{1}}{L_{2}-x} a\right) \\
& \cdot\left|f_{v\left(\frac{x-L_{1}}{L_{2}-L_{1}}, t\right)}(u-b-a)-f_{v_{N}\left(\frac{x-L_{1}}{L_{2}-L_{1}}, t\right)}(u-b-a)\right| \mathrm{d} a \mathrm{~d} b .
\end{aligned}
$$

By 6.6,

$$
\begin{aligned}
& \left|f_{v\left(\frac{x-L_{1}}{L_{2}-L_{1}}, t\right)}(u-b-a)-f_{v_{N}\left(\frac{x-L_{1}}{L_{2}-L_{1}}, t\right)}(u-b-a)\right| \\
\leq & \frac{2\|\psi\|_{L^{2}([0,1] \times \Omega)} L}{\sin ^{2}\left(\pi \frac{x-L_{1}}{L_{2}-L_{1}}\right)} \sum_{n=N+1}^{\infty}\left\|\mathrm{e}^{-\left(n^{2}-2\right) \pi^{2} \alpha^{2} t /\left(L_{2}-L_{1}\right)^{2}}\right\|_{\mathrm{L}^{1}(\Omega)},
\end{aligned}
$$


where $L$ is the Lipschitz constant of $f_{A_{1}}$. Then,

$$
\begin{aligned}
& \left|f_{u_{N}(x, t)}(u)-f_{u(x, t)}(u)\right| \\
\leq & \frac{\left(L_{2}-L_{1}\right)^{2}}{\left(x-L_{1}\right)\left(L_{2}-x\right)} \frac{2\|\psi\|_{\mathrm{L}^{2}([0,1] \times \Omega)} L}{\sin ^{2}\left(\pi \frac{x-L_{1}}{L_{2}-L_{1}}\right)}\left(\sum_{n=N+1}^{\infty}\left\|\mathrm{e}^{-\left(n^{2}-2\right) \pi^{2} \alpha^{2} t /\left(L_{2}-L_{1}\right)^{2}}\right\|_{\mathrm{L}^{1}(\Omega)}\right) \\
& \cdot \int_{\mathbb{R}} \int_{\mathbb{R}} f_{B}\left(\frac{L_{2}-L_{1}}{x-L_{1}} b\right) f_{A}\left(\frac{L_{2}-L_{1}}{L_{2}-x} a\right) \mathrm{d} a \mathrm{~d} b \\
= & \frac{\left(L_{2}-L_{1}\right)^{2}}{\left(x-L_{1}\right)\left(L_{2}-x\right)} \frac{2\|\psi\|_{\mathrm{L}^{2}([0,1] \times \Omega)} L}{\sin ^{2}\left(\pi \frac{x-L_{1}}{L_{2}-L_{1}}\right)}\left(\sum_{n=N+1}^{\infty}\left\|\mathrm{e}^{-\left(n^{2}-2\right) \pi^{2} \alpha^{2} t /\left(L_{2}-L_{1}\right)^{2}}\right\|_{\mathrm{L}^{1}(\Omega)}\right) \\
& \cdot\left(\int_{\mathbb{R}} f_{B}\left(\frac{L_{2}-L_{1}}{x-L_{1}} b\right) \mathrm{d} b\right)\left(\int_{\mathbb{R}} f_{A}\left(\frac{L_{2}-L_{1}}{L_{2}-x} a\right) \mathrm{d} a\right) \\
= & \left\|f_{A}\right\|_{\mathrm{L}^{1}(\mathbb{R})}\left\|f_{B}\right\|_{\mathrm{L}^{1}(\mathbb{R})} \frac{2\|\psi\|_{\mathrm{L}^{2}([0,1] \times \Omega)} L}{\sin ^{2}\left(\pi \frac{x-L_{1}}{L_{2}-L_{1}}\right)} \sum_{n=N+1}^{\infty}\left\|\mathrm{e}^{-\left(n^{2}-2\right) \pi^{2} \alpha^{2} t /\left(L_{2}-L_{1}\right)^{2}}\right\|_{\mathrm{L}^{1}(\Omega)} .
\end{aligned}
$$

As $\sum_{n=m}^{\infty}\left\|\mathrm{e}^{-\left(n^{2}-2\right) \pi^{2} \alpha^{2} t /\left(L_{2}-L_{1}\right)^{2}}\right\|_{\mathrm{L}^{1}(\Omega)}<\infty$, we conclude that

$$
\lim _{N \rightarrow \infty} f_{u_{N}(x, t)}(u)=f_{u(x, t)}(u)
$$

in $\mathrm{L}^{\infty}(\mathbb{R})$, with convergence rate given by 6.16 .

Theorem 6.11 Let the random initial condition $\left\{\phi(x): L_{1} \leq x \leq L_{2}\right\}$ be a process in $\mathrm{L}^{2}\left(\left[L_{1}, L_{2}\right] \times \Omega\right)$. Let the random boundary conditions $A$ and $B$ belong to $\mathrm{L}^{2}(\Omega)$. Suppose that $\alpha^{2}, A_{1},\left(A_{2}, \ldots, A_{N}\right), A$ and $B$ are independent and absolutely continuous, for $N \geq 2$ (recall that $A_{n}$ is defined in (6.3) as the random Fourier coefficient of $\psi$, where $\psi$ is defined from $\phi$ in relation (6.11)). Suppose that the probability density function $f_{A_{1}}$ is a.e. continuous on $\mathbb{R}$ and $\left\|f_{A_{1}}\right\|_{\mathrm{L}^{\infty}(\mathbb{R})}<\infty$. Assume that $\mathbb{E}\left[\mathrm{e}^{\pi^{2} \alpha^{2} t /\left(L_{2}-L_{1}\right)^{2}}\right]<\infty$. Then the sequence

$$
\begin{aligned}
f_{u_{N}(x, t)}(u)= & \int_{\mathbb{R}} \int_{\mathbb{R}} f_{v_{N}\left(\frac{x-L_{1}}{L_{2}-L_{1}}, t\right)}(u-b-a) f_{B}\left(\frac{L_{2}-L_{1}}{x-L_{1}} b\right) \frac{L_{2}-L_{1}}{x-L_{1}} \\
& \cdot f_{A}\left(\frac{L_{2}-L_{1}}{L_{2}-x} a\right) \frac{L_{2}-L_{1}}{L_{2}-x} \mathrm{~d} a \mathrm{~d} b,
\end{aligned}
$$

where $f_{v_{N}}$ is the density defined by (6.5), converges pointwise to the density $f_{u(x, t)}(u)$ of the solution stochastic process $u(x, t)(\omega)$ to the randomized heat equation (6.8), for $L_{1}<x<L_{2}$ and $t>0$. 
Proof. From $\phi \in \mathrm{L}^{2}\left(\left[L_{1}, L_{2}\right] \times \Omega\right)$ and $(6.12)$, it follows $\psi \in \mathrm{L}^{2}([0,1] \times \Omega)$. By hypothesis, we also have that $\beta^{2}=\alpha^{2} /\left(L_{2}-L_{1}\right)^{2}, A_{1}$ and $\left(A_{2}, \ldots, A_{N}\right)$ are independent and absolutely continuous, for $N \geq 2$, and $\mathbb{E}\left[\mathrm{e}^{\pi^{2} \beta^{2} t}\right]<\infty$. Thereby, the hypotheses of Theorem 6.4 are fulfilled.

Since $\alpha^{2}, A_{1},\left(A_{2}, \ldots, A_{N}\right), A$ and $B$ are independent, as we did in the proof of Theorem 6.10 we deduce that $v_{N}(y, t), A$ and $B$ are independent, and that $v(y, t), A$ and $B$ are independent. Hence,

$$
\begin{aligned}
f_{u_{N}(x, t)}(u)= & \int_{\mathbb{R}} \int_{\mathbb{R}} f_{v_{N}\left(\frac{x-L_{1}}{L_{2}-L_{1}}, t\right)}(u-b-a) f_{B}\left(\frac{L_{2}-L_{1}}{x-L_{1}} b\right) \frac{L_{2}-L_{1}}{x-L_{1}} \\
& \cdot f_{A}\left(\frac{L_{2}-L_{1}}{L_{2}-x} a\right) \frac{L_{2}-L_{1}}{L_{2}-x} \mathrm{~d} a \mathrm{~d} b
\end{aligned}
$$

and

$$
\begin{aligned}
f_{u(x, t)}(u)= & \int_{\mathbb{R}} \int_{\mathbb{R}} f_{v\left(\frac{x-L_{1}}{L_{2}-L_{1}}, t\right)}(u-b-a) f_{B}\left(\frac{L_{2}-L_{1}}{x-L_{1}} b\right) \frac{L_{2}-L_{1}}{x-L_{1}} \\
& \cdot f_{A}\left(\frac{L_{2}-L_{1}}{L_{2}-x} a\right) \frac{L_{2}-L_{1}}{L_{2}-x} \mathrm{~d} a \mathrm{~d} b .
\end{aligned}
$$

By Theorem 6.4,

$$
\begin{gathered}
\lim _{N \rightarrow \infty} f_{v_{N}\left(\frac{x-L_{1}}{L_{2}-L_{1}}, t\right)}(u-b-a) f_{B}\left(\frac{L_{2}-L_{1}}{x-L_{1}} b\right) \frac{L_{2}-L_{1}}{x-L_{1}} f_{A}\left(\frac{L_{2}-L_{1}}{L_{2}-x} a\right) \frac{L_{2}-L_{1}}{L_{2}-x} \\
\quad=f_{v\left(\frac{x-L_{1}}{L_{2}-L_{1}}, t\right)}(u-b-a) f_{B}\left(\frac{L_{2}-L_{1}}{x-L_{1}} b\right) \frac{L_{2}-L_{1}}{x-L_{1}} f_{A}\left(\frac{L_{2}-L_{1}}{L_{2}-x} a\right) \frac{L_{2}-L_{1}}{L_{2}-x}
\end{gathered}
$$

for every $u, a, b \in \mathbb{R}, L_{1}<x<L_{2}$ and $t>0$. By 6.7),

$$
\begin{aligned}
& \left|f_{v_{N}\left(\frac{x-L_{1}}{L_{2}-L_{1}}, t\right)}(u-b-a) f_{B}\left(\frac{L_{2}-L_{1}}{x-L_{1}} b\right) \frac{L_{2}-L_{1}}{x-L_{1}} f_{A}\left(\frac{L_{2}-L_{1}}{L_{2}-x} a\right) \frac{L_{2}-L_{1}}{L_{2}-x}\right| \\
\leq & \left\|f_{A_{1}}\right\|_{\mathrm{L}^{\infty}(\mathbb{R})} \frac{\mathbb{E}\left[\mathrm{e}^{\pi^{2} \beta^{2} t}\right]}{\sin \left(\pi \frac{x-L_{1}}{L_{2}-L_{1}}\right)} \frac{\left(L_{2}-L_{1}\right)^{2}}{\left(x-L_{1}\right)\left(L_{2}-x\right)} f_{B}\left(\frac{L_{2}-L_{1}}{x-L_{1}} b\right) f_{A}\left(\frac{L_{2}-L_{1}}{L_{2}-x} a\right) \\
\in & \mathrm{L}^{1}\left(\mathbb{R}^{2}, \mathrm{~d} a \mathrm{~d} b\right),
\end{aligned}
$$

so by the Dominated Convergence Theorem, $\lim _{N \rightarrow \infty} f_{u_{N}(x, t)}(u)=f_{u(x, t)}(u)$ follows.

Theorem 6.10 and Theorem 6.11 may be adapted to the case in which $A$ and $B$ are deterministic. By applying the Random Variable Transformation 
technique in 6.9 and 6.14 ,

$$
f_{u(x, t)}(u)=f_{v\left(\frac{x-L_{1}}{L_{2}-L_{1}}, t\right)}\left(u-\frac{x-L_{1}}{L_{2}-L_{1}} B-\frac{L_{2}-x}{L_{2}-L_{1}} A\right)
$$

and

$$
f_{u_{N}(x, t)}(u)=f_{v_{N}\left(\frac{x-L_{1}}{L_{2}-L_{1}}, t\right)}\left(u-\frac{x-L_{1}}{L_{2}-L_{1}} B-\frac{L_{2}-x}{L_{2}-L_{1}} A\right) .
$$

One arrives at the following two theorems, which are proved similarly but easier than Theorem 6.10 and Theorem 6.11, respectively.

Theorem 6.12 Let the random initial condition $\left\{\phi(x): L_{1} \leq x \leq L_{2}\right\}$ be a process in $\mathrm{L}^{2}\left(\left[L_{1}, L_{2}\right] \times \Omega\right)$. Suppose that $\alpha^{2}, A_{1}$ and $\left(A_{2}, \ldots, A_{N}\right)$ are independent and absolutely continuous, for $N \geq 2$ (recall that $A_{n}$ is defined in (6.3) as the random Fourier coefficient of $\psi$, where $\psi$ is defined from $\phi$ in relation (6.11)). Suppose that the probability density function $f_{A_{1}}$ is Lipschitz on $\mathbb{R}$ and that $\sum_{n=m}^{\infty}\left\|\mathrm{e}^{-\left(n^{2}-2\right) \pi^{2} \alpha^{2} t /\left(L_{2}-L_{1}\right)^{2}}\right\|_{\mathrm{L}^{1}(\Omega)}<\infty$, for certain $m \in \mathbb{N}$. Then the sequence defined by (6.17) where $f_{v_{N}}$ is the density defined by (6.5), converges in $\mathrm{L}^{\infty}(\mathbb{R})$ to the density $f_{u(x, t)}(u)$ of the solution stochastic process $u(x, t)(\omega)$ to the randomized heat equation (6.8) with deterministic boundary conditions $A$ and $B$, for $L_{1}<x<L_{2}$ and $t>0$.

Theorem 6.13 Let the random initial condition $\left\{\phi(x): L_{1} \leq x \leq L_{2}\right\}$ be a process in $\mathrm{L}^{2}\left(\left[L_{1}, L_{2}\right] \times \Omega\right)$. Suppose that $\alpha^{2}, A_{1}$ and $\left(A_{2}, \ldots, \overline{A_{N}}\right)$ are independent and absolutely continuous, for $N \geq 2$ (recall that $A_{n}$ is defined in (6.3) as the random Fourier coefficient of $\psi$, where $\psi$ is defined from $\phi$ in relation (6.11)). Suppose that the probability density function $f_{A_{1}}$ is a.e. continuous on $\mathbb{R}$ and $\left\|f_{A_{1}}\right\|_{\mathrm{L}^{\infty}(\mathbb{R})}<\infty$. Assume that $\mathbb{E}\left[\mathrm{e}^{\pi^{2} \alpha^{2} t /\left(L_{2}-L_{1}\right)^{2}}\right]<\infty$. Then the sequence defined by (6.17) where $f_{v_{N}}$ is the density defined by (6.5), converges pointwise to the density $f_{u(x, t)}(u)$ of the solution stochastic process $u(x, t)(\omega)$ to the randomized heat equation (6.8) with deterministic boundary conditions $A$ and $B$, for $L_{1}<x<L_{2}$ and $t>0$.

\subsection{Approximation of the expectation and variance of the solution stochastic process}

By Theorem 6.1 ii), if $\psi \in \mathrm{L}^{2}([0,1] \times \Omega)$ (this holds if $\phi \in \mathrm{L}^{2}\left(\left[L_{1}, L_{2}\right] \times \Omega\right)$ and $A, B \in \mathrm{L}^{2}(\Omega)$, by 6.12$)$ and $0<a \leq \beta^{2}(\omega) \leq b$ a.s., then $v_{N}(y, t) \rightarrow v(y, t)$ in $\mathrm{L}^{2}(\Omega)$ as $N \rightarrow \infty$. 
In fact, looking at the proof of [16, Th. 1.3], we can be more precise: in that proof, it was shown that $\left\|A_{n}\right\|_{\mathrm{L}^{2}(\Omega)} \leq C$, for all $n$. If we assume that $\beta^{2}$ and $A_{n}$ are independent, for each $n$, and that $\sum_{n=1}^{\infty}\left\|\mathrm{e}^{-n^{2} \pi^{2} \beta^{2} t}\right\|_{\mathrm{L}^{2}(\Omega)}<\infty$, then

$$
\begin{aligned}
\sum_{n=1}^{\infty}\left\|A_{n} \mathrm{e}^{-n^{2} \pi^{2} \beta^{2} t} \sin (n \pi y)\right\|_{\mathrm{L}^{2}(\Omega)} & \leq \sum_{n=1}^{\infty}\left\|A_{n}\right\|_{\mathrm{L}^{2}(\Omega)}\left\|\mathrm{e}^{-n^{2} \pi^{2} \beta^{2} t}\right\|_{\mathrm{L}^{2}(\Omega)} \\
& \leq C \sum_{n=1}^{\infty}\left\|\mathrm{e}^{-n^{2} \pi^{2} \beta^{2} t}\right\|_{\mathrm{L}^{2}(\Omega)}<\infty,
\end{aligned}
$$

which implies that $v_{N}(y, t) \rightarrow v(y, t)$ in $\mathrm{L}^{2}(\Omega)$ as $N \rightarrow \infty$. By (6.9) and (6.14), this is equivalent to $u_{N}(x, t) \rightarrow u(x, t)$ in $\mathrm{L}^{2}(\Omega)$ as $N \rightarrow \infty$.

We already know that, if $v_{N}(y, t)(\omega), A(\omega)$ and $B(\omega)$ are absolutely continuous and independent random variables, then $u_{N}(x, t)(\omega)$ has a density function $f_{u_{N}(x, t)}(u)$ given by (6.15). On the other hand, if $A$ and $B$ are deterministic, assuming that $v_{N}(y, t)(\omega)$ is absolutely continuous one has that $u_{N}(x, t)(\omega)$ has a density function $f_{u_{N}(x, t)}(u)$ expressed by 6.17). Thus,

$$
\mathbb{E}\left[u_{N}(x, t)\right]=\int_{\mathbb{R}} u f_{u_{N}(x, t)}(u) \mathrm{d} u
$$

and

$$
\mathbb{V}\left[u_{N}(x, t)\right]=\int_{\mathbb{R}} u^{2} f_{u_{N}(x, t)}(u) \mathrm{d} u-\left(\mathbb{E}\left[u_{N}(x, t)\right]\right)^{2} .
$$

We summarize these ideas in the following theorem and remark, where the random or deterministic nature of the parameters $A$ and $B$ is distinguished, respectively, for the sake of completeness in the statement of our findings:

Theorem 6.14 If $\phi \in \mathrm{L}^{2}\left(\left[L_{1}, L_{2}\right] \times \Omega\right), A, B \in \mathrm{L}^{2}(\Omega), \alpha^{2},\left(A_{1}, \ldots, A_{N}\right), A, B$ are absolutely continuous and independent, $\sum_{n=1}^{\infty}\left\|\mathrm{e}^{-n^{2} \pi^{2} \alpha^{2} t /\left(L_{2}-L_{1}\right)^{2}}\right\|_{\mathrm{L}^{2}(\Omega)}<$ $\infty$, then $u(x, t) \in \mathrm{L}^{2}(\Omega)$,

$$
\begin{gathered}
\mathbb{E}\left[u_{N}(x, t)\right]=\int_{\mathbb{R}} u f_{u_{N}(x, t)}(u) \mathrm{d} u \stackrel{N \rightarrow \infty}{\longrightarrow} \mathbb{E}[u(x, t)], \\
\mathbb{V}\left[u_{N}(x, t)\right]=\int_{\mathbb{R}} u^{2} f_{u_{N}(x, t)}(u) \mathrm{d} u-\left(\mathbb{E}\left[u_{N}(x, t)\right]\right)^{2} \stackrel{N \rightarrow \infty}{\longrightarrow} \mathbb{V}[u(x, t)],
\end{gathered}
$$

for each $L_{1}<x<L_{2}$ and $t>0$.

Remark 6.15 Theorem 6.14 holds in the case that $A$ and $B$ are deterministic values. 


\subsection{Applications}

The first question that arises is to which random diffusion coefficients, random boundary conditions and random initial conditions our results can be applied.

We begin by studying hypothesis

$$
\sum_{n=m}^{\infty}\left\|\mathrm{e}^{-\left(n^{2}-2\right) \pi^{2} \alpha^{2} t /\left(L_{2}-L_{1}\right)^{2}}\right\|_{\mathrm{L}^{1}(\Omega)}<\infty,
$$

for $t>0$. It is clear that if $\alpha^{2}$ is bounded below, meaning that $\alpha^{2}(\omega) \geq a>0$ for a.e. $\omega \in \Omega$, then $(6.20)$ holds. This covers all cases in practice, as we may truncate $\alpha^{2}$, 84]. Notice, however, that the condition $\alpha^{2}(\omega) \geq a>0$ is not necessary to have (6.20). For example, if $\alpha^{2} \sim \operatorname{Uniform}(0, b), b>0$, then we know that its moment generating function is given by

$$
\mathbb{E}\left[\mathrm{e}^{\lambda \alpha^{2}}\right]=\frac{\mathrm{e}^{\lambda b}-1}{\lambda b}
$$

therefore

$$
\begin{aligned}
\sum_{n=m}^{\infty}\left\|\mathrm{e}^{-\left(n^{2}-2\right) \pi^{2} \alpha^{2} t /\left(L_{2}-L_{1}\right)^{2}}\right\|_{\mathrm{L}^{1}(\Omega)} & =\sum_{n=m}^{\infty} \frac{\mathrm{e}^{-\left(n^{2}-2\right) \pi^{2} b t /\left(L_{2}-L_{1}\right)^{2}}-1}{-\left(n^{2}-2\right) \pi^{2} b t /\left(L_{2}-L_{1}\right)^{2}} \\
& \leq \sum_{n=m}^{\infty} \frac{1}{\left(n^{2}-2\right) \pi^{2} b t /\left(L_{2}-L_{1}\right)^{2}}<\infty
\end{aligned}
$$

Another distribution for which $(6.20)$ holds, this time not upper-bounded, is

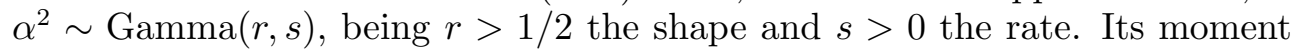
generating function is given by $\mathbb{E}\left[\mathrm{e}^{\lambda \alpha^{2}}\right]=1 /\left(1-\frac{\lambda}{s}\right)^{r}$, for $\lambda<s$. Then

$$
\sum_{n=m}^{\infty}\left\|\mathrm{e}^{-\left(n^{2}-2\right) \pi^{2} \alpha^{2} t /\left(L_{2}-L_{1}\right)^{2}}\right\|_{\mathrm{L}^{1}(\Omega)}=\sum_{n=m}^{\infty} \frac{1}{\left[\left(1+\left(n^{2}-2\right) \pi^{2} t /\left(s\left(L_{2}-L_{1}\right)^{2}\right)\right]^{r}\right.}<\infty .
$$

Notice that, if $0<r \leq 1 / 2$, then $\sum_{n=m}^{\infty}\left\|\mathrm{e}^{-\left(n^{2}-2\right) \pi^{2} \alpha^{2} t /\left(L_{2}-L_{1}\right)^{2}}\right\|_{\mathrm{L}^{1}(\Omega)}=\infty$. This shows that hypothesis 6.20 might not hold.

Concerning hypothesis

$$
\mathbb{E}\left[\mathrm{e}^{\pi^{2} \alpha^{2} t /\left(L_{2}-L_{1}\right)^{2}}\right]<\infty,
$$

for $t>0$, just take any distribution with finite moment generating function for $t>0$. For instance, Uniform $(0, b)$ with moment generating function (6.21), $\operatorname{Normal}\left(\mu, \sigma^{2}\right)$ with moment generating function at $\lambda$ given by $\mathrm{e}^{\mu \lambda+1 / 2 \sigma^{2} \lambda^{2}}$, etc. 
The gamma distribution may be used to highlight the fact that, fixed $t>0$, hypotheses $(6.20)$ and $(6.23)$ are independent. Suppose that $\alpha^{2} \sim \operatorname{Gamma}(r, s)$, being $r>0$ the shape and $s>0$ the rate. Then (6.20) is accomplished if and only if $r>1 / 2$ and $s>0$ (see (6.22), whereas (6.23) fulfills if and only if $r>0$ and $\pi^{2} t /\left(L_{2}-L_{1}\right)^{2}<s$.

The most difficult step is to compute $f_{A_{1}}$ and $f_{\left(A_{2}, \ldots, A_{N}\right)}$ in 6.5). We are going to see that the density function of $A_{n}(\omega)=2 \int_{0}^{1} \psi(y)(\omega) \sin (n \pi y) \mathrm{d} y$ can be computed when the initial condition process $\phi$ has a certain expression concerning the Karhunen-Loève expansion [89, Th. 5.28]. Take $\psi$ defined in (6.11). As $\psi \in \mathrm{L}^{2}([0,1] \times \Omega)$, for each fixed $\omega \in \Omega$ the real function $\psi(\cdot)(\omega)$ belongs to $\mathrm{L}^{2}([0,1])$. We can expand $\psi(\cdot)(\omega)$ as a Fourier series on $[0,1]$ with the orthonormal basis $\{\sqrt{2} \sin (j \pi y)\}_{j=1}^{\infty}$. Hence,

$$
\psi(y)(\omega)=\sum_{j=1}^{\infty} c_{j}(\omega) \sqrt{2} \sin (j \pi y),
$$

where the series is taken in $\mathrm{L}^{2}([0,1])$ for each $\omega \in \Omega$, and where $c_{j}(\omega)$ are the random variables corresponding to the Fourier coefficients of $\psi(\cdot)(\omega)$. This expression (6.24) corresponds to the Karhunen-Loève expansion of the process $\psi$. We will restrict to processes for which the random Fourier coefficients $\left\{c_{j}\right\}_{j=1}^{\infty}$ are independent and absolutely continuous random variables. Thus, we write

$$
\psi(y)(\omega)=\sum_{j=1}^{\infty} \sqrt{\nu_{j}} \sqrt{2} \sin (j \pi y) \xi_{j}(\omega),
$$

where the series converges in $\mathrm{L}^{2}([0,1] \times \Omega),\left\{\nu_{j}\right\}_{j=1}^{\infty}$ are nonnegative real numbers satisfying $\sum_{j=1}^{\infty} \nu_{j}<\infty$ and $\left\{\xi_{j}\right\}_{j=1}^{\infty}$ are absolutely continuous random variables with zero expectation, unit variance and independent $\left(c_{j}(\omega)=\right.$ $\sqrt{\nu_{j}} \xi_{j}(\omega)$, so that $\xi_{j}$ standardizes $\left.c_{j}(\omega)\right)$. Notice that the sum is well-defined in $\mathrm{L}^{2}([0,1] \times \Omega)$, because for two indexes $N>M$ we have, by Pythagoras's Theorem in $\mathrm{L}^{2}([0,1] \times \Omega)$,

$$
\begin{aligned}
\left\|\sum_{j=M+1}^{N} \sqrt{\nu_{j}} \sqrt{2} \sin (j \pi x) \xi_{j}\right\|_{\mathrm{L}^{2}([0,1] \times \Omega)}^{2} & =\sum_{j=M+1}^{N} \nu_{j}\|\sqrt{2} \sin (j \pi x)\|_{\mathrm{L}^{2}([0,1])}^{2}\left\|\xi_{j}\right\|_{\mathrm{L}^{2}(\Omega)}^{2} \\
& =\sum_{j=M+1}^{N} \nu_{j} \stackrel{N, M \rightarrow \infty}{\longrightarrow} 0 .
\end{aligned}
$$


We can compute explicitly the random Fourier coefficients $A_{n}$ :

$$
\begin{aligned}
A_{n}(\omega) & =2 \int_{0}^{1} \psi(y)(\omega) \sin (n \pi y) \mathrm{d} y \\
& =2 \sum_{j=1}^{\infty} \sqrt{\nu_{j}} \sqrt{2} \int_{0}^{1} \sin (j \pi y) \sin (n \pi y) \mathrm{d} y \xi_{j}(\omega) \\
& =\sqrt{2} \sqrt{\nu_{n}} \xi_{n}(\omega) .
\end{aligned}
$$

The key fact in this computation is that the eigenfunctions of the SturmLiouville problem associated to 6.1 are precisely $\{\sqrt{2} \sin (j \pi y)\}_{j=1}^{\infty}$. From (6.27) and our assumptions on $\left\{\xi_{j}\right\}_{j=1}^{\infty}$, we derive that $A_{1}, A_{2}, \ldots$ are independent and absolutely continuous random variables. Using the Random Variable Transformation technique,

$$
f_{A_{n}}(a)=\frac{1}{\sqrt{2 \nu_{n}}} f_{\xi_{n}}\left(\frac{a}{\sqrt{2 \nu_{n}}}\right) .
$$

If $f_{\xi_{1}}$ is Lipschitz (respectively a.e. continuous and essentially bounded) on $\mathbb{R}$, then $f_{A_{1}}$ is Lipschitz (respectively a.e. continuous and essentially bounded) on $\mathbb{R}$ too, and all the hypotheses of Theorem 6.10 (respectively Theorem 6.11) are fulfilled.

The Lipschitz condition on $\mathbb{R}$ is satisfied by the probability density function of some named distributions:

- $\operatorname{Normal}\left(\mu, \sigma^{2}\right), \mu \in \mathbb{R}$ and $\sigma^{2}>0$.

- $\operatorname{Beta}(a, b), a, b \geq 2$.

- $\operatorname{Gamma}(a, b), a \geq 2$ and $b>0$.

In general, any density with bounded derivative on $\mathbb{R}$ satisfies the Lipschitz condition on $\mathbb{R}$, by the Mean Value Theorem. By contrast, some non-Lipschitz density functions are the uniform distribution, the exponential distribution, etc. or any other density with a jump discontinuity at some point of $\mathbb{R}$. However, non-Lipschitz density functions may be regularized at the point of discontinuity so that the Lipschitz assumption is fulfilled and, moreover, the probabilistic behavior of the regularized density function is the same in practice as the original non-Lipschitz density.

The a.e. continuity and essential boundedness is satisfied by the probability density function of more distributions: 
- $\operatorname{Normal}\left(\mu, \sigma^{2}\right), \mu \in \mathbb{R}$ and $\sigma^{2}>0$.

- $\operatorname{Beta}(a, b), a, b \geq 1$.

- Uniform $(a, b), a<b$.

- $\operatorname{Gamma}(a, b), a \geq 1$ and $b>0$. In particular, Exponential $(\lambda), \lambda>0$.

- Truncated normal distribution.

We will do examples for initial conditions $\phi$ such that the corresponding $\psi$ is written as (6.25), being $\xi_{1}, \xi_{2}, \ldots$ independent and absolutely continuous random variables, with zero expectation and unit variance, $f_{\xi_{1}}$ Lipschitz on $\mathbb{R}$ and $\sum_{j=1}^{\infty} \nu_{j}<\infty$. In the examples, we will combine $A$ and $B$ deterministic and absolutely continuous random variables, with $\psi$ being a Gaussian and non-Gaussian process. Hence, all the examples suppose an improvement of [16.

The densities $f_{u_{N}(x, t)}(u)$ that approximate $f_{u(x, t)}(u)$ will be computed numerically in an (almost) exact manner, using the software Mathematica ${ }^{\circledR}$, concretely, its built-in function NIntegrate. In this way, we will be able to study the exact difference between two consecutive orders of truncation $N$ and $N+1$.

\section{Example 6.16 ( $\psi$ Gaussian, $A$ and $B$ deterministic) Let}

$$
\psi(y)(\omega)=\sum_{j=1}^{\infty} \frac{\sqrt{2}}{\pi j} \sin (j \pi y) \xi_{j}(\omega)
$$

be a standard Brownian bridge on $[0,1]$, see [89, Example 5.30], being $\xi_{1}, \xi_{2}, \ldots$ independent and $\operatorname{Normal}(0,1)$ random variables. By (6.11), $\phi$ is a Brownian bridge on $\left[L_{1}, L_{2}\right]$ that takes the values $A$ and $B$ at the boundary. We choose $L_{1}=0$ and $L_{2}=6, A=-3$ and $B=3$. The diffusion coefficient is $\alpha^{2} \sim$ Uniform $(1,2)$. Theorem 6.12 applies in this case.

In Figure 6.1, three plots of the path described by $\phi(x)$ for three different outcomes $\omega$ are shown.

In Figure 6.2, we approximate the probability density function of the solution stochastic process $u(x, t)(\omega)$ at $x=5$ and $t=0.2$, using (6.17), for $N=$ $1,2,3,4$. Convergence seems to be achieved for $N=3$ and $N=4$. In Table6.1. the infinity norm of the difference of two consecutive orders of approximation $N$ and $N+1$, for $N=1,2,3$, is computed. We can see that the errors decrease to 0 as $N$ grows, which agrees with our theoretical findings. In Table 6.2 

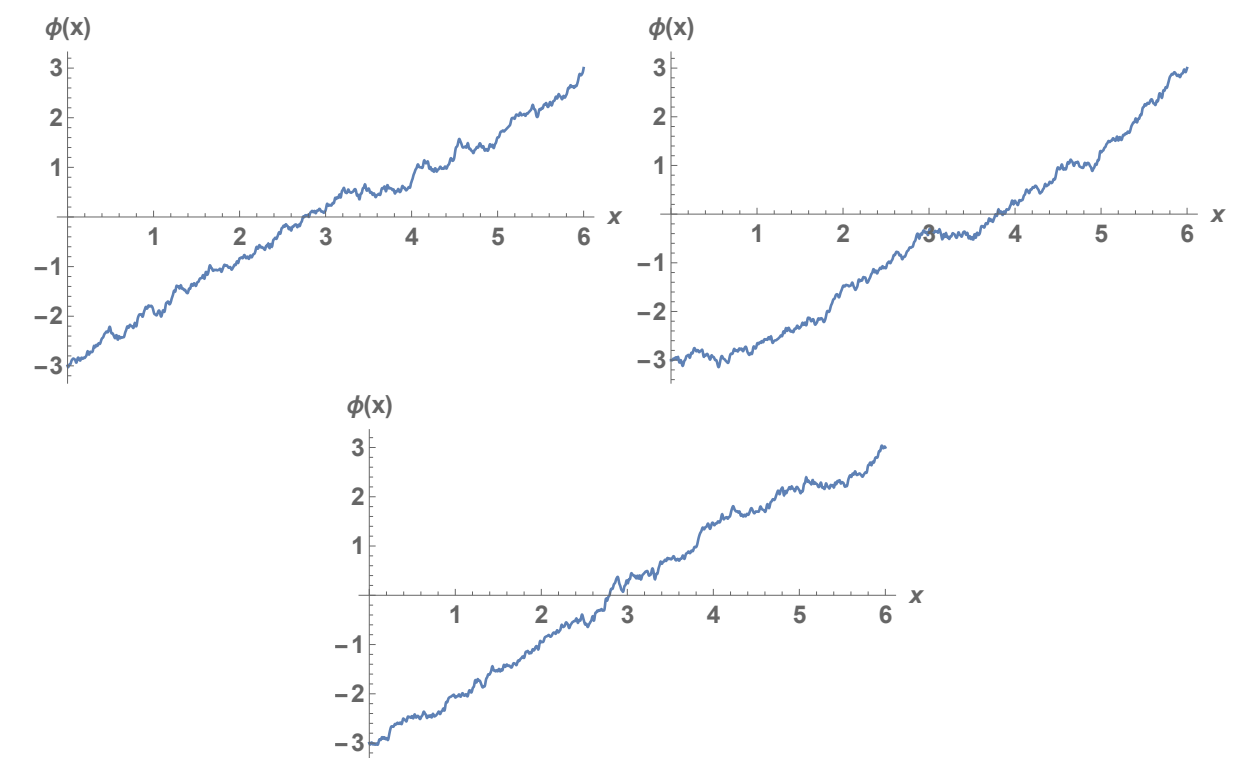

Figure 6.1: Paths of the initial condition $\phi(x)$ for three different outcomes $\omega$. Example 6.16

using Theorem 6.14 together with Remark 6.15, the expectation and variance of $u(x, t)(\omega)$ have been approximated, for different orders of truncation.

\begin{tabular}{|c|c|}
\hline$N$ & $\left\|f_{u_{N}(5,0.2)}-f_{u_{N+1}(5,0.2)}\right\|_{L^{\infty}(\mathbb{R})}$ \\
\hline 1 & 0.330855 \\
\hline 2 & 0.0622449 \\
\hline 3 & 0.00820879 \\
\hline
\end{tabular}

Table 6.1: Difference of two consecutive orders of approximation $N$ and $N+1$ given by 6.17 , for $N=1,2,3$. Example 6.16

\begin{tabular}{|c|c|c|c|c|}
\hline$N$ & 1 & 2 & 3 & 4 \\
\hline $\mathbb{E}\left[u_{N}(5,0.2)\right]$ & 2 & 2 & 2 & 2 \\
\hline $\mathbb{V}\left[u_{N}(5,0.2)\right]$ & 0.0429981 & 0.0628341 & 0.0681679 & 0.0689422 \\
\hline
\end{tabular}

Table 6.2: Approximations of $\mathbb{E}[u(5,0.2)]$ and $\mathbb{V}[u(5,0.2)]$ for $N=1,2,3,4$ constructed by 6.18 and 6.19), respectively, being $f_{u_{N}(x, t)}$ given by (6.17). Example6.16 


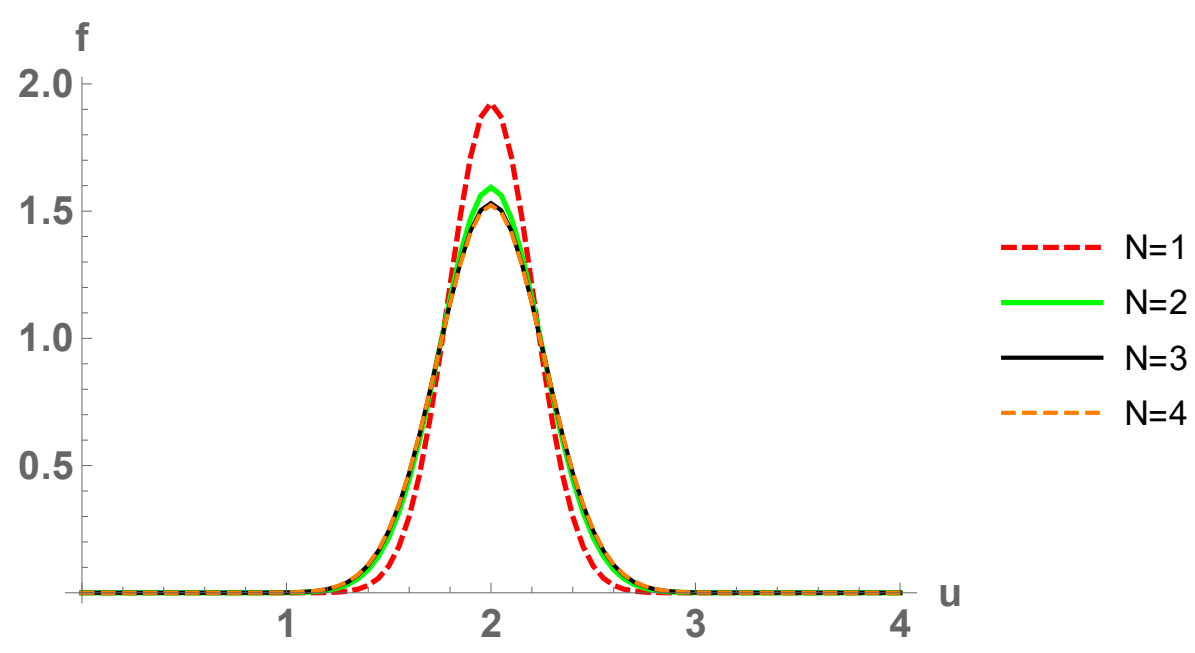

Figure 6.2: Approximation 6.17 for $N=1,2,3,4$ at $(x, t)=(5,0.2)$. Example 6.16

Example 6.17 ( $\psi$ non-Gaussian, $A$ and $B$ deterministic) Let

$$
\psi(y)(\omega)=\sum_{j=1}^{\infty} \frac{\sqrt{2}}{j^{\frac{3}{2}} \sqrt{1+\log j}} \sin (j \pi y) \xi_{j}(\omega),
$$

where $\xi_{1}, \xi_{2}, \ldots$ are independent and identically distributed random variables with density function

$$
f_{\xi_{1}}(\xi)=\frac{\sqrt{2}}{\pi\left(1+\xi^{4}\right)}, \quad-\infty<\xi<\infty .
$$

It is easy to check that this is indeed a density function, with zero expectation and unit variance. Thereby, $\psi$ is a non-Gaussian stochastic process on $[0,1]$ (if it were Gaussian, then $\xi_{1}, \xi_{2}, \ldots$ would be normally distributed, see [89, Th. 5.28]). The sum defining $\psi$ is well-defined in $\mathrm{L}^{2}([0,1] \times \Omega)$, because $\sum_{j=1}^{\infty} 1 /\left(j^{3}(1+\log j)\right)<\infty($ see 6.26) $)$. By 6.11), we can simulate the sample paths of $\phi(x)$ on $\left[L_{1}, L_{2}\right]$. The data chosen are $L_{1}=-8, L_{2}=2 \pi+1, A=-1$ and $B=2$. The distribution for $\alpha^{2}$ is Uniform $(1,2)$. Theorem 6.12 guarantees the convergence of the approximating sequence 6.17.

In Figure 6.3, three plots of the path described by $\phi(x)$ for three different outcomes $\omega$ are presented. 


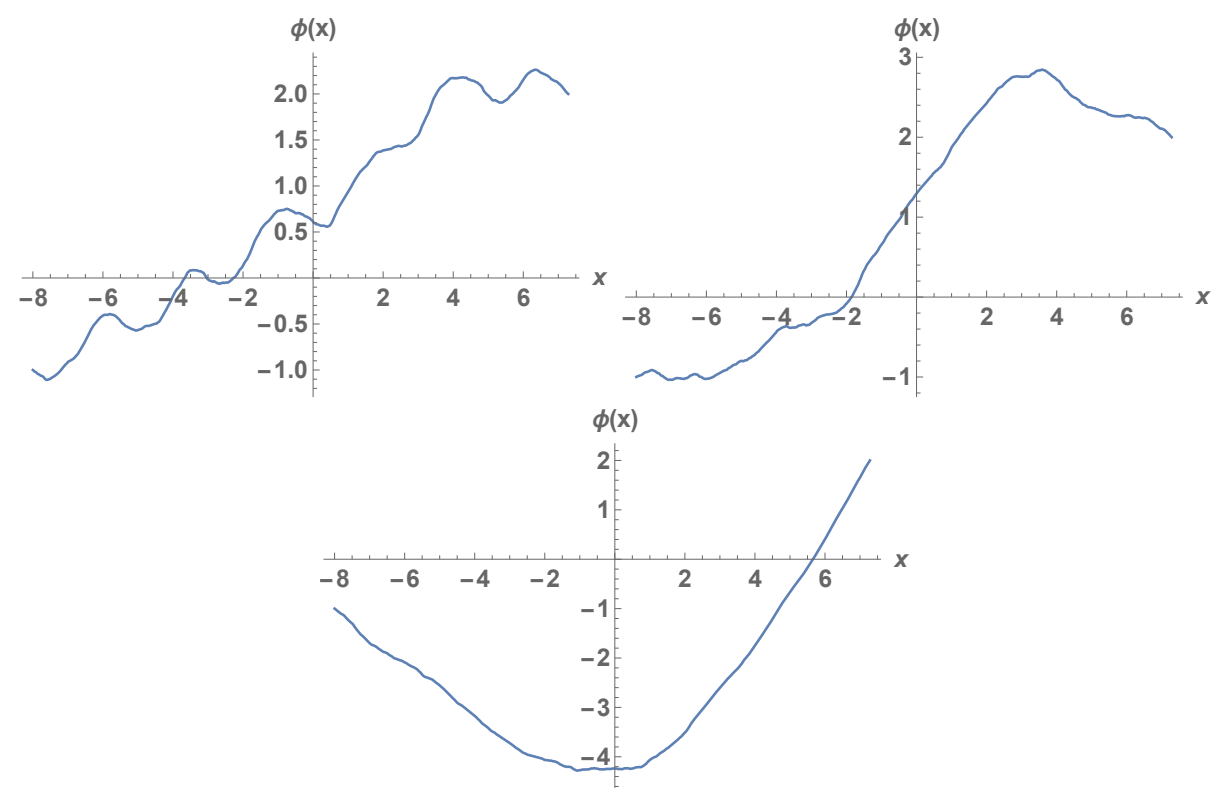

Figure 6.3: Paths of the initial condition $\phi(x)$ for three different outcomes $\omega$. Example 6.17

In Figure 6.4, we approximate the probability density function of the solution stochastic process $u(x, t)(\omega)$ at $x=1$ and $t=0.1$, using (6.17), for $N=1,2,3,4$. In order to assess convergence analytically, in Table 6.3, the maximum of the difference of two consecutive orders of approximation $N$ and $N+1$ given by (6.17), for $N=1,2,3$, is computed. The errors decrease to 0 as $N$ grows, which goes in the direction of our theoretical results. In Table 6.4 the expectation and variance of $u(x, t)(\omega)$ have been approximated, using Theorem 6.14 and Remark 6.15 together with expressions (6.18) and 6.19, respectively.

\begin{tabular}{|c|c|}
\hline$N$ & $\left\|f_{u_{N}(1,0.1)}-f_{u_{N+1}(1,0.1)}\right\|_{L^{\infty}(\mathbb{R})}$ \\
\hline 1 & 0.00631990 \\
\hline 2 & 0.00214919 \\
\hline 3 & 0.00128318 \\
\hline
\end{tabular}

Table 6.3: Difference of two consecutive orders of approximation $N$ and $N+1$ given by 6.17, for $N=1,2,3$. Example 6.17. 


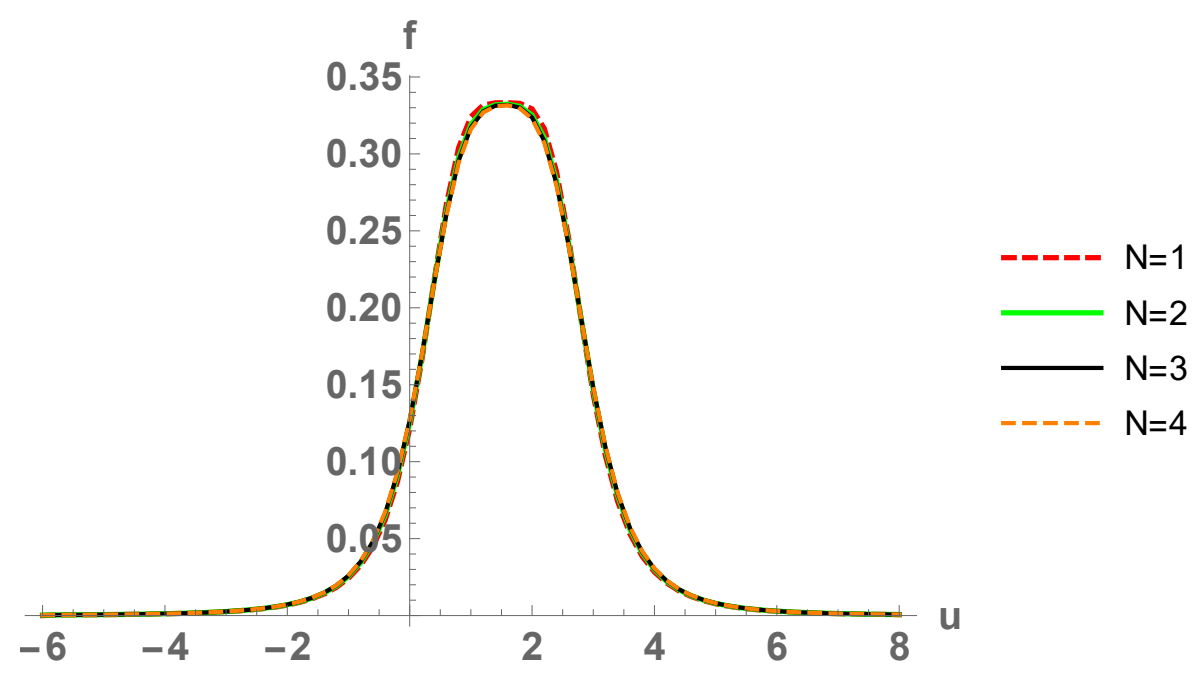

Figure 6.4: Approximation 6.17) for $N=1,2,3,4$ at $(x, t)=(1,0.1)$. Example 6.17

\begin{tabular}{|c|c|c|c|c|}
\hline$N$ & 1 & 2 & 3 & 4 \\
\hline $\mathbb{E}\left[u_{N}(1,0.1)\right]$ & 1.54545 & 1.54497 & 1.54495 & 1.54494 \\
\hline $\mathbb{V}\left[u_{N}(1,0.1)\right]$ & 1.51182 & 1.55304 & 1.56661 & 1.57496 \\
\hline
\end{tabular}

Table 6.4: Approximations of $\mathbb{E}[u(1,0.1)]$ and $\mathbb{V}[u(1,0.1)]$ for $N=1,2,3,4$ constructed by 6.18 and 6.19, respectively, being $f_{u_{N}(x, t)}$ given by 6.17. Example 6.17. 
Example 6.18 ( $\psi$ Gaussian, $A$ and $B$ random) Let

$$
\psi(y)(\omega)=\sum_{j=1}^{\infty} \frac{\sqrt{2}}{\pi j} \sin (j \pi y) \xi_{j}(\omega)
$$

be a standard Brownian bridge on $[0,1]$, as in Example 6.16. The data chosen are $L_{1}=0, L_{2}=6$ and $\alpha^{2} \sim$ Uniform $(1,2)$, as in Example 6.16, but now the boundary conditions $A$ and $B$ are random: $A$ follows a triangular distribution with ends -5 and -2 and mode -3 , whereas $B$ is an exponentially distributed random variable with mean 2 and truncated to $[3,5]$. The modes of $A$ and $B$ coincide with the deterministic boundary conditions in Example 6.16, so similar results for the density function could occur.

In Figure 6.5, three plots of the path described by $\phi(x)$ for three different outcomes $\omega$ are presented.

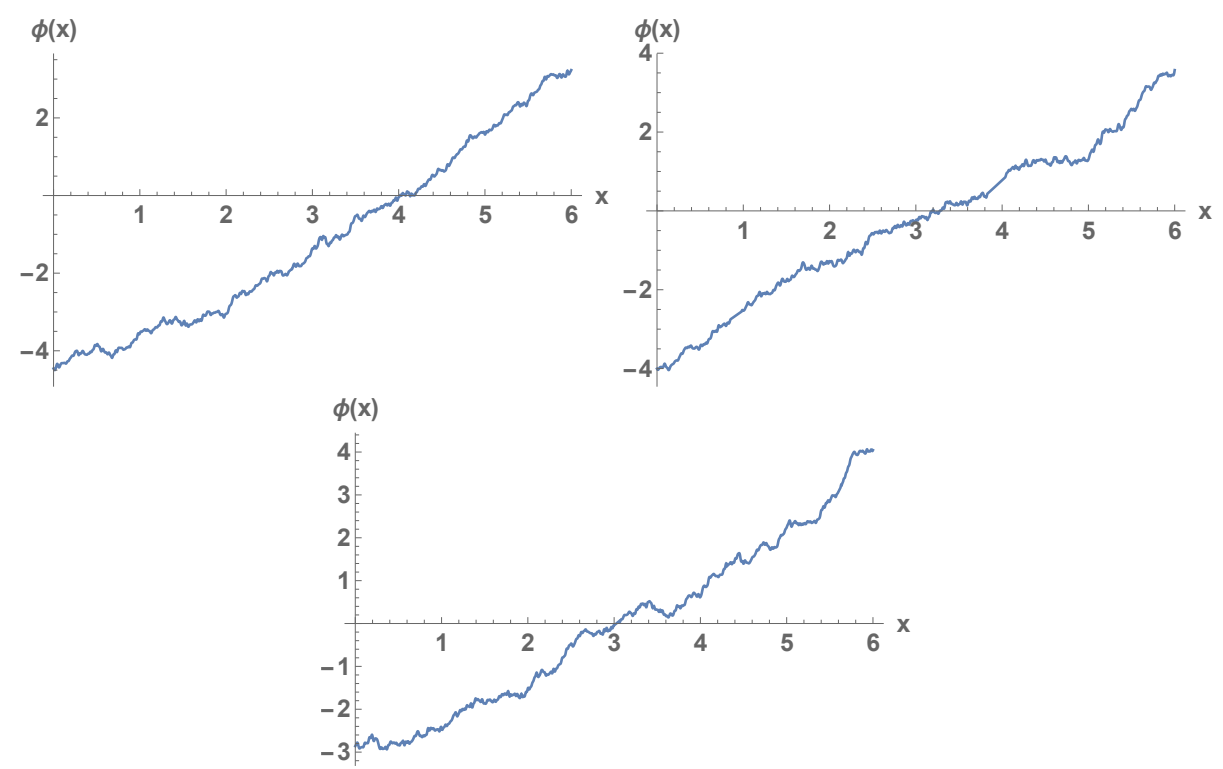

Figure 6.5: Paths of the initial condition $\phi(x)$ for three different outcomes $\omega$. Example 6.18

In Figure 6.6, we approximate the probability density function of the solution stochastic process $u(x, t)(\omega)$ at $x=5$ and $t=0.2$, using 6.15), for $N=$ $1,2,3,4$. Compare the plots with those of Example 6.16, where the boundary conditions were deterministic with constant value the mode of $A$ and $B$. In 
Table 6.5, the errors are analyzed. In Table 6.6, both $\mathbb{E}[u(x, t)]$ and $\mathbb{V}[u(x, t)]$ are approximated, according to Theorem 6.14.

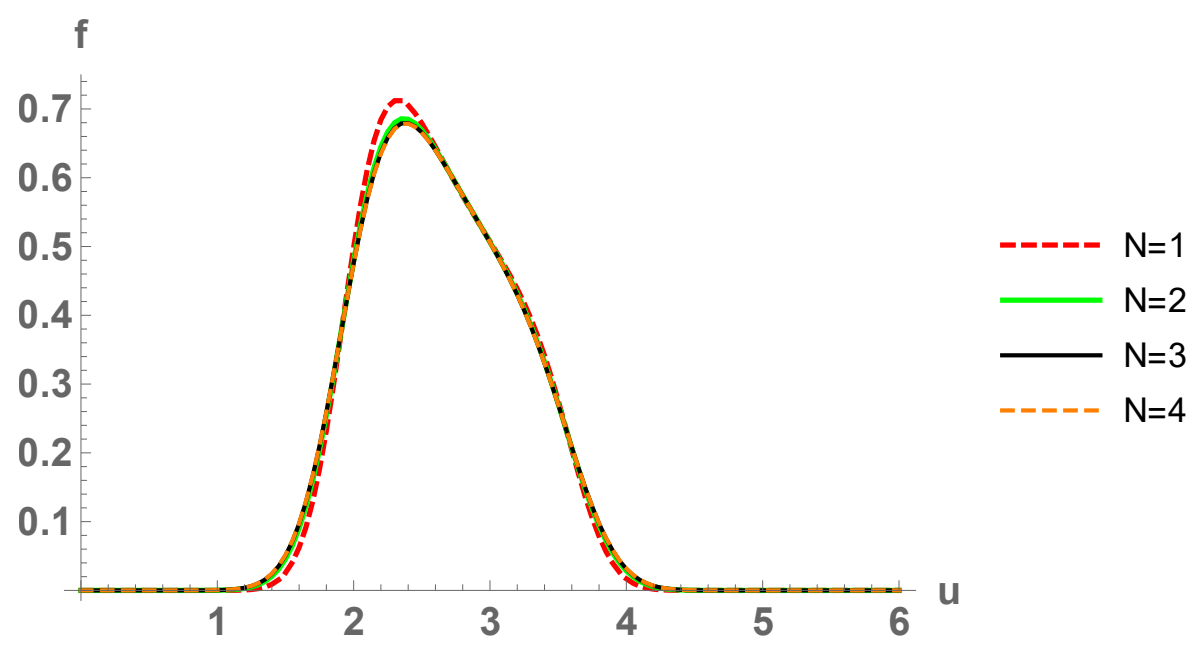

Figure 6.6: Approximation 6.15 for $N=1,2,3,4$ at $(x, t)=(5,0.2)$. Example 6.18

\begin{tabular}{|c|c|}
\hline$N$ & $\left\|f_{u_{N}(5,0.2)}-f_{u_{N+1}(5,0.2)}\right\|_{L^{\infty}(\mathbb{R})}$ \\
\hline 1 & 0.0390501 \\
\hline 2 & 0.00870084 \\
\hline 3 & 0.00119532 \\
\hline
\end{tabular}

Table 6.5: Difference of two consecutive orders of approximation $N$ and $N+1$ given by 6.15 , for $N=1,2,3$. Example 6.18.

\begin{tabular}{|c|c|c|c|c|}
\hline$N$ & 1 & 2 & 3 & 4 \\
\hline $\mathbb{E}\left[u_{N}(5,0.2)\right]$ & 2.64115 & 2.64115 & 2.64115 & 2.64115 \\
\hline $\mathbb{V}\left[u_{N}(5,0.2)\right]$ & 0.274152 & 0.293988 & 0.299323 & 0.300101 \\
\hline
\end{tabular}

Table 6.6: Approximations of $\mathbb{E}[u(5,0.2)]$ and $\mathbb{V}[u(5,0.2)]$ for $N=1,2,3,4$ constructed by 6.18 and 6.19, respectively, being $f_{u_{N}(x, t)}$ given by 6.15. Example 6.18.

Example 6.19 ( $\psi$ non-Gaussian, $A$ and $B$ random) Let

$$
\psi(y)(\omega)=\sum_{j=1}^{\infty} \frac{\sqrt{2}}{j^{\frac{3}{2}} \sqrt{1+\log j}} \sin (j \pi y) \xi_{j}(\omega),
$$


be the same process as in Example 6.17. The interval where the heat equation is defined has endpoints $L_{1}=-8$ and $L_{2}=2 \pi+1$, and $\alpha^{2} \sim \operatorname{Uniform}(1,2)$, as in Example 6.17. But now the boundary conditions $A$ and $B$ are random (with no constant values for the modes of $\mathrm{A}$ and $\mathrm{B}): A \sim \operatorname{Uniform}(-1.5,-0.5)$ and $B \sim \operatorname{Normal}(2,1)$. Notice that $\mathbb{E}[A]$ and $\mathbb{E}[B]$ are the deterministic boundary conditions of Example 6.17, so the approximated density functions may resemble those from Example 6.17.

In Figure 6.7, three plots of the path described by $\phi(x)$ for three different outcomes $\omega$ are presented.

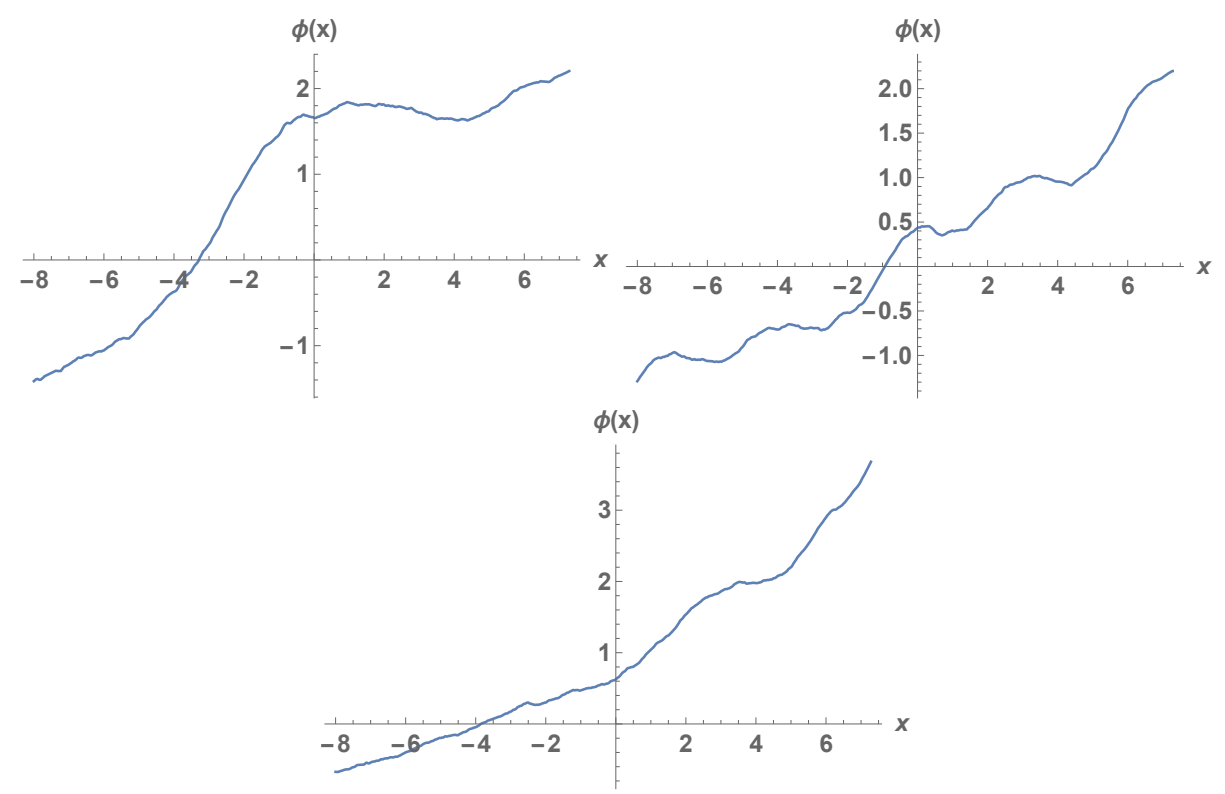

Figure 6.7: Paths of the initial condition $\phi(x)$ for three different outcomes $\omega$. Example 6.19

In Figure 6.8, we approximate the probability density function of the solution stochastic process $u(x, t)(\omega)$ at $x=1$ and $t=0.1$, using $(6.15)$, for $N=1,2,3,4$. These plots are very similar to those from Example 6.17. This occurs because the expectation of our random boundary conditions $A$ and $B$ is equal to the deterministic boundary conditions of Example 6.17. In Table 6.7. we present the errors between two consecutive orders of approximation. The expectation and variance of the solution process $u(x, t)(\omega)$ have been approximated in Table 6.8, based on Theorem 6.14. 


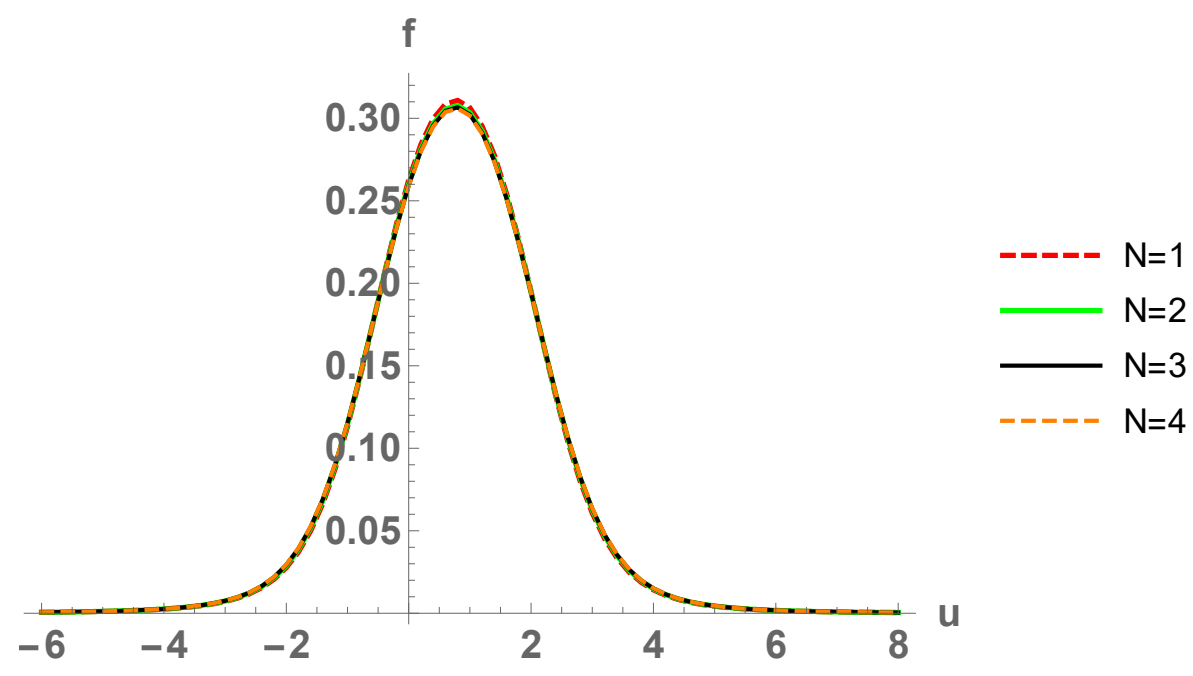

Figure 6.8: Approximation 6.15 for $N=1,2,3,4$ at $(x, t)=(1,0.1)$. Example 6.19

\begin{tabular}{|c|c|}
\hline$N$ & $\left\|f_{u_{N}(1,0.1)}-f_{u_{N+1}(1,0.1)}\right\|_{\mathrm{L}^{\infty}(\mathbb{R})}$ \\
\hline 1 & 0.00303628 \\
\hline 2 & 0.00111420 \\
\hline 3 & 0.000685738 \\
\hline
\end{tabular}

Table 6.7: Difference of two consecutive orders of approximation $N$ and $N+1$ given by 6.15 , for $N=1,2,3$. Example 6.19.

\begin{tabular}{|c|c|c|c|c|}
\hline$N$ & 1 & 2 & 3 & 4 \\
\hline $\mathbb{E}\left[u_{N}(1,0.1)\right]$ & 0.766541 & 0.766546 & 0.766548 & 0.766545 \\
\hline $\mathbb{V}\left[u_{N}(1,0.1)\right]$ & 1.86628 & 1.90366 & 1.91720 & 1.92552 \\
\hline
\end{tabular}

Table 6.8: Approximations of $\mathbb{E}[u(1,0.1)]$ and $\mathbb{V}[u(1,0.1)]$ for $N=1,2,3,4$ constructed by 6.18 and 6.19, respectively, being $f_{u_{N}(x, t)}$ given by 6.15. Example 6.19. 


\subsection{Conclusions}

In this chapter we have determined approximations of the probability density function of the solution stochastic process to the randomized heat equation defined on a general bounded interval $\left[L_{1}, L_{2}\right]$ with non-homogeneous Dirichlet boundary conditions. We have reviewed results in the existing literature that establish conditions under which the probability density of the solution process to the random heat equation defined on $[0,1]$ with homogeneous Dirichlet boundary conditions can be approximated. By relating the solutions of the heat equation with homogeneous and non-homogeneous boundary conditions, and using the Random Variable Transformation technique, we have been able to set hypotheses on the random diffusion coefficient, on the random boundary conditions and on the initial condition process, so that the probability density function of the solution can be approximated uniformly or pointwise (Theorem 6.10, Theorem 6.11, Theorem 6.12 and Theorem 6.13). We have obtained results on the approximation of the expectation and variance of the solution (Theorem 6.14 and Remark 6.15).

Our theoretical findings have been applied to particular random heat equation problems on $\left[L_{1}, L_{2}\right]$ with non-homogeneous boundary conditions. We have dealt with random diffusion coefficients, with deterministic and random boundary conditions, and with initial condition processes having a certain Karhunen-Loève expansion, which may be Gaussian or may not. It has been evinced numerically that the convergence to the density function of the solution is achieved quickly.

\section{Acknowledgements}

This work has been supported by the Spanish Ministerio de Economía y Competitividad grant MTM2017-89664-P.

The main results of this chapter have been published in [19]. 



\section{Chapter 7}

\section{Probability density function approximation to the random heat PDE solution via a finite difference scheme}

We study the random heat partial differential equation on a bounded domain assuming that the diffusion coefficient and the Dirichlet boundary conditions are random variables, and the initial condition is a stochastic process. Under general conditions, this stochastic system possesses a unique solution stochastic process in the almost sure and mean square senses. To quantify the uncertainty for this solution process, the computation of the probability density function is a major goal. By using a finite difference scheme, we approximate the solution stochastic process at each point by a sequence of random variables, whose probability density functions are computable, i.e., we construct a sequence of approximating density functions. We show numerical examples that demonstrate the applicability of our approach and fast convergence. 


\subsection{Introduction and motivation}

Heat transfer modeling using partial differential equations has been extensively studied in the literature for many years, and it is currently an active field under research [63, 116]. Information such as the diffusion coefficient, the initial distribution of temperature, etc., appears in the mathematical formulation of this class of problems. In practice, this key information needs to be established via measurements, which often involve uncertainties from measurement errors, material impurities, etc. These facts have motivated the mathematical modeling of heat transfer by using random partial differential equations. This kind of differential equations are those in which input data (initial and boundary conditions, forcing term and coefficients) are conveniently treated as random variables and stochastic processes. As a consequence, the solution of a random partial differential equation is not a classical function but a stochastic process.

A powerful approach to deal with random partial differential equations is the so-called $\mathrm{L}^{p}$-random calculus, and in particular, the mean square random calculus corresponding to $p=2$, 125. Convergence in $\mathrm{L}^{2}$-random calculus is usually referred to as mean square (hereafter m.s.) convergence, [125, Ch. 4]. This approach has two key properties. The first one is the formal representation of the solution stochastic process, which coincides with the one of the deterministic case, i.e., when the random inputs are deterministic quantities. This permits retaining both the physical interpretation and the deterministic results via the random solution. The second one is a distinctive property of m.s. convergence regarding to other types of stochastic convergences (almost surely, hereafter a.s., in probability, and in distribution), which is crucial to compute reliable approximations of the mean and the variance of the solution stochastic process $u(x, t)$ (see [125, Th. 4.3.1]). If $u^{M}(x, t)$ is a sequence of random variables which is m.s. convergent to $u(x, t)$ as $M \rightarrow \infty$ for $(x, t)$ fixed, i.e., $u^{M}(x, t) \underset{M \rightarrow \infty}{\stackrel{\text { m.s. }}{\longrightarrow}} u(x, t)$, then

$$
\mathbb{E}\left[u^{M}(x, t)\right] \underset{M \rightarrow \infty}{\longrightarrow} \mathbb{E}[u(x, t)] \text { and } \mathbb{V}\left[u^{M}(x, t)\right] \underset{M \rightarrow \infty}{\longrightarrow} \mathbb{V}[u(x, t)] .
$$

In the context of heat transfer modeling via random partial differential equations, most of the contributions have focused on the construction of approximations of the solution stochastic process, and in the computation of its mean and variance as well. The calculation of these statistical moments is performed by taking advantage of the aforementioned key property of the m.s. convergence. Often, the methods and techniques that have been proposed to deal with random heat equations, are extensions of their deterministic counterpart. Further, some works are based upon analytical techniques [26, 47] 
while others rely on numerical methods [41, 48]. Thus, the heat equation on a bounded domain is studied by using random Fourier series, and assuming that the diffusion coefficient is a random variable, the initial condition is a stochastic process, and the boundary conditions are assumed to be null [47]. In another study, the analysis is performed on an unbounded domain, and it is assumed that the initial condition is given by a stochastic process [26]. Here, the key mathematical tool to conduct the study is the random Fourier integral transform. It has been also proposed a m.s. convergent numerical scheme to approximate the solution of random heat equation whose diffusion coefficient is a spatial-dependent stochastic process. The initial condition is described by a deterministic function and the boundary conditions are null [48. These results were extended for a general class of random diffusion equations [41]. As it has been indicated, in all those works approximations of the mean and the variance/standard deviation functions of the solution stochastic process are given. The computation of exact or approximate probability density function to random partial differential equations has been tackled in few contributions, since the majority of the studies are addressed to construct approximations of the mean and the variance of the solution stochastic process. Some exceptions include [57, 118, 122], for example.

In the present work, we will deal with the following heat partial differential equation on the spatial domain $[0,1]$ :

$$
\left\{\begin{array}{l}
u_{t}=\alpha u_{x x}, \quad x \in(0,1), t \in(0, T), \\
u(0, t)=A, \quad u(1, t)=B, t \in[0, T] \\
u(x, 0)=\phi(x), \quad x \in[0,1] .
\end{array}\right.
$$

For the sake of completeness, we recall that in a deterministic setting, the diffusion coefficient $\alpha>0$, and the boundary conditions $A$ and $B$ are constants, and the initial condition $\phi(x)$ is a deterministic function. Then, its solution is a bivariate function $u(x, t)$. Sufficient conditions for the existence of a smooth classical solution $u(x, t)$ are given from Theorem 3.1 in ref. [16].

Proposition 7.1 ([16, Th. 3.1]) If $\phi$ is continuous on $[0,1]$, piecewise $C^{1}$ on $[0,1], \phi(0)=A$ and $\phi(1)=B$, then $u(x, t)$ is continuous on $[0,1] \times[0, \infty)$, is of class $C^{2,1}$ on $(0,1) \times(0, \infty)$ and is a classical solution of (7.1).

Moreover, under the conditions of Proposition 7.1, the solution to (7.1) is given by

$$
u(x, t)=v(x, t)+x B+(1-x) A,
$$


where

$$
\begin{gathered}
v(x, t)=\sum_{k=1}^{\infty} A_{k} \mathrm{e}^{-k^{2} \pi^{2} \alpha t} \sin (k \pi x), \\
A_{k}=2 \int_{0}^{1} \psi(y) \sin (k \pi y) \mathrm{d} y, \quad \psi(y)=\phi(y)-y B-(1-y) A .
\end{gathered}
$$

This solution results from applying the classical method of separation of variables to (7.1) with homogeneous boundary conditions (case $A=B=0$ ), and then doing a change of variables to adapt to the case $A \neq 0$ or $B \neq 0$.

Motivated by the arguments exhibited at the beginning of this section, hereinafter we will consider the randomization of the above heat diffusion problem 7.1). We then assume that the values of the input data depend on an experiment $\omega$. The set of all experiments, called sample space and denoted by $\Omega$, is equipped with a $\sigma$-algebra of events $\mathcal{F}$, and a probability measure $\mathbb{P}$ to form a complete probability space $(\Omega, \mathcal{F}, \mathbb{P})$. The diffusion coefficient $\alpha=\alpha(\omega)$ and the boundary conditions $A=A(\omega)$ and $B=B(\omega)$ are random variables, and the initial condition $\phi(x)=\phi(x)(\omega)$ is assumed to be a stochastic process, being all of them defined in the probability space $(\Omega, \mathcal{F}, \mathbb{P})$. The term $u$ is a stochastic process $u(x, t)=u(x, t)(\omega)$ that solves the random heat diffusion problem (7.1) in some probabilistic sense. Theorem 3.2 in ref. [16] proves that under square integrability of $\phi, A$ and $B$, there is a unique solution stochastic process in the a.s. and m.s. senses [125]. Notice that, in this probabilistic scenario, we do not require $\phi(0)=A$ and $\phi(1)=B$.

Proposition 7.2 ([16, Th. 3.2]) The following statements hold:

i) Almost sure (a.s.) solution: Suppose that $\phi \in \mathrm{L}^{2}([0,1] \times \Omega)$ and $A, B \in$ $\mathrm{L}^{2}(\Omega)$. Then

$$
u_{t}(x, t)(\omega)=\alpha^{2}(\omega) u_{x x}(x, t)(\omega)
$$

a.s. for $x \in(0,1)$ and $t>0$, where the derivatives are understood in the classical sense; $u(0, t)(\omega)=A(\omega)$ and $u(1, t)(\omega)=B(\omega)$ a.s. for $t \geq 0$; and $u(x, 0)(\omega)=\phi(x)(\omega)$ a.s. for a.e. $x \in[0,1]$. Moreover, the process $u(x, t)(\omega)$ satisfying these conditions is unique.

ii) Mean square (m.s.) solution: Suppose that $\phi \in \mathrm{L}^{2}([0,1] \times \Omega), A, B \in$ $\mathrm{L}^{2}(\Omega)$ and $0<a \leq \alpha^{2}(\omega) \leq b$, a.e. $\omega \in \Omega$, for certain $a, b \in \mathbb{R}$. Then

$$
u_{t}(x, t)(\omega)=\alpha^{2}(\omega) u_{x x}(x, t)(\omega)
$$

a.s. for $x \in(0,1)$ and $t>0$, where the derivatives are understood in the m.s. sense; $u(0, t)(\omega)=A(\omega)$ and $u(1, t)(\omega)=B(\omega)$ a.s. for $t \geq 0$; 
and $u(x, 0)(\omega)=\phi(x)(\omega)$ a.s. for a.e. $x \in[0,1]$. Moreover, the process $u(x, t)(\omega)$ satisfying these conditions is unique.

In addition, under the assumptions of Proposition 7.2 , the solution to 7.1 is given by

$$
u(x, t)(\omega)=v(x, t)(\omega)+x B(\omega)+(1-x) A(\omega),
$$

where

$A_{k}(\omega)=2 \int_{0}^{1} \psi(y)(\omega) \sin (k \pi y) \mathrm{d} y, \quad \psi(y)(\omega)=\phi(y)(\omega)-y B(\omega)-(1-y) A(\omega)$.

$$
v(x, t)(\omega)=\sum_{k=1}^{\infty} A_{k}(\omega) \mathrm{e}^{-k^{2} \pi^{2} \alpha(\omega) t} \sin (k \pi x),
$$

The integral that defines $A_{k}(\omega)$ is understood in the sample path sense [125, Appendix A]. The convergence of the last series is considered a.s. or in $\mathrm{L}^{2}(\Omega)$, depending on whether we want $u(x, t)$ to be an a.s. or a m.s. solution, respectively.

As we are interested in computational uncertainty quantification, the existence of a solution in a probabilistic sense will not be a major concern. In contrast, our main goal is to construct reliable approximations of the probability density function to the solution stochastic process, $u(x, t)$, of the random heat diffusion problem (7.1). To achieve this goal, we take advantage of a random numerical scheme together with a key probabilistic result that will be introduced later, to construct a sequence of approximating density functions. Afterwards, the proposed method will be presented in an algorithm. We will present numerical experiments aimed to show the capability of the proposed to approach to quantify uncertainty in the random heat diffusion problem (7.1) via the computation of approximations to its probability density function.

\subsection{Method}

Consider the backward Euler method to formally approximate the solution process $u(x, t)$, [132]. We divide the spatial domain $[0,1]$ into equidistant points as $\left\{x_{0}, \ldots, x_{M+1}\right\}$, where $x_{i}=i h$ and $h=1 /(M+1)$. We discretize the time domain $[0, T]$ as $\left\{t_{0}, \ldots, t_{n}\right\}$, where $t_{n}=n k$ and $k=T / N$. The finite difference scheme corresponding to the backward Euler method is expressed via the following difference equation:

$$
u_{n+1, i}^{N, M}=u_{n, i}^{N, M}+\nu\left(u_{n+1, i+1}^{N, M}-2 u_{n+1, i}^{N, M}+u_{n+1, i-1}^{N, M}\right),
$$


where $\nu=\alpha k / h^{2}$ is a.s. positive. The term $u_{n, i}^{N, M}$ approximates $u\left(x_{i}, t_{n}\right)$. In matrix form,

$$
\mathbf{u}_{n+1}^{N, M}=\left(\mathbf{I}_{\mathbf{M}}-\nu \mathbf{L}\right)^{-1}\left(\mathbf{u}_{n}^{N, M}+\nu \mathbf{c}\right),
$$

where $\mathbf{u}_{n}^{N, M}=\left(u_{n, 1}^{N, M}, \ldots, u_{n, M}^{N, M}\right)^{\top}, \mathbf{c}=(A, 0, \ldots, 0, B)^{\top}(\top$ denotes the transpose operator for vectors and matrices), $\mathbf{I}_{\mathbf{M}}$ is the $M \times M$ identity matrix and

$$
\mathbf{L}=\left(\begin{array}{ccccc}
-2 & 1 & & & \\
1 & -2 & 1 & & \\
& \ddots & \ddots & \ddots & \\
& & 1 & -2 & 1 \\
& & & 1 & -2
\end{array}\right)
$$

is also an $M \times M$ matrix.

This numerical method is stable for all $\nu$. The random difference equation (7.5) approximates the partial differential equation (7.1) with order $\mathcal{O}(k)+\mathcal{O}\left(h^{2}\right)$. We will take $N=N_{M}=(M+1)^{2}$, so that the local truncation error is given by $\mathcal{O}\left(h^{2}\right)$. Therefore, we may drop the superscript $N$ from the random difference equation 7.5 :

$$
\mathbf{u}_{n+1}^{M}=\left(\mathbf{I}_{\mathbf{M}}-\nu \mathbf{L}\right)^{-1}\left(\mathbf{u}_{n}^{M}+\nu \mathbf{c}\right) .
$$

This linear recurrence $(7.6)$ has an explicit solution:

$$
\mathbf{u}_{n}^{M}=\mathcal{A}^{n} \phi^{M}+\left(\sum_{k=0}^{n-1} \mathcal{A}^{k}\right) \mathbf{b},
$$

where $\boldsymbol{\phi}^{M}=\left(\phi\left(x_{1}\right), \ldots, \phi\left(x_{M}\right)\right)^{\top}, \boldsymbol{A}=\left(\mathbf{I}_{\mathbf{M}}-\nu \mathbf{L}\right)^{-1}$ and $\mathbf{b}=\nu\left(\mathbf{I}_{\mathbf{M}}-\nu \mathbf{L}\right)^{-1} \mathbf{c}$, and from (7.7), we can compute explicitly each component $u_{n, i}^{M}$ :

$$
\begin{aligned}
u_{n, i}^{M} & =\left(\mathcal{A}^{n} \phi^{M}\right)_{i}+\left(\sum_{k=0}^{n-1} \mathcal{A}^{k}\right)(i,:) \mathbf{b} \\
& =\left(\mathcal{A}^{n} \phi^{M}\right)_{i}+\nu\left(\sum_{k=0}^{n-1} \mathcal{A}^{k}\right)(i,:) \mathcal{A}(:, 1) A+\nu\left(\sum_{k=0}^{n-1} \mathcal{A}^{k}\right)(i,:) \mathcal{A}(:, M) B
\end{aligned}
$$

where $\mathcal{A}(i,:)$ denotes the $i$-th row of $\mathcal{A}$ and $\mathcal{A}(:, j)$ refers to the $j$-th column of $\mathcal{A}$.

From (7.8), we will derive the probability distribution of $u_{n, i}^{M}$ on account of the distributions of $A, B$ and $\phi^{M}$. We will distinguish two scenarios: 
- Case 1: $A$ is an absolutely continuous random variable which is independent of the random vector $\left(\alpha, B, \phi^{M}\right)$.

- Case 2: $B$ is absolutely continuous and is independent of $\left(\alpha, A, \phi^{M}\right)$.

Since the analysis of Case 2 is analogous to the one of Case 1, we will detail the study corresponding to Case 1, and for the Case 2 we summarize the main conclusions.

The following lemma allows computing the probability density function of $u_{n, i}^{M}$. The result is an extension of the Random Variable Transformation technique (which has been extensively used [46, 52, 72]) when the transformation mapping consists of sums and products. Regarding notation, the probability density function of a random variable/vector $X$ will be denoted as $f_{X}$ hereafter, and its probability law will be written as $\mathbb{P}_{X}=\mathbb{P} \circ X^{-1}$.

Lemma 7.3 Let $U$ be an absolutely continuous random variable, independent of the random vector $\left(Z_{1}, Z_{2}\right)$, where $Z_{1} \neq 0$ a.s. Then $Z_{1} U+Z_{2}$ is absolutely continuous, with density function $f_{Z_{1} U+Z_{2}}(z)=\mathbb{E}\left[f_{U}\left(\left(z-Z_{2}\right) / Z_{1}\right) /\left|Z_{1}\right|\right]$.

Proof. Let $\mathcal{C}$ be a Borel set in $\mathbb{R}$. Then

$$
\begin{aligned}
& \mathbb{P}\left(Z_{1} U+Z_{2} \in \mathcal{C}\right)=\int_{\mathbb{R}^{2}} \mathbb{P}\left(Z_{1} U+Z_{2} \in \mathcal{C} \mid Z_{1}=z_{1}, Z_{2}=z_{2}\right) \mathbb{P}_{\left(Z_{1}, Z_{2}\right)}\left(\mathrm{d} z_{1}, \mathrm{~d} z_{2}\right) \\
& =\int_{\mathbb{R}^{2}} \mathbb{P}\left(z_{1} U+z_{2} \in \mathcal{C}\right) \mathbb{P}_{\left(Z_{1}, Z_{2}\right)}\left(\mathrm{d} z_{1}, \mathrm{~d} z_{2}\right)=\int_{\mathbb{R}^{2}} \int_{\left(\mathcal{C}-z_{2}\right) / z_{1}} f_{U}(u) \mathrm{d} u \mathbb{P}_{\left(Z_{1}, Z_{2}\right)}\left(\mathrm{d} z_{1}, \mathrm{~d} z_{2}\right) \\
& =\int_{\mathbb{R}^{2}} \int_{\mathcal{C}} f_{U}\left(\frac{u-z_{2}}{z_{1}}\right) \frac{1}{\left|z_{1}\right|} \mathrm{d} u \mathbb{P}_{\left(Z_{1}, Z_{2}\right)}\left(\mathrm{d} z_{1}, \mathrm{~d} z_{2}\right) \\
& =\int_{\mathcal{C}} \int_{\mathbb{R}^{2}} f_{U}\left(\frac{u-z_{2}}{z_{1}}\right) \frac{1}{\left|z_{1}\right|} \mathbb{P}_{\left(Z_{1}, Z_{2}\right)}\left(\mathrm{d} z_{1}, \mathrm{~d} z_{2}\right) \mathrm{d} u=\int_{\mathcal{C}} \mathbb{E}\left[f_{U}\left(\frac{u-z_{2}}{z_{1}}\right) \frac{1}{\left|z_{1}\right|}\right] \mathrm{d} u .
\end{aligned}
$$

By using this key lemma, we are able to compute, for the Case 1, the probability density function of $u_{n, i}^{M}$ by taking into account the representation given in (7.8). Take

$$
\begin{aligned}
& U=A \\
& Z_{1}=\nu\left(\sum_{k=0}^{n-1} \mathcal{A}^{k}\right)(i,:) \mathcal{A}(:, 1) \\
& Z_{2}=\left(\mathcal{A}^{n} \phi^{M}\right)_{i}+\nu\left(\sum_{k=0}^{n-1} \mathcal{A}^{k}\right)(i,:) \mathcal{A}(:, M) B .
\end{aligned}
$$


Let us justify that $Z_{1} \neq 0$ a.s. The matrix $\mathbf{I}_{\mathbf{M}}-\nu \mathbf{L}$ is an M-matrix, in the sense of ref. [99, p.10]: $\mathbf{I}_{\mathbf{M}}-\nu \mathbf{L}$ has its offdiagonal entries nonpositive and, for the vector $\mathbf{r}=\left(\sin (\pi j /(M+1))_{j=1}^{M}\right.$ with positive components, the image $\left(\mathbf{I}_{\mathbf{M}}-\nu \mathbf{L}\right) \mathbf{r}$ is written as $\lambda \mathbf{r}$, where $\lambda>0$, so that $\left(\mathbf{I}_{\mathbf{M}}-\nu \mathbf{L}\right) \mathbf{r}$ has positive components. Moreover, $\mathbf{I}_{\mathbf{M}}-\nu \mathbf{L}$ is an irreducible matrix, because its entries on the superdiagonal and on the subdiagonal are nonzero. By Theorem 2.7 in ref. [5], the entries of $\mathcal{A}=\left(\mathbf{I}_{M}-\nu \mathbf{L}\right)^{-1}$ are positive. Thus, $Z_{1}>0$.

The assumption of independence between $A$ and $\left(\alpha, B, \phi^{M}\right)$ implies the independence between $U$ and $\left(Z_{1}, Z_{2}\right)$, by [65, p. 93]. By applying Lemma 7.3, we obtain

$$
\begin{aligned}
& f_{u_{n, i}^{M}}(u)= \\
& \mathbb{E}\left[f_{A}\left(\frac{u-\left(\mathcal{A}^{n} \phi^{M}\right)_{i}-\nu\left(\sum_{k=0}^{n-1} \mathcal{A}^{k}\right)(i,:) \mathcal{A}(:, M) B}{\nu\left(\sum_{k=0}^{n-1} \mathcal{A}^{k}\right)(i,:) \mathcal{A}(:, 1)}\right) \frac{1}{\nu\left(\sum_{k=0}^{n-1} \mathcal{A}^{k}\right)(i,:) \mathcal{A}(:, 1)}\right] .
\end{aligned}
$$

For the Case 2, that is, if $B$ is absolutely continuous and is independent of $\left(\alpha, A, \phi^{M}\right)$, proceeding analogously we derive an alternative density function for $u_{n, i}^{M}$ :

$$
\begin{aligned}
& f_{u_{n, i}^{M}}(u)= \\
& \mathbb{E}\left[f_{B}\left(\frac{u-\left(\mathcal{A}^{n} \phi^{M}\right)_{i}-\nu\left(\sum_{k=0}^{n-1} \mathcal{A}^{k}\right)(i,:) \mathcal{A}(:, 1) A}{\nu\left(\sum_{k=0}^{n-1} \mathcal{A}^{k}\right)(i,:) \mathcal{A}(:, M)}\right) \frac{1}{\nu\left(\sum_{k=0}^{n-1} \mathcal{A}^{k}\right)(i,:) \mathcal{A}(:, M)}\right] .
\end{aligned}
$$

We approximate the density function of $u(x, t)$ as follows. Let $x_{0} \in(0,1)$ and $t_{0} \in(0, T)$. Consider a sequence of points in both partitions, $\left\{i_{M} /(M+1)\right\}_{M=1}^{\infty}$ and $\left\{n_{M} T / N_{M}\right\}_{M=1}^{\infty}$, where $i_{M} \in\{1, \ldots, M\}, n_{M} \in\left\{0, \ldots, N_{M}\right\}$ and $N_{M}=$ $(M+1)^{2}$, such that $i_{M} /(M+1) \rightarrow x_{0}$ and $n_{M} T / N_{M} \rightarrow t_{0}$ as $M \rightarrow \infty$. For example, take $i_{M}=\left\lfloor x_{0}(M+1)\right\rfloor$ and $n_{M}=\left\lfloor t_{0} N_{M} / T\right\rfloor$, where $\lfloor\cdot\rfloor$ stands for the integer part. Then, the density function of $u\left(x_{0}, t_{0}\right)$ is

$$
f_{u\left(x_{0}, t_{0}\right)}(u)=\lim _{M \rightarrow \infty} f_{u_{n_{M}, i_{M}}^{M}}(u) .
$$

At this point, we would like to remark that other finite difference schemes could be possible: forward Euler method, Crank-Nicholson method, etc. Our choice for the backward Euler method is due to its simplicity and being stable for all $\nu$. 
The case in which $A$ and $B$ are not absolutely continuous remains pending. One could think of performing the same analysis as before but isolating $\phi\left(x_{j}\right)$ in (7.8), instead of $A$ or $B$. In such a case, one assumes that $\phi\left(x_{j}\right)$ is absolutely continuous and independent of $\left(\alpha, A, B, \phi\left(x_{1}\right), \ldots, \phi\left(x_{j-1}\right), \phi\left(x_{j+1}\right), \ldots, \phi\left(x_{M}\right)\right)$. To achieve this independence, one may require $\phi\left(y_{1}\right), \ldots \phi\left(y_{m}\right)$ to be independent, for every $y_{1}, \ldots, y_{m} \in[0,1], m \geq 1$. A process $\phi$ of this type exists by Kolmogorov's Extension Theorem [8, Th. 36.2, p. 486]. However, by [92, Example 1.2.5, p. 10], this process $\phi$ is not jointly measurable on $[0,1] \times \Omega$, so that $\phi$ cannot belong to $\mathrm{L}^{2}([0,1] \times \Omega)$. Hence, Proposition 7.2 on the existence of solution does not apply.

\subsection{Computational aspects and algorithm}

In this section we comment on computational aspects regarding the implementation of the probability density function (7.9).

The computation of the powers $\mathcal{A}^{k}$ is especially demanding. Thus, it is better to consider the spectral decomposition of $\mathcal{A}$. The set of eigenvalues, $\mu_{l}$, and eigenvectors, $\mathbf{s}^{l}$, of $\mathcal{A}$ is well-known [123]:

$$
\mu_{l}=\frac{1}{2 \nu(1-\cos (l \pi h))+1}, \quad \mathbf{s}^{l}=(\sin (l \pi j h))_{j=1}^{M},
$$

for $l=1, \ldots, M$. Let $\mathbf{D}$ be the diagonal matrix with $\mathbf{D}_{l l}=\mu_{l}$, and let $\mathbf{P}=\left[\mathbf{s}^{1} \ldots \mathbf{s}^{M}\right]$ be the matrix whose column vectors are $\mathbf{s}^{1}, \ldots, \mathbf{s}^{M}$. Define $\mathbf{R}=\sqrt{2 /(M+1)} \mathbf{P}$. Then $\mathbf{R}$ is an orthogonal $M \times M$ matrix, and the decomposition $\mathcal{A}=\mathbf{R D R}{ }^{\top}$ holds. Hence, the powers of $\mathcal{A}$ can be computed as $\mathcal{A}^{k}=\mathbf{R D}^{k} \mathbf{R}^{\top}$, which reduces notably the computational load.

On the other hand, the theoretical expression of the expectation from (7.9) is the following:

$$
\begin{aligned}
f_{u_{n, i}^{M}}(u) & =\int_{\mathbb{R}^{M+2}} f_{A}\left(\frac{u-\left(\mathcal{A}^{n} \phi^{M}\right)_{i}-\nu\left(\sum_{k=0}^{n-1} \mathcal{A}^{k}\right)(i,:) \mathcal{A}(:, M) B}{\nu\left(\sum_{k=0}^{n-1} \mathcal{A}^{k}\right)(i,:) \mathcal{A}(:, 1)}\right) \\
& \times \frac{1}{\nu\left(\sum_{k=0}^{n-1} \mathcal{A}^{k}\right)(i,:) \mathcal{A}(:, 1)} \mathbb{P}_{\left(\alpha, B, \phi^{M}\right)}\left(\mathrm{d} \alpha, \mathrm{d} B, \mathrm{~d} \phi^{M}\right) .
\end{aligned}
$$

However, from a practical point of view, it is better to turn to Monte Carlo simulation to address the computation of 7.9 , 60]. Let $\alpha_{(j)}, B_{(j)}$ and $\phi_{(j)}^{M}$, 
$j=1, \ldots, m$, be $m$ realizations of the random variables/vectors $\alpha, B$ and $\phi^{M}$. Then we approximate $(7.9)$ as

$$
\begin{aligned}
f_{u_{n, i}^{M}}(u) & \approx \frac{1}{m} \sum_{j=1}^{m} f_{A}\left(\frac{u-\left(\mathcal{A}_{(j)}^{n} \phi_{(j)}^{M}\right)_{i}-\nu_{(j)}\left(\sum_{k=0}^{n-1} \mathcal{A}_{(j)}^{k}\right)(i,:) \mathcal{A}_{(j)}(:, M) B}{\nu_{(j)}\left(\sum_{k=0}^{n-1} \mathcal{A}_{(j)}^{k}\right)(i,:) \mathcal{A}_{(j)}(:, 1)}\right) \\
& \times \frac{1}{\nu_{(j)}\left(\sum_{k=0}^{n-1} \mathcal{A}_{(j)}^{k}\right)(i,:) \mathcal{A}_{(j)}(:, 1)} .
\end{aligned}
$$

When $m \rightarrow \infty$, this approximation becomes an a.s. limit by the Law of Large Numbers.

Based on this proposed computational method, we provide Algorithm 1 for uncertainty quantification for the solution stochastic process $u(x, t)$ of the random heat partial differential equation (7.1).

Once we obtain $F(u)$ in Algorithm 1, we can perform uncertainty quantification with the density function $F(u)$, because $F(u) \approx f_{u\left(x_{0}, t_{0}\right)}(u)$. For example, the expectation and variance of $u\left(x_{0}, t_{0}\right)$ may be approximated as

$$
\mathbb{E}\left[u\left(x_{0}, t_{0}\right)\right] \approx \int_{\mathbb{R}} u F(u) \mathrm{d} u, \quad \mathbb{V}\left[u\left(x_{0}, t_{0}\right)\right] \approx \int_{\mathbb{R}} u^{2} F(u) \mathrm{d} u-\left(\int_{\mathbb{R}} u F(u) \mathrm{d} u\right)^{2} .
$$

In the next section, we will illustrate the use of the proposed Algorithm 1 with two examples.

\subsection{Numerical examples}

Here, we show two numerical examples for 7.1 by considering a variety of probability distributions for each random input. Thus, we will demostrate the capability of the proposed approach to quantify uncertainty in the random diffusion model (7.1). Take $T=3$, so that the domain of the partial differential equation becomes $(0,1) \times(0,3)$, and let $u(x, t)$ be the solution stochastic process to (7.1), whose existence is guaranteed by [16, Th. 3.2]. We will approximate its probability density function at a point $\left(x_{0}, t_{0}\right)$ by utilizing (7.10). Consider $i_{M}=\left\lfloor x_{0}(M+1)\right\rfloor, n_{M}=\left\lfloor t_{0} N_{M} / T\right\rfloor$. These points satisfy $i_{M} /(M+1) \rightarrow x_{0}$ in the partitions, and $n_{M} T / N_{M} \rightarrow t_{0}$ as $M \rightarrow \infty$. 


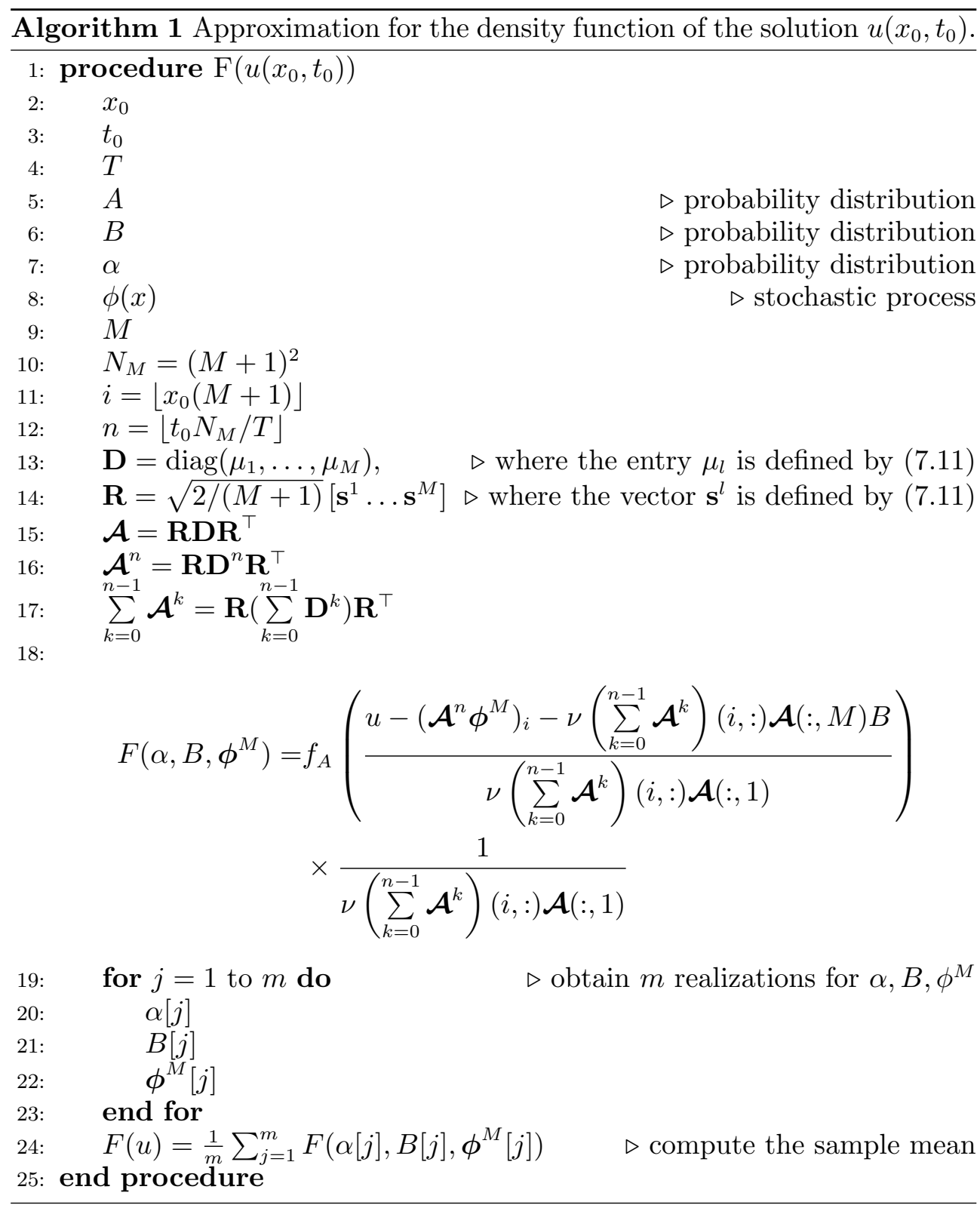


We will set particular distributions for $\alpha, A, B$ and $\phi$. It will be assumed that $A$ is absolutely continuous and independent of $(\alpha, B, \phi)$, so that Lemma 7.3 is applicable. The expectation 7.9 will be approximated via Monte Carlo simulations, with $m=20,000$ realizations of each random variable. This $m$ value has been checked to be enough in order to ensure a good approximation of (7.9).

In both examples, we observe fast convergence of $(7.9)$ as $M$ increases, although the error may not be monotonically decreasing.

To validate our results, we compare our computations of the expectation and variance (7.13) to the method proposed in ref. [47]. Suppose that $\alpha, A, B$ and $\phi$ are independent. The idea in ref. [47] is as follows: from $7.2-(7.3)-(7.4)$, the expectation of $u(x, t)$ may be approximated as

$$
\mathbb{E}[u(x, t)]=\mathbb{E}[v(x, t)]+x \mathbb{E}[B]+(1-x) \mathbb{E}[A],
$$

being

$$
\mathbb{E}[v(x, t)] \approx \sum_{k=1}^{K} \mathbb{E}\left[A_{k}\right] \mathbb{E}\left[\mathrm{e}^{-k^{2} \pi^{2} \alpha t}\right] \sin (k \pi x)
$$

and

$$
\mathbb{E}\left[A_{k}\right]=2 \int_{0}^{1} \mathbb{E}[\psi(y)] \sin (k \pi y) \mathrm{d} y, \quad \mathbb{E}[\psi(y)]=\mathbb{E}[\phi(y)]-y \mathbb{E}[B]-(1-y) \mathbb{E}[A],
$$

where the order of truncation $K$ must be sufficiently large to achieve the desired accuracy. Notice that the independence hypothesis between $\alpha$ and $\phi$ has been used in (7.15). Concerning the variance of $u(x, t)$, we compute

$$
\begin{gathered}
\mathbb{V}[u(x, t)]=\mathbb{V}[v(x, t)]+x^{2} \mathbb{V}[B]+(1-x)^{2} \mathbb{V}[A] \\
\mathbb{V}[v(x, t)] \approx \sum_{k_{1}, k_{2}=1}^{K} \mathbb{C o v}\left[A_{k_{1}}, A_{k_{2}}\right] \mathbb{E}\left[\mathrm{e}^{-\left(k_{1}^{2}+k_{2}^{2}\right) \pi^{2} \alpha t}\right] \sin \left(k_{1} \pi x\right) \sin \left(k_{2} \pi x\right) \\
+\sum_{k_{1}, k_{2}=1}^{K} \mathbb{E}\left[A_{k_{1}}\right] \mathbb{E}\left[A_{k_{2}}\right] \operatorname{Cov}\left[\mathrm{e}^{-k_{1}^{2} \pi^{2} \alpha t}, \mathrm{e}^{-k_{2}^{2} \pi^{2} \alpha t}\right] \sin \left(k_{1} \pi x\right) \sin \left(k_{2} \pi x\right),
\end{gathered}
$$

$$
\operatorname{Cov}\left[A_{k_{1}}, A_{k_{2}}\right]=4 \int_{0}^{1} \int_{0}^{1} \operatorname{Cov}\left[\psi\left(y_{1}\right), \psi\left(y_{2}\right)\right] \sin \left(k_{1} \pi y_{1}\right) \sin \left(k_{2} \pi y_{2}\right) \mathrm{d} y_{1} \mathrm{~d} y_{2},
$$




$$
\operatorname{Cov}\left[\psi\left(y_{1}\right), \psi\left(y_{2}\right)\right]=\operatorname{Cov}\left[\phi\left(y_{1}\right), \phi\left(y_{2}\right)\right]+y_{1} y_{2} \mathbb{V}[B]+\left(1-y_{1}\right)\left(1-y_{2}\right) \mathbb{V}[A], \quad(7.20)
$$

for a large order of truncation $K$. The independence assumptions have been used in (7.17), (7.18) and (7.20). The approximations obtained with these expressions from [47] must be similar to the those calculated with our density functions.

Example 7.4 Let $A \sim \operatorname{Gamma}(3,1), B=-1, \alpha \sim$ Triangular(1,2) and $\phi(x)=D \cos x+\mathrm{e}^{\sin \left(E x^{2}\right)}$, where $D \sim \operatorname{Binomial}(20,0.2), E \sim \operatorname{Uniform}(-1,0)$. By Proposition 7.2 , there exists a unique solution stochastic process $u(x, t)$, both in the a.s. and m.s. senses, which is given by $77.2-7.3-(7.4)$. It is assumed that $A, B, \alpha, D$ and $E$ are independent random variables. Let $\left(x_{0}, t_{0}\right)=(0.25,2.4)$. In Figure 7.1, we plot the approximations 7.9 for $M=$ $9,11,13,15,17,19,21$. We observe non-monotone convergence. Moreover, the density function obtained for $M=19$ coincides visually with the density function of $\lim _{t \rightarrow \infty} u(x, t)=x B+(1-x) A=-0.25+0.75 A$, which is given by

$$
f_{A}\left(\frac{x+0.25}{0.75}\right) \frac{1}{0.75} \text {. }
$$

This is because of $\lim _{t \rightarrow \infty} u(x, t)=x B+(1-x) A$ holds exponentially in $t$, so for $t=2.4$ we have $u(0.25,2.4) \approx u(0.25, \infty)=-0.25+0.75 A$.

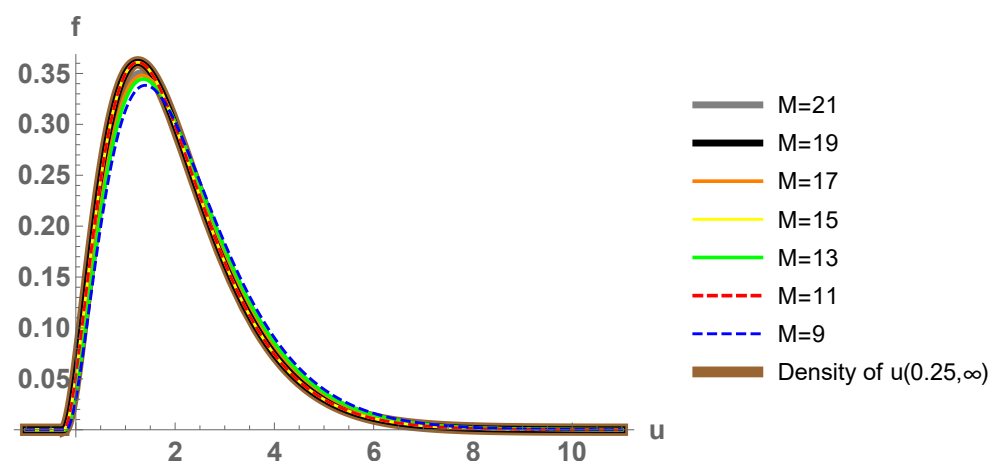

Figure 7.1: Example 7.4 plot of $f_{u_{n_{M}, i_{M}}^{M}}(u)$ given by 7.9 , and the asymptotic density 7.21 .

Table 7.1 shows the approximations for $\mathbb{E}\left[u\left(x_{0}, t_{0}\right)\right]$ and $\mathbb{V}\left[u\left(x_{0}, t_{0}\right)\right]$. Table 7.2 presents these computations by means of the procedure in ref. [47] (see 7.14)(7.20)). The agreement of the results demonstrates the validity of the probability density functions plotted in Figure 7.1. Moreover, notice that the results 
coincide with the asymptotic limit $\mathbb{E}[u(0.25, \infty)]=\mathbb{E}[-0.25+0.75 A]=2$ and $\mathbb{V}[u(0.25, \infty)]=\mathbb{V}[-0.25+0.75 A]=1.6875$.

\begin{tabular}{|c|c|c|c|c|c|c|c|}
\hline$M$ & 9 & 11 & 13 & 15 & 17 & 19 & 21 \\
\hline $\mathbb{E}\left[u_{n_{M}, i_{M}}^{M}\right]$ & 2.199 & 2 & 2.142 & 2 & 2.110 & 2 & 2.090 \\
\hline $\mathbb{V}\left[u_{n_{M}, i_{M}}^{M}\right]$ & 1.912 & 1.688 & 1.845 & 1.688 & 1.809 & 1.688 & 1.786 \\
\hline
\end{tabular}

Table 7.1: Example 7.4 approximations for $\mathbb{E}\left[u\left(x_{0}, t_{0}\right)\right]$ and $\mathbb{V}\left[u\left(x_{0}, t_{0}\right)\right]$ obtained with (7.13.

\begin{tabular}{|c|c|c|c|c|c|}
\hline$K$ & 1 & 2 & 3 & 4 & 5 \\
\hline $\mathbb{E}\left[u\left(x_{0}, t_{0}\right)\right]$ & 2 & 2 & 2 & 2 & 2 \\
\hline $\mathbb{V}\left[u\left(x_{0}, t_{0}\right)\right]$ & 1.688 & 1.688 & 1.688 & 1.688 & 1.688 \\
\hline
\end{tabular}

Table 7.2: Example 7.4 approximations for $\mathbb{E}\left[u\left(x_{0}, t_{0}\right)\right]$ and $\mathbb{V}\left[u\left(x_{0}, t_{0}\right)\right]$ as in ref. [47] (see (7.14)-(7.20).

Thus, the best choice for uncertainty quantification for $u(0.25,2.4)$ is $M=19$, or even the density function of $u(0.25, \infty)$ given by 7.21$)$.

Example 7.5 Let us consider $\alpha \sim$ Triangular(1,2), $A \sim \operatorname{Normal}(-1,1), B=$ -1 and

$$
\phi(x)=\sum_{j=1}^{\infty} \frac{\sqrt{2}}{j^{3 / 2} \sqrt{1+\log j}} \sin (j \pi x) \xi_{j},
$$

where $\xi_{1}, \xi_{2}, \ldots$ are independent and identically distributed random variables, with density function

$$
f_{\xi_{1}}(\xi)=\frac{\sqrt{2}}{\pi\left(1+\xi^{4}\right)}, \quad \xi \in \mathbb{R} .
$$

Since $\xi_{1}, \xi_{2}, \ldots$ have zero expectation and unit variance, and $\{\sqrt{2} \sin (j \pi x)\}_{j=1}^{\infty}$ is an orthonormal basis of $\mathrm{L}^{2}([0,1])$, the series in 7.22 corresponds to a Karhunen-Loève expansion [89, Th. 5.28]. The convergence of the series is understood in $\mathrm{L}^{2}([0,1] \times \Omega)$. Proposition 7.2 tells us that there is a unique solution stochastic process $u(x, t)$, both in the a.s. and m.s. senses, which is given by $7.2-7.3-7.4$.

In order to sample from $\phi(x)$, we need to truncate the series in 7.22 . Truncating up to order $N=5$, we are accounting for more than $99.5 \%$ of the total 
variance of $\phi(x), \int_{0}^{1} \mathbb{V}[\phi(x)] \mathrm{d} x=\sum_{j=1}^{\infty} 1 /\left(j^{3}(1+\log j)\right)$, [89, p. 204]. Thus, we will use

$$
\phi(x)=\sum_{j=1}^{5} \frac{\sqrt{2}}{j^{3 / 2} \sqrt{1+\log j}} \sin (j \pi x) \xi_{j},
$$

It is assumed that $A, B, \alpha, \xi_{1}, \ldots, \xi_{5}$ are independent random variables. Let $\left(x_{0}, t_{0}\right)=(0.25,0.3)$. Figure 7.2 shows the approximations 7.9 for values of $M=9,11,13,15,17,19,21,23$. Convergence is achieved, so that the density function of $u\left(x_{0}, t_{0}\right)$ has been accurately approximated.

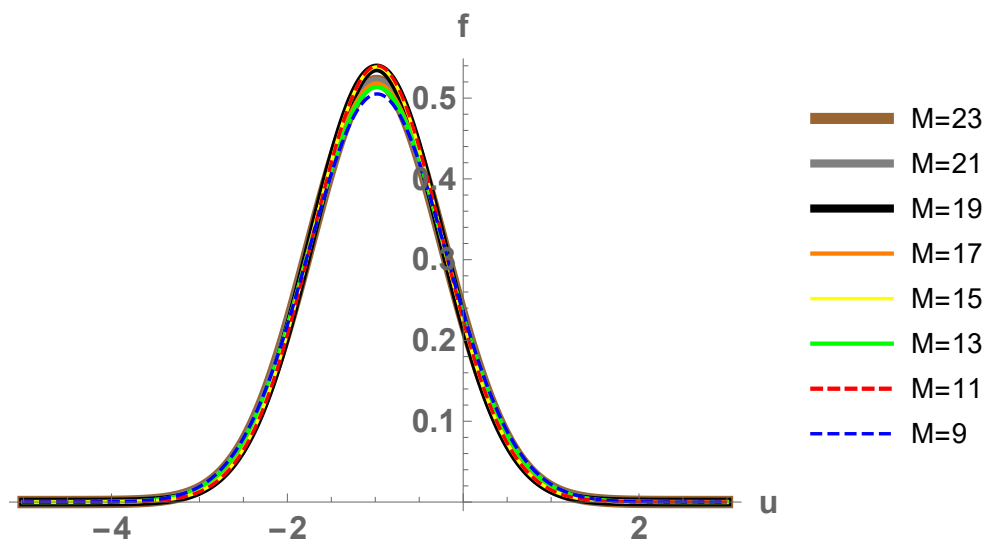

Figure 7.2: Example 7.5 plot of $f_{u_{n_{M}, i}^{M}}(u)$ given by 7.9 .

Table 7.3 shows the approximations for $\mathbb{E}\left[u\left(x_{0}, t_{0}\right)\right]$ and $\mathbb{V}\left[u\left(x_{0}, t_{0}\right)\right]$ obtained with (7.13). Notice that the convergence is non-monotone. We compare these estimates to the procedure in ref. [47] (see (7.14)-(7.20)), whose values are shown in Table 7.4. Again, the agreement of the results demonstrates the validity of the probability density functions plotted in Figure 7.2 .

\begin{tabular}{|c|c|c|c|c|c|c|c|c|}
\hline$M$ & 9 & 11 & 13 & 15 & 17 & 19 & 21 & 23 \\
\hline $\mathbb{E}\left[u_{n_{M}, i_{M}}^{M}\right]$ & -0.979 & -0.977 & -0.981 & -0.981 & -0.984 & -0.984 & -0.986 & -0.985 \\
\hline $\mathbb{V}\left[u_{n_{M}, i_{M}}^{M}\right]$ & 0.624 & 0.546 & 0.603 & 0.549 & 0.599 & 0.551 & 0.586 & 0.552 \\
\hline
\end{tabular}

Table 7.3: Example 7.5 approximations for $\mathbb{E}\left[u\left(x_{0}, t_{0}\right)\right]$ and $\mathbb{V}\left[u\left(x_{0}, t_{0}\right)\right]$ obtained with 7.13.

Thereby, the best truncation order for uncertainty quantification for $u(0.25,0.3)$ is $M=23$. 


\begin{tabular}{|c|c|c|c|c|c|}
\hline$K$ & 1 & 2 & 3 & 4 & 5 \\
\hline $\mathbb{E}\left[u\left(x_{0}, t_{0}\right)\right]$ & -0.987 & -0.987 & -0.987 & -0.987 & -0.987 \\
\hline $\mathbb{V}\left[u\left(x_{0}, t_{0}\right)\right]$ & 0.563 & 0.563 & 0.563 & 0.563 & 0.563 \\
\hline
\end{tabular}

Table 7.4: Example 7.5. approximations for $\mathbb{E}\left[u\left(x_{0}, t_{0}\right)\right]$ and $\mathbb{V}\left[u\left(x_{0}, t_{0}\right)\right]$ as in ref. 47] (see (7.14)-(7.20)

\subsection{Conclusions}

In this chapter, we have proposed a computational method to quantify the uncertainty of the random heat partial differential equation on a bounded domain via the approximation of its probability density function. The method is based on constructing a sequence of approximating density functions via a finite difference scheme. The numerical examples show that the convergence is achieved quickly, although with non-monotone decreasing error. Our approach improves the published contributions on heat transfer stochastic modeling, in which the approximation of the expectation and variance was the main goal. Moreover, our method could be applied to other relevant random ordinary and partial differential equations, in which the computation of the probability density function is a major goal.

\section{Acknowledgements}

This work has been supported by the Spanish Ministerio de Economía y Competitividad grant MTM2017-89664-P.

The main results of this chapter have been published in [15]. This chapter is a national collaboration with the researcher José Antonio Díaz Navas (Departamento de Óptica, Facultad de Ciencias, Universidad de Granada, Spain). 
Chapter 8

\section{Improvement of random differential models of growth of anaerobic photosynthetic bacteria by combining Bayesian inference and gPC}

The time evolution of microorganisms, such as bacteria, is of great interest in biology. In the article by D. Stanescu et al. [Electronic Transactions on Numerical Analysis, 34, 44-58 (2009)], a logistic model was proposed to model the growth of anaerobic photosynthetic bacteria. In the laboratory experiment, actual data for two species of bacteria were considered: $R$. capsulatus and C. vibrioforme. In this chapter, we suggest a new nonlinear model by assuming that the population growth rate is not proportional to the size of the bacteria population, but to the number of interactions between the microorganisms, and by taking into account the beginning of the death phase in the kinetic curve. Stanescu et al. evaluated the effect of randomness into the model coefficients by using generalized Polynomial Chaos ( $g P C)$ expansions, by setting arbitrary distributions without taking into account the likelihood of the data. By contrast, we utilize a Bayesian inverse approach for parameter estimation to obtain reliable posterior distributions for the random input coefficients in both the logistic and our new model. Since our new model does not possess an explicit solution, we use $g P C$ expansions to construct the Bayesian model and to accelerate the Markov Chain Monte Carlo algorithm for the Bayesian inference. 


\subsection{Introduction}

The time evolution of microorganisms, such as bacteria, has been of great interest in biology for decades [56, 101, 105]. In this regard, mathematical models are important to understand and generalize laboratory experiments and to make predictions [61, 69, 79, 87, 93, 102, 148. These models are usually continuous systems that involve ordinary or partial differential equations, which depend on input parameters (initial conditions, forcing term and/or coefficients, etc.) often with a biological interpretation (carrying capacity, growth rate, birth or death rate, concentration of nutrients, etc.). If experimental values are available for the model coefficients, we have a forward model to describe and forecast the main features of the biological system. But in general, to determine the model parameters, experimental data needs to be used. The process of adjusting the coefficients in virtue of collected data is called an inverse problem.

Deterministic differential equations have been widely studied from a theoretical and numerical point of view. To solve the inverse problem in this setting, one usually turns to optimization algorithms, for instance a least squares fitting.

However, deterministic models do not take into account the inherent uncertainty associated to biological processes. Inaccuracies in the measurements often arise due to errors in the laboratory experiments (human, mechanical, etc.), lack of information, missed data, etc. It thus becomes necessary to treat the input parameters in a random sense. This gives rise to random ordinary and partial differential equations [106, 125, 129].

The primary objective when dealing with random models is uncertainty quantification, i.e., understanding the main statistical features of the forward random model predictions. There are different techniques in the existing literature to handle stochastic systems. When a closed form solution of the random model is not available, one of the best and computationally cheapest methods for uncertainty quantification is the gPC technique: the solution stochastic process is expressed as a mean square limit of Galerkin projections onto subspaces of orthogonal polynomials [142, 143].

To solve an inverse problem in a random setting, gPC by itself cannot determine suitable model coefficients. The Bayesian approach allows making statistical inference from prior probabilistic information of the parameters and the likelihood associated to the data. The output of Bayesian inference is a 
posterior probability distribution for each of the parameters, which permits quantifying uncertainty via the posterior predictive distribution [38, 86, 100].

When the solution of the random differential equation does not have a closed form expression, each sampling point of the Markov Chain Monte Carlo algorithm requires a numerical solution of the differential equation, which might be time consuming. The gPC and Galerkin projection techniques give approximations of the solution stochastic process, which may be used in the Bayesian model to accelerate the numerical simulation [96, 97, 142].

In this chapter, we apply this technique to model the growth of anaerobic photosynthetic bacteria. The population of bacteria increases in size by using light energy to reduce $\mathrm{CO}_{2}$. In the laboratory experiment, two species of bacteria were considered: Rhodobacter capsulatus ( $R$. capsulatus) and Chlorobium vibrioforme ( $C$. vibrioforme). Direct cell counts were made every two or three days until a stationary phase was achieved. This measurements give two sets of data for each one of the two populations under study. In [127], the authors considered a logistic model to explain bacterial growth in both populations, based on Malthusian exponential growth model (first-order kinetics equation) and competitiveness when there is scarcity of nutrients (mainly light and $\mathrm{CO}_{2}$ ). We suggest a new nonlinear model equation by assuming that the population growth rate is not proportional to the size of the bacteria population, but to the number of interactions between the microorganisms (the squared abundance), and by taking into account the start of the decline phase. In [127], uncertainty is put into the model by using arbitrary distributions for the coefficients. By contrast, we have utilized a Bayesian inverse approach for parameter estimation. Since our model does not have an explicit solution, we have combined gPC expansions together with the stochastic Galerkin projection technique to accelerate the Bayesian inference. Thus, we assess the effect of randomness and quantify the uncertainty in a rigorous way.

The structure of the chapter is the following. In Section 8.2, we show and explain the experimental data, and we analyze empirically which should be a good differential equation model. In Section 8.3, we provide a comprehensive analysis of the logistic model and show its associated Bayesian model. In Section 8.4. we expose the main theoretical features of the combination of $\mathrm{gPC}$ expansions and Bayesian inference. We extend its applicability to models with random variance of the errors, and we use the logistic model as a test example. In Section 8.5, we expose the theoretical ideas to improve the modeling from [127. Section 8.6 is devoted to numerical experiments for deterministic fittings and uncertainty quantification. Finally, Section 8.7 draws the conclusions. 


\subsection{Data on anaerobic photosynthetic bacterial growth}

We will use experimental data from the growth of anaerobic photosynthetic bacteria under infrared lightning conditions. The population of bacteria increases in size by using light energy to reduce $\mathrm{CO}_{2}$ (photosynthesis). In the laboratory experiment, two species of bacteria were considered: Rhodobacter capsulatus ( $R$. capsulatus) and Chlorobium vibrioforme ( $C$. vibrioforme). For further details about the experiment, we refer the reader to [127. Table 8.1] shows laboratory data on the population sizes of $R$. capsulatus and $C$. vibrioforme under infrared lightning conditions in different mediums. The number of cells $/ \mathrm{mL}$ has been rescaled by dividing by $10^{6}$. Figure 8.1 plots the cell counts from Table 8.1 .

\begin{tabular}{cc|cc}
$\begin{array}{c}\text { Time } \\
\text { (days) }\end{array}$ & $\begin{array}{c}\text { R. capsulatus } \\
\text { Population }\end{array}$ & $\begin{array}{c}\text { Time } \\
\text { (cells/mL, scale } 10^{6} \text { ) }\end{array}$ & $\begin{array}{c}\text { vibrioforme } \\
\text { (days) }\end{array}$ \\
\begin{tabular}{cc|c} 
(cells/mL, scale $10^{6}$ ) \\
\hline 0
\end{tabular} & 0.583 & 0 & 0.986 \\
2 & 0.635 & 14 & 2.41 \\
4 & 1.08 & 16 & 2.24 \\
7 & 3.20 & 18 & 4.21 \\
9 & 5.23 & 21 & 5.72 \\
11 & 5.28 & 23 & 5.99 \\
14 & 5.30 & 25 & 7.86 \\
& & 28 & 6.52
\end{tabular}

Table 8.1: Bacteria population sizes [127.

At the first days, when there is no competition between bacteria and no limitation of resources (light and $\mathrm{CO}_{2}$ ), the population seems to increase with exponential growth. This was the model proposed by Thomas Malthus in 1798 in his essay [95]. A more modern formulation of the Malthusian growth model can be read at the introductory text [102]. The importance of the Malthusian model is evident as in the field of population ecology it is considered as the first law of population dynamics [133]. In his written essay, Malthus already described how non-abundance of sustenance would affect the growth of species. This contribution was developed by Verhulst in 1838 [136]: as time passes and the number of microorganisms augments, there is more competition for the limited food, so that the growth rate decreases with the size of the population. This fact is the basis of the so-called logistic model. Its modern formulation can be consulted in [102], for instance. In principle, the logistic model corresponds to the observed s-shape in Figure 8.1. This 
$R$ capsulatus

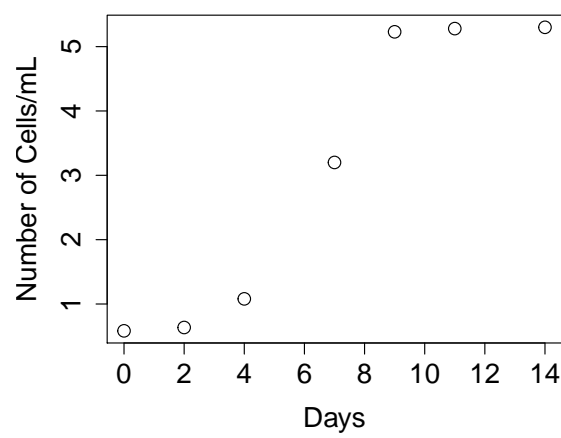

C vibrioforme

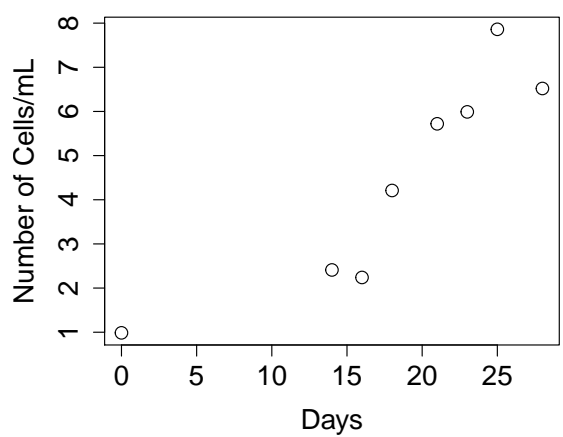

Figure 8.1: Population size of $R$. capsulatus (first plot) and C. vibrioforme (second plot).

applies especially for $R$. capsulatus. By contrast, $C$. vibrioforme presents a drop of the amount of bacteria at the end, so the logistic model may not fit as expected. This descent might come from the commence of the death phase.

We will see that, in both groups of $R$. capsulatus and $C$. vibrioforme, better results are obtained if we consider that, for the first days, the rate of change is not proportional to the population size, but to the total number of interactions between the microorganisms, i.e., to the squared abundance. Also, the introduction of the death rate in the modeling will play a key role. Taking into account the competition for the limited resources as time goes on, we will obtain a variation of the logistic model that will allow a better modeling for the data from Figure 8.1. This sort of model formulation has not been widely used in the biological modeling literature, and the unique reference on utilizing squared abundance for the modeling of the growth rate that we have found has been [112, pp. 17-18]. Its justification from a dynamics standpoint is not as clear as the exponential or logistic growth models. However, in our particular database and from a mathematical point of view, this idea of employing squared abundance works better than the logistic model. 


\subsection{Logistic model}

Let $y(t)$ be the total population at time $t$ measured in days. If there is no competition or there is sustenance for life, and the rate of change is proportional to the total abundance, the Malthusian exponential model describes the population growth [95, 102]: $y^{\prime}(t)=r y(t)$. Given an initial condition $y\left(t_{0}\right)=y_{0}$, the unique solution of this model is given by $y(t)=y_{0} \mathrm{e}^{r t}$. In Figure 8.1, this model seems suitable for the first 9 days in the $R$. capsulatus group and for the first 21 days in the $C$. vibrioforme population. However, at the 9 th and 21st days, respectively, an inflection point in the bacterial growth is observed, due to the limited abundance of resources (light and $\mathrm{CO}_{2}$ ). If we take into account this scarcity of substances as time passes, then we obtain the logistic model [102, 136]:

$$
y^{\prime}(t)=r y(t)\left(1-\frac{y(t)}{K}\right) .
$$

The term $K$ is the carrying capacity. In the logistic equation, it is assumed that the growth rate lags linearly with the population size. Under the initial condition $y\left(t_{0}\right)=y_{0}$, the ordinary differential equation (8.1) has a unique solution:

$$
y(t)=\frac{y_{0} K}{y_{0}+\left(K-y_{0}\right) \mathrm{e}^{-r t}} .
$$

In the following subsections, we will review the fit of model $(8.1)-(8.2)$ to the data from Table 8.1 done in 127. Later, the coefficients in (8.1) will be randomized. In [127], a gPC approach was used to evaluate the effect of randomness in the coefficients. Distributions for the coefficients were set by the authors just from an empirical point of view, without using the information given by the data, i.e., the likelihood. By contrast, we will determine appropriate posterior distributions for the input coefficients of (8.1), by utilizing Bayesian inference.

\subsubsection{Deterministic curve fitting}

Given the data from Table 8.1, the authors in [127] calculated deterministic coefficients $r, y_{0}$ and $K$ in (8.2) such that the squared error is minimized. This method is usually called least squares fitting. Given a set of collected data $d_{1}, \ldots, d_{N}$ at times $t_{1}, \ldots, t_{N}$ and given the output of a model $M(\zeta, t)$, where $\zeta$ are the input parameters and $t$ is the time, the squared error is expressed as

$$
\sum_{i=1}^{N}\left(d_{i}-M\left(\zeta, t_{i}\right)\right)^{2} .
$$


A least squares fitting consists in finding the set of parameters $\zeta_{0}$ such that

$$
\min _{\zeta} \sum_{i=1}^{N}\left(d_{i}-M\left(\zeta, t_{i}\right)\right)^{2}=\sum_{i=1}^{N}\left(d_{i}-M\left(\zeta_{0}, t_{i}\right)\right)^{2} .
$$

This last expression $(8.3)$ is called residual squared error. The best model should minimize the residual squared error. In Table 8.2 , the estimates for the coefficients and the residual squared error are shown. In Figure 8.2, we plot the least squares fitting together with the measured data. These computations have been already done in [127. We observe that the deterministic logistic model approximates well the data, although the last data from $C$. vibrioforme presents problems due to its unexpected decreasing behavior.

Parameters for $R$. capsulatus Parameters for C. vibrioforme

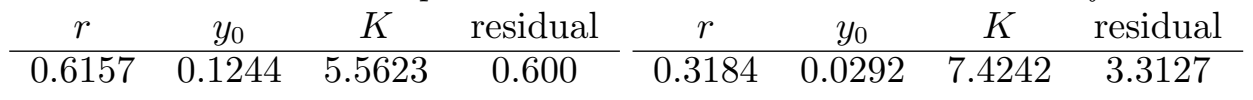

Table 8.2: Parameters for $R$. capsulatus and $C$. vibrioforme under the logistic model.
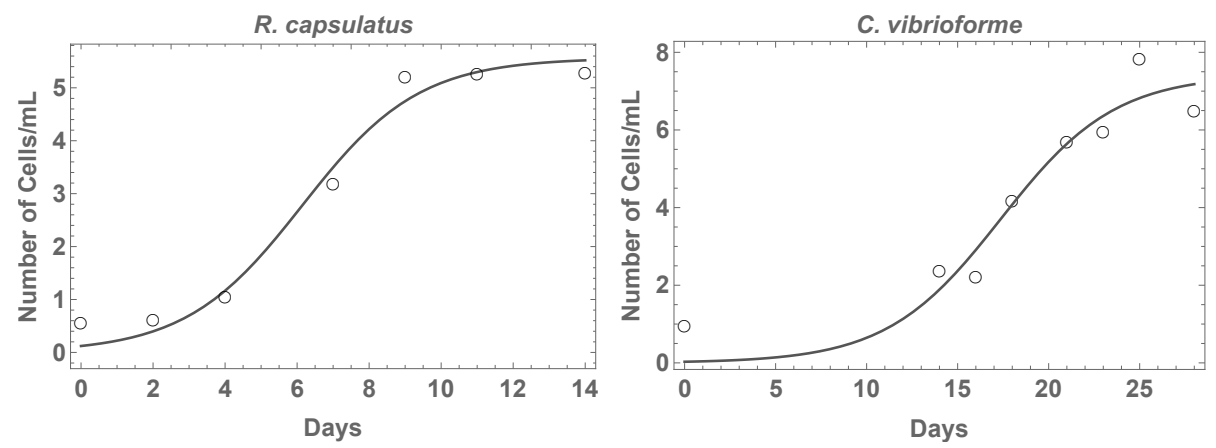

Figure 8.2: Least squares fitting of $R$. capsulatus (first plot) and $C$. vibrioforme (second plot) under the logistic model. The real data is denoted by $\circ$ and the fitting is given by the black continuous line. 


\subsubsection{Random coefficients and Bayesian inference}

Due to the variability involved in the measurements (errors in the collection of data, lack of information, etc.) and the inherent uncertainty associated to population dynamics phenomena, it would be better to treat the input coefficients in a random sense. That is, we suppose that the parameters $r, y_{0}$ and $K$ from the logistic model (8.1) depend on an experiment $\omega: r=r(\omega)$, $y_{0}=y_{0}(\omega)$ and $K=K(\omega)$. We denote by $\Omega$ the set of all experiments $\omega$, equipped with a $\sigma$-algebra $\mathcal{F}$ and a probability measure $\mathbb{P}$, so that we have an underlying probability space $(\Omega, \mathcal{F}, \mathbb{P})[142$, Def. 2.4]. In this context, the solution $y(t)$ given by 8.2 becomes a stochastic process $y(t, \omega)$. The main objective thus becomes to quantify the uncertainty of $y(t, \omega)$, for example, with the computation of its mean and variance. When no analytical expression can be obtained, a computational approach needs to be used.

In [127], some probability distributions are given to $r, y_{0}$ and $K$, to assess the effect of randomness into the input parameters. The distributions are set empirically, without utilizing the information given by the data, that is to say, the likelihood. Thus, in order to improve the methodology from [127, we propose a Bayesian model to determine reliable (posterior) distributions for $r, y_{0}$ and $K$. Let $\left(t_{1}, \ldots, t_{N}\right)$ be the times of interest: for $R$. capsulatus they are $\left(t_{1}, t_{2}, t_{3}, t_{4}, t_{5}, t_{6}, t_{7}\right)=(0,2,4,7,9,11,14)$, and for $C$. vibrioforme they are $\left(t_{1}, t_{2}, t_{3}, t_{4}, t_{5}, t_{6}, t_{7}\right)=(0,14,16,18,21,23,25,28)$, respectively (see Table 8.1). Let $y_{i}$ be the random variable that models the size of the population at time $t_{i}$. The Bayesian model takes the following form:

$$
\begin{gathered}
\left(y_{1}, \ldots, y_{N}\right) \mid\left(r, y_{0}, K, \sigma\right) \sim \pi\left(y_{1}, \ldots, y_{N} \mid r, y_{0}, K, \sigma\right)=\prod_{i=1}^{N} \pi\left(y_{i} \mid r, y_{0}, K, \sigma\right) \\
\pi\left(r, y_{0}, K, \sigma\right)=\pi(r) \pi\left(y_{0}\right) \pi(K) \pi(\sigma) \\
r \sim \pi(r), \quad y_{0} \sim \pi\left(y_{0}\right), \quad K \sim \pi(K), \quad \sigma \sim \pi(\sigma) .
\end{gathered}
$$

Here, $\pi$ denotes the corresponding probability density function. In 8.4 , it is assumed that the errors caused by the logistic model for $t_{1}, \ldots, t_{N}$ are independent random variables, with zero expectation and variance $\sigma^{2}$. In fact, bearing in mind expression 8.2 for the solution of the logistic equation, we will set

$$
\pi\left(y_{i} \mid r, y_{0}, K, \sigma\right) \sim \operatorname{Normal}\left(\frac{y_{0} K}{y_{0}+\left(K-y_{0}\right) \mathrm{e}^{-r t_{i}}}, \sigma\right),
$$

i.e., the errors are taken Gaussian. In 8.5, we reflect the fact that the parameters are independent a priori. We have set this prior independence because we do not have any prior information about the covariances, and the forthcoming 
gPC theory will be exposed for independent random inputs. When computing the joint posterior distribution, the values of the posterior covariances will adapt to the data, so there will possibly be no independence. Finally, in 8.6 , we set the prior distributions for $r, y_{0}, K$ and $\sigma$.

The distributions for $y_{0}$ and $K$ must be positive, as they measure bacteria abundance. The distribution for $\sigma$ is also positive by definition of variance. Concerning $r$, looking at Figure 8.1 we deduce that $r$ should be positive as well. Nonetheless, in practice, it could be possible to set prior probability distributions with support intersecting negative numbers, provided that we put a positive mean value and a very small variance.

On the other hand, the distributions of $r, y_{0}$ and $K$ should have as mean value the deterministic estimates from Table 8.2. From an intuitive point of view, these deterministic estimates are the unique information available to set the prior distributions $\pi(r), \pi\left(y_{0}\right)$ and $\pi(K)$. While from a mathematical standpoint, we know that, when the sample size $N$ is large, the posterior distribution follows approximately a normal law with mean value given by the maximum likelihood estimator (combine [85, Th. 3.10] for the asymptotic behavior of the maximum likelihood estimator and [85, Th. 8.3] for the asymptotic limit of the Bayesian estimator). In this case, as the errors are supposed to be Gaussian and independent, the maximum likelihood estimator coincides with the least squares fitting performed in Table 8.2. Notice that, taking the deterministic estimates from Table 8.2 as the mean values for the prior distributions, we are employing the collected data to set the priors, so that we are actually using the so-called empirical Bayes method [86, Ch. 9], [31, 25].

The formula for the joint posterior density function of the parameters is given by

$$
\pi\left(r, y_{0}, K, \sigma \mid y_{1}, \ldots, y_{N}\right)=\frac{\pi\left(y_{1}, \ldots, y_{N} \mid r, y_{0}, K, \sigma\right) \pi\left(r, y_{0}, K, \sigma\right)}{\iiint \int \pi\left(y_{1}, \ldots, y_{N} \mid r, y_{0}, K, \sigma\right) \pi\left(r, y_{0}, K, \sigma\right) \mathrm{d} r \mathrm{~d} y_{0} \mathrm{~d} K \mathrm{~d} \sigma} .
$$

To compute the posterior density function of a subset of the random vector $\left(r, y_{0}, K, \sigma\right)$, just marginalize by integrating. The posterior predictive distribution is expressed as

$\pi\left(\tilde{y}_{1}, \ldots, \tilde{y}_{N} \mid y_{1}, \ldots, y_{N}\right)=\iiint \int \pi\left(\tilde{y}_{1}, \ldots, \tilde{y}_{N} \mid r, y_{0}, K, \sigma\right) \pi\left(r, y_{0}, K, \sigma \mid y_{1}, \ldots, y_{N}\right) \mathrm{d} r \mathrm{~d} y_{0} \mathrm{~d} K \mathrm{~d} \sigma$.

These formulas and more information on Bayesian inference are available in the standard reference book [86]. Usually, the posterior distribution of the model parameters is not analytically tractable, therefore simulation algorithms must be carried out to sample from the posterior distribution. This class of algorithms is encompassed under the name Markov Chain Monte Carlo 
simulation [86, Ch. 6-7], 131]. An algorithm for Bayesian simulation has been implemented in WinBUGS [86, Ch. 8], 91]. Other possible software are JAGS [86, p. 214], [50, 78, 108]; SAS (SAS Institute, Cary NC) [86, Ch. 8], [128; etc.

In Section 8.6, we will specify prior distributions for the parameters $r, y_{0}$ and $K$. We will thus assess the effect of randomness into the inputs of the random logistic model.

\subsection{Combining Bayesian inference and gPC}

In this section we will show how gPC expansions can be used to perform Bayesian inference when the solution of the differential equation model does not have an explicit expression. There are results in the extant literature that combine Bayesian inference and gPC expansions when the error from the model is assumed to be Gaussian, with zero expectation and constant variance [96, 97], [142, Ch. 8]. We will extend these results to a random variance, so that one does not have to make point estimate guesses on the variability of the model error. We will show how these theoretical results work with the random logistic model. This will be a test example, since we saw in the previous section that the logistic equation has a closed form solution, to which Bayesian inference can be directly applied. By contrast, in Section 8.5, in which we improve the logistic equation to a more suitable model, we will need gPC expansions to carry out accelerated Bayesian inference, since the solution of the new model equation will not be explicitly known.

\subsubsection{Theoretical results}

Consider an ordinary differential equation model $y^{\prime}(t)=F(t, y(t))$. Suppose that both $F$ and the solution $y(t)$ depend on some random input parameters $\zeta_{1}, \ldots, \zeta_{s}$ (the initial condition is among these inputs). The probabilistic properties of these random inputs are: mutual independence, absolute continuity and finiteness of all moments. Let $\zeta=\left(\zeta_{1}, \ldots, \zeta_{s}\right)$ be the joint vector of coefficients. Suppose that we have times of interest, $t_{1}, \ldots, t_{N}$, in which we have collected data $d_{1}, \ldots, d_{N}$. We suppose that $y^{\prime}(t)=F(t, y(t))$ is the suitable deterministic model to explain $d_{1}, \ldots, d_{N}$, and from it we construct the following Bayesian model: if we denote by $y_{i}$ the random variable that 
models $d_{i}$, then

$$
\begin{gathered}
\left(y_{1}, \ldots, y_{N}\right) \mid \zeta \sim \prod_{i=1}^{N} \operatorname{Normal}\left(y\left(t_{i}\right), \sigma\right), \\
\zeta \sim \pi(\zeta)=\prod_{i=1}^{N} \pi\left(\zeta_{i}\right), \quad \sigma \sim \pi(\sigma) .
\end{gathered}
$$

We are assuming that $\sigma$ is either absolutely continuous with density function $\pi(\sigma)$ or a constant. In the case of being a constant, the subsequent development is applicable by considering a Dirac delta function as its density function. Let

$$
\pi\left(\zeta, \sigma \mid d_{1}, \ldots, d_{N}\right)=\frac{\pi\left(d_{1}, \ldots, d_{N} \mid \zeta, \sigma\right) \pi(\zeta, \sigma)}{\iint \pi\left(d_{1}, \ldots, d_{N} \mid \zeta, \sigma\right) \pi(\zeta, \sigma) \mathrm{d} \zeta \mathrm{d} \sigma}
$$

be the joint posterior density function of the parameters. This Bayesian model was proposed in the previous section for the random logistic model, and it makes sense when the explicit solution $y(t)$ of the differential equation model is available.

When $y(t)$ does not have a closed form expression and one has to use Markov Chain Monte Carlo algorithms [86, Ch. 6-7], 131], the main computational drawback is that each sampling point requires a solution of the underlying stochastic system $y^{\prime}(t)=F(t, y(t))$. The idea to speed up the Bayesian inference is to approximate $y(t)$ via another function in an $\mathrm{L}^{2}(\Omega)$ sense, and then to put the approximation in the mean of the normal distribution from (8.7).

To approximate $y(t)$, we use the gPC technique. We work in the Hilbert space $\left(\mathrm{L}^{2}(\Omega),\langle\rangle,\right)$ of random variables with finite variance, where the inner product is defined as $\langle X, Y\rangle=\mathbb{E}[X Y]$. Suppose that the random inputs $\zeta_{1}, \ldots, \zeta_{s}$ are independent and absolutely continuous random variables with finite moments and density functions $\pi\left(\zeta_{1}\right), \ldots, \pi\left(\zeta_{s}\right)$ (the prior density functions defined in (8.8)). There are different gPC approaches, which are kept to a minimum below:

(i) (Classical) gPC: Suppose that $\zeta_{1}, \ldots, \zeta_{s}$ are functions of random variables with distributions that belong to the Askey-Wiener scheme. That is, $\zeta=h(\xi)$, where $h: \mathbb{R}^{r} \rightarrow \mathbb{R}^{s}$ is a Borel measurable function and $\xi=\left(\xi_{1}, \ldots, \xi_{r}\right)$ is a random vector with independent components, such that $\xi_{i}$ is Gaussian, Gamma, Beta or uniform distributed. Take the univariate orthogonal polynomials from the Askey-Wiener scheme associated to the distribution of $\xi_{i}, 1 \leq i \leq r$, and compute a simple tensor product to obtain multivariate orthogonal polynomials in $\xi$. Let $\left\{\phi_{i}(\xi)\right\}_{i=1}^{\infty}$ be the sequence of orthogonal polynomials with respect to $\langle$,$\rangle . As y(t)$ is 
a function of $\xi$, we can expand $y(t)=\sum_{i=1}^{\infty} \tilde{y}_{i}(t) \phi_{i}(\xi)$ in $\mathrm{L}^{2}(\Omega)$, where $\tilde{y}_{i}(t)=\mathbb{E}\left[y(t) \phi_{i}(\xi)\right] / \mathbb{E}\left[\phi_{i}(\xi)^{2}\right]$ is the $i$-th Fourier coefficient. This approach is based on [142, 143].

(ii) Adaptive gPC: In this case, the distributions of $\zeta_{1}, \ldots, \zeta_{s}$ do not necessarily belong to the Askey-Wiener scheme. Let $\mathcal{C}_{i}^{p}=\left\{1, \zeta_{i}, \ldots, \zeta_{i}^{p}\right\}$ be the canonical basis of polynomials in $\zeta_{i}$ up to degree $p$. We orthonormalize this basis with respect to $\langle$,$\rangle and thus get \Xi_{i}^{p}=\left\{\phi_{0}^{i}\left(\zeta_{i}\right), \ldots, \phi_{p}^{i}\left(\zeta_{i}\right)\right\}$. Using a simple tensor product, we define an orthonormal basis with respect to $\langle$,$\rangle in the space of multivariate polynomials in \zeta$ up to degree $p: \Xi=\left\{\phi_{1}(\zeta), \ldots, \phi_{P}(\zeta)\right\}$, where $P=(p+s) ! / p ! s !$. If we let $p, P \rightarrow \infty$, we obtain an orthonormal sequence $\left\{\phi_{i}(\zeta)\right\}_{i=1}^{\infty}$. We expand $y(t)$ as $y(t)=\sum_{i=1}^{\infty} \tilde{y}_{i}(t) \phi_{i}(\zeta)$, where $\tilde{y}_{i}(t)=\mathbb{E}\left[y(t) \phi_{i}(\zeta)\right]$ is the $i$-th Fourier coefficient. This approach is based on [33, 46] and is referred to as adaptive gPC. An advantage of this strategy is that we are not restricted to standard probability distributions, because of the Random Variable Transformation technique 29]. However, using the Gram-Schmidt procedure may lead to a loss of orthogonality for large $p[62$, which may ruin the computations. Nonetheless, adaptive gPC usually converges in an algebraic or exponential rate (spectral convergence), so a small $p$ usually suffices and no loss of orthogonality problems appear.

For convergence issues on the (classical) gPC and adaptive gPC expansions, we refer the reader to [58, Th. 3.6], which completely determines the problem of convergence: (i) if $y(t) \in \mathrm{L}^{2}(\Omega)$, then $y(t)=\sum_{i=1}^{\infty} \tilde{y}_{i}(t) \phi_{i}(\xi)$ if the moment problem is uniquely solvable for each random variable $\xi_{1}, \ldots, \xi_{r}$; (ii) if $y(t) \in$ $\mathrm{L}^{2}(\Omega)$, then $y(t)=\sum_{i=1}^{\infty} \tilde{y}_{i}(t) \phi_{i}(\zeta)$ if the moment problem is uniquely solvable for each random variable $\zeta_{1}, \ldots, \zeta_{s}$.

We will adopt the adaptive gPC approach (ii), as it allows more general probability distributions for $\zeta_{1}, \ldots, \zeta_{s}$ for the stochastic Galerkin projection technique, without having to compute the inverse of their cumulative distribution functions, see [142, expr. (5.15) (5.16)], [127, expr. (4.8) (4.9)]. The stochastic Galerkin projection technique is based on approximating $y(t) \approx$ $\sum_{i=1}^{P} \hat{y}_{i}^{P}(t) \phi_{i}(\zeta)=\hat{y}^{P}(t)$, by imposing $\hat{y}^{P}(t)$ to be a solution of the differential equation. Using the orthonormality of $\phi_{1}(\zeta), \ldots, \phi_{P}(\zeta)$, we obtain a deterministic system of differential equations for the coefficients $\hat{y}_{1}^{P}(t), \ldots, \hat{y}_{P}^{P}(t)$ :

$$
\begin{gathered}
\frac{\mathrm{d}}{\mathrm{d} t} \hat{y}_{k}^{P}(t)=\left\langle F\left(t, \sum_{i=1}^{P} \hat{y}_{i}^{P}(t) \phi_{i}(\zeta)\right), \phi_{k}(\zeta)\right\rangle, \\
\hat{y}_{k}^{P}(0)=\mathbb{E}\left[y_{0} \phi_{k}(\zeta)\right],
\end{gathered}
$$


for $k=1, \ldots, P$. Under certain conditions, the Galerkin projection $\hat{y}^{P}(t)$ tends in $\mathrm{L}^{2}(\Omega)$ as $P \rightarrow \infty$ to $y(t)$, see [121].

Consider the Bayesian model

$$
\begin{gathered}
\left(y_{1}, \ldots, y_{N}\right) \mid \zeta \sim \prod_{i=1}^{N} \operatorname{Normal}\left(\hat{y}^{P}\left(t_{i}\right), \sigma\right), \\
\zeta \sim \pi(\zeta)=\prod_{i=1}^{N} \pi\left(\zeta_{i}\right), \quad \sigma \sim \pi(\sigma) .
\end{gathered}
$$

Let

$$
\pi_{P}\left(\zeta, \sigma \mid d_{1}, \ldots, d_{N}\right)=\frac{\pi_{P}\left(d_{1}, \ldots, d_{N} \mid \zeta, \sigma\right) \pi(\zeta, \sigma)}{\iint \pi_{P}\left(d_{1}, \ldots, d_{N} \mid \zeta, \sigma\right) \pi(\zeta, \sigma) \mathrm{d} \zeta \mathrm{d} \sigma}
$$

be the joint posterior density of the parameters, where $\pi_{P}\left(d_{1}, \ldots, d_{N} \mid \zeta, \sigma\right)$ is the likelihood from 8.9 and $\pi(\zeta, \sigma)$ is the prior from 8.10$)$ which coincides with 8.8). In [97, the authors proved that, if $\hat{y}^{P}(t) \rightarrow y(t)$ in $\mathrm{L}^{2}(\Omega)$ as $P \rightarrow \infty$ and $\sigma$ is constant, then $\pi_{P}\left(\zeta \mid d_{1}, \ldots, d_{N}\right)$ tends to $\pi\left(\zeta \mid d_{1}, \ldots, d_{N}\right)$ as $P \rightarrow \infty$ in the sense of the Kullback-Leibler divergence:

$$
D\left(\pi_{P} \| \pi\right)=\int \pi_{P}\left(\zeta \mid d_{1}, \ldots, d_{N}\right) \log \frac{\pi_{P}\left(\zeta \mid d_{1}, \ldots, d_{N}\right)}{\pi\left(\zeta \mid d_{1}, \ldots, d_{N}\right)} \mathrm{d} \zeta \stackrel{P \rightarrow \infty}{\longrightarrow} 0 .
$$

Moreover, if $\hat{y}^{P}(t) \rightarrow y(t)$ in $\mathrm{L}^{2}(\Omega)$ algebraically/exponentially, then we have $D\left(\pi_{P} \| \pi\right) \rightarrow 0$ algebraically/exponentially.

Let us see that this result can be extended to a random $\sigma$ that possesses a prior distribution $\pi(\sigma)$. In [97, Lemma 4.2, expr. (4.11)], we need to add an integration with respect to $\pi(\sigma)$. If we impose

$$
\mathbb{E}_{\pi(\sigma)}\left[\frac{1}{\sigma^{3 N}}\right]=\int_{0}^{\infty} \frac{\pi(\sigma)}{\sigma^{3 N}} \mathrm{~d} \sigma<\infty,
$$

then the same conclusion from [97, Lemma 4.2] holds:

$$
D\left(\pi_{P}|| \pi\right)=\iint \pi_{P}\left(\zeta, \sigma \mid d_{1}, \ldots, d_{N}\right) \log \frac{\pi_{P}\left(\zeta, \sigma \mid d_{1}, \ldots, d_{N}\right)}{\pi\left(\zeta, \sigma \mid d_{1}, \ldots, d_{N}\right)} \mathrm{d} \zeta \mathrm{d} \sigma \stackrel{P \rightarrow \infty}{\longrightarrow} 0 .
$$

Thus, under general assumptions, $\pi_{P}\left(\zeta, \sigma \mid d_{1}, \ldots, d_{N}\right) \rightarrow \pi\left(\zeta, \sigma \mid d_{1}, \ldots, d_{N}\right)$ in the sense of the Kullback-Leibler divergence. Formally, the posterior predictive distribution computed from the Galerkin projection $8.9-8.10$ tends to the posterior predictive distribution from $(8.7)-8.8$ :

$$
\pi_{P}\left(\tilde{d}_{1}, \ldots, \tilde{d}_{N} \mid d_{1}, \ldots, d_{N}\right)=\iint \pi_{P}\left(\tilde{d}_{1}, \ldots, \tilde{d}_{N} \mid \zeta, \sigma\right) \pi_{P}\left(\zeta, \sigma \mid d_{1}, \ldots, d_{N}\right) \mathrm{d} \zeta \mathrm{d} \sigma
$$


$\stackrel{P \rightarrow \infty}{\longrightarrow} \pi\left(\tilde{d}_{1}, \ldots, \tilde{d}_{N} \mid d_{1}, \ldots, d_{N}\right)=\iint \pi\left(\tilde{d}_{1}, \ldots, \tilde{d}_{N} \mid \zeta, \sigma\right) \pi\left(\zeta, \sigma \mid d_{1}, \ldots, d_{N}\right) \mathrm{d} \zeta \mathrm{d} \sigma$.

\subsubsection{Application to the random logistic model}

Consider the Bayesian model (8.4) - 8.6). Instead of using the solution (8.2) from the logistic differential equation (8.1), we approximate it via a stochastic Galerkin procedure as a test of the previous theory. Let $\zeta=\left(r, y_{0}, K\right)$ and $\hat{y}^{P}(t)=\sum_{i=1}^{P} \hat{y}_{i}^{P}(t) \phi_{i}(\zeta)$. The system of differential equations for the coefficients $\hat{y}_{1}^{P}(t), \ldots, \hat{y}_{P}^{P}(t)$ is

$$
\begin{gathered}
\frac{\mathrm{d}}{\mathrm{d} t} \hat{y}_{k}^{P}(t)=\sum_{i=1}^{P} \hat{y}_{i}^{P}(t) \mathbb{E}\left[r \phi_{i}(\zeta) \phi_{k}(\zeta)\right]-\sum_{i, j=1}^{P} \hat{y}_{i}^{P}(t) \hat{y}_{j}^{P}(t) \mathbb{E}\left[\frac{r}{K} \phi_{i}(\zeta) \phi_{j}(\zeta) \phi_{k}(\zeta)\right], \\
\hat{y}_{k}^{P}(0)=\mathbb{E}\left[y_{0} \phi_{k}(\zeta)\right], k=1, \ldots, P .
\end{gathered}
$$

Using standard numerical techniques, the Galerkin coefficients $\hat{y}_{1}^{P}(t), \ldots, \hat{y}_{P}^{P}(t)$ can be computed at the times of interest $t_{1}, \ldots, t_{N}$. To do statistical inference, we use the model 8.9)-8.10). In Section 8.6, we show numerical experiments. We will see that the posterior distribution $\pi_{P}\left(\zeta, \sigma \mid d_{1}, \ldots, d_{N}\right)$ is similar to the true posterior $\pi\left(\zeta, \sigma \mid d_{1}, \ldots, d_{N}\right)$ from (8.4)-(8.6), even for small $p$ and $P$, due to the spectral convergence.

\subsection{Improvement of the logistic model}

Suppose that, under non-scarcity of nourishment, the growth rate $y^{\prime}(t)$ is not proportional to the population size $y(t)$, but to the total number of interactions, i.e., to the squared abundance $y(t)^{2}$. In this case, the model becomes a variation of the Malthusian growth model: $y^{\prime}(t)=r y(t)^{2}$. If we take into account competition inside the test tubes because of limited resources, mainly light and $\mathrm{CO}_{2}$, then the growth rate constant decays linearly with the population size:

$$
y^{\prime}(t)=r y(t)^{2}\left(1-\frac{y(t)}{K}\right)
$$

The coefficient $K$ is the carrying capacity. Unlike the logistic differential equation, given an initial condition $y\left(t_{0}\right)=y_{0}$, the ordinary differential equation 8.11) does not have an explicit form for the solution.

This model formulation (8.11) has not been extensively used in the biological modeling literature, and the unique reference on squared abundance for the modeling of the growth rate that we have found has been [112, pp. 17-18]. 
Its biological justification is debatable, nonetheless, in our particular database and from a mathematical standpoint, (8.11) works better than the logistic model.

Model 8.11 may be improved if we take into account the death rate:

$$
y^{\prime}(t)=r y(t)^{2}\left(1-\frac{y(t)}{K}\right)-\delta y(t) .
$$

After the stationary phase, bacteria population enters into the so-called death phase. Due to the lack of nutrients, the population size starts to decline. In this model, $K$ is interpreted as the carrying capacity under no mortality.

At this point, we can compare models 8.1), 8.11) and 8.12 from the following mathematical point of view. Consider a general model $y^{\prime}(t)=f(y(t))$. If we assume that $f$ is sufficiently smooth, then we can express $f$ as a Taylor power series: $f(x)=a_{0}+a_{1} x+a_{2} x^{2}+a_{3} x^{3}+\ldots$. If we suppose that there is no spontaneous generation in the population, then $a_{0}=0$. Therefore, the model equation becomes $y^{\prime}(t)=a_{1} y(t)+a_{2} y(t)^{2}+a_{3} y(t)^{3}+\ldots$. If we truncate at the first term, then we obtain Malthus model. If we keep the first and second term, the logistic equation appears. If we put $a_{1}=0$ and keep $a_{2}$ and $a_{3}$, we get model (8.11). Finally, if we take the first three terms, the differential equation becomes (8.12). This provides an intuition on why 8.12 should make the most significant improvement. Indeed, we will see that model (8.12) improves the fitting of both the logistic equation 8.1 and model 8.11.

\subsubsection{Deterministic curve fitting}

Given the data from Table 8.1, we get deterministic estimates for $r, y_{0}, K$ and $\delta$ in 8.11 and 8.12 via a least squares procedure 8.3 . In this case, the least squares fitting has to be performed without an explicit solution of the differential equation model. In Section 8.6, we will show the least squares fitting and the residual squared error. We will observe that the error is much smaller for 8.11) and 8.12 than for the logistic model (8.1), especially for the $R$. capsulatus population. Therefore, from a deterministic point of view, taking into account interactions instead of total population allows a better modeling. The best modeling will be achieved with (8.12). This highlights the importance of adding the effect of mortality in the equation. 


\subsubsection{Random coefficients and combination of Bayesian inference and $g P C$}

To randomize both 8.11 and 8.12 , we consider that the output depends on an experiment $\omega$, which belongs to the sample space $\Omega$ of an underlying probability space $(\Omega, \mathcal{F}, \mathbb{P})$. Thereby, the parameters are random variables: $r=r(\omega), y_{0}=y_{0}(\omega), K=K(\omega), \delta=\delta(\omega)$; and the solution $y(t)$ becomes a stochastic process $y(t, \omega)$. The goal is to quantify its uncertainty computationally, by approximating its mean and variance statistics.

We model the bacterial growth with Bayesian model (8.7)-(8.8). As no explicit solution of (8.11) is available, we approximate $y(t)$ in $\mathrm{L}^{2}(\Omega)$ using the stochastic Galerkin projection technique. Let $\left\{\phi_{i}(\zeta)\right\}_{i=1}^{P}$ be the orthonormal sequence from the adaptive gPC approach, where $\zeta=\left(r, y_{0}, K\right)$. Let $\hat{y}^{P}(t)=\sum_{i=1}^{P} \hat{y}_{i}^{P}(t) \phi_{i}(\zeta)$ be the Galerkin projection. The deterministic coefficients are computed by solving numerically the following system of deterministic differential equations:

$$
\begin{aligned}
\frac{\mathrm{d}}{\mathrm{d} t} \hat{y}_{k}^{P}(t)= & \sum_{i, j=1}^{P} \hat{y}_{i}^{P}(t) \hat{y}_{j}^{P}(t) \mathbb{E}\left[r \phi_{i}(\zeta) \phi_{j}(\zeta) \phi_{k}(\zeta)\right] \\
- & \sum_{i, j, l=1}^{P} \hat{y}_{i}^{P}(t) \hat{y}_{j}^{P}(t) \hat{y}_{l}^{P}(t) \mathbb{E}\left[\frac{r}{K} \phi_{i}(\zeta) \phi_{j}(\zeta) \phi_{l}(\zeta) \phi_{k}(\zeta)\right], \\
& \hat{y}_{k}^{P}(0)=\mathbb{E}\left[y_{0} \phi_{k}(\zeta)\right], k=1, \ldots, P .
\end{aligned}
$$

In the case of model 8.12 , letting $\zeta=\left(r, y_{0}, K, \delta\right)$ and using a similar reasoning, we get the following system of deterministic differential equations:

$$
\begin{gathered}
\frac{\mathrm{d}}{\mathrm{d} t} \hat{y}_{k}^{P}(t)=\sum_{i, j=1}^{P} \hat{y}_{i}^{P}(t) \hat{y}_{j}^{P}(t) \mathbb{E}\left[r \phi_{i}(\zeta) \phi_{j}(\zeta) \phi_{k}(\zeta)\right] \\
-\sum_{i, j, l=1}^{P} \hat{y}_{i}^{P}(t) \hat{y}_{j}^{P}(t) \hat{y}_{l}^{P}(t) \mathbb{E}\left[\frac{r}{K} \phi_{i}(\zeta) \phi_{j}(\zeta) \phi_{l}(\zeta) \phi_{k}(\zeta)\right]-\sum_{i=1}^{P} \hat{y}_{i}^{P}(t) \mathbb{E}\left[\delta \phi_{i}(\zeta) \phi_{k}(\zeta)\right], \\
\hat{y}_{k}^{P}(0)=\mathbb{E}\left[y_{0} \phi_{k}(\zeta)\right], k=1, \ldots, P .
\end{gathered}
$$

Bayesian model 8.9 8 - 8.10 permits assessing the effect of randomness in (8.11) and (8.12) using the likelihood of the data.

We remark that the prior distributions for $r, y_{0}, K, \delta$ and $\sigma$ must be positive, although in practice it could be possible to set prior distributions taking 
negative values as long as we put a positive mean value and a very small variance.

In the numerical experiments, the prior distributions will be strongly centered around the deterministic value obtained in the least squares fitting. As we already explained, this is because for a large sample size $N$, the posterior distribution follows approximately a normal law with mean value given by the maximum likelihood estimator. In Section 8.6, we show the simulation results from WinBUGS.

\subsection{Numerical experiments}

In this section, we perform numerical experiments of the models presented before. First, we will specify prior distributions for $r, y_{0}$ and $K$ to carry out Bayesian inference in the logistic model 8.1). As a checking performance, we will see that expressing the solution 8.2 of the logistic equation 8.1 via gPC expansions gives good approximations of the posterior and posterior predictive distributions. On the other hand, we will fit the new models proposed, 8.11) and 8.12), in a deterministic manner to compare the results with those achieved in [127. Finally, we will combine Bayesian inference and gPC expansions to simulate from the posterior distributions of the random parameters with a cheap computational expense.

\subsubsection{Random logistic model}

Consider the logistic model 8.1 and its solution 8.2 in a randomized setting. To model the data from Table 8.1 using the Bayesian approach $8.4-8.6$, we have set

$$
r \sim \operatorname{Gamma}\left(\alpha_{r}, \beta_{r}\right), y_{0} \sim \operatorname{Gamma}\left(\alpha_{y_{0}}, \beta_{y_{0}}\right), K \sim \operatorname{Gamma}\left(\alpha_{K}, \beta_{K}\right), \sigma \sim \operatorname{Unif}\left(a_{\sigma}, b_{\sigma}\right)
$$

(recall that these four parameters are positive), where we have employed the shape-rate notation for the gamma distribution. As we want the mean of these random variables to be equal to the least square estimates (maximum likelihood estimators) from Table 8.2, we impose:

$$
\begin{aligned}
& \text { R. capsulatus: } \frac{\alpha_{r}}{\beta_{r}}=0.6157, \frac{\alpha_{y_{0}}}{\beta_{y_{0}}}=0.1244, \frac{\alpha_{K}}{\beta_{K}}=5.5623, \\
& \text { C. vibrioforme: } \frac{\alpha_{r}}{\beta_{r}}=0.3184, \frac{\alpha_{y_{0}}}{\beta_{y_{0}}}=0.0292, \frac{\alpha_{K}}{\beta_{K}}=7.4242 .
\end{aligned}
$$


For the $R$. capsulatus group, we suppose that approximately $68 \%$ of the variability of $r, y_{0}$ and $K$ is $0.05,0.05$ and 0.2 , respectively (this is the subjective part of the modeling), and we take this as the typical deviation (by the 68-9599.7 rule [109]). Then

$$
\text { R. capsulatus : } \frac{\alpha_{r}}{\beta_{r}^{2}}=0.0025, \frac{\alpha_{y_{0}}}{\beta_{y_{0}}^{2}}=0.0025, \frac{\alpha_{K}}{\beta_{K}^{2}}=0.04 \text {. }
$$

For the C. vibrioforme population, we take

$$
\text { C. vibrioforme: } \frac{\alpha_{r}}{\beta_{r}^{2}}=0.0009, \frac{\alpha_{y_{0}}}{\beta_{y_{0}}^{2}}=0.0009, \frac{\alpha_{K}}{\beta_{K}^{2}}=0.01 \text {. }
$$

This gives, for the $R$. capsulatus population, the values $\alpha_{r}=151.6346, \beta_{r}=$ $246.28, \alpha_{y_{0}}=6.190144, \beta_{y_{0}}=49.76, \alpha_{K}=773.4795$ and $\beta_{K}=139.0575$; and for the $C$. vibrioforme population, the parameters $\alpha_{r}=112.64, \beta_{r}=353.78$, $\alpha_{y_{0}}=0.947, \beta_{y_{0}}=32.44, \alpha_{K}=5511.87$ and $\beta_{K}=742.42$. For $\sigma$, assuming that the error in the modeling is at most 1 , we set $\sigma \sim \operatorname{Unif}(0,1)$.

With this information, we have the prior distributions for the parameters. The posterior distribution of the model parameters is not analytically tractable, so simulation algorithms must be carried out to sample from the posterior distribution (Markov Chain Monte Carlo simulation) [86, Ch. 6-7], [131]. The Bayesian model has been implemented in WinBUGS [86, Ch. 8], 91]. We fixed a burnin period of 75,000 iterations and simulated 150,000 samples of the parameters. We executed two chains with different initial values to assess convergence. The computer timing was 47 seconds for the burnin period, plus 94 seconds for the later 150,000 samples. In Table 8.3, we show a descriptive analysis of the posterior distributions. In Figure 8.3 and Figure 8.4, the posterior density function is plotted for each of the parameters. Figure 8.5 presents the means and credible intervals from the posterior predictive distributions at times $t_{1}, \ldots, t_{N}$. We observe that the means provide good estimations for the data. Moreover, the credible intervals contain all data points.

\subsubsection{Random logistic model and gPC expansions}

Consider the logistic model (8.1) and its randomized solution (8.2). We use Bayesian model (8.9) - 8.10) as an approximation of the Bayesian approach 8.4 - 8.6), with the same prior distributions as in (8.13), just as a test example of the theory exposed. As before, the posterior distribution of the model parameters is not analytically tractable, so we have used WinBUGS to simulate samples from the posterior distributions. We simulated 150,000 samples of the model parameters, after having removed the first 75,000 iterations (burnin 
Posterior distributions for $R$. capsulatus

\begin{tabular}{cccc} 
Parameter & Mean & sd & 0.95 interval \\
\hline$r$ & 0.619 & 0.039 & $(0.544,0.699)$ \\
$y_{0}$ & 0.126 & 0.034 & $(0.069,0.203)$ \\
$K$ & 5.560 & 0.164 & $(5.243,5.888)$
\end{tabular}

Posterior distributions for C. vibrioforme

\begin{tabular}{cccc} 
Parameter & Mean & sd & 0.95 interval \\
\hline$r$ & 0.321 & 0.025 & $(0.274,0.373)$ \\
$y_{0}$ & 0.031 & 0.015 & $(0.010,0.069)$ \\
$K$ & 7.421 & 0.098 & $(7.229,7.614)$
\end{tabular}

Table 8.3: Descriptive table for the posterior distributions of the parameters for $R$. capsulatus and C. vibrioforme under the random logistic model 8.1.
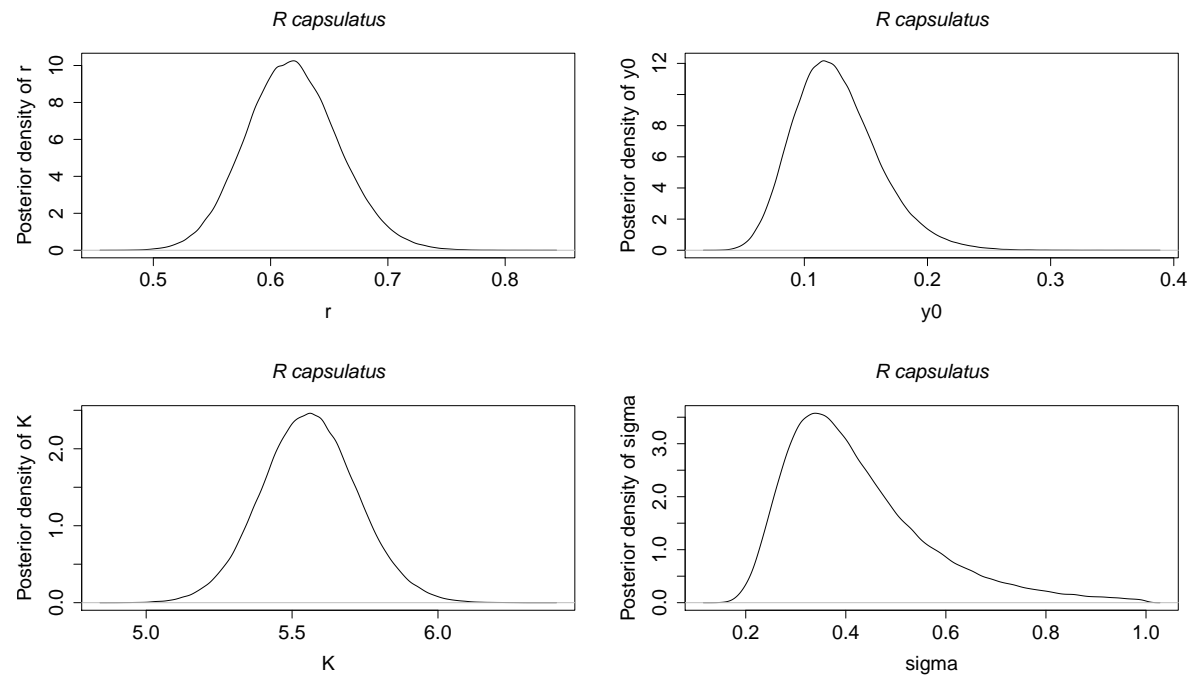

Figure 8.3: Posterior distributions for the model parameters of $R$. capsulatus under the random logistic model 8.1 . 

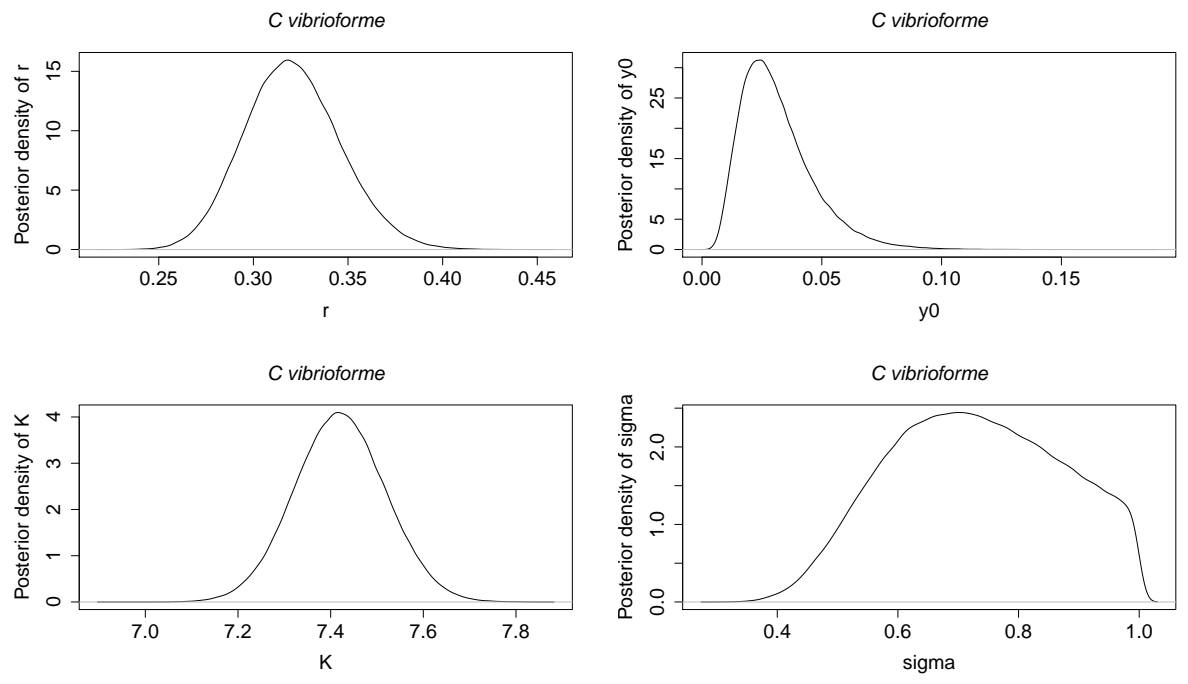

Figure 8.4: Posterior distributions for the model parameters of $C$. vibrioforme under the random logistic model (8.1).
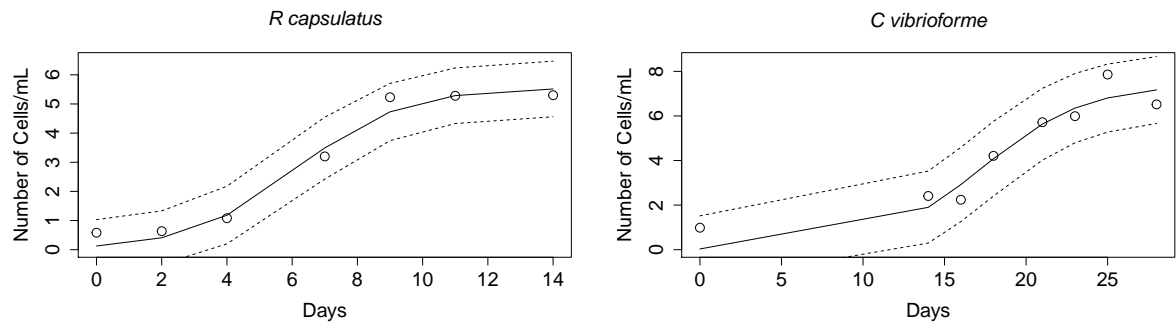

Figure 8.5: Model fitting for $R$. capsulatus (first plot) and C. vibrioforme (second plot) under the random logistic model (8.1). The real data is denoted by $\circ$, the fitting is given by the black continuous line and the 0.95 credible interval is drawn with dashed lines. 
period). We executed two chains with distinct initial iterates to evaluate convergence. The execution timing was 47 seconds for the burnin period, plus 94 seconds for the later 150,000 samples. In Table 8.4, Figure 8.6, Figure 8.7 and Figure 8.8, we show the results from the Bayesian inference with $p=2$. Observe that the estimates are similar to those from Table 8.3, Figure 8.3, Figure 8.4 and Figure 8.5, although $p=2$ is a small order of truncation. This is due to the spectral convergence of the stochastic Galerkin projection.

\begin{tabular}{|c|c|c|c|}
\hline \multicolumn{4}{|c|}{ Posterior distributions for $R$. capsulatus } \\
\hline Parameter & Mean & sd & 0.95 interval \\
\hline$r$ & 0.6215 & 0.04073 & $(0.5431,0.7028)$ \\
\hline$y_{0}$ & 0.1262 & 0.03524 & $(0.06632,0.2043)$ \\
\hline$K$ & 5.557 & 0.1652 & $(5.238,5.888)$ \\
\hline \multicolumn{4}{|c|}{ Posterior distributions for $C$. vibrioforme } \\
\hline Parameter & Mean & sd & 0.95 interval \\
\hline$r$ & 0.3338 & 0.02593 & $(0.2837,0.3842)$ \\
\hline$y_{0}$ & 0.02825 & 0.01403 & $(0.003839,0.05829)$ \\
\hline$K$ & 7.418 & 0.0989 & $(7.226,7.613)$ \\
\hline
\end{tabular}

Table 8.4: Descriptive table for the posterior distributions of the parameters for $R$. capsulatus and C. vibrioforme under the random logistic model 8.1 with gPC expansions.

\subsubsection{Improvement of the logistic model}

Consider the new model (8.11), in which the Malthusian growth rate is substituted by taking into account squared abundance. We perform a least squares fitting 8.3 to find the optimal estimates for $r, y_{0}$ and $K$. In Table 8.5, we show the estimates and the residual squared error. We observe that the error is much smaller for 8.11 than for the logistic model 8.1), especially for the $R$. capsulatus population. Figure 8.9 shows how accurate is the approximation with model (8.11). Hence, from a deterministic standpoint, taking into account interactions instead of total abundance allows a better modeling.

Concerning the new model 8.12 , in which we take into account the death rate, we also perform a least squares fitting 8.3 to get the optimal estimates for $r$, $y_{0}, K$ and $\delta$. Table 8.6 and Figure 8.10 present the results. The deterministic fitting improves that of model 8.11). 

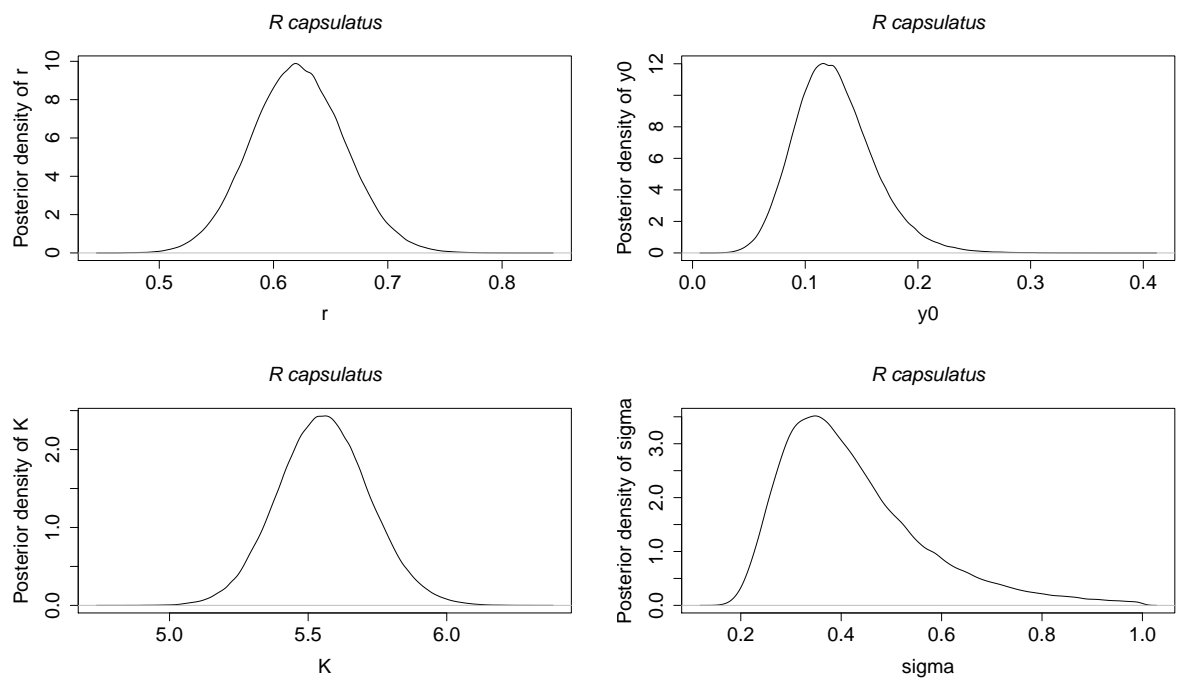

Figure 8.6: Posterior distributions for the model parameters of $R$. capsulatus under the random logistic model 8.1 with gPC expansions.
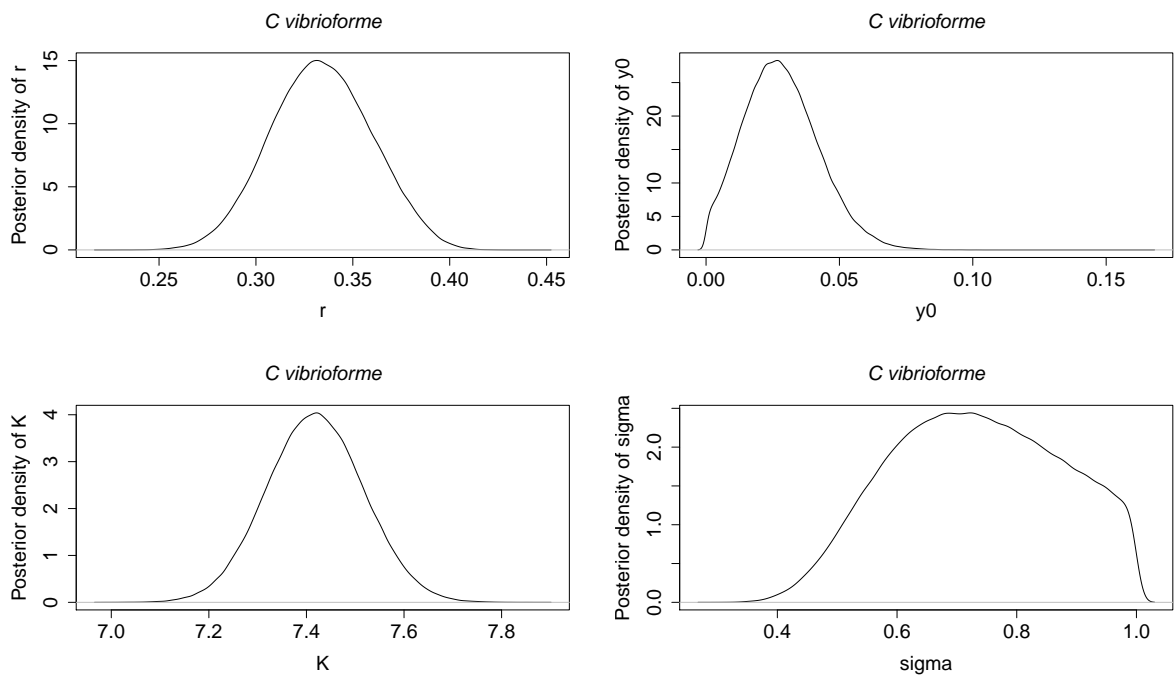

Figure 8.7: Posterior distributions for the model parameters of $C$. vibrioforme under the random logistic model 8.1 with gPC expansions. 

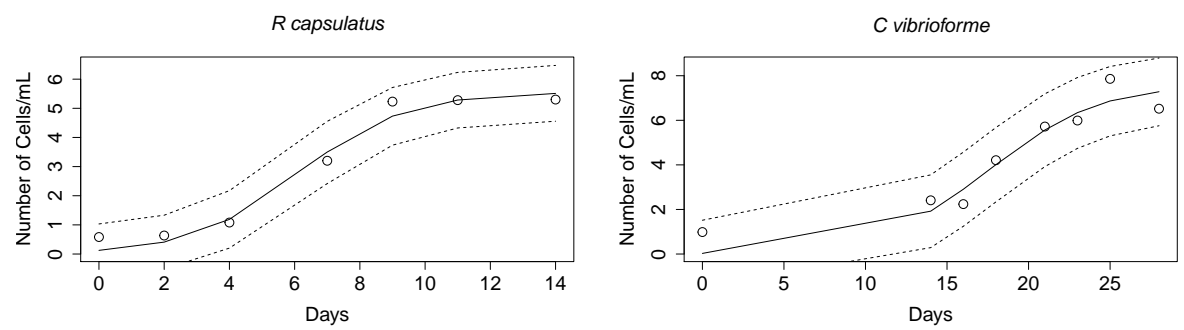

Figure 8.8: Model fitting for R. capsulatus (first plot) and C. vibrioforme (second plot) under the random logistic model 8.1 with gPC expansions. The real data is denoted by $\circ$, the fitting is given by the black continuous line and the 0.95 credible interval is drawn with dashed lines.

\begin{tabular}{|c|c|c|c|}
\hline \multicolumn{4}{|c|}{ Parameters for $R$. capsulatus } \\
\hline$r$ & $y_{0}$ & $K$ & residual \\
\hline 0.327079 & 0.479572 & 5.3322 & 0.0238877 \\
\hline \multicolumn{4}{|c|}{ Parameters for $C$. vibrioforme } \\
\hline$r$ & $y_{0}$ & $K$ & residual \\
\hline $0.0847 !$ & .6905 & 7.0967 & 1.85554 \\
\hline
\end{tabular}

Table 8.5: Parameters for $R$. capsulatus and C. vibrioforme under the new model 8.11.
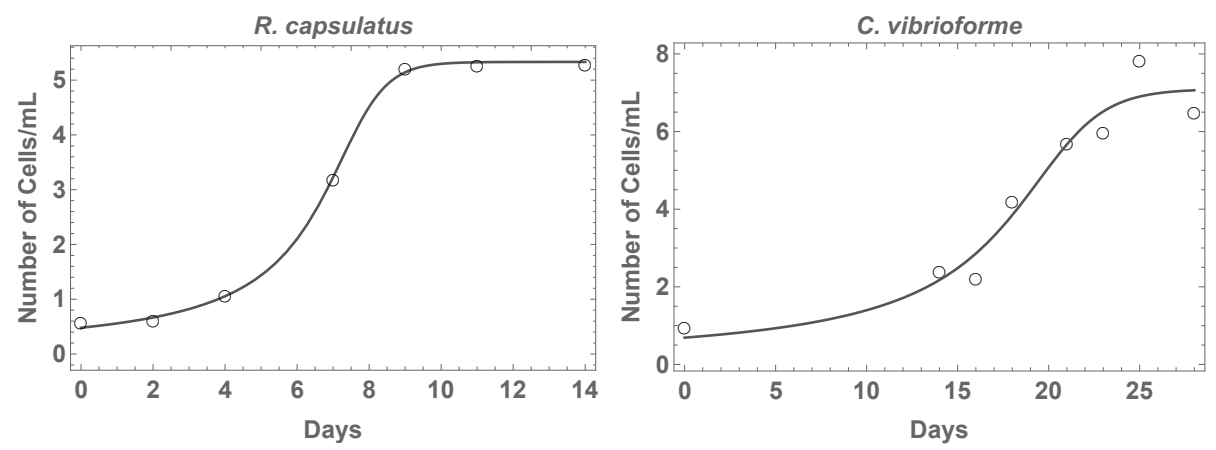

Figure 8.9: Least squares fitting of $R$. capsulatus (first plot) and C. vibrioforme (second plot) under the new model 8.11. The real data is denoted by $\circ$ and the fitting is given by the black continuous line. 
Parameters for R. capsulatus

\begin{tabular}{ccccc}
$r$ & $y_{0}$ & $K$ & $\delta$ & residual \\
\hline 0.43931 & 0.557972 & 5.60822 & 0.126042 & 0.0099536 \\
& Parameters for $C$. vibrioforme & \\
$r$ & $y_{0}$ & $K$ & $\delta$ & residual \\
\hline 0.116177 & 0.961639 & 7.6808 & 0.0694181 & 1.81952
\end{tabular}

Table 8.6: Parameters for R. capsulatus and C. vibrioforme under the new model 8.12).
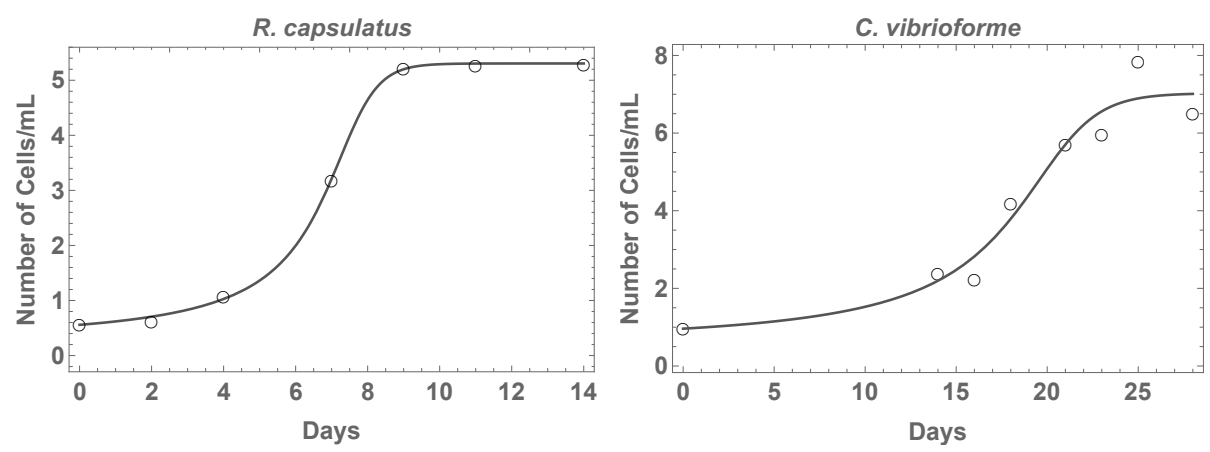

Figure 8.10: Least squares fitting of $R$. capsulatus (first plot) and C. vibrioforme (second plot) under the new model 8.12 . The real data is denoted by $\circ$ and the fitting is given by the black continuous line. 


\subsubsection{Random new model and gPC expansions}

Consider the new model 8.11) with random input coefficients. Using the Galerkin projection technique, we use Bayesian model $(8.9)-(8.10)$ to quantify the uncertainty of the solution process. We have set the prior distributions (8.13) for the parameters, with the values $\alpha_{r}=42.7927, \beta_{r}=130.8316$, $\alpha_{y_{0}}=91.99572, \beta_{y_{0}}=191.8288, \alpha_{K}=710.8089$ and $\beta_{K}=133.305$ for the $R$. capsulatus population; $\alpha_{r}=2.873249, \beta_{r}=33.90132, \alpha_{y_{0}}=190.7708$, $\beta_{y_{0}}=276.2396, \alpha_{K}=1259.079$ and $\beta_{K}=177.4175$ for the C. vibrioforme population; and the error $\sigma \sim \operatorname{Unif}(0,1)$. These values may be calculated as in the logistic model, by imposing the mean of the gamma distribution, $\alpha / \beta$, to be the least squares fitting estimate from Table 8.5. and the variance of the gamma distribution, $\alpha / \beta^{2}$, to be the desired dispersion for the parameter (this is more subjective). With a burnin period of 75,000 iterations, plus 150,000 samples for the model coefficients, and with two chains to assess convergence, the computational time was 47 seconds for the burnin period, plus 94 seconds for the latter 150,000 samples. In Table 8.7, Figure 8.11, Figure 8.12 and Figure 8.13, we show the results from the Bayesian inference with $p=2$. Observe that the solid lines from the figures behave similarly to the deterministic fittings from Figure 8.9. Moreover, the credible regions contain all data points, therefore the Bayesian model is appropriate for our data set.

Finally, consider the new model 8.12 with uncertainty. We combine the stochastic Galerkin projection technique and the Bayesian model (8.9)-(8.10) to quantify the uncertainty for the solution stochastic process. As prior distributions for $r, y_{0}, K, \delta$ and $\sigma$, we have set (8.13) for $r, y_{0}, K$ and $\sigma$, and $\delta \sim \operatorname{Gamma}\left(\alpha_{\delta}, \beta_{\delta}\right)$. For the numerical experiments, we have set $\alpha_{r}=77.19731, \beta_{r}=175.724, \alpha_{y_{0}}=124.5331, \beta_{y_{0}}=223.1888, \alpha_{K}=786.3033$, $\beta_{K}=140.2055, \alpha_{\delta}=6.354634$ and $\beta_{\delta}=50.4168$ for the $R$. capsulatus population; $\alpha_{r}=5.398838, \beta_{r}=46.4708, \alpha_{y_{0}}=369.8998, \beta_{y_{0}}=384.6556$, $\alpha_{K}=1474.867, \beta_{K}=192.02, \alpha_{\delta}=48.18873$ and $\beta_{\delta}=694.181$ for the $C$. vibrioforme population; and the error $\sigma \sim \operatorname{Unif}(0,1)$. As usual, we set a burnin period of 75,000 iterations, plus 150,000 samples for the model parameters, and with two chains to check convergence. The computational time was 47 seconds for the burnin period, plus 94 seconds for the latter 150,000 samples (the same time as the previous models, despite having one more parameter). In Table 8.8, Figure 8.14, Figure 8.15 and Figure 8.16, we present the results from the Bayesian inference with order of truncation $p=2$. Observe that the credible intervals contain all data measurements. 
Posterior distributions for $R$. capsulatus

\begin{tabular}{cccc} 
Parameter & Mean & sd & 0.95 interval \\
\hline$r$ & 0.3468 & 0.03955 & $(0.2661,0.3993)$ \\
$y_{0}$ & 0.4404 & 0.03182 & $(0.4037,0.4867)$ \\
$K$ & 5.352 & 0.162 & $(5.253,5.733)$
\end{tabular}

Posterior distributions for C. vibrioforme

\begin{tabular}{cccc} 
Parameter & Mean & sd & 0.95 interval \\
\hline$r$ & 0.05636 & 0.01112 & $(0.03632,0.07967)$ \\
$y_{0}$ & 0.7038 & 0.05447 & $(0.602,0.815)$ \\
$K$ & 7.369 & 0.1535 & $(7.008,7.608)$
\end{tabular}

Table 8.7: Descriptive table for the posterior distributions of the parameters for $R$. capsulatus and $C$. vibrioforme under the new model (8.11) with gPC expansions.
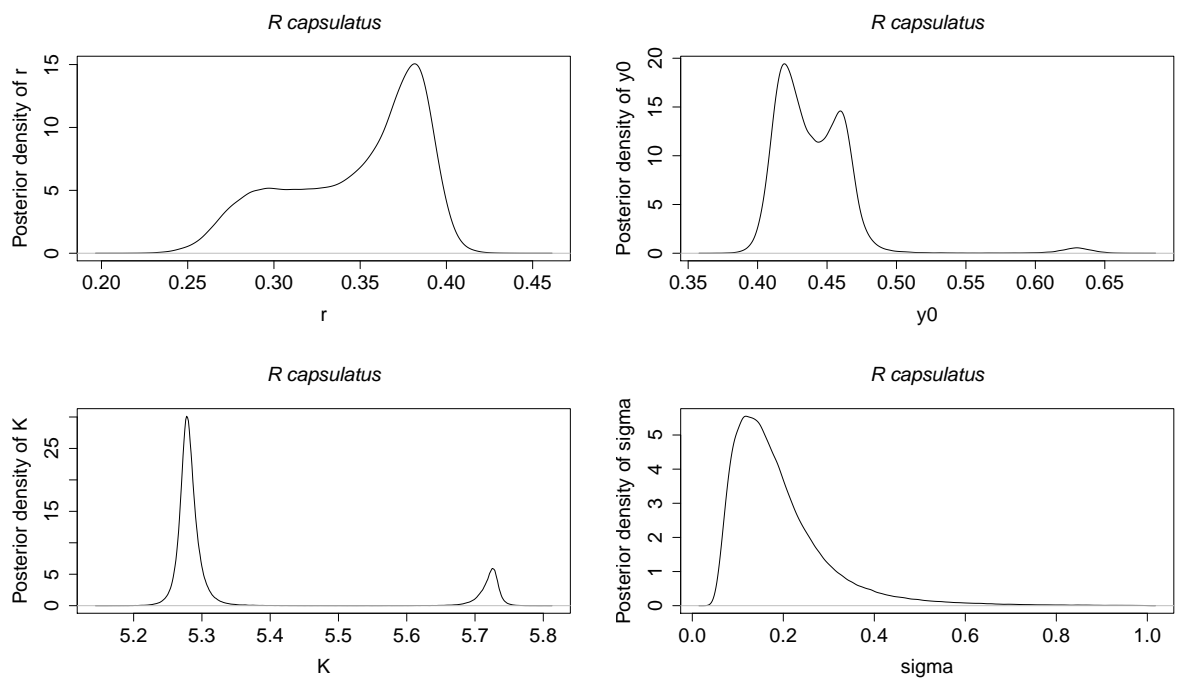

Figure 8.11: Posterior distributions for the model parameters of $R$. capsulatus under the new model 8.11 with gPC expansions. 

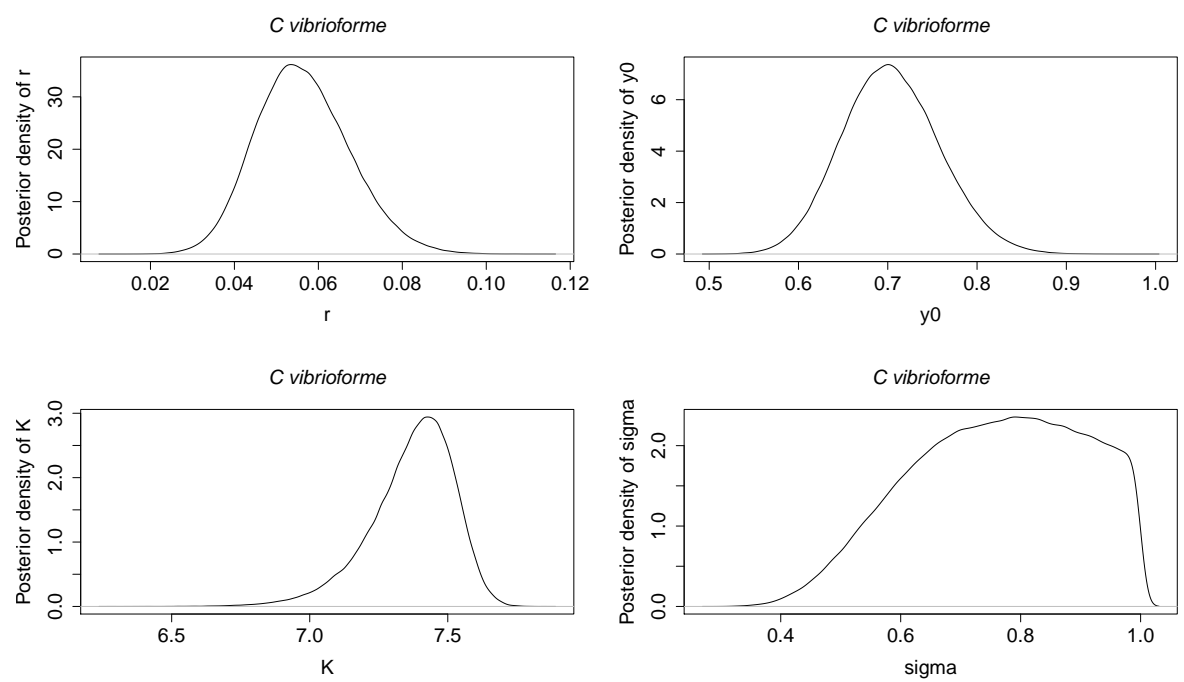

Figure 8.12: Posterior distributions for the model parameters of $C$. vibrioforme under the new model 8.11 with gPC expansions.
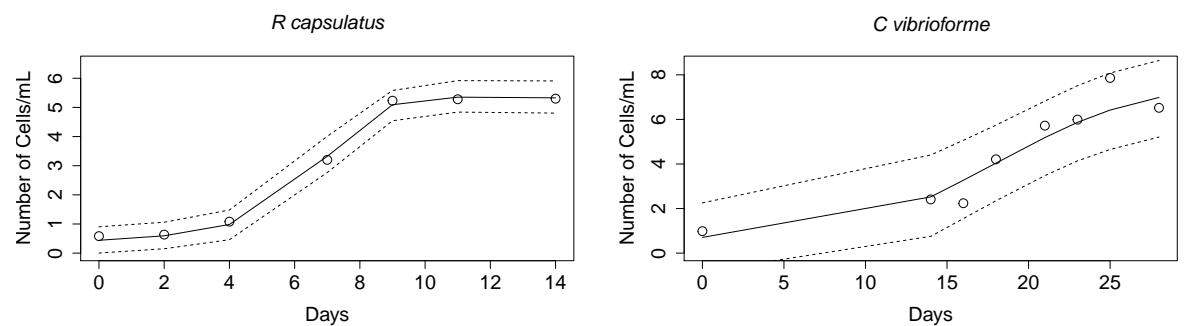

Figure 8.13: Model fitting for $R$. capsulatus (first plot) and C. vibrioforme (second plot) under the new model 8.11) with gPC expansions. The real data is denoted by $\circ$, the fitting is given by the black continuous line and the 0.95 credible interval is drawn with dashed lines. 

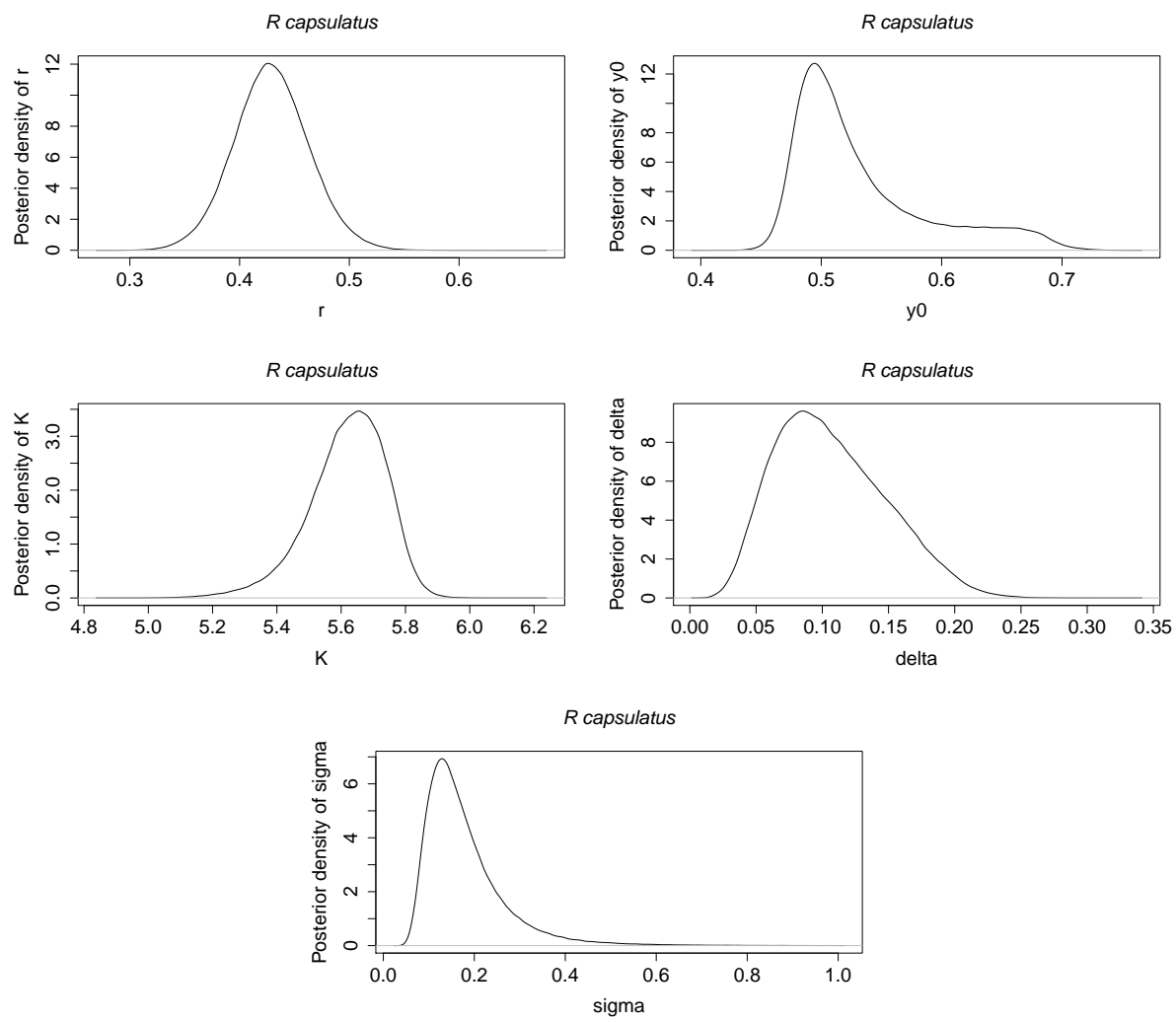

Figure 8.14: Posterior distributions for the model parameters of $R$. capsulatus under the new model 8.12 with gPC expansions. 

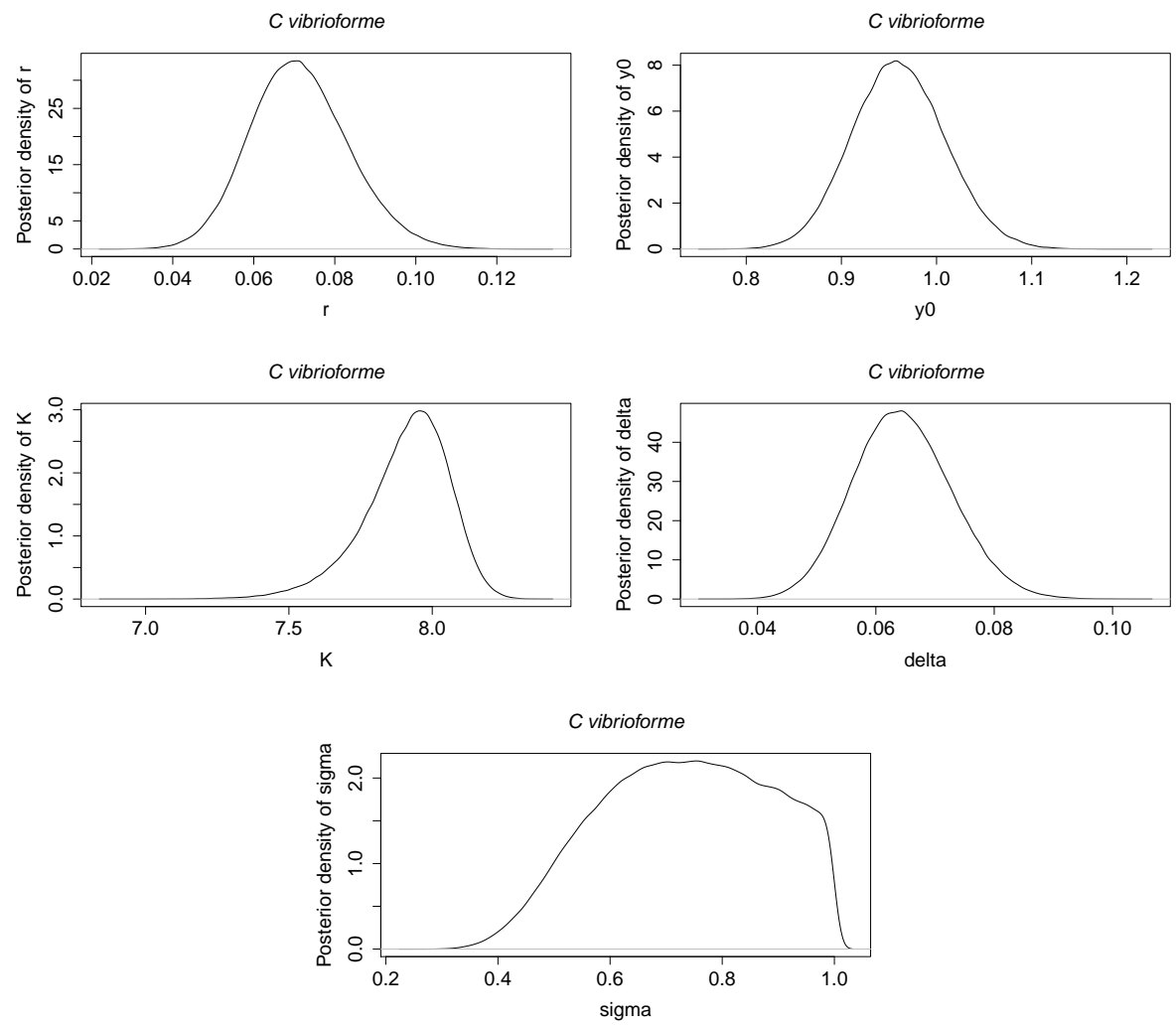

Figure 8.15: Posterior distributions for the model parameters of $C$. vibrioforme under the new model 8.12 with gPC expansions. 
Posterior distributions for R. capsulatus

\begin{tabular}{|c|c|c|c|}
\hline Parameter & Mean & sd & 0.95 interval \\
\hline$r$ & 0.4291 & 0.03444 & $(0.3621,0.4986)$ \\
\hline$y_{0}$ & 0.5322 & 0.05639 & $(0.467,0.6763)$ \\
\hline$K$ & 5.618 & 0.121 & $(5.339,5.815)$ \\
\hline$\delta$ & 0.1068 & 0.04146 & $(0.04051,0.1954)$ \\
\hline \multicolumn{4}{|c|}{ Posterior distributions for $C$. vibrioforme } \\
\hline Parameter & Mean & sd & 0.95 interval \\
\hline$r$ & 0.07133 & 0.01208 & $(0.04899,0.09645)$ \\
\hline$y_{0}$ & 0.9604 & 0.04884 & $(0.8672,1.058)$ \\
\hline$K$ & 7.908 & 0.1513 & $(7.554,8.152)$ \\
\hline$\delta$ & 0.06487 & 0.008284 & $(0.04913,0.08149)$ \\
\hline
\end{tabular}

Table 8.8: Descriptive table for the posterior distributions of the parameters for $R$. capsulatus and $C$. vibrioforme under the new model 8.12 with gPC expansions.
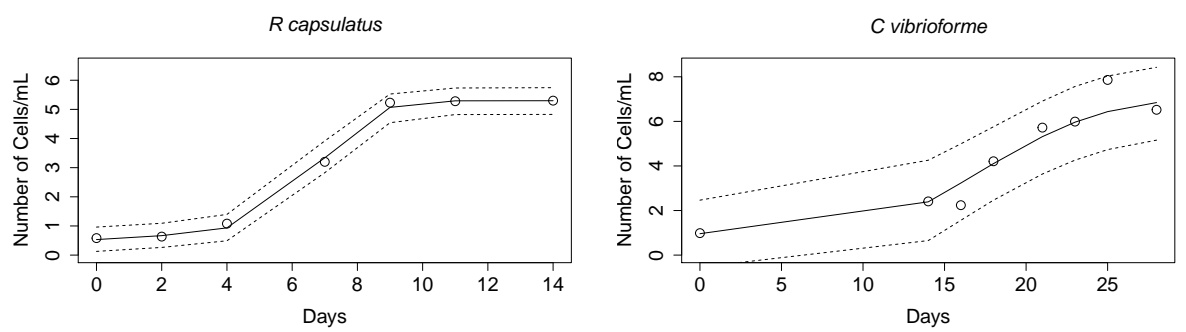

Figure 8.16: Model fitting for $R$. capsulatus (first plot) and C. vibrioforme (second plot) under the new model 8.12 with gPC expansions. The real data is denoted by $\circ$, the fitting is given by the black continuous line and the 0.95 credible interval is drawn with dashed lines. 


\subsection{Conclusions}

Mathematical models for biological population growth are important to understand and generalize the results to other situations and to make predictions. Due to the inherent uncertainty associated to biological phenomena (errors in the laboratory experiments, lack of information, missed data, etc.), randomness must be introduced in the model. In this chapter, we have studied a random differential model of growth of anaerobic photosynthetic bacteria. In the laboratory experiment, actual measurements for two species of bacteria were collected: $R$. capsulatus and $C$. vibrioforme. A previous article by D. Stanescu et al. [Electronic Transactions on Numerical Analysis, 34, 44-58 (2009)] considered a logistic model to explain bacterial growth in both populations, based on Malthusian exponential growth model and competitiveness when there is scarcity of nutrients (mainly light and $\mathrm{CO}_{2}$ ). In this chapter, we have improved the fit of the deterministic logistic model by assuming that the growth rate is proportional to the squared abundance of microorganisms, and by taking into account the start of the death phase in the kinetic curve. Instead of introducing uncertainty into the model by using arbitrary distributions for the coefficients, we have utilized a Bayesian inverse approach for parameter estimation. Since our model does not have an explicit solution, one would need to solve it for each sampling point of the Markov Chain Monte Carlo algorithm. However, gPC expansions together with the stochastic Galerkin projection technique have allowed accelerating the Bayesian inference. Spectral convergence of the Galerkin projection implies exponential convergence rate for the corresponding prior distributions in the sense of the KullbackLeibler divergence, even when the variance of the error is supposed random with a prior distribution. This fact has permitted obtaining reliable results for the posterior distributions of the coefficients and the posterior predictive distribution, so that it is possible to computationally quantify the uncertainty for the bacteria population growth.

\section{Acknowledgements}

This work has been supported by the Spanish Ministerio de Economía y Competitividad grant MTM2017-89664-P.

The main results of this chapter have been published in [22]. 



\section{Chapter 9}

\section{Conclusions}

In this thesis, we have studied mathematical models with randomness, namely random ordinary and partial differential equations. The models have random input parameters and the solution is a stochastic process. In general, randomness is motivated by incomplete knowledge of the underlying true physics and error measurements. In this context, uncertainty quantification consists in analyzing the propagation of uncertainty from the inputs to the system output. One aims at calculating the main statistics of the solution, say the expectation and the variance, and even the probability density function.

The statistics of the solution can be estimated by employing Monte Carlo simulation. Another method, relying on spectral expansions, is the gPCbased Galerkin projection method. For the computation of the probability density function, the RVT method gives the exact representation of the density function when a simple closed-form solution exists. Otherwise, stochastic approximations of the solution are constructed to derive a sequence of density functions that tends to the target density. In this regard, Karhunen-Loève and gPC expansions and finite difference discretizations give approximations of the solution and allow for approximating its density function. The convergence analysis is not straightforward, in general, and different mathematical tools must be employed to prove rigorously the convergence of the sequence of density functions. 
For different mathematical models that arise in Physics, Biology, Epidemiology, etc. we have studied their random counterparts and we have computed faithful approximations to the probability density function of the solution. In Chapter 2, we dealt with the damped pendulum random differential equations. In Chapter 3, we addressed the logistic growth random differential equation. Chapter 4 was devoted to a comprehensive analysis of the random Bertalanffy model. These three chapters used Karhunen-Loève expansions as the approximations tool. In Chapter 5, we employed gPC expansions for the approximation of the density function, in the context of epidemic models with one input random parameter. In Chapters 6 and 7, we considered the heat partial differential equation with random Dirichlet boundary conditions; density approximations were constructed from Karhunen-Loève expansions and a finite difference numerical method, respectively.

In all of the previous cases, it was assumed that the input random parameters had a probability distribution already set. But in modeling, an important problem is to determine the probability distribution of the parameters from the collected data. This is an inverse problem. In Bayesian inference, from a prior distribution of the parameters (noninformative, or based on experience or other studies) and from the data (its likelihood), simulations from the posterior distribution of the parameters are obtained. When a simple closedform expression of the model solution is not available, a hybrid method that combines Bayesian inference and gPC expansions accelerates the simulation from the posterior distribution. Chapter 8 used these ideas to model bacteria growth.

Uncertainty quantification for stochastic systems is an active field under study, as they allow for more reliable models of complex phenomena. In the future, we will devote effort on the study of new randomized mathematical models. 


\section{Bibliography}

[1] A. Ambrosetti and G. Prodi. A Primer of Nonlinear Analysis. Cambridge Studies in Advanced Mathematics. New York: Cambridge University Press, 1993. ISBN: 9780521485739. (Cited on page 17.)

[2] T.M. Apostol. Mathematical Analysis. Ed. 2. New York: Pearson, 1976. (Cited on page 111.)

[3] D. Applebaum, B.V.R. Bhat, J. Kustermans, and J.M. Lindsay. Quantum Independent Increment Processes I, From Classical Probability to Quantum Stochastic Calculus. Lecture Notes in Mathematics. Berlin Heidelberg: Springer, 2005. (Cited on page 113.)

[4] J.R. Banavar, J. Damuth, A. Maritan, and A. Rinaldo. "Ontogenetic growth. Modelling universality and scaling". In: Nature 420 (2002), p. 626. DOI: 10.1038/420626a. (Cited on page 56.)

[5] A. Berman and R.J. Plemmons. Nonnegative Matrices in the Mathematical Sciences. New York: SIAM, 1994. (Cited on page 140.)

[6] A.T. Bharucha-Reid. On the theory of random equations. In: Proc. Sympos. Appl. Math. 16 (1964), pp. 40-69. (Cited on pages 6 and 40.)

[7] L.v. Bertalanffy. "Quantitative laws in metabolism and growth". In: $Q$. Rev. Biol. 32.3 (1957), pp. 217-231. (Cited on page 56.)

[8] P. Billingsley. Probability and Measure. Ed. 3. Wiley Series in Probability and Mathematical Statistics. India: Wiley, 1995. (Cited on pages 9, 65. $68,74,106,112$, and 141.) 
[9] J.L. Bogdanoff, J.E. Goldberg, and M.C. Bernard. "Response of a simple structure to a random earthquake-type disturbance". In: B. Seismol. Soc. Am. 51.2 (1961), pp. 293-310. (Cited on page 27.)

[10] F. Brauer and C. Castillo-Chávez. Mathematical Models in Population Biology and Epidemiology. Springer Science + Business Media, 2012. (Cited on page 87.)

[11] H. Brezis. Functional Analysis, Sobolev Spaces and Partial Differential Equations. Universitext. New York: Springer, 2010. (Cited on pages 17. 45 , and 46 ,

[12] C. Burgos, J. Calatayud, J.-C. Cortés, and A. Navarro-Quiles. "A full probabilistic solution of the random linear fractional differential equation via the random variable transformation technique". In: Math. Meth. Appl. Sci. 41.18 (2018), pp. 9037-9047. DOI: 10.1002/mma.4881. (Cited on page 41.)

[13] C. Burgos, J. Calatayud, J.-C. Cortés, and L. Villafuerte. "Solving a class of random non-autonomous linear fractional differential equations by means of a generalized mean square convergent power series". In: Appl. Math. Lett. 78 (2018), pp. 95-104. DOI: 10.1016/j.aml.2017.11.009. (Cited on page 86.)

[14] J. Calatayud, B.M. Chen-Charpentier, J.-C. Cortés, and M. Jornet. "Combining polynomial chaos expansions and the random variable transformation technique to approximate the density function of stochastic problems, including some epidemiological models". In: Symmetry 11.1 (2019), p. 43. DOI: 10.3390/sym11010043. (Cited on pages 2 and 100.)

[15] J. Calatayud, J.-C. Cortés, J.A. Díaz, and M. Jornet. "Constructing reliable approximations of the probability density function to the random heat PDE via a finite difference scheme". In: Appl. Numer. Math. 151 (2020), pp. 413-424. DOI: 10.1016/j.apnum.2020.01.012. (Cited on page 148,)

[16] J. Calatayud, J.-C. Cortés, and M. Jornet. "On the approximation of the probability density function of the randomized heat equation". (2018) https://arxiv.org/pdf/1802.04190.pdf. (Cited on pages 12, 20, 103 $104,105,106,107,109,118,122,135,136$, and 142.)

[17] J. Calatayud, J.-C. Cortés, and M. Jornet. "The damped pendulum random differential equation: A comprehensive stochastic analysis via the computation of the probability density function". In: Physica A 512 (2018), pp. 261-279. DOI: 10.1016/j.physa.2018.08.024. (Cited on pages 2, 37, 41 . 45, 46, 53, and 87.)

[18] J. Calatayud, J.-C. Cortés, and M. Jornet. "Improving the approximation of the probability density function of random non-autonomous logistic- 
type differential equations. In: Math. Method. Appl. Sci. (2019). DOI: 10.1002/mma.5834. (Cited on page 54.)

[19] J. Calatayud, J.-C. Cortés, and M. Jornet. "Uncertainty quantification for random parabolic equations with nonhomogeneous boundary conditions on a bounded domain via the approximation of the probability density function". In: Math. Method. Appl. Sci. 42.17 (2019), pp. 5649-5667. DOI: 10.1002/mma.5333. (Cited on pages 2, 41, 87, and 131,)

[20] J. Calatayud, J.-C. Cortés, and M. Jornet. "Approximate solutions of randomized non-autonomous complete linear differential equations via probability density functions". In: Electron. J. Differ. Eq. 2019.85 (2019), pp. 140. (Cited on pages 41, 46, 56, 57, 58, 60, 62, 63, 64, 65, 66, 67, 68, and 69, )

[21] J. Calatayud, J.-C. Cortés, and M. Jornet. "On the convergence of adaptive gPC for non-linear random difference equations: Theoretical analysis and some practical recommendations". In: J. Nonlinear Sci. App., 11.9 (2018), pp. 1077-1084. DOI: 10.22436/jnsa.011.09.06. (Cited on pages 1. 83, 85, 92 , and 94 .)

[22] J. Calatayud, J.-C. Cortés, and M. Jornet. "Improvement of random coefficient differential models of growth of anaerobic photosynthetic bacteria by combining Bayesian inference and gPC". In: Math. Method. Appl. Sci. (2019). DOI: 10.1002/mma.5546. (Cited on page 179.)

[23] J. Calatayud, J.-C. Cortés, M. Jornet, and A. Navarro-Quiles. "Solving Random Ordinary and Partial Differential Equations through the Probability Density Function. Theory and Computing with Applications". Understanding Complex Systems. New York: Springer International Publishing AG (Springer Nature), 2019. Chap 15 Modern Mathematics and Mechanics. Fundamentals, Problems and Challenges, pp. 261-282. ISBN: 978-3-319-96755-4. (Cited on pages 2, 80, and 83.)

[24] J. Calatayud, J.-C. Cortés, M. Jornet, and R.J. Villanueva. "Computational uncertainty quantification for random time-discrete epidemiological models using adaptive gPC". In: Math. Method. Appl. Sci. (2018), pp. 110. DOI: 10.1002/mma.5315. (Cited on pages 1, 83, 86, 94, and 95.)

[25] B.P. Carlin and T.A. Louis. Bayes and Empirical Bayes Methods for Data Analysis. Chapman and Hall/CRC, 2010. (Cited on page 157.)

[26] M.C. Casabán, R. Company, J.-C. Cortés, and L. Jódar. "Solving the random diffusion model in an infinite medium: A mean square approach". In: Appl. Math. Model. 38.24 (2014), pp. 5922-5933. DOI: 10.1016/j.apm.2014.04.063. (Cited on pages 102, 134, and 135.)

[27] M.C. Casabán, J.-C. Cortés, and L. Jódar. "Solving random mixed heat problems: A random integral transform approach". In: J. Comput. Appl. 
Math. 291 (2016), pp. 5-19. DOI: 10.1016/jcam.2014.09.021. (Cited on page 102.)

[28] M.C. Casabán, J.-C. Cortés, A. Navarro-Quiles, J.V. Romero, M.D. Roselló, and R.-J. Villanueva. "Computing probabilistic solutions of the Bernoulli random differential equation". In: J. Comput. Appl. Math. 309 (2017), pp. 396-407. DOI: 10.1016/j.cam.2016.02.034. (Cited on page 56.)

[29] M.C. Casabán, J.-C. Cortés, J.V. Romero, and M.D. Roselló. "Probabilistic solution of random SI-type epidemiological models using the Random Variable Transformation technique". In: Commun. Nonlinear Sci. Numer. Simulat. 24.1-3 (2015), pp. 86-97. DOI: 10.1016/j.cnsns.2014.12.016. (Cited on pages 2, 80, 83, and 160,)

[30] M.C. Casabán, J.-C. Cortés, J.V. Romero, and M.D. Roselló. "A comprehensive probabilistic solution of random SIS-type epidemiological models using the random variable transformation technique". In: Commun. Nonlinear Sci. Numer. Simulat. 32 (2016), pp. 199-210. DOI: 10.1016/j.cnsns.2015.08.009. (Cited on pages 80 and 95.)

[31] G. Casella. "An introduction to empirical Bayes data analysis". In: Am Stat 39.2 (1985), pp. 83-87. (Cited on page 157.)

[32] T.K. Caughey. "On the response of non-linear oscillators to stochastic excitation". In: Probabilist. Eng. Mech. 1.1 (1986), pp. 2-4. DOI: 10.1016/0266-8920(86)90003-2. (Cited on page 28.)

[33] B.-M. Chen-Charpentier, J.-C. Cortés, J.-A. Licea, J.-V. Romero, M.D. Roselló, F.-J. Santonja, and R.-J. Villanueva. "Constructing adaptive generalized polynomial chaos method to measure the uncertainty in continuous models: A computational approach". In: Math. Comput. Simulat. 109 (2015), pp. 113-129. DOI: 10.1016/j.matcom.2014.09.002. (Cited on pages 1, 82, 83, 86, 87, and 160.)

[34] B.-M. Chen-Charpentier, J.-C. Cortés, J.-V. Romero, and M.-D. Roselló. "Some recommendations for applying gPC (generalized polynomial chaos) to modeling: An analysis through the Airy random differential equation". In: Appl. Math. Comput. 219.9 (2013), pp. 4208-4218. DOI: 10.1016/j.amc.2012.11.007. (Cited on page 80.)

[35] B.M. Chen-Charpentier and D. Stanescu. "Epidemic models with random coefficients". In: Math. Comput. Model. 52.7-8 (2010), pp. 1004-1010. DOI: 10.1016/j.mcm.2010.01.014. (Cited on pages 1, 83, and 86.)

[36] R. Cherniha and V. Davydovych. Nonlinear Reaction-Diffusion Systems: Conditional Symmetry, Exact Solutions and their Applications in Biology. Vol. 2196. Lecture Notes in Mathematics. Springer, 2017. (Cited on page 91.) 
[37] R. Chiba. "Stochastic heat conduction analysis of a functionally graded annular disc with spatially random heat transfer coefficients". In: Appl. Math. Model. 33.1 (2009), pp. 507-523. DOI: 10.1016/j.apm.2007.11.014. (Cited on page 102.)

[38] A. Corberán-Vallet, F.J. Santonja, M. Jornet-Sanz, and R.J. Villanueva. "Modeling chickenpox dynamics with a discrete time bayesian stochastic compartmental model". In: Complexity 2018 (2018). DOI: 10.1155/2018/3060368. (Cited on page 151.)

[39] J.-C. Cortés, L. Jódar, M.D. Roselló, and L. Villafuerte. "Solving initial and two-point boundary value linear random differential equations: A mean square approach". In: Appl. Math. Comput. 219.4 (2012), pp. 22042211. DOI: 10.1016/j.amc.2012.08.066. (Cited on page 59.)

[40] J.-C. Cortés, L. Jódar, and L. Villafuerte. Random linear-quadratic mathematical models: computing explicit solutions and applications. In: Comput. Math. Appl. 79.7 (2009), pp. 2016-90. DOI: 10.1016/j.matcom.2008.11.008. (Cited on page 40.)

[41] J.-C. Cortés, L. Jódar, L. Villafuerte, and R.J. Villanueva. "Computing mean square approximations of random diffusion models with source term". In: Math. Comput. Simulat. 76.1-3 (2006), pp. 44-48. DOI: j.matcom.2007.01.020. (Cited on page 135.)

[42] J.-C. Cortés, A. Navarro-Quiles, J.V. Romero, and M.D. Roselló. "Analysis of random non-autonomous logistic-type differential equations via the Karhunen-Loève expansion and the Random Variable Transformation technique". In: Commun. Nonlinear Sci. Numer. Simulat. 72 (2019), pp. 121138. DOI: 10.1016/j.cnsns.2018.12.013. (Cited on pages 40, 41, 44, 45, 46. 47, 48, 49, 52, and 53.)

[43] J.-C. Cortés, A. Navarro-Quiles, J.V. Romero, and M.D. Roselló. "Computing the probability density function of non-autonomous first-order linear homogeneous differential equations with uncertainty". In: J. Comput. Appl. Math. 337 (2018), pp. 190-208. DOI: 10.1016/j.cam.2018.01.015. (Cited on page 41.)

[44] J.-C. Cortés, A. Navarro-Quiles, J.V. Romero, and M.D. Roselló. "Full solution of random autonomous first-order linear systems of difference equations. Application to construct random phase portrait for planar systems". In: Appl. Math. Lett. 68 (2017), pp. 150-156. DOI: 10.1016/j.aml.2016.12.015. (Cited on pages 2 and 83.)

[45] J.-C. Cortés, A. Navarro-Quiles, J.V. Romero, and M.D. Roselló. "Solving second-order linear differential equations with random analytic coefficients about ordinary points: A full probabilistic solution by the first probability 
density function". In: Appl. Math. Comput. 331 (2018), pp. 33-45. DOI: 10.1016/j.amc.2018.02.051. (Cited on pages 2 and 87.)

[46] J.-C. Cortés, J.-V. Romero, M.-D. Roselló, and R.-J. Villanueva. "Improving adaptive generalized polynomial chaos method to solve nonlinear random differential equations by the random variable transformation technique". In: Commun. Nonlinear Sci. Numer. Simul. 50 (2017), pp. 115. DOI: 10.1016/j.cnsns.2017.02.011. (Cited on pages 1, 80, 82, 83, 139 and 160 )

[47] J.-C. Cortés, P. Sevilla, and L. Jódar. "Analytic-numerical approximating processes of diffusion equation with data uncertainty". In: Comput. Math. Appl. 49.7-8 (2005), pp. 1255-1266. DOI: j.camwa.2004.05.015. (Cited on pages xxii, xxiii, 134, 135, 144, 145, 146, 147, and 148,)

[48] J.-C. Cortés, P. Sevilla, and L. Jódar. "Constructing approximate diffusion processes with uncertain data". In: Math. Comput. Simulat. 73.1-4 (2006), pp. 125-132. DOI: j.matcom.2006.06.009. (Cited on page 135.)

[49] T. Crestaux, O. Le Maître, and J.M. Martínez. "Polynomial chaos expansion for sensitivity analysis". In: Reliab. Eng. Syst. Safe. 94.7 (2009), pp. 1161-1172. DOI: 10.1016/j.ress.2008.10.008. (Cited on page 80.)

[50] S. Depaoli, J.P. Clifton, and P.R. Cobb. "Just Another Gibbs Sampler (JAGS): Flexible Software for MCMC Implementation". In: J. Educ. Behav. Stat. 41.6 (2016), pp. 628-649. DOI: 10.3102/1076998616664876. (Cited on page 158.)

[51] F.A. Dorini, N. Bobko, and L.B. Dorini. "A note on the logistic equation subject to uncertainty in parameters". In: Comput. Appl. Math. 37.2 (2018), pp. 1496-1506. DOI: 10.1007/s40314-016-0409-6. (Cited on page 41.)

[52] F.A. Dorini, M.S. Cecconello, and M.B. Dorini. "On the logistic equation subject to uncertainties in the environmental carrying capacity and initial population density". In: Commun. Nonlinear Sci. Numer. Simul. 33 (2016), pp. 160-173. DOI: 10.1016/j.cnsns.2015.09.009. (Cited on pages 2 , 41, 80, 83, and 139 ,

[53] F.A. Dorini and M.C.C. Cunha. "Statistical moments of the random linear transport equation". In: J. Comput. Phys. 227.19 (2008), pp. 8541-8550. DOI: $10.1016 /$ j.jcp.2008.06.002. (Cited on pages 2, 6, 80, 83, and 103.)

[54] F.A. Dorini and M.C.C. Cunha. "On the linear advection equation subject to random velocity fields". In: Math. Comput. Simulat. 82.4 (2011), pp. 679-690. DOI: 10.1016/j.matcom.2011.10.008. (Cited on page6.) 
[55] F.A. Dorini, F. Furtado, and M.C.C. Cunha. "On the evaluation of moments for solute transport by random velocity fields". In: Appl. Numer. Math. 59.12 (2009), pp. 2994-2998. DOI: 10.1016/j.apnum.2009.07.006. (Cited on page 6.)

[56] R. Dutta. Fundamentals of Biochemical Engineering. India: Springer, 2008. (Cited on page 150.)

[57] M.A. El-Tawil. "The approximate solutions of some stochastic differential equations using transformations". In: Appl. Math. Comput. 164 (2005), pp. 167-178. DOI: 10.1016/j.amc.2004.04.062. (Cited on pages 2, 87. and 135 .)

[58] O.G. Ernst, A. Mugler, H.J. Starkloff, and E. Ullmann. "On the convergence of generalized polynomial chaos expansions". In: ESAIM-Math. Model. Num. 46.2 (2012), pp. 317-339. DOI: 10.1051/m2an/2011045. (Cited on pages 82 and 160 .)

[59] G. Falsone and D. Settineri. "On the application of the probability transformation method for the analysis of discretized structures with uncertain proprieties". In: Probabilist. Eng. Mech. 35 (2014), pp. 44-51. DOI: j.probengmech.2013.10.001. (Cited on page6.)

[60] G. Fishman. Monte Carlo: Concepts, Algorithms, and Applications. Springer Series in Operations Research and Financial Engineering. New York: Springer Science \& Business Media, 2013. (Cited on pages 1 and 141.)

[61] H. Fujikawa, A. Kai, and S. Morozumi. "A new logistic model for bacterial growth". In: J. Food. Hyg. Soc. Jpn. 44.3 (2003), pp. 155-160. (Cited on page 150.)

[62] L. Giraud, J. Langou, and M. Rozloznik. "The loss of orthogonality in the Gram-Schmidt orthogonalization process". In: Comput. Math. Appl. 50.7 (2005), pp. 1069-1075. DOI: 10.1016/j.camwa.2005.08.009. (Cited on pages 85 and 160 .)

[63] L.R. Glicksman and J.H. Lienhard. Modeling and Approximation in Heat Transfer. Cambridge University Press, 2016. (Cited on page 134.)

[64] J.E. Goldberg, J.L. Bogdanoff, and D.R. Sharpe. "The response of simple nonlinear systems to a random disturbance of the earthquake type". In: B. Seismol. Soc. Am. 54.1 (1964), pp. 263-276. (Cited on page 27.)

[65] G. Grimmett and D. Stirzaker. Probability and Random Processes. Clarendon Press, Oxford, 2000. (Cited on page 140.)

[66] A. Gut. Probability: A Graduate Course. Springer Texts in Statistics. New York: Springer, 2005. ISBN: 9780387228334. (Cited on page 30.) 
[67] K. Hadeler. Topics in Mathematical Biology. Springer, 2017. (Cited on page 95.)

[68] J.K. Hale. Ordinary Differential Equations. Ed. 2. Malabar: Robert E. Krieger Publishing Company, 1980. (Cited on pages 8 and 58.)

[69] H.W. Hethcote. "The mathematics of infectious diseases". In: SIAM Rev. 42.4 (2000), pp. 599-653. DOI: 10.1137/S0036144500371907. (Cited on page 150 .)

[70] H. Holden, B. Øksendal, J. Uboe, and T. Zhang. Stochastic Partial Differential Equations: A Modeling, White Noise Functional Approach. Ed. 2. Probability and Its Applications. New York: Springer Science+Business Media LLC, 2010. (Cited on page 102.)

[71] M.H. Heydari, M.R. Hooshmandasl, C. Cattani, and F.M. Maalek Ghainiab. "An efficient computational method for solving nonlinear stochastic Itô integral equations: Application for stochastic problems in physics". In: J. Comput. Phys. 283 (2015), pp. 148-168. DOI: 10.1016/j.jcp.2014.11.042. (Cited on page 6.)

[72] A. Hussein and M.M. Selim. "Solution of the stochastic radiative transfer equation with Rayleigh scattering using RVT technique". In: Appl. Math. Comput. 218 (2012), pp. 7193-7203. DOI: 10.1016/j.amc.2011.12.088. (Cited on pages 2, 6, 80, 83, 103, and 139, )

[73] A. Hussein and M.M. Selim. "Solution of the stochastic generalized shallow-water wave equation using RVT technique". In: Eur. Phys. J. Plus 130.249 (2015). DOI: 10.1140/epjp/i2015-15249-3. (Cited on pages 2 . 6. 80, 83, and 103.)

[74] A. Hussein and M.M. Selim. "A general analytical solution for the stochastic Milne problem using Karhunen-Loève (K-L) expansion". In: J. Quant. Spectrosc. Ra. 125 (2013), pp. 84-92. DOI: 10.1016/j.jqsrt.2013.03.018. (Cited on pages 2, 6, 80, and 83,

[75] S. Jin and H. Lu. "An asymptotic-preserving stochastic Galerkin method for the radiative heat transfer equations with random inputs and diffusive scalings". In: J. Comput. Phys. 334 (2017), pp. 182-206. DOI: 10.1016/j.jcp.2016.12.033. (Cited on page 102.)

[76] S. Jin, D. Xiu, and X. Zhu. "Asymptotic-preserving methods for hyperbolic and transport equations with random inputs and diffusive scalings". In: J. Comput. Phys. 289 (2015), pp. 35-52. DOI: 10.1016/j.jcp.2015.02.023. (Cited on page 102.)

[77] N.L. Johnson, S. Kotz, and N. Balakrishnan. Continuous Univariate Distributions. Vol. 1. Wiley, 1994. (Cited on page 27.) 
[78] T.R. Johnson and K.M. Kuhn. "Bayesian Thurstonian models for ranking data using JAGS". In: Behav. Res. Methods 45.3 (2013), pp. 857-872. DOI: 10.3758/s13428-012-0300-3. (Cited on page 158.)

[79] A. Juška, G. Gedminienè, and R. Ivanec. "Growth of microbial populations. Mathematical modeling, laboratory exercises, and model-based data analysis". In: Biochem. Mol. Biol. Edu. 34.6 (2006), pp. 417-422. DOI: 10.1002/bmb.2006.494034062669. (Cited on page 150.)

[80] B. Kegan and R.W. West. "Modeling the simple epidemic with deterministic differential equations and random initial conditions". In: Math. Biosci. 194.2 (2005), pp. 217-231. DOI: 10.1016/j.mbs.2005.02.002. (Cited on page 80.)

[81] W. Kliemann. Nonlinear Dynamics and Stochastic Mechanics. CRC Press, 2018. (Cited on page 28,)

[82] P. Kloeden and E. Platen. Numerical Solution of Stochastic Differential Equations. Berlin and Heidelberg: Springer-Verlag, 2011. (Cited on page 102, )

[83] H. Kobayashi, B.L. Mark, and W. Turin. Probability, Random Processes, and Statistical Analysis. Cambridge University Press, 2011. ISBN: 9781139183383. (Cited on pages 60 and 83.)

[84] J.F. Lawless. Truncated Distributions. Wiley StatsRef: Statistics Reference Online, 2014. (Cited on page 119.)

[85] E.L. Lehmann and G. Casella. Theory of Point Estimation. Springer Science \& Business Media, 2006. (Cited on page 157.)

[86] E. Lesaffre and A.B. Lawson. Bayesian Biostatistics. Statistics in Practice. New York: Wiley, 2012. (Cited on pages 151, 157, 158, 159, and 166.)

[87] S.A: Levin, T.G. Hallam, and L.J. Gross. Applied Mathematical Ecology. Vol. 18. Springer Science \& Business Media, 2012. (Cited on page 150.)

[88] J.A. Licea, L. Villafuerte, and B.M. Chen-Charpentier. "Analytic and numerical solutions of a Riccati differential equation with random coefficients". In: J. Comput. Appl. Math. 239 (2013), pp. 208-219. DOI: 10.1016/j.cam.2012.09.040. (Cited on page 40.)

[89] G.J. Lord, C.E. Powell, and T. Shardlow. An Introduction to Computational Stochastic PDEs. New York: Cambridge University Press, 2014. (Cited on pages 2, 10, 14, 19, 21, 30, 31, 41, 57, 59, 60, 61, 71, 105, 107, $112,120,122,124,146$, and 147 , )

[90] D. Lucor, C.H. Su, and G.E. Karniadakis. "Generalized polynomial chaos and random oscillators". In: Int. J. Numer. Meth. Eng. 60.3 (2004), pp. 571-596. DOI: 10.1002/nme.976. (Cited on pages 1 and 83.) 
[91] D.J. Lunn, A. Thomas, N. Best, and D. Spiegelhalter. "Winbugs-a bayesian modelling framework: concepts, structure, and extensibility". In: Stat. Comput. 10.4 (2000), pp. 325-337. (Cited on pages 158 and 166.)

[92] G. Kallianpur. Stochastic Filtering Theory. New York: Springer, 1980. (Cited on page 141.)

[93] R.C. McKellar. "Development of a dynamic continuous-discretecontinuous model describing the lag phase of individual bacterial cells". In: J. Appl. Microbiol. 90.3 (2001), pp. 407-413. (Cited on page 150.)

[94] K. Mallick. "Random oscillator with general Gaussian noise". In: Physica A 384.1 (2007), pp. 64-68. DOI: 10.1016/j.physa.2007.04.070. (Cited on page 6 .)

[95] T.R. Malthus. An Essay on the Principal of Population. Oxford World's Classics Paperbacks. Oxford: Oxford University Press, 1999. (Cited on pages 87, 152, and 154.)

[96] Y.M. Marzouk, H.N. Najm, and L.A. Rahn. "Stochastic spectral methods for efficient Bayesian solution of inverse problems". In: J. Comput. Phys. 224.2 (2007), 560-586. DOI: 10.1016/j.jcp.2006.10.010. (Cited on pages 86 . 151 , and 158.)

[97] Y. Marzouk and D. Xiu. "A stochastic collocation approach to Bayesian inference in inverse problems". In: J. Commun. Comput. Phys. 6.4 (2009), pp. 826-847. DOI: 10.4208/cicp.2009.v6.p826. (Cited on pages 86, 151 . 158 , and 161 .)

[98] G. Maymon. Some Engineering Applications in Random Vibrations and Random Structures. American Institute of Aeronautics and Astronautics, 1998. (Cited on page 28, )

[99] M.E. Mincsovics. "Stability of one-step and linear multistep methods a matrix technique approach". In: Electron. J. Qual. Theo. 2016 (2016), pp. 1-10. DOI: 10.14232/ejqtde.2016.8.15. (Cited on page 140.)

[100] A. Mohammad-Djafari. "Bayesian inference for inverse problems". In: AIP Conference Proceedings. 617 (2002), pp. 477-496. (Cited on page 151.)

[101] J. Monod. "The growth of bacterial cultures". In: Annu. Rev. Microbiol. 3.1 (1949), pp. 371-394. (Cited on page 150.)

[102] J.D. Murray. Mathematical Biology I. Springer, 2002. (Cited on pages 40 87, 95, 150,152 , and 154.)

[103] National Spanish Health Survey (Encuesta Nacional de Salud de España, ENSE). http://pestadistico.inteligenciadegestion. msssi.es/publicoSNS/comun/ArbolNodos.aspx [accessed 28.085.18]. (Cited on pages xxii and 95.) 
[104] T. Neckel and F. Rupp. Random Differential Equations in Scientific Computing. München, Germany: Walter de Gruyter, 2013. (Cited on pages 1 and 40 .)

[105] A. Novick. "Growth of bacteria". In: Annu. Rev. Microbiol. 9.1 (1955), pp. 97-110. DOI: 10.1146/annurev.mi.09.100155.000525. (Cited on page 150.)

[106] B. Øksendal. Stochastic Differential Equations. An Introduction with Applications. Stochastic Modelling and Applied Probability 23. Heidelberg and New York: Springer-Verlag, 2003. (Cited on pages 57, 102, and 150.)

[107] J. Pitman. Probability. Springer, 1993. (Cited on page 112.)

[108] M. Plummer. "Jags: A program for analysis of bayesian graphical models using gibbs sampling". In: Proceedings of the 3rd international workshop on distributed statistical computing. Vol 124. Vienna, Austria, 2003. (Cited on page 158.)

[109] F. Pukelsheim. "The three sigma rule". In: Am. Stat. 48.2 (1994), pp. 8891. DOI: $10.2307 / 2684253$. (Cited on page 166.)

[110] L.v. Qiming and J.W. Pitchford. "Stochastic von Bertalanffy models with applications to fish recruitment". In: J. Theoret. Biol. 244 (2007), pp. 640-655. DOI: 10.1016/j.jtbi.2006.09.009. (Cited on page 57.)

[111] K. Renner-Martin, N. Brunner, M. Kühleitner, W.G. Nowak, and K. Scheicher. "On the exponent in the Von Bertalanffy growth model". In: PeerJ 6:e4205 (2018). DOI: 10.7717/peerj.4205. (Cited on page 56.)

[112] A.M. Roos. Modeling Population Dynamics. Netherlands: Notes from the University of Amsterdam, 2014. (Cited on pages 153 and 162.)

[113] W. Rudin. Principles of Mathematical Analysis. Ed. 3. Singapore: McGraw-Hill Education, 1976. (Cited on pages 17, 45, 74, and 107.)

[114] T.L. Saaty. Modern Nonlinear Equations. New York: Dover Publications Inc., 2003. ISBN: 9780486642321. (Cited on pages 57 and 58.)

[115] S. Salsa. Partial Differential Equations in Action, From Modelling to Theory. Universitext. Milan: Springer, 2010. (Cited on page 110.)

[116] A.A. Samarskii and P.N. Vabishchevich. Computational Heat Transfer. Mathematical Modelling. Wiley, 1996. (Cited on page 134.)

[117] F. Santonja and B.M. Chen-Charpentier. "Uncertainty quantification in simulations of epidemics using polynomial chaos". In: Comput. Math. Method. M. ID 742086 (2012), pp. 1-8. DOI: 10.1155/2012/742086. (Cited on pages $1,80,83$, and 86 .) 
[118] L.T. Santos, F.A. Dorini, and M.C.C. Cunha. "The probability density function to the random linear transport equation". In: Appl. Math. Comput. 216.5 (2010), pp. 1524-1530. DOI: 10.1016/j.amc.2010.03.001. (Cited on pages 6 and 135 .)

[119] D. Scott. "On optimal and data-based histograms". In: Biometrika 66.3 (1979), pp. 605-610. DOI:10.1093/biomet/66.3.605. (Cited on page 87.)

[120] M.A. Shah. "Stochastic logistic model for fish growth". In: Open J. Stat. 4 (2014), pp. 11-18. DOI: 10.4236/ojs.2014.41002. (Cited on page 57.)

[121] W. Shi and C. Zhang. "Error analysis of generalized polynomial chaos for nonlinear random ordinary differential equations". In: Appl. Numer. Math., 62.12 (2012), pp. 1954-1964. DOI: 10.1016/j.apnum.2012.08.007. (Cited on pages 1, 83, 85, and 161,)

[122] H. Slama, N. El-Bedwhey, A. El-Depsy, and M.M. Selim. "Solution of the finite Milne problem in stochastic media with RVT Technique". In: Eur. Phys. J. Plus 132 (2017), p. 505. DOI: 10.1140/epjp/i2017-11763-6. (Cited on page 135.)

[123] G.D. Smith. Numerical Solution of Partial Differential Equations: Finite Difference Methods. Ed. 3. New York: Oxford University Press, 1986. (Cited on page 141.)

[124] R.C. Smith. Uncertainty Quantification. Theory, Implementation and Applications. Philadelphia: SIAM, 2014. (Cited on pages 1, 6, and 102.)

[125] T.T. Soong. Random Differential Equations in Science and Engineering. New York: Academic Press, 1973. (Cited on pages 1, 2, 6, 7, 8, 12, 15 $23,27,28,40,41,44,45,46,57,60,82,102,103,107,108,134,136,137$. and 150 )

[126] D. Stanescu and B.M. Chen-Charpentier. "Random coefficient differential equation models for bacterial growth". In: Math. Comp. Model. 50.5-6 (2009), pp. 885-895. DOI: 10.1016/j.mcm.2009.05.017. (Cited on pages 1 . 83 , and 86 .)

[127] D. Stanescu, B.M. Chen-Charpentier, B.J. Jensen, and P.J. Colberg. "Random coefficient differential models of growth of anaerobic photosynthetic bacteria". In: Electron. T. Numer. Ana. 34 (2009), pp. 44-58. (Cited on pages xxii, xxiii, 86, 89, 90, 91, 151, 152, 154, 155, 156, 160, and 165.)

[128] M. Stokes, F. Chen, and F. Gunes. "An introduction to Bayesian analysis with SAS/STATR software". In: Proceedings of the SAS Global Forum 2014 Conference, SAS Institute Inc, Cary, USA (available at https://support.sas.com/resources/papers/proceedings14/SAS400-2014. pdf). Citeseer, 2014. (Cited on page 158.) 
[129] J.L. Strand. "Random Ordinary Differential Equations". In: J. Differ. Equations 7 (1970), pp. 538-553. DOI: 10.1016/0022-0396(70)90100-2. (Cited on pages 1, 8, 40, 57, 58, 82, 104, and 150.)

[130] B. Sudret. "Global sensitivity analysis using polynomial chaos expansions". In: Reliab. Eng. Syst. Safe. 93.7 (2008), pp. 964-979. DOI: 10.1016/j.ress.2008.10.008. (Cited on page 80.)

[131] A. Tarantola. Inverse Problem Theory and Methods for Model Parameter Estimation. Vol. 89. SIAM, 2005. (Cited on pages 158, 159 , and 166.)

[132] J.W. Thomas. Numerical Partial Differential Equations: Finite Difference Methods. Vol. 22. Springer Science \& Business Media, 2013. (Cited on page 137.)

[133] P. Turchin. "Does population ecology have general laws?" In: Oikos. 94.1 (2001), pp. 17-26. DOI: 10.1034/j.1600-0706.2001.11310.x. (Cited on page 152 .)

[134] M.M. Vainberg. Variational Methods for the Study of Nonlinear Operators. Holden-Day, 1964. (Cited on page 17.)

[135] A.W. van der Vaart. Asymptotic Statistics. Cambridge Series in Statistical and Probabilistic Mathematics. New York: Cambridge University Press, 2000. (Cited on pages 18, 46, 66, and 107.)

[136] P.F. Verhulst. "Notice sur la loi que la population suit dans son accroissement". In: Corr. Math. et Phys. 10 (1838), pp. 113-121. (Cited on pages 40,152 , and 154.)

[137] L. Villafuerte, C.A. Braumann, J.-C. Cortés, and L. Jódar. "Random differential operational calculus: theory and applications". In: Comput. Math. Appl. 59.1 (2010), pp. 115-125. DOI: 10.1016/j.camwa.2009.08.061. (Cited on pages 58, 59, 68, 107, and 108,)

[138] M. Villegas, F. Augustin, A. Gilg, A. Hmaidi, and U. Wever. "Application of the polynomial chaos expansion to the simulation of chemical reactors with uncertainties". In: Math. Comput. Simulat. 82.5 (2012), pp. 805817. DOI: 10.1016/j.matcom.2011.12.001. (Cited on pages 1 and 83.)

[139] C.R. White and R.S. Seymour. "Mammalian basal metabolic rate is proportional to body mass 2/3". In: Proceedings of the National Academy of Sciences of the United States of America 100 (2003), pp. 4046-4049. DOI: $10.1073 /$ pnas.0436428100. (Cited on page 56.)

[140] N. Wiener and A. Wintner. "Fourier-Stieltjes Transforms and Singular Infinite Convolutions". In: Am. J. Math. 60.3 (1938), pp. 513-522. DOI: 10.2307/2371591. (Cited on page 26.) 
[141] D. Williams. Probability with Martingales. Cambridge University Press, 1991. (Cited on page 113.)

[142] D. Xiu. Numerical Methods for Stochastic Computations. A Spectral Method Approach. Cambridge Texts in Applied Mathematics. New York: Princeton University Press, 2010. (Cited on pages 1, 82, 83, 87, 88, 150. $151,156,158$, and 160.)

[143] D. Xiu and G. Karniadakis. "The Wiener-Askey polynomial chaos for stochastic differential equations". In: J. Sci. Comput. 24 (2002), pp. 619 644. DOI: $10.1137 /$ S1064827501387826. (Cited on pages 1, 82, 83, 86, 87. 88,150 , and 160 .)

[144] D. Xiu and G. Karniadakis. "Modeling uncertainty in steady state diffusion problems via generalized polynomial chaos". In: Comput. Method. Appl. M. 191.43 (2002), pp. 4927-4948. DOI: 10.1016/S00457825(02)00421-8. (Cited on pages 1 and 83.)

[145] Z. Xu. "A stochastic analysis of steady and transient heat conduction in random media using a homogenization approach". In: Appl. Math. Model. 38.13 (2014), pp. 3233-3243. DOI: 10.1016/j.apm.2013.11.044. (Cited on page 102 .)

[146] Z. Xu, R. Tipireddy, and G. Lin. "Analytical approximation and numerical studies of one-dimensional elliptic equation with random coefficients". In: Appl. Math. Model. 40.9-10 (2016), pp. 5542-5559. DOI: 10.1016/j.apm.2015.12.041. (Cited on pages 2, 6, 80, 83, and 103.)

[147] A.C. Zaanen. Introduction to Operator Theory in Riesz Spaces. Springer, 1996. ISBN: 3540619895. (Cited on pages 65 and 106.)

[148] M.H. Zwietering, I. Jongenburger, F.M. Rombouts, and K. Van't Riet. "Modeling of the bacterial growth curve". In: Appl. Environ. Microb. 56.6 (1990), pp. 1875-1881. (Cited on page 150.) 